University of Louisville

ThinkIR: The University of Louisville's Institutional Repository

Electronic Theses and Dissertations

$12-2016$

\title{
Scalable production and applications of metal oxide nanowires.
}

Tu Quang Nguyen

University of Louisville

Follow this and additional works at: https://ir.library.louisville.edu/etd

Part of the Chemical Engineering Commons

\section{Recommended Citation}

Nguyen, Tu Quang, "Scalable production and applications of metal oxide nanowires." (2016). Electronic Theses and Dissertations. Paper 2562.

https://doi.org/10.18297/etd/2562

This Doctoral Dissertation is brought to you for free and open access by ThinkIR: The University of Louisville's Institutional Repository. It has been accepted for inclusion in Electronic Theses and Dissertations by an authorized administrator of ThinkIR: The University of Louisville's Institutional Repository. This title appears here courtesy of the author, who has retained all other copyrights. For more information, please contact thinkir@louisville.edu. 


\title{
SCALABLE PRODUCTION AND APPLICATIONS OF METAL OXIDE NANOWIRES
}

\author{
By \\ Tu Quang Nguyen \\ B.E., Hanoi University of Science and Technology, 2007 \\ M.S., Kongju National University, 2010 \\ A Dissertation \\ Submitted to the Faculty of \\ The J. B. Speed School of Engineering of the University of Louisville \\ In Partial Fulfillment of the Requirements \\ for the Degree of \\ Doctor of Philosophy in Chemical Engineering \\ Department of Chemical Engineering \\ University of Louisville \\ Louisville, KY 40292
}

December 2016 
CCopyright 2016 by Tu Quang Nguyen

All rights reserve 



\title{
SCALABLE PRODUCTION AND APPLICATIONS OF METAL OXIDE NANOWIRES
}

\author{
By \\ Tu Quang Nguyen \\ B.Eng., Hanoi University of Science and Technology, 2007 \\ M.S., Kongju National University, 2010
}

A Dissertation Approved on

August $5^{\text {th }} 2016$

By the Following Dissertation Committee

Dr. Mahendra K. Sunkara (Dissertation Director)

Dr. Thomas L. Starr

Dr. R. Eric Berson

Dr. Gamini U. Sumanasekera

Dr. Theodore L. Druffel 


\section{DEDICATION}

This dissertation is dedicated to

my parents Nguyễn Quang Tư and Nguyễn Thị Định, sister Nguyễn Thị Hiền, brother Nguyễn Quang Tuân, beloved wife Uyen Duong, and my lovely daughters Sophia Nguyen and Ruby Nguyen. 


\section{ACKNOWLEDGEMENTS}

First, I would like to express my deepest gratitude to my graduate advisor, Dr. Mahendra K. Sunkara, for giving me an opportunity to be a part of his wonderful research group at the University of Louisville. He was the reason I made the decision to move from Florida to Louisville. I extremely appreciate his constant support and endless encouragement throughout my Ph.D. He is the best mentor I have ever known. His compassion and friendly behavior have made an enjoyable environment for all his students to study and learn.

I would like to acknowledge Dr. Thomas Starr, Dr. R. Eric Berson, Dr. Gamini Sumanasekera and Dr. Theodore Druffel for serving as my committee members. I appreciate their feedback and suggestions that have greatly improved this dissertation.

I would like to thank Vasanthi Sunkara, the CEO \& President of Advanced Energy Materials, LLC (AdEM) for giving me an opportunity to finish up my research work at the company. I also acknowledge Luke Guhy, Juan He, Siva Vasireddy, and Vivek Kumar for help with my work during last two years at AdEM. I will never forget the feeling of accomplishment when we setup the lab and reactor on our own.

I would like to thank all my lab members Venkat Vendra, Ben Russell, Daniel Jaramillo, Dustin Cummins, Swathi Sunkara, Alejandro Martinez, Apolo Nambo, Veerendra Atla and Dr. Arjun Thapa for helping me with my work and making my Ph.D. 
life enjoyable. Special thanks to Dr. Jacek Jasinski for all of his help with material characterization.

I am thankful to Patty Lumley and Eunice Salazar for their patience and help with registration and related paperwork.

I would also like to thank the Conn Center for Renewable Energy Research for facilities and access to characterization equipment, as well as the Department of Chemical Engineering and University of Louisville for giving the opportunity to finish up my Ph.D. I am also thankful to the funding support from Grosscurth Fellowship, US Department of Energy, and National Foundation Science.

I am very grateful to my parents for their endless love and encouragement throughout my life. My father who never stopped supporting me since I was a kid. Lastly, I would like to thank my wife Uyen Duong, for her patience, encouragement and endless love for making this dissertation a success. I also owe a great deal to my kids Sophia Nguyen and Ruby Nguyen for being with me during tough years of my Ph.D.

Tu Quang Nguyen

September 2016 


\begin{abstract}
SCALABLE PRODUCTION AND APPLICATIONS OF METAL OXIDE NANOWIRES

Tu Quang Nguyen

August $5^{\text {th }} 2016$
\end{abstract}

Metal oxide nanowires are materials of interest in number of applications such as lithium ion batteries, solar cells, catalyst support, and gas sensing due to their unique charge transport properties and short diffusion length scales. To incorporate nanowires for any applications, one would need hundreds of grams to kilograms of these nanowires. However, state-of-the-art methods for producing metal oxide nanowires are limited to producing only milligrams to a gram in a batch. Hence, there is a need to develop scalable and cost effective processes and reactors to address this challenge.

Direct gas phase oxidation of zinc metal powders using a downward atmospheric microwave plasma allowed producing 50-100 grams of zinc oxide nanowires per day. The downstream plasma reactor has certain limitations in terms of scalability: short residence time and plasma instability. Thus, a more improved reactor design is needed for continuous production of nanowire materials at commercially viable production rates.

In this dissertation, a fluidized bed reactor is designed and studied for scalable production. The key feature of the reactor involves feed particles being fluidized in the 
flame to increase residence time. The reactor is equipped with cyclone and bag house filter housing enabling efficient powder collection and allows continuous production. Experiments using fluidized bed reactor produced single crystalline nanowires of about 30-200 $\mathrm{nm}$ in diameter and $0.5-2 \mu \mathrm{m}$ in length. The nanowire morphology could be controlled by gas flow rate, powder feeding rate and flame type. A production rate of 1.2 $\mathrm{kg}$ of nanowires per hour and yield of about $90 \%$ has been demonstrated. The zinc oxide nanowires prepared in this work have been tested as catalyst support for hydrodesulfurization of diesel. The sulfur content was reduced from $200 \mathrm{ppm}$ to less than 1 ppm and the catalyst was active for over 100 hours.

Another concept termed as solvo-plasma oxidation has been demonstrated earlier with producing nanowires of titania and related transition metals. The technique involves the synthesis of nanowires using oxidation of metal containing precursors in the presence of alkali salts. The reaction time scales were on the order of few seconds to a minute which is about 3 to 4 orders of magnitude faster than that using a hydrothermal method. Growth rates higher than $1 \mu \mathrm{m} / \mathrm{min}$ were obtained. Here, the concept is studied with tin oxide first to see the ability to produce nanowires and then to understand the mechanism responsible for one-dimensional growth. Experiments reveal that the intermediate phase of potassium stannate could be held responsible for the 1D growth. In addition, experiments also confirm that the solvo-plasma technique is generic for synthesizing most of metal oxides nanowires including titania, cobalt oxide, manganese oxide, tungsten oxide, zinc oxide, and tin oxide nanowires. A simple lab-scale roll-to-roll setup can produce up to 300 grams per hour. 
Tin oxide nanowires find applications in lithium ion batteries. However, asproduced tin oxide nanowire powders are not chemically stable with respect to cycling. Previously, the nanowires were shown to be stable when they were reduced to produce decoration of tin clusters on their surfaces. Here, the tin oxide nanowires were treated with ultra-thin layers of titania or alumina coating as thin as $1 \mathrm{~nm}$ to understand the stability with respect to lithium ion cycling. No initial capacity loss due to SEI formation was found which increased the reversible capacity retention. Both titania- and aluminacoated tin oxide nanowires exhibited tin migration through the coatings to form tin nanoclusters. The compressive stress build-up during lithium intercalation and the enhanced diffusion of tin during lithium de-intercalation allowed for migration of tin to outside of coatings. The results obtained with tin should be applicable to other high capacity materials such as silicon.

In summary, two types of scalable production for metal oxide nanowires were studied: A fluidized bed involving plasma and other types of flames for implementing direct oxidation of low-melting metals is studied with great success. In the second concept, the plasma oxidation of tin oxide materials dissolved in alkali salts is studied to understand the intermediate steps responsible for one-dimensional growth. Studies further showed that the second concept could also be implemented using fluidized bed reactor for scalable production. Finally, the bulk produced zinc oxide nanowires and tin oxide nanowires have been tested in hydro-desulfurization and lithium ion battery applications, respectively. In the case of lithium ion battery application, the bulk produced nanowires exhibited stability with cycling when coated with ultra-thin layers of tinania and alumina. 


\section{TABLE OF CONTENTS}

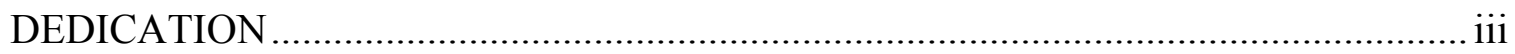

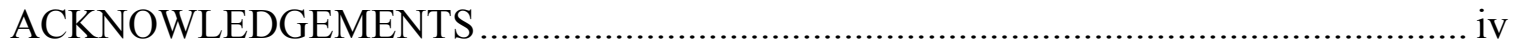

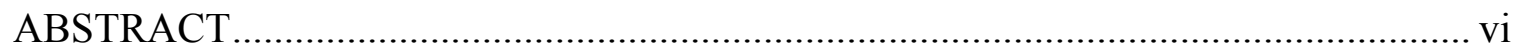

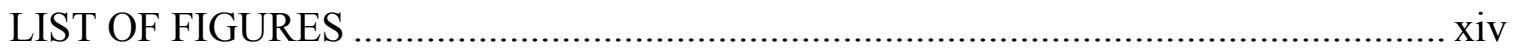

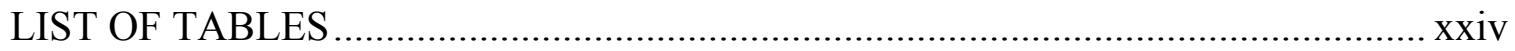

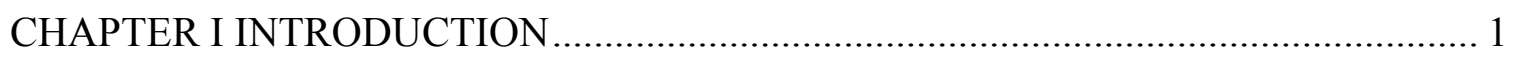

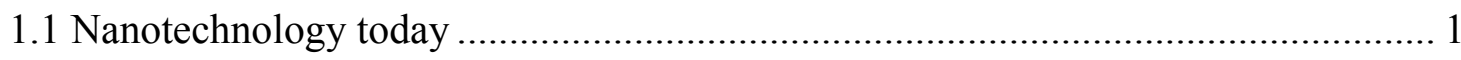

1.2 Applications of metal oxide nanowires....................................................... 2

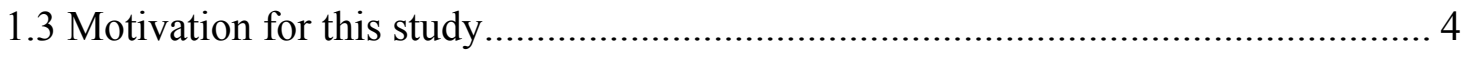

1.4 Proposed concepts for scalable production and uses for metal oxide nanowires ..... 6

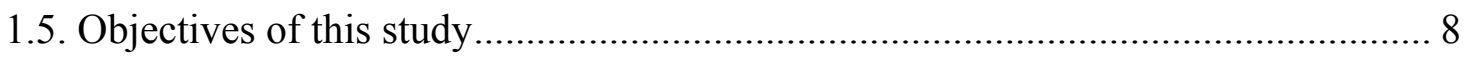

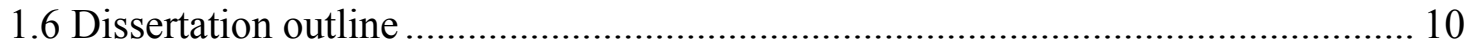

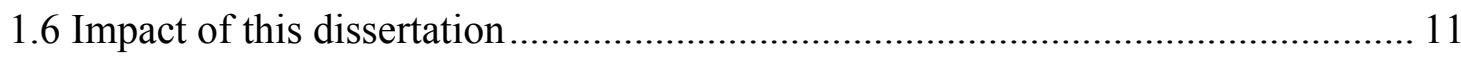

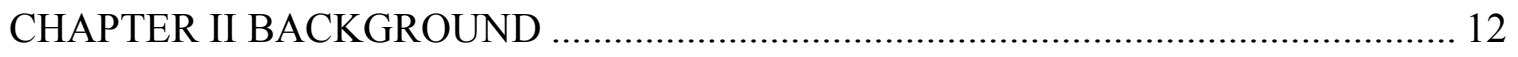

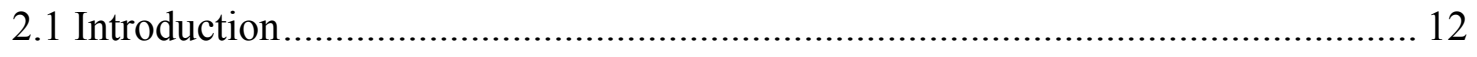

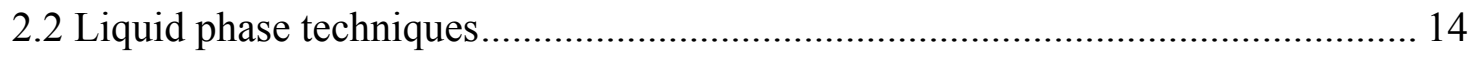

2.2.2 Hydrothermal and solvothermal methods ............................................ 15 
2.2.3 Electrochemical and sol-gel syntheses........................................................ 17

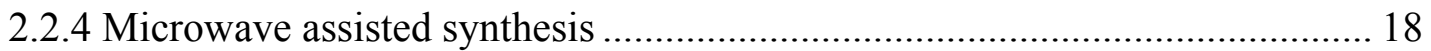

2.2.5 Summary of liquid phase techniques ........................................................... 19

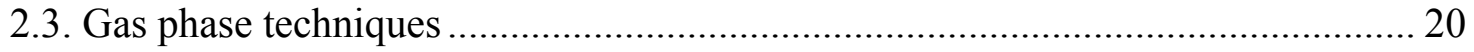

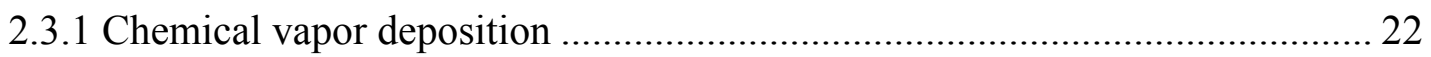

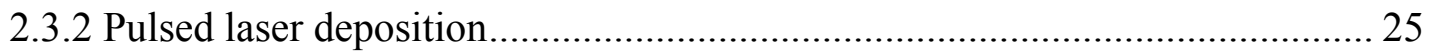

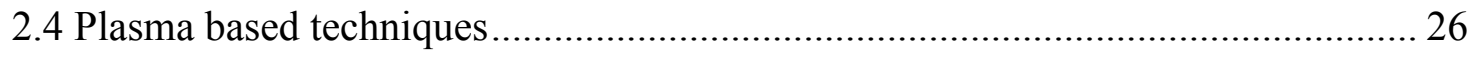

2.4.1 Plasma-enhanced chemical vapor deposition ................................................ 29

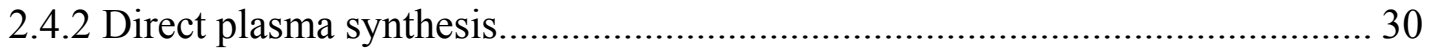

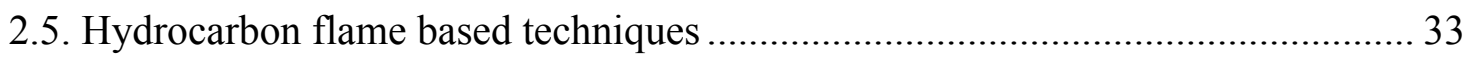

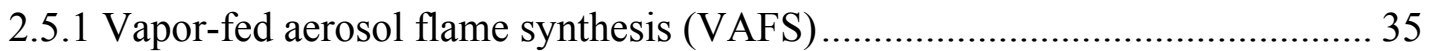

2.5.2 Flame-assisted spray pyrolysis (FASP) ………………….......................... 35

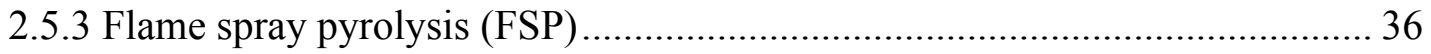

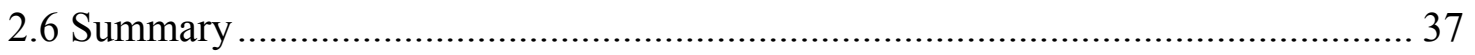

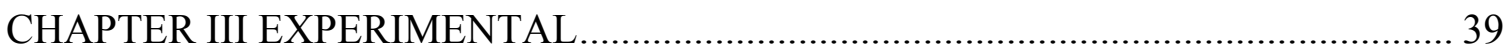

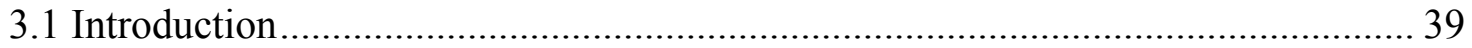

3.2 The downstream atmospheric microwave plasma reactor ...................................... 39

3.2.1 Challenges with the downstream atmospheric microwave plasma reactor ..... 41

3.2.2 Reactor design modification and results ......................................................... 43

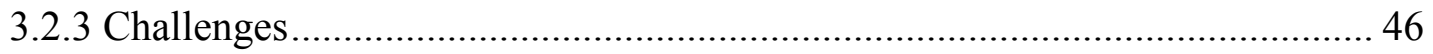

3.3 Design of a fluidized bed reactor for mass production of metal oxide nanowires . 49

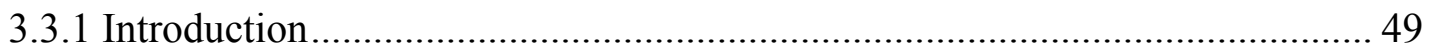

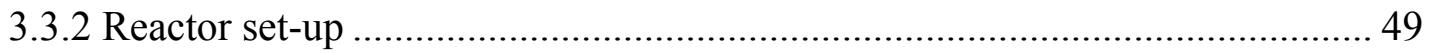


3.3.3 Reactor material, shape and dimensions ............................................. 54

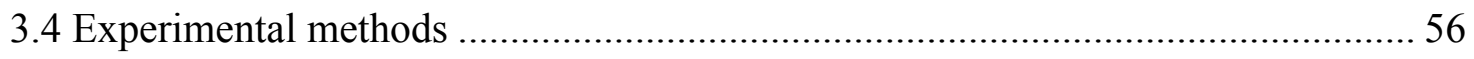

3.4.1 Synthesis of zinc oxide using fluidized bed reactor..................................... 56

3.4.2 Catalyst preparation and testing for zinc oxide nanowires .......................... 57

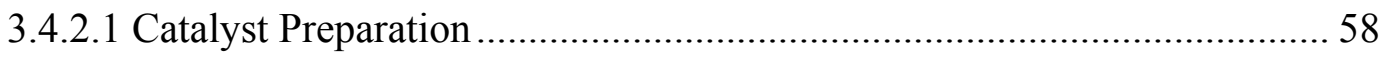

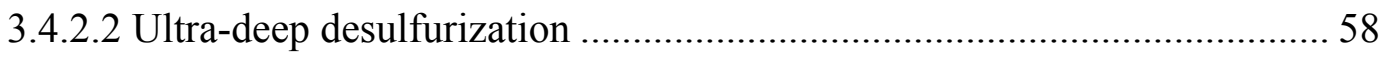

3.4.3 Synthesis of metal oxide using upstream plasma reactor via solvo plasma .... 60

3.4.4 Detail of computational fluid mechanics simulations............................... 61

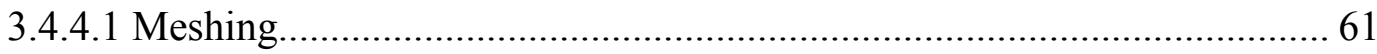

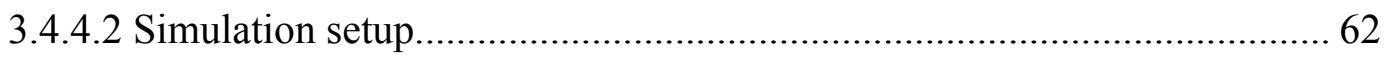

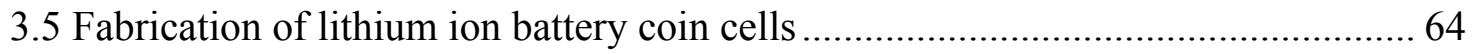

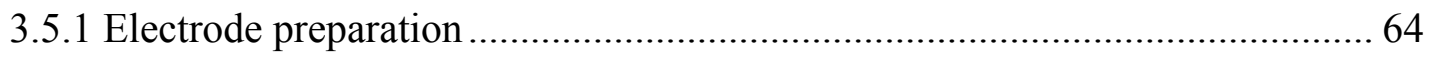

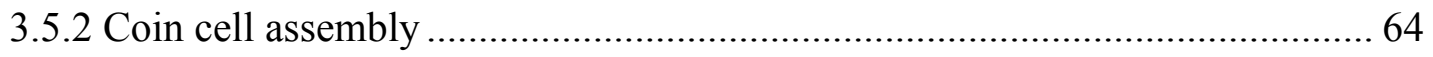

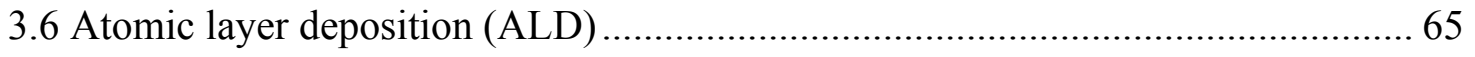

3.7 Materials characterization: Electron Microscopy and Diffraction Techniques ...... 66

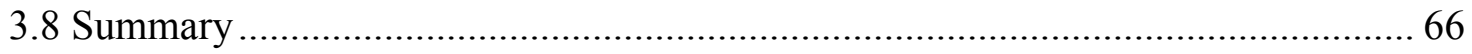

\section{CHAPTER IV MASS PRODUCTION STUDIES OF ZINC OXIDE NANOWIRES}

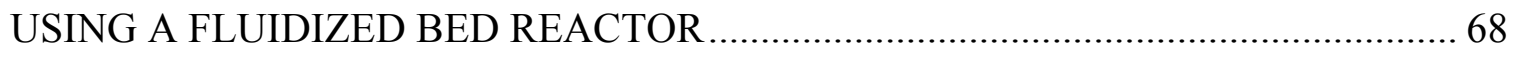

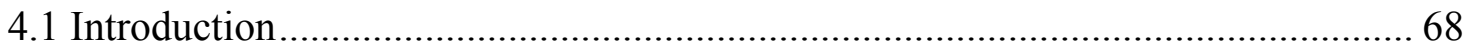

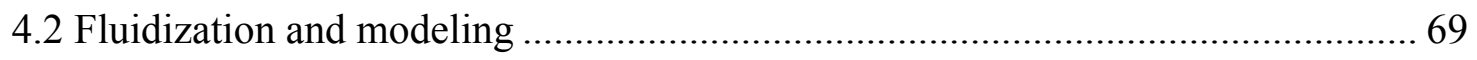

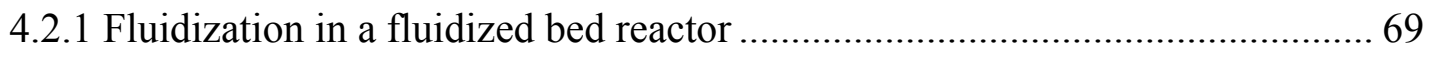

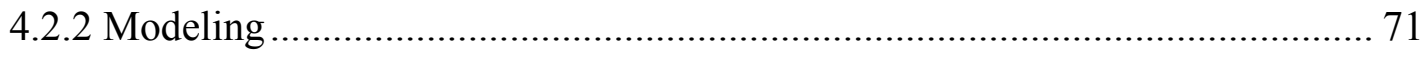

4.3 Production of zinc oxide nanowires using atmospheric microwave plasma ......... 76 
4.4 Production of zinc oxide nanowires using hydrocarbon flame

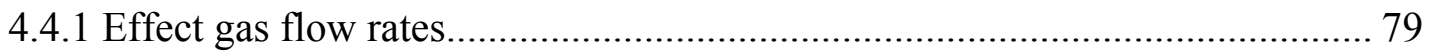

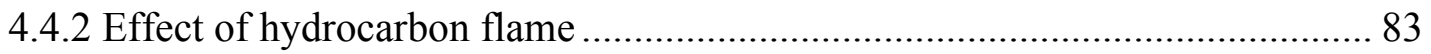

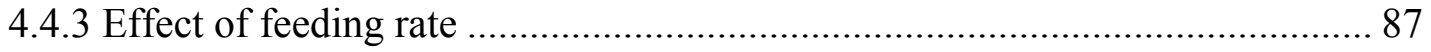

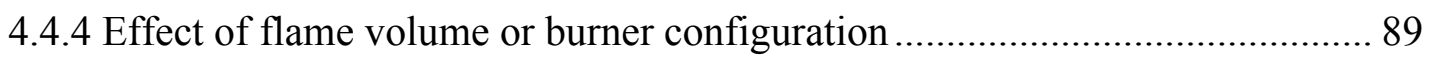

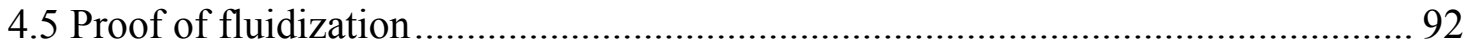

4.6 Zinc oxide as catalyst support for hydro-desulfurization of diesel....................... 94

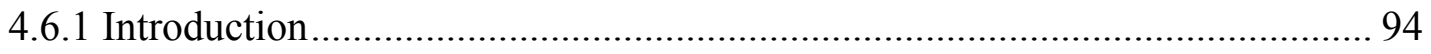

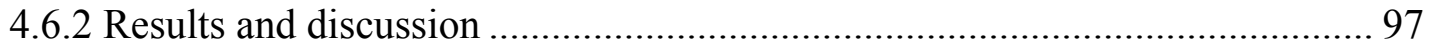

4.6.2.1 Textural Properties of Catalysts ....................................................... 97

4.6.2.2 Ultra-deep Desulfurization Activity Testing of Catalysts ...................... 99

4.6.2.3 Discussion on Activity and Mechanism .......................................... 101

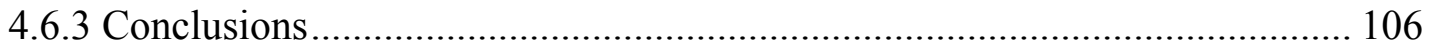

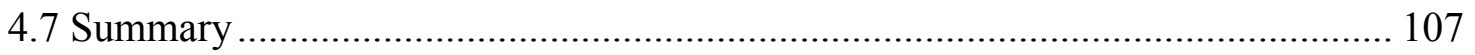

\section{CHAPTER V SCALABLE SOLVO-PLASMA PRODUCTION OF TIN OXIDE}

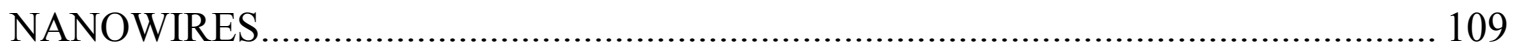

5.1 Scalable solvo plasma production of tin oxide nanowires ................................ 109

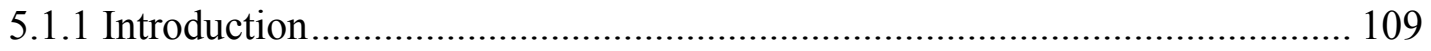

5.1.2 Results and Discussion ......................................................................... 111

5.2 Growth mechanism for stannate nanowires during solvo-plasma oxidation ........ 122

5.3 Porous tin oxide as anodes for lithium ion battery application.......................... 128

5.4 Generic solvo plasma production of metal oxide nanowires ............................ 130 
5.5 Scalability of solvo-plasma technique using conveyor belt and fluidized bed reactor

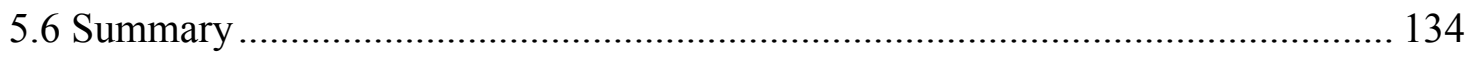

CHAPTER VI PROTECTIVE COATING IN NANOWIRE BASED ANODES ......... 136

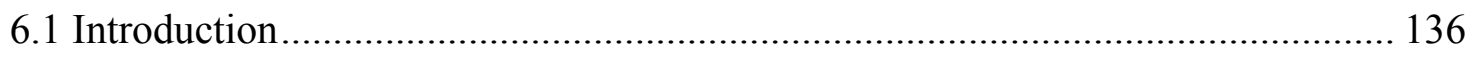

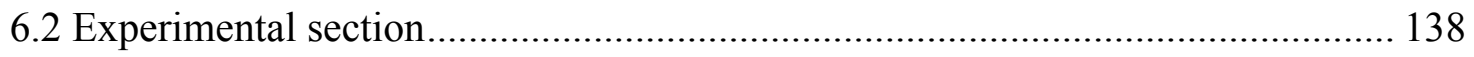

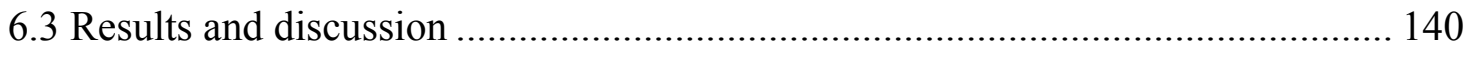

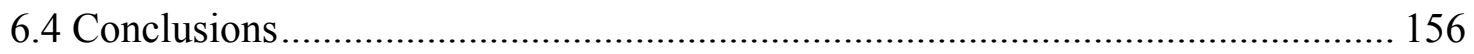

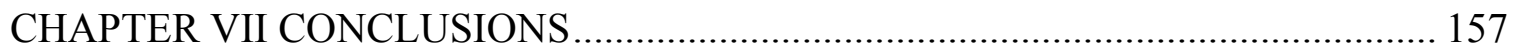

7.1 The fluidized bed reactor ........................................................................ 157

7.2 Large scale production of zinc oxide nanowires using fluidized bed reactor....... 158

7.3 Solvo-plasma production of metal oxide nanowires .................................... 158

7.4 Applications of nanowires as catalyst supports and lithium ion batteries ........... 159

CHAPTER VIII RECOMMENDATIONS FOR FUTURE WORK ........................... 161

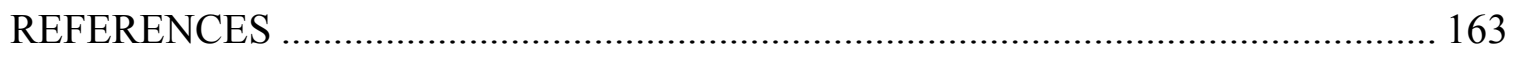

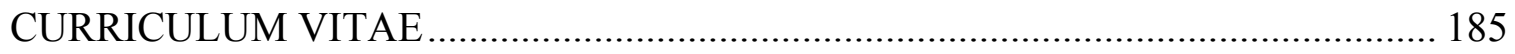




\section{LIST OF FIGURES}

Figure 1.2.1: Schematic of electron transport through a) nanoparticles and b) nanowires

Figure 1.2.2 Schematic of sinterability of a) conventional nanoparticles and b) nanowire powders

Figure 1.4.1: a) Schematic of direct oxidation of metal powder and b) proposed reactor setup

Figure 1.4.2: Schematic of solvo-plasma oxidation of titania nanowire arrays and powder

Figure 2.1.1: Schematic formation of titania nanoparticles in gas phase using TTIP precursor

Figure 2.3.1: Schematic of 1D growth using VLS: (a) metal catalyst on substrate, (b) formation of liquid alloy droplets, (c) and (d) supersaturation leads to 1D growth by tipled growth

Figure 2.3.2: Schematic of a CVD reactor

Figure 2.3.3: Schematic of hotwire CVD reactor

Figure 2.3.4: Schematic of reactor for pulsed laser deposition

Figure 2.4.1: Schematic of inductive coupled plasma and capacitive coupled plasma

Figure 2.4.2: Schematic of atmospheric microwave plasma in Sunkara's group 
Figure 2.4.3: Schematic of PECVD without using catalyst (a) and with using catalyst (b)

Figure 2.4.4: Schematic of direct plasma synthesis: (c) plasma flight-through and (d) direct contact plasma synthesis

Figure 2.4.5: Nanowire production rate for zinc oxide nanowires by different plasma based technique

Figure 2.5.1: Two possible mechanism for particle formation in flame synthesis

Figure 2.5.2: Time scale requires for different synthesis techniques. Direct plasma synthesis offer shortest time scale compared to all other methods

Figure 3.2.1: Schematic of the downstream atmospheric microwave plasma system

Figure 3.2.2: Schematic of the Downstream Atmospheric Microwave Plasma Reactor

Figure 3.2.3: Schematic of the semi-automatic powder feeder

Figure 3.2.4: Schematic of the modified reactor and photos of plasma column, filter, cooling coil

Figure 3.2.5: SEM image of $\mathrm{ZnO}$ nanowires (top left), TEM of $\mathrm{ZnO}$ nanowires (top right), Bottles of $\mathrm{ZnO}$ nanowire powder, $\mathrm{XRD}$ pattern of of $\mathrm{ZnO}$ nanowire power collected in filter housing.

Figure 3.2.6: SEM image of powder collected in the Filter at 5 grams per minute; a photo showing deposition on quartz tube and schematic of the downstream atmospheric microwave plasma reactor

Figure 3.3.1: Schematic of fluidized bed reactor system with an atmospheric microwave plasma system 
Figure 3.3.2: Schematic of fluidized bed reactor body

Figure 3.3.3: A photo showing plasma flame inside reactor chamber

Figure 3.3.4: Schematic drawing of hydrocarbon burner

Figure 3.3.5: Velocity gradient along the reactor created by conical shape of reactor

Figure 3.3.6: Contours of velocity magnitude of different reactor shapes: a) conical shape, b) cylindrical shape, and c) very-wide-top conical shape

Figure 3.4.1: Extrudates of nickel acetate- $\mathrm{ZnO}-\gamma-\mathrm{Al}_{2} \mathrm{O}_{3}$ and $\mathrm{NiO}-\mathrm{ZnO}-\gamma-\mathrm{Al}_{2} \mathrm{O}_{3}$

Figure 3.4.2: (a) Photograph of the fixed bed reactor used for desulfurization experiments; (b) Schematic of three zone packing design used in our packed column.

Figure 3.4.3: Meshing size and the reactor geometry after the mesh generation

Figure 3.5.1: Schematic of lithium ion coin cell assembly

Figure 4.2.1: Contours of velocity magnitude at different flow inlet velocities of a) $1 \mathrm{~ms}^{-}$

, a) $10 \mathrm{~ms}^{-1}$, and a) $40 \mathrm{~ms}^{-1}$

Figure 4.2.2: Streamlines: Colored by velocity magnitudes

Figure 4.2.3: Particle tracking: Colored by particle size

Figure 4.3.1: SEM image of zinc oxide nanowires collected under the filter a) and under cyclone b) (Plasma power: $1.5 \mathrm{~kW}$. Air: $20 \mathrm{LPM}+1 \mathrm{CFM}+5 \mathrm{CFM})$

Figure 4.3.2: Aspect ratio, diameter and length distributions of zinc oxide produced using plasma

Figure 4.4.1: SEM image of zinc metal particles as starting materials a), SEM image of 
typical resulting zinc oxide nanowires b); TEM image of typical resulting zinc oxide nanowires c); HR-TEM image of typical resulting zinc oxide nanowires d)

Figure 4.4.2: XRD pattern of resulting zinc oxide nanowire powder

Figure 4.4.3: SEM images of zinc oxide nanowires produced using different oxygen flow rates: a) $15 \mathrm{LPM}$, b) $30 \mathrm{LPM}$, and c) $45 \mathrm{LPM}$

Figure 4.4.4: Effect of oxygen flow rate on nanowire morphology: first row of 15 LPM, second row of 30 LPM, and third row of 45 LPM

Figure 4.4.5: SEM images of zinc oxide nanowires produced using different propane flow rates: a) 2 LPM, b) 5 LPM, and c) 10 LPM

Figure 4.4.6: Effect of propane flow rate on nanowire morphology: first row of 2 LPM, second row of $5 \mathrm{LPM}$ gram $/ \mathrm{min}$, and third row of $10 \mathrm{LPM}$

Figure 4.4.7: SEM images of zinc oxide nanowires produced using different hydrocarbons: a) methane, b) propane, and c) acetylene

Figure 4.4.8: Effect of hydrocarbon type on nanowire morphology: first row of methane, second row of propane, and third row of acetylene

Figure 4.4.9: Effect of hydrocarbon type or heat of combustion on nanowire length and diameter

Figure 4.4.10: SEM images of zinc oxide nanowires produced using different feeding rates: a) 3 grams $/ \mathrm{min}$, b) 12 grams $/ \mathrm{min}$, and c) 24 grams $/ \mathrm{min}$

Figure 4.4.11: Effect of feeding rate on nanowire morphology: first row of 3 grams $/ \mathrm{min}$, second row of 12 grams $/ \mathrm{min}$, and third row of $24 \mathrm{grams} / \mathrm{min}$ 
Figure 4.4.12: Schematic of burner configuration and corresponding flame length

Figure 4.4.13: Effect of flame length on production rate and conversion

Figure 4.4.14: A photo of $30 \mathrm{~kg}$ of zinc nanowire powder produced using fluidized bed reactor

Figure 4.5.1: Schematic of the fluidized bed reactor with the metal mesh at the middle portion. The corresponding SEM images of zinc oxide powder collected on upside and downside of the metal mesh

Figure 4.7.1: A schematic showing uniform morphologies for nanoclusters produced on mono-crystalline facets of c-plane oriented $\mathrm{ZnO}$ nanowire.

Figure 4.7.2: Sulfur content in diesel samples at different times-on-stream: Comparison of catalysts using different Ni loading amounts on $\mathrm{ZnO} \mathrm{NW}$

Figure 4.7.3: Color of diesel samples with time

Figure 4.7.4: SEM micrographs and XRD patterns of fresh and spent catalyst $15 \% \mathrm{Ni}$, $58.7 \% \mathrm{ZnO}, 26.3 \% \gamma-\mathrm{A} 12 \mathrm{O} 3$

Figure 4.7.5: XRD patterns for fresh and spent catalysts with compositions (Left) 12\% $\mathrm{Ni}, 58.7 \% \mathrm{ZnO}, 29.3 \% \mathrm{y}-\mathrm{Al}_{2} \mathrm{O}_{3}$; and (Right) $12 \% \mathrm{Ni}, 3 \% \mathrm{Mo}, 58.7 \% \mathrm{ZnO}, 26.3 \% \mathrm{y}-$ $\mathrm{A} 12 \mathrm{O} 3$

Figure 4.7.6: TEM micrographs of $15 \% \mathrm{Ni}, 58.7 \% \mathrm{ZnO}, 26.3 \% \gamma-\mathrm{Al} 2 \mathrm{O} 3$ (a) fresh catalyst; (b) spent catalyst. Images do not show the same nanowire but different ones from the same sample. 
Figure 4.7.7: TEM micrographs of spent catalyst $\left(12 \% \mathrm{Ni}, 58.7 \% \mathrm{ZnO}, 29.3 \% \gamma-\mathrm{Al}_{2} \mathrm{O}_{3}\right)$ : (A) Low-magnification bright filed TEM micrograph; (B) HRTEM image of the selected area in catalyst (A): showing the $\mathrm{Ni}$ and $\mathrm{NiO}$ cluster; (C) HRTEM image of the selected area in catalyst (A): showing the tail area of the catalyst; (D) HRTEM image of the selected area in catalyst (A): showing the center area of $\mathrm{ZnO} \mathrm{NW}$.

Figure 4.7.8: Proposed reaction mechanism for desulfurization on $\mathrm{Ni} / \mathrm{ZnO}$ nanowires

Figure 5.1.1: Photograph of an actual experiment: plasma exposure of tin oxide precursor and $\mathrm{KOH}$ paste on quartz substrate a), a bottle of tin oxide nanowire powder of 10 grams produced in one hour reaction time

Figure 5.1.2: Scanning electron microscopy of tin oxide nanowire powder after annealing a), TEM images b) and c) showing the porous structure and twining effect, (inset) SAED of tin oxide nanowires, HR-TEM image showing the matching interplanar lattice spacing of (110) and (101) plane of tin oxide nanowires d).

Figure 5.1.3: Powder XRD pattern of samples after annealing in plasma showing the pure phase of rutile tin oxide while the as-synthesized samples showing additional intermediate compounds.

Figure 5.1.4: HR-TEM images of as-synthesized samples showing well-matched interplanar lattice spacing of rutile tin oxide in (a) and (b), $\mathrm{K}_{2} \mathrm{SnO}_{3} \cdot \mathrm{H}_{2} \mathrm{O}$ in (c) and (d)

Figure 5.1.5: Raman spectrum of tin oxide after plasma annealing in comparison with bulk tin oxide 
Figure 5.1.6: SEM images of (a) tin oxide precursor and as-synthesized $\mathrm{K}_{2} \mathrm{SnO}_{3}$ nanowires at weight ratios of $\mathrm{SnO}_{2}$ to $\mathrm{KOH}$ of (b) $3: 1$, (c) 1:1, (d) 1:3. Experiments were conducting using plasma flame with power of $1.2 \mathrm{~kW}$ at a duration of 60 seconds

Figure 5.1.7: SEM image of as-synthesized samples using a) $\mathrm{SnO}_{2}+\mathrm{KCl}$, b) $\mathrm{Sn}+\mathrm{KOH}$, c) $\mathrm{Sn}+\mathrm{KCl}$, d) $\mathrm{SnO}+\mathrm{KOH}$, e) $\mathrm{K}_{2} \mathrm{SnO}_{3}$, f) $\mathrm{SnO}_{2}+\mathrm{KOH}$. Sample a) to e) prepared using plasma heating for 60 seconds, sample f) prepared using thermal heating at $900^{\circ} \mathrm{C}$ for 2 hrs.

Figure 5.1.8: SEM image of as-synthesized sample prepared at different distance away from the outlet of applicator: a) 1 inch, b) two inches, and c) 3 inches. The bottom row showing the actual plasma flame with red marks as position of samples

Figure 5.1.9: SEM image of as-synthesized sample prepared at different time scale using large tin oxide particles as precursor: a) 20 seconds, b) 60 seconds, and c) 5 minutes. Lower row showing images at higher magnification

Figure 5.2.1: Schematic showing the proposed growth mechanism for growth of tin oxide nanowires. a) Mixture of tin oxide particle and $\mathrm{KOH}$ in plasma flame, b) $\mathrm{KOH}$ got melted into free moving $\mathrm{K}^{+}$and $\mathrm{OH}^{-}$ions, c) diffusion of oxygen radicals, $\mathrm{K}^{+}$and $\mathrm{OH}^{-}$ions into tin oxide particle to form $\mathrm{K}_{2} \mathrm{SnO}_{3}$, d) $\mathrm{K}_{2} \mathrm{SnO}_{3}$ nucleus forming under supersaturation of $\mathrm{K}_{2} \mathrm{SnO}_{3}$, e) layered structure $\mathrm{K}_{2} \mathrm{SnO}_{3}$ grown due to active edge.

Figure 5.2.2: In-situ measurement of surface temperature of samples on quartz substrate

Figure 5.2.3: XRD pattern of a) $\mathrm{K}_{2} \mathrm{SnO}_{3}$ particle precursor, b) as-synthesized nanowires using $\mathrm{K}_{2} \mathrm{SnO}_{3}$ particles, c) as-synthesized nanowires using tin mono oxide particles, d) tin mono oxide particle precursor, e) as-synthesized nanowires using tin oxide particles for 
10 seconds exposure time, f) as-synthesized nanowires using tin oxide particles for 60 seconds exposure time, g) $\mathrm{SnO}_{2}$ particle precursor

Figure 5.3.1. Galvanostatic curves a) and discharge capacity vs. cycle number b) of tin nanoclusters covered tin oxide nanowires at current density of $100 \mathrm{~mA} / \mathrm{g}$

Figure 5.4.1: SEM image a), TEM image b), XRD pattern c) and Raman spectrum c) of resulting zinc oxide nanowires prepared using solvo plasma oxidation

Figure 5.5.1: a) Roll-to-roll setup for commercial production of tin oxide nanowire powder; b) A photo of lab-scale conveyor belt

Figure 5.5.2: SEM images of as-synthesized nanowires produced using the downstream atmospheric microwave plasma reactor. a) titanate, b) stannate, and c) stannate nanowires produced using $\mathrm{K}_{2} \mathrm{O}$. $\mathrm{TiO}_{2}$ powder, $\mathrm{K}_{2} \mathrm{SnO}_{3}$ powder and mixture of $\mathrm{SnO}_{2}$ particles and $\mathrm{KOH}$, respectively.

Figure 6.1 SEM and TEM (inset) images of titania-coated SnO2 NWs before cycling

Figure 6.2 a) Cyclic voltammetry of titania-coated $\mathrm{SnO} 2 \mathrm{NWs}$ at the voltage range of 2.2$0.005 \mathrm{~V}$ using scan speed of $5 \mathrm{mV} / \mathrm{min}$. b) Charge-discharge capacity of pure $\mathrm{SnO} 2$ NWs and titania-coated $\mathrm{SnO} 2 \mathrm{NWs}$ at the voltage range of 2.2-0.005 V using current density of $60 \mathrm{~mA} / \mathrm{g}$; c) Comparison of capacity vs. cycle number of SnO2 NWs and titania-coated $\mathrm{SnO} 2 \mathrm{NWs}$ at currents of 60,700 , and $1500 \mathrm{~mA} / \mathrm{g}$; d) Comparison of capacity vs. cycle number of $\mathrm{SnO} 2 \mathrm{NWs}$ and alumina-coated $\mathrm{SnO} 2 \mathrm{NWs}$ at currents of 60,700 , and $1500 \mathrm{~mA} / \mathrm{g}$

Figure 6.3: Cyclic voltammetry of $\mathrm{SnO}_{2} \mathrm{NWs}$ at the voltage range of $2.2 \div 0.005 \mathrm{~V}$ using scan speed of $5 \mathrm{mV} / \mathrm{min}$ 
Figure 6.4: The charge-discharge capacities with times vs. voltage profiles of titaniacoated $\mathrm{SnO}_{2} \mathrm{NWs}$ electrode at 3.0-1.0 V

Figure 6.5: Energy band diagram of $\mathrm{SnO}_{2}$ and $\mathrm{Al}_{2} \mathrm{O}_{3}$ (a), $\mathrm{TiO}_{2}$ (b) with respect to the electrochemical scale. Ev, Ec, and Eg represent valence band maxima, conduction band minima and bandgap, respectively

Figure 6.6: The XRD pattern showing no peak of titania due to the amorphous phase, the tin oxides were completely reduced to tin after cycling (a), SEM and TEM (inset) images of titania-coated $\mathrm{SnO} 2 \mathrm{NWs}$ after 100 cycles showing the original morphology (b)

Figure 6.7: (a) Charge and discharge capacities vs. cycle number of titania-coated $\mathrm{SnO}_{2}$ NWs at current density of $1500 \mathrm{~mA} / \mathrm{g}$; (b) the photograph of delaminated electrode: the tin oxide film (black in color) was completely delaminated from stainless substrate and adhered onto the separator (white in color).

Figure 6.8: EDS data of $15 \mathrm{~nm}$ titania-coated $\mathrm{SnO}_{2} \mathrm{NWs}$ after cycling

Figure 6.9: EDS data of titania-coated $\mathrm{SnO}_{2} \mathrm{NWs}$ after cycling at votage of $1.0-3.0 \mathrm{~V}$

Figure 6.10: HR-TEM images of tin nanoclusters evolution on the surface of titania coating layer: tin started squeezing out a) and b); coalescing to form tin nanocluster c); tin nanoclusters on the nanowire surface $d$ )

Figure 6.11: TEM image of titania-coated $\mathrm{SnO}_{2} \mathrm{NWs}$ after cycling at showing nanowire morphology with hollow structure

Figure 6.12: HR-TEM of titania-coated $\mathrm{SnO}_{2} \mathrm{NWs}$ after $1^{\text {st }}$ cycle showing presence of tin nanoclusters on nanowire surface 
Figure 6.13: SEM, TEM (inset) images of thin layer of titania-(a), alumina-(b) coated $\mathrm{SnO}_{2}$ NWs after cycling. The white spot as Sn clusters are presence in either titania- or alumina-coated $\mathrm{SnO}_{2} \mathrm{NWs}$

Figure 6.14: EDS data of $5 \mathrm{~nm}$ titania-coated $\mathrm{SnO}_{2} \mathrm{NWs}$ after cycling 


\section{LIST OF TABLES}

Table 1.3.1: Material quantities incorporated into cell windings of 48-cell power assist HEV battery

Table 3.4.1: Summary of the experimental conditions

Table 4.2.1: Effect of particle size on terminal settling velocity

Table 4.4.1: Experimental conditions for the study of effect of hydrocarbon

Table 4.7.2: Catalysts- composition, BET surface area and crystallite size

Table 5.5.1: Summary of the experimental conditions used for the nanowire production

Table 6.1: $1^{\text {st }}$ cycle electrochemical performance comparison of pure $\mathrm{SnO}_{2} \mathrm{NWs}$ and titania-coated $\mathrm{SnO}_{2} \mathrm{NWs}$

Table 6.2: Young's and bulk modulus of alumina and titania 


\section{CHAPTER I}

\section{INTRODUCTION}

\subsection{Nanotechnology today}

Following the introduction of carbon nanotubes by Iijima in 1991 [1], there has been an enormous interest in nanotechnology as evidenced by a huge investment of approximately $\$ 19.4$ billion in nanoscale science, engineering, and technology from FY2001 through FY2014 by the United States government [2]. Nanotechnology deals with variety of things ranging from the science and engineering to the applications of nanomaterials. Nanomaterials are composed of crystals with one of the dimensions to be less than $100 \mathrm{~nm}$ in length. Nanomaterials can be grouped into zero-(0D), one- (1D) or two-dimensional (2D) nanomaterials. The dimensional designation is defined as the number of dimensions that allows motion of transport carrier. 0D nanomaterials include nanoparticles, quantum dots, or nanocubes and 2D nanomaterials refer to thin films. The most desired nanostructures are 1D nanomaterials that comprise of nanowires, nanorods, nanotubes, nanofibers, nanoribbons, nanobelts and nanoneedles, etc. In contrast to bulk materials, reduction of grain sizes in nanomaterials provides unusual changes in both chemical and physical properties such as fast carrier transport, short diffusion length, and 
good strain relaxation[3]. Throughout the development and manipulation of these nanomaterials over last two decades, scientists and engineers have been able to bring nanomaterials into very promising opportunity and applications in energy, environment, health, medicine, transportation, communications, military, security, etc.

\subsection{Applications of metal oxide nanowires}

A recent report in Nature has quoted that "Nanowires, nanorods or nanowhiskers. It doesn't matter what you call them, they're the hottest property in nanotechnology"[4]. Indeed, nanowires or 1D nanomaterials have attracted great research interest due to unique properties that allow them to be ideal building blocks in important applications, such as catalyst supports [5, 6], absorbents [7], batteries [6], solar cells [8], capacitors, sensors [9], and polymer composites [10]. In particular, metal oxide nanowires have emerged as one of the most important class of 1D nanomaterials - as they tend to be single crystalline with well-defined surfaces, they possess excellent surface and bulkcharge-carrier-transport properties [11].

Currently, most technological applications are using bulk materials, though there are a few emerging applications using nanoparticles due to their availability and ease of manufacturing in large quantities. All of the applications using nanowire materials have been demonstrated only at lab scales and a full commercial device prototype using nanowire materials has not been realized yet. Charge transport in nanoparticles, shown in Figure 1.2.1a, is usually very poor due to electron hopping through the inter-particle network. Nanowires of the same material usually exhibit much better charge transport properties due to their single crystalline pathways for both surface and bulk transport (Figure 1.2.1b). They also can provide better strain relaxation for volume expansion 
during charging/discharging in lithium ion batteries. Thus, the use of nanowires can enhance performance by at least an order of magnitude. For instance, the use of tin oxide nanowire-based anodes has been reported to improve capacity retention in lithium-ion batteries [6].

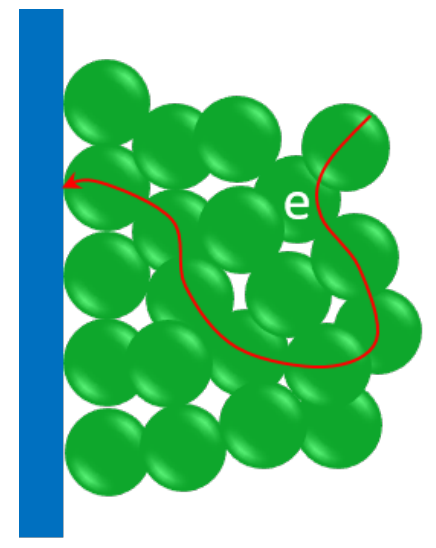

a

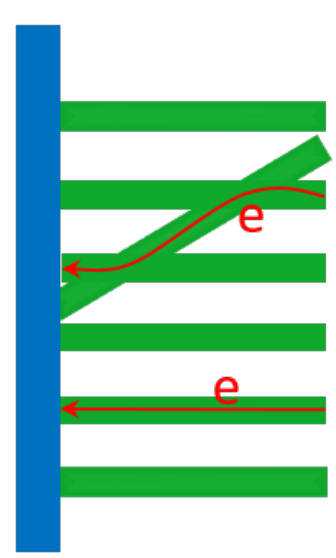

b

Figure 1.2.1: Schematic of electron transport through a) nanoparticles and b) nanowires

In terms of catalysis applications, nanowire based materials are providing multifold advantages for catalyst design. The single crystal nature and faceting improve the properties of the supported catalytically-active metals through the resulting metal cluster morphology. The well-defined surface facets provide higher concentration of desired sites [12]. Especially, nanowire support undergoes less sintering than the conventional supports of bulk or spherical particles $[7,13]$. As shown in Figure 1.2.2, nanowire support shows much less sintering than conventional support of particles thus maintaining high surface area for active sites for catalytic activity. Recently, zinc oxide nanowires have been shown to be an excellent catalyst support for ultra-deep 
hydrodesulfurization of diesel fuel. The advanced catalyst of $\mathrm{Ni}$ metal supported on zinc oxide nanowires could lower the sulfur content from $200 \mathrm{ppm}$ to $1 \mathrm{ppm}$ [14].

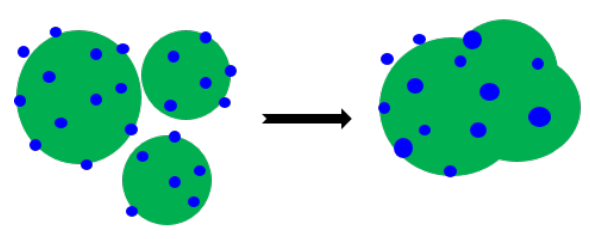

a

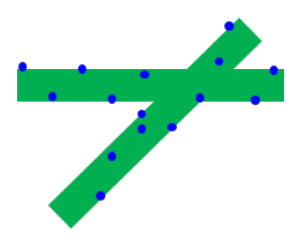

$\mathrm{b}$

Figure 1.2.2 Schematic of sinterability of a) conventional nanoparticles and b) nanowire powders.

\subsection{Motivation for this study}

The motivation for this study on scalable methods for producing metal oxide nanowires is based on two primary reasons: (1) critical need for obtaining large amounts of nanowires for realizing any industrially relevant application; and (2) lack of both scalable concepts and reactors for producing any nanowire materials such as metal oxide nanowires.

As mentioned earlier, all of the applications using nanowire materials have been shown only at lab scale and a full commercial device prototype using nanowire materials has not been realized yet because there is no such amount of nanowire materials that is enough for even a simple testing and prototyping. For instance, in order to build a 48-cell power assist HEV battery, one would need $4.8 \mathrm{~kg}$ of active materials for anode and $7.5 \mathrm{~kg}$ of active materials for cathode (Table 1.3.1). The requirements for catalysis also require large quantity production at a scale of hundreds of tons per year. Thus, a simple and 
facile method for bulk production of nanowires is required to fully utilize the advantages of nanowires over bulk and nanoparticles.

Table 1.3.1: Material quantities incorporated into cell windings of 48-cell power assist HEV battery. Adapted from Ref. [15]

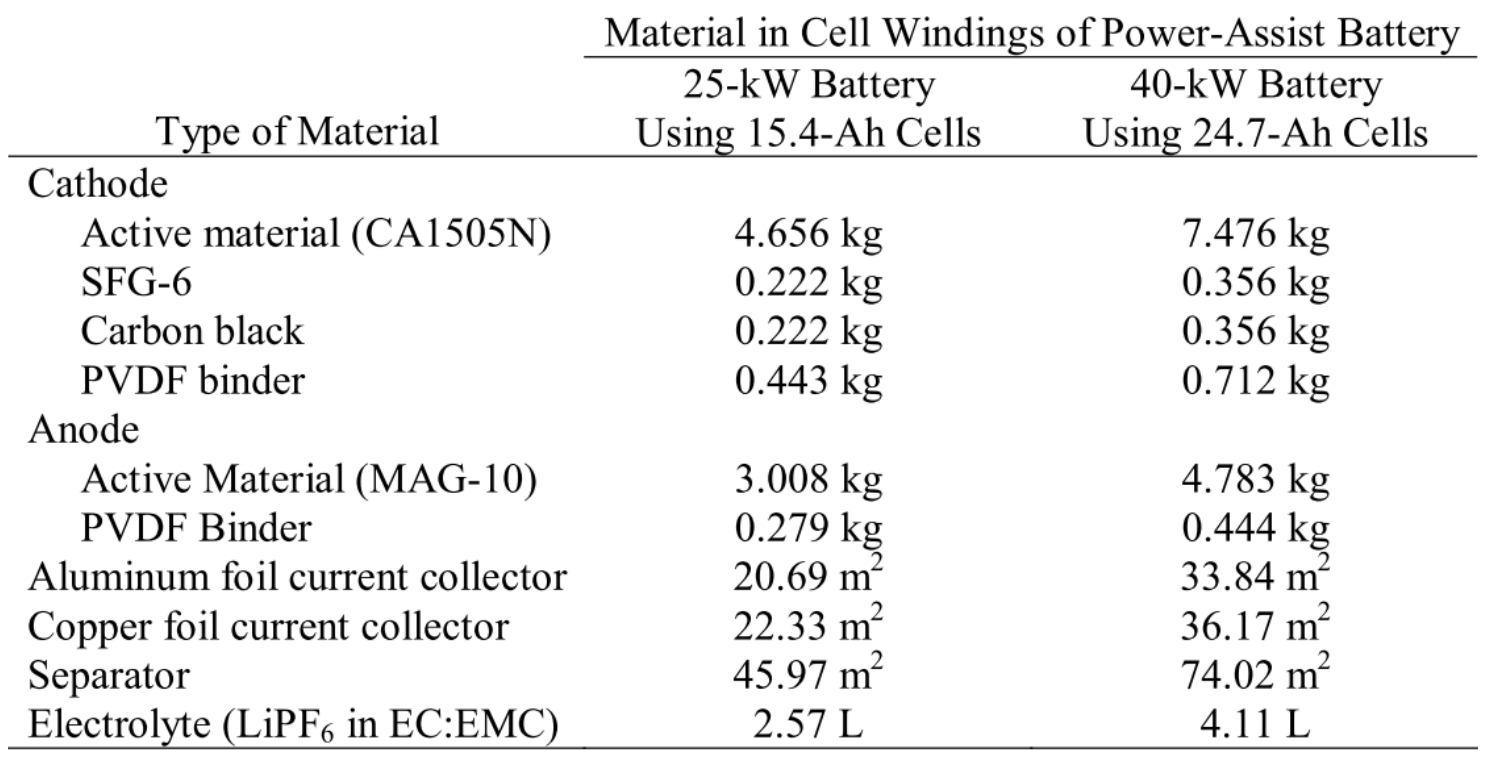

Even though metal oxide nanowires have been paid extensive investigation over the past two decades, practical applications based on metal oxide nanowires are still challenging due to a limited scalability of current methods for producing nanowires. The state of the art methods for producing nanowires are traditional liquid phase methods such as hydrothermal synthesis, and traditional gas phase methods such chemical vapor deposition. Unfortunately, they are all limited to produce only a few hundred milligrams of nanowires and are all not scalable or difficult to scale up. Therefore, a generic scalable method to produce metal oxide nanowires at kilograms' scale or commercial scale has both scientific and technological significance. 


\subsection{Proposed concepts for scalable production and uses for metal oxide nanowires}

Sunkara's group has developed two scalable techniques for producing 1D nanomaterials including direct oxidation and solvo-plasma oxidation[16, 17]. Direct oxidation involves formation of metal oxide nanowires in gas phase by reacting micro size metal powders with plasma flame containing oxidizer species such as oxygen radicals (Figure 1.4.1 a). Accordingly, nanowire production experiments were conducted using a downward atmospheric microwave plasma reactor [18]. However, this reactor has certain limitations in terms of scalability. The reason for this limited scalability is due to plasma instability and extremely short residence time of 0.01 second induced by drag force exerted on the particles. In this dissertation, a fluidized bed reactor is proposed for large scale production of metal oxide nanowires in direct oxidation scheme. The key feature of this design is long residence time, which is due to fluidizing phenomena of starting materials inside the reactor chamber. A stainless steel reactor will be equipped with either a plasma flame or hydrocarbon flame at the bottom (Figure 1.4.1 b). The reactor is also equipped with cyclone and bag house filter enabling efficient powder collection and allows continuous production. The zinc oxide nanowire production will be studied using a fluidized bed using both microwave plasma and hydrocarbon flames. 


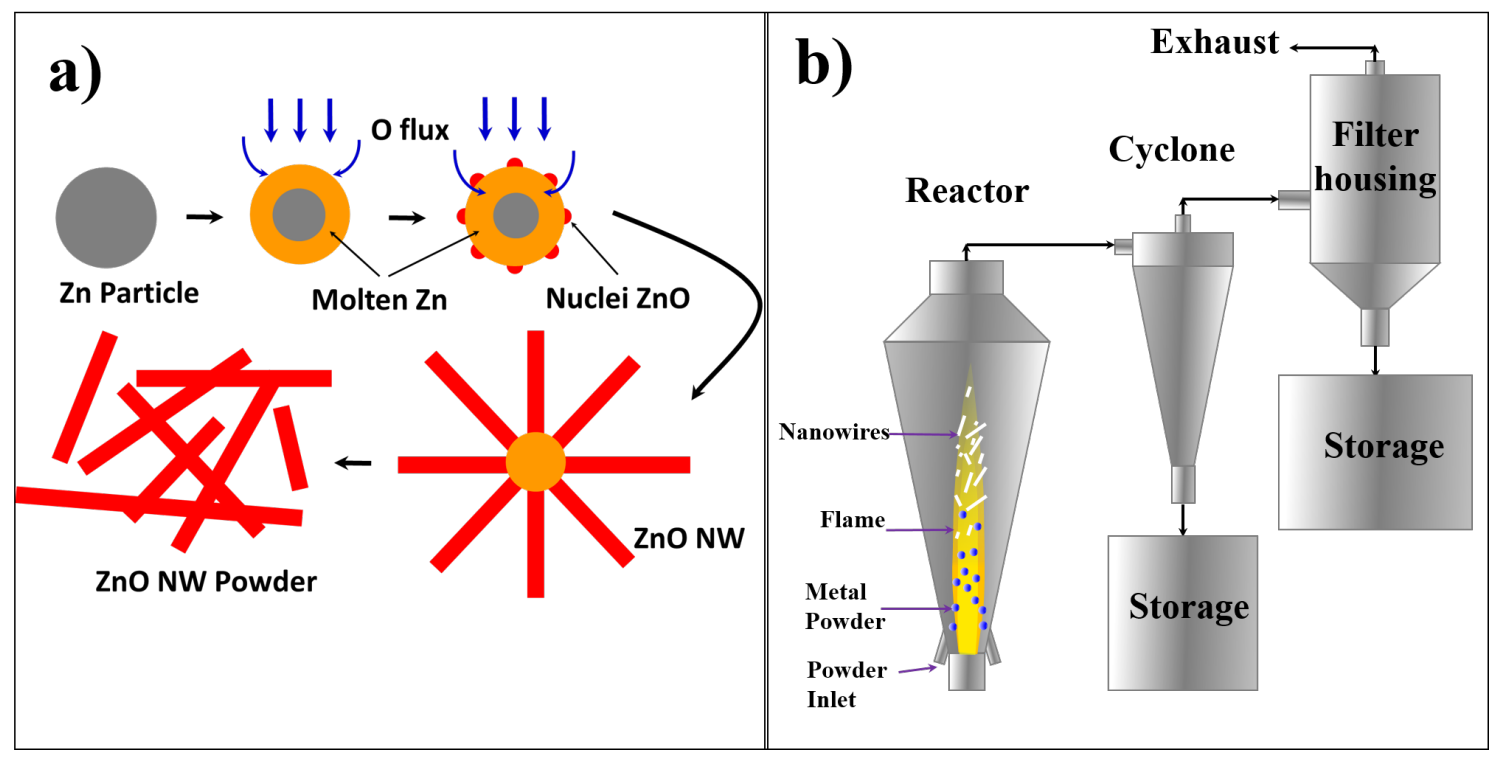

Figure 1.4.1: a) Schematic of direct oxidation of metal powder and b) proposed reactor setup

The above concept of direct gas phase oxidation has certain limit for high melting point metal like titanium, manganese, and tungsten. Experiments with these high melting point metals usually resulted in nanoparticles instead of nanowires[18]. Using the second technique, termed as solvo-plasma oxidation, metal oxide nanowires could be produced in very short time scale of 1 to 5 minutes[16]. Hence, tin oxide nanowires will be synthesized using solvo-plasma oxidation. The growth mechanism for the case of tin oxide will be examined to confirm the generic aspects of solvo-plasma method for producing other metal oxide nanowires. The scalability of solvo-plasma technique will also be studied. 


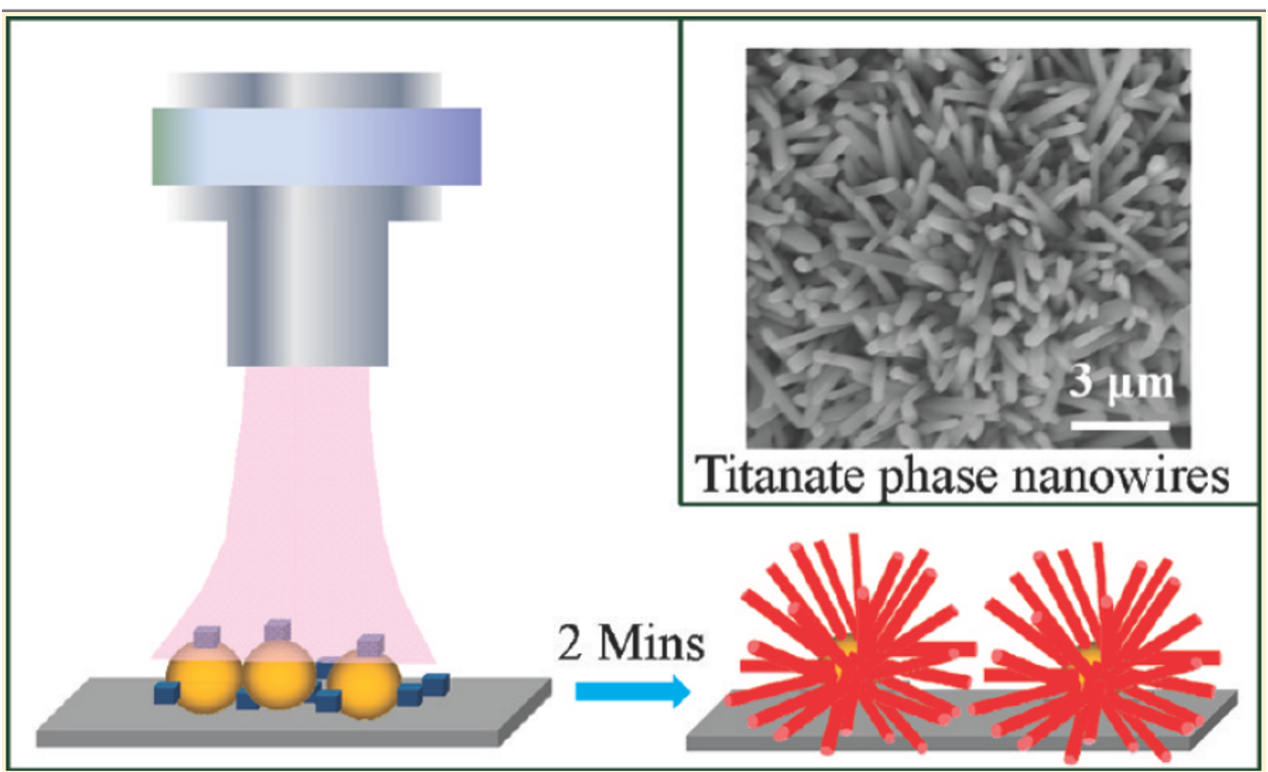

Figure 1.4.2: Schematic of solvo-plasma oxidation of titania nanowire arrays and powder. Adapted from Ref. [16]

Tin oxide nanowires attract great interest for lithium ion battery applications. However, as-produced tin oxide nanowire powders are not chemically stable with respect to cycling. Here, the tin oxide nanowires will be coated with ultra-thin layers of titania or alumina coating as thin as $1 \mathrm{~nm}$ to understand the stability with respect to lithium ion cycling.

Nanowire based materials are providing several advantages in catalyst application such as low sinterability and high surface area. However, there are no studies on nanowire based materials for catalyst support applications yet due to non-availability of such methods for producing nanowire materials at large quantities. Hence, the bulk produced zinc oxide nanowires will be studied as catalyst support for hydrodesulfurization of diesel.

\subsection{Objectives of this study}

The overall objective of this dissertation is to study the feasibility of scalable 
methods for producing nanowires in bulk quantities. Several nanomaterials such as titania, alumina, and fumed silica have been commercial produced using flame spray pyrolysis[19]. However, this method does not work for producing metal oxide nanowires. In some random experiments, zinc oxide nanorods can be produced but it is difficult to control their formation or it requires expensive starting materials.

Even though, the concept of oxidation of micron scale metal powders has been shown to produce metal oxide nanowires specifically in the case of Zinc and related lowmelting metals. However, there are several challenges exist with scale-up of the technique and these challenges include understanding of the role of residence time for oxidation and time scale for fluidization and the corresponding engineering designs for reactors. In addition to the use of highly reactive oxygen radicals from plasma sources, it is also important to understand whether the oxidation of metal particles using hydroxide radicals will result in nanowire growth without vaporizing metal particles.

Similarly, the concept of solvo-plasma oxidation scheme involving oxidation of metals and metal oxides in the presence of alkali salts is interesting for producing nanowires. However, very little is understand about the generic aspects about this concept both in terms of its applicability to a variety of materials systems including low-melting metals and the underlying mechanism responsible for one-dimensional growth. Such rationalization is important for making this technique applicable for many other systems and also toward scale-up using the above mentioned fluidization schemes.

So, the main objectives of this dissertation are the following:

- Investigate limitations on using downstream atmospheric microwave reactor for producing zinc oxide nanowires using direct oxidation scheme. Study the 
properties of produced zinc oxide nanowires in an industrially relevant application such as catalyst supports.

- Investigate the use of custom-designed, upward flow and fluidized reactor with production of zinc oxide nanowires using direct oxidation of zinc powder. Also, investigate the feasibility of using hydrocarbon flames instead of microwave plasma discharges.

- Study solvo-plasma oxidation scheme for producing tin oxide nanowires and study the underlying mechanisms responsible for one-dimensional growth under this technique. Study the concept with other materials systems and versatility of the concept and also scalable production using fluidized bed reactor.

- Study the bulk produced tin oxide nanowires and ways to improve their stability with cycling in lithium ion battery application.

\subsection{Dissertation outline}

This dissertation comprises of eight chapters. Chapter 1 introduces 1D nanomaterials and their applications, the need for development of scalable method for producing 1D nanomaterials at large scale, and objective of the work. Chapter 2 reviews the state of the art methods for producing nanomaterials with an emphasis on nanowires and nanorods of metal oxide such as tin oxide and zinc oxide. The reactor design and assembly, methodology and experimental, and material characterization used in this work will be described in Chapter 3. Chapter 4 describes the results with zinc oxide nanowires produced using fluidized bed reactor. An investigation on understanding of nanowire morphology control and process optimization for high yield is detailed. A discussion of zinc oxide nanowires as catalyst support for hydro-desulfurization of diesel is also given. 
Chapter 5 talks about a scalable solvo-plasma method for producing tin oxide nanowires. A focus on growth mechanism and scalability is presented. Chapter 6 discusses solving the problem with volume expansion and initial capacity loss of nanowire based anodes for lithium ion battery application. Finally, Chapter 7 summarizes the work and Chapter 8 gives recommendation for future work.

\subsection{Impact of this dissertation}

The state of the art for nanowire synthesis is limited to produce only a few milligrams to a few hundreds grams which is not sufficient for any industrial applications. A facile, reliable, and scalable method for nanowire production will allow prototyping and designing a near commercial scale reactor for industrial applications. Eventually, economical manufacturing methods for nanowire materials are crucial for the US to realize the impact of nanotechnology as a goal of the Launch of National Nanotechnology Initiative in 2000 [2]. 


\section{CHAPTER II}

\section{BACKGROUND}

\subsection{Introduction}

0D nanomaterials are the easiest form of nanostructured materials that could be produced in bulk quantities. Commercial production of several nanoparticles at ton-scale such as carbon black, fumed silica and titania has been reported using salt solutions as precursor by classical flame or flame spray pyrolysis [20]. The formation of nanoparticles is straightforward and simple. The salt precursor is usually sprayed as small droplets into the combustion chamber for oxidation. The mechanism involves the oxidation of precursor in gas phase to form metal oxide molecules or nuclei. The metal oxide molecules/nuclei undergo coagulation, sintering and surface growth to form metal oxide nanoparticles. The small metal oxide nanoparticles can be aggregated to form group of nanoparticles. In other words, nanoparticle growth occurs in all of the dimensions surrounding the nuclei. Figure 2.1.1 showed a typical mechanism responsible for formation of titania nanoparticles using titanium tetraisopropoxide (TTIP) solution precursor by spray flame pyrolysis. 


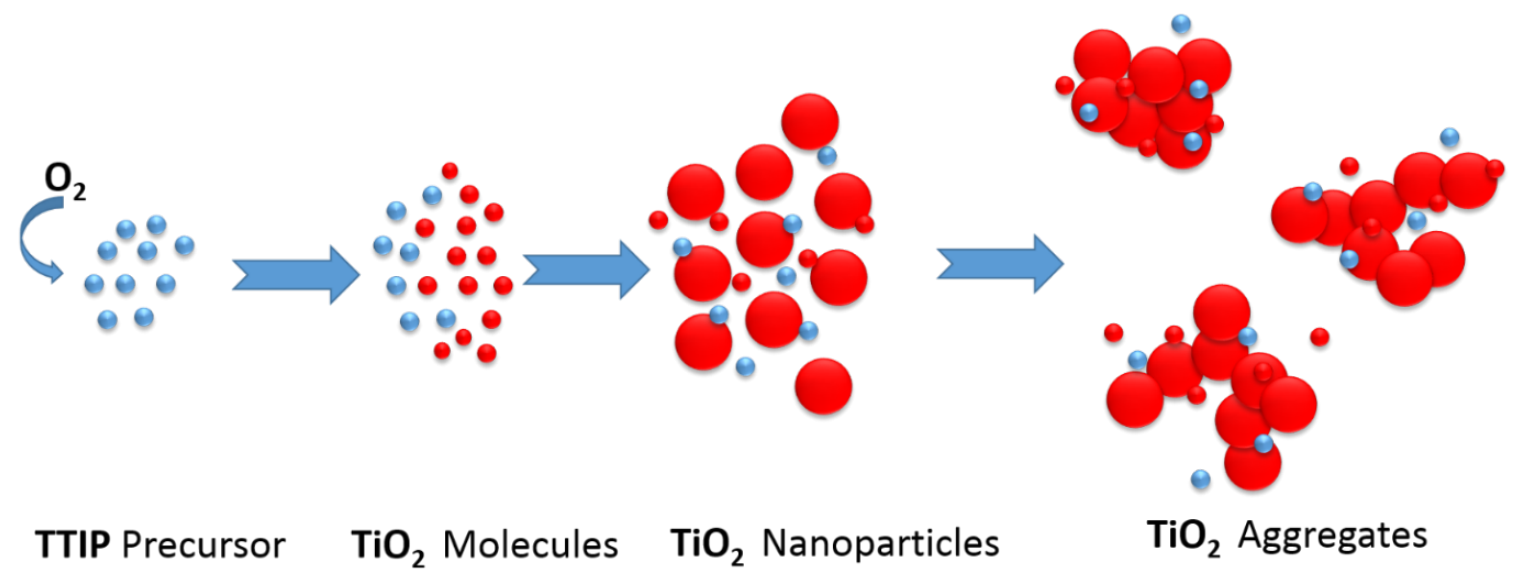

Figure 2.1.1: Schematic formation of titania nanoparticles in gas phase using TTIP precursor

Cylindrical shape likes 1D nanomaterials are highly desired nanostructured materials compared to $0 \mathrm{D}$ nanomaterials. However, the formation of $1 \mathrm{D}$ nanomaterials differ significantly from that of nanoparticles. To form a $1 \mathrm{D}$ nanomaterial, the growth must occur preferentially in one dimension. Vapor-liquid-solid (VLS) is one of the first growth mechanism proposed by Wagner et al. [21] for growth of 1D nanomaterials assisted or catalyzed by the use of gold clusters. The reaction kinetics include four steps: (1) mass transport of species in the gas phase to surface of molten metal particles; (2) chemical reaction among species at the vapor-liquid interface; (3) diffusion of species through the molten metal particles; (4) incorporation of supersaturated species into 1D nanomaterials[22]. The selection of metal particle catalyst is crucial for 1D growth. Typically, an expensive metal like Au is used.

Since, there has been an increasing attention to 1D nanomaterials, a large number of techniques for 1D growth have been investigated. These techniques can be grouped into two classes: top-down and bottom-up methods. Top-down methods include ball 
milling, and etching. However, the top-down methods are typically used for micro/nanoparticle production or feature patterning in semiconductor industry which is not efficient for producing 1D materials at large scale. Bottom-up methods can again be classified into liquid phase and gas phase techniques. Liquid phase techniques usually use water or organic solvent as growth media. Gas phase techniques are approaches where the growth occurs in gas phase. The following discussion gives an overview of bottom-up methods for 1D growth of metal oxides. There will be more focus on reviewing prior work on the production methods for zinc oxide and tin oxide nanowires or nanorods. The plasma based techniques and hydrocarbon flame based techniques are also covered in more detail.

\subsection{Liquid phase techniques}

Liquid phase techniques have been widely used for synthesis of 1D nanomaterials. Liquid phase approaches have several advantages such as a simple reactor, low cost, and low temperature. These include precipitation, hydrothermal, solvothermal, so-gel, and electrochemical.

\subsubsection{Precipitation method}

Precipitation is a widely used method for synthesizing $0 \mathrm{D}$ and $1 \mathrm{D}$ nanomaterials, particularly metal oxide nanowires and nanorods. The precipitation involves formation of precipitates by hydrolysis of metal salt in basic $\mathrm{pH}$. The precipitates may remain in suspension. Therefore, a centrifugation step is required to separate the precipitates. The washing, calcining and milling steps are followed to obtain the nanomaterial product. The synthesis of 1D nanomaterials such as nanowires or nanorods often requires the use of a catalytic seed layer. The precipitation is commonly controlled by $\mathrm{pH}$ of solution, reaction 
time and temperature.

Zinc oxide nanorods have been prepared by Terasako et al. using precipitation method at $90{ }^{\circ} \mathrm{C}$ on $\mathrm{CuO}$ substrate coated with $\mathrm{Au}$ film as seed layer [23]. Zinc nitrate was used as precursor and hexamethylenetetramine was used to control the $\mathrm{pH}$. It found that the nanorod length increases with increasing concentration of zinc nitrate. A similar study done by using Al-Asadi et al. for zinc oxide nanowires [23]. The zinc oxide nanoparticles were used as seed layer. Zinc oxide nanowires of $45 \mathrm{~nm}$ in diameter and 11.3 microns in length were obtained after 16 hours of reaction time.

\subsubsection{Hydrothermal and solvothermal methods}

Hydrothermal and solvothermal methods are carried out in a solution containing solvents and a soluble metal salt precursor. Solvent for hydrothermal synthesis is water while the solvothermal requires an organic solvent such as ethylene glycol. A Teflonsealed stainless steel autoclave is used as a reactor where solutions of solvent and precursor are gradually heated up to $100-300{ }^{\circ} \mathrm{C}$ at pressures higher than 1 bar for duration spanning several hours to days. The $1 \mathrm{D}$ growth involves nuclei formation from a supersaturated solution. The morphology, crystallinity, and purity of resulting 1D nanomaterials can be controlled by changing the temperature, pressure, solvent, surfactant, precursor concentration, $\mathrm{pH}$ of solution or template.

For example, Cheng et al.[24] reported a hydrothermal method for zinc oxide nanowire arrays using zinc acetate as zinc precursor and hexamethylenetetramine as hydroxide source. A layer of zinc oxide particles of $5 \mathrm{~nm}$ in diameter was used as seeds

on $\mathrm{Si}$ substrate. The synthesis was carried out at $90{ }^{\circ} \mathrm{C}$ for 60 minutes followed by 
washing and baking. Zinc oxide nanowires also were hydrothermally synthesized without using any seed [25]. The nanowires were shown to be $30-50 \mathrm{~nm}$ in diameter and $200-$ $600 \mathrm{~nm}$ in length. The nanowires were then functionalized by Au nanoparticles for gas sensor application.

Doping during liquid phase synthesis is usually a challenge as the dissolution of dopant into the solution occurs before getting incorporated into the host materials. By mixing the precursor solution of zinc nitrate and manganese nitrate, Mn-doped $\mathrm{ZnO}$ nanorods have been successfully synthesized using hydrothermal method. The incorporation of Mn was reported to change shape, size and density of the nanorods [26].

In a recent study, Pal et al.[27] showed gram-scale synthesis of various tin oxide nanostructures including nanowires. $\mathrm{SnCl}_{4} \cdot 4 \mathrm{H}_{2} \mathrm{O}$ and $\mathrm{NaOH}$ were used as precursor for the synthesis in an autoclave at $180-225{ }^{\circ} \mathrm{C}$ for 12 or 24 hours. There was no surfactant used. The shape of resulting tin oxide was highly influenced by $\mathrm{pH}$, in which tin oxide nanoparticles were produced at lower $\mathrm{pH}$ (5-7) while tin oxide nanowires were produced at higher $\mathrm{pH}$ of greater than 9.

Solvothermal synthesis is similar to hydrothermal synthesis except the fact that non-aqueous solvent is used. Tungsten oxide nanorods have been synthesized via solvothermal approach at $200{ }^{\circ} \mathrm{C}$ for 9 hours using ethylene glycol as solvent. Ethylene glycol was believed to play important role as soft template in the formation of nanorods[28].

Many other metal oxide nanowires such as $\mathrm{TiO}_{2}[29], \mathrm{MnO}_{2}$ [30], $\mathrm{Co}_{3} \mathrm{O}_{4}$ [31], $\mathrm{CuO}[32], \mathrm{WO}_{3}[28,33]$ were also successfully prepared using hydrothermal or solvothermal synthesis. Even though, a large number of metal oxide nanowires can be 
synthesized via hydrothermal technique, the 1D growth mechanism is not well understood. Long reaction time scale up to a day is one of the facts that make hydrothermal/solvothermal unsuitable for large scale production of 1D nanomaterials. The other issue with these methods include the use of high volumes of solvent in a pressurized autoclave.

\subsubsection{Electrochemical and sol-gel syntheses}

Electrochemical and sol-gel methods are usually carried in templates for 1D growth. The templates serve as a scaffold for 1D materials growth. The template will be removed by selective etching. The electrochemical synthesis is carried out in a standard electrochemical cell. A template works as a working electrode where 1D growth occurs. The working, counter, and reference electrodes are immersed in the electrolyte of the electrochemical cell. The electrolyte is usually a salt solution of the metal which is the source for depositing desired metal oxides on the working electrode. The deposition rate can be controlled by potential and current applied between electrodes, concentration of electrolyte and material nutrient. The diameter and length of nanowires can be controlled using template dimmesions. Track-etch membranes (TEM), anodic alumina membranes (AAM), or diblock copolymer are widely used as templates for electrochemical and solgel synthesis. For example, zinc oxide nanowires were synthesized by electrodeposition of zinc nitrate in AAM template [34]. In another study, Lai et al. reported a preparation of tin oxide nanotubes by electrochemical deposition technique using TEM template [35]. The electrochemical deposition is relatively fast method due to fast driving force induced by applied potential. 
Sol-gel as a liquid phase method has been widely used for nanostructure synthesis including 1D nanomaterials of metal oxides. The sol-gel process involves the hydrolysis followed by condensation of precursor solution to form colloidal solution (sol). The sol solution is further converted to solid wet gel. The alkoxides are typical precursors for solgel synthesis. AAM is commonly used as template in sol-gel. For example, polycrystalline zinc oxide nanowires and nanotubes have been synthesized by sol-gel method using AAM as template[36]. Sol-gel is a simple method in which no major equipment is used. However, it is relatively slow because of slow diffusion of sols in solution and template. The deposition rate can be increased by heating the template or applying electric filed to enhance the diffusion of sols.

In any case, the use of a template in electrochemical or sol-gel method suffers from two major drawbacks: (1) template fabrication and (2) removal of template. To overcome these drawbacks, researchers have utilized thin seed layer of $\mathrm{ZnO}$ or $\mathrm{Au}$ for the synthesis of zinc oxide nanorods using template-free electrochemical deposition[37, 38]. Recently, zinc oxide nanowires or nanotubes have been grown using template-free electrochemical deposition using zinc foil as anode[39]. These techniques involving catalytic seed layer and templates could be useful for studies involving 1D nanomaterials at small scale but not for applications that require large quantities beyond kilogram scale for commercial implementation.

\subsubsection{Microwave assisted synthesis}

As reviewed previously, all of the liquid phase techniques require relatively long reaction time scale which can typically be anywhere from several hours to days long. In 
all of those techniques, heat transfer occurs among materials by conventional conduction, convection and radiation, which can lead to a temperature gradient between bulk and surface. The microwave irradiation heating, on the other hand, offers a unique heating mode due to the use of microwaves. The microwave field causes an internal friction among molecules in dielectric materials, which results in lower activation energy, uniform temperature from bulk and surface, and eventually faster growth of nanomaterials[40].

Microwave assisted synthesis can apply to all of the liquid phase synthesis techniques by replacing the conventional heating source by microwave irradiation heating. For example, the reaction time for the synthesis of zinc oxide nanorods have been reduced significantly to about 2 minutes using microwave irradiation heating [41]. The growth rate for zinc oxide nanowire synthesis has also been increased up to 75-100 $\mathrm{nm} /$ min using microwave assisted hydrothermal method, which is much higher than that of a conventional hydrothermal method[42]. Krishna et al. has shown that the microwave assisted hydrothermal synthesis results in higher yield in shorter time compared with the conventional hydrothermal synthesis for the case of tin oxide nanoparticles[43]. In another study, tin oxide nanotubes have been synthesized using microwave assisted hydrothermal synthesis where the reaction time was reduced significantly [44].

\subsubsection{Summary of liquid phase techniques}

Liquid phase techniques have several advantages such as simple equipment, low cost, and low temperature. However, there are lots of challenges that limit their scalability. They require the use of a large amount of solvent which can be either water in 
the case of techniques such as precipitation, hydrothermal, electrochemical or expensive, toxic organic solvent in the case of techniques such as hydrothermal and solvothermal. Water or toxic organic solvent waste may cause a serious issue with the environment. They also need lengthy reaction times of a few hours to even days in batch mode which results in low production capacity of no more than gram quantity per day. The batch mode operation and a sealed autoclave as reactor make it difficult for production scaling up. Additional steps such as washing and calcining are commonly required for liquid phase techniques. In any method using templates, two additional steps of fabricating and removing template are also required. In summary, liquid phase techniques are not suitable methods for large scale production of 1D nanomaterials.

\subsection{Gas phase techniques}

Gas phase synthesis techniques have been widely used for synthesizing 1D nanomaterials in various materials systems. The gas phase synthesis techniques have shown advantages over liquid phase synthesis techniques. Vapor-liquid-solid (VLS) is the primary growth mechanism proposed by Wagner et al. [21] for 1D nanomaterials. The mechanism is depicted in Figure 2.3.1. Briefly, gas phase species of desired nanowires are adsorbed and dissolved into molten metal catalyst to form molten alloy droplets. Continuous dissolution of gas phase species into molten metal catalyst results in the supersaturation of the desired materials in the molten alloy droplets, eventually the desired materials precipitate out from the molten alloy droplets at the solid-liquid interface in the form of nanowires [22]. This mechanism has been used extensively for the synthesis of semiconductor nanowires such as silicon, germanium nanowires or nanotubes such as carbon nanotubes. 

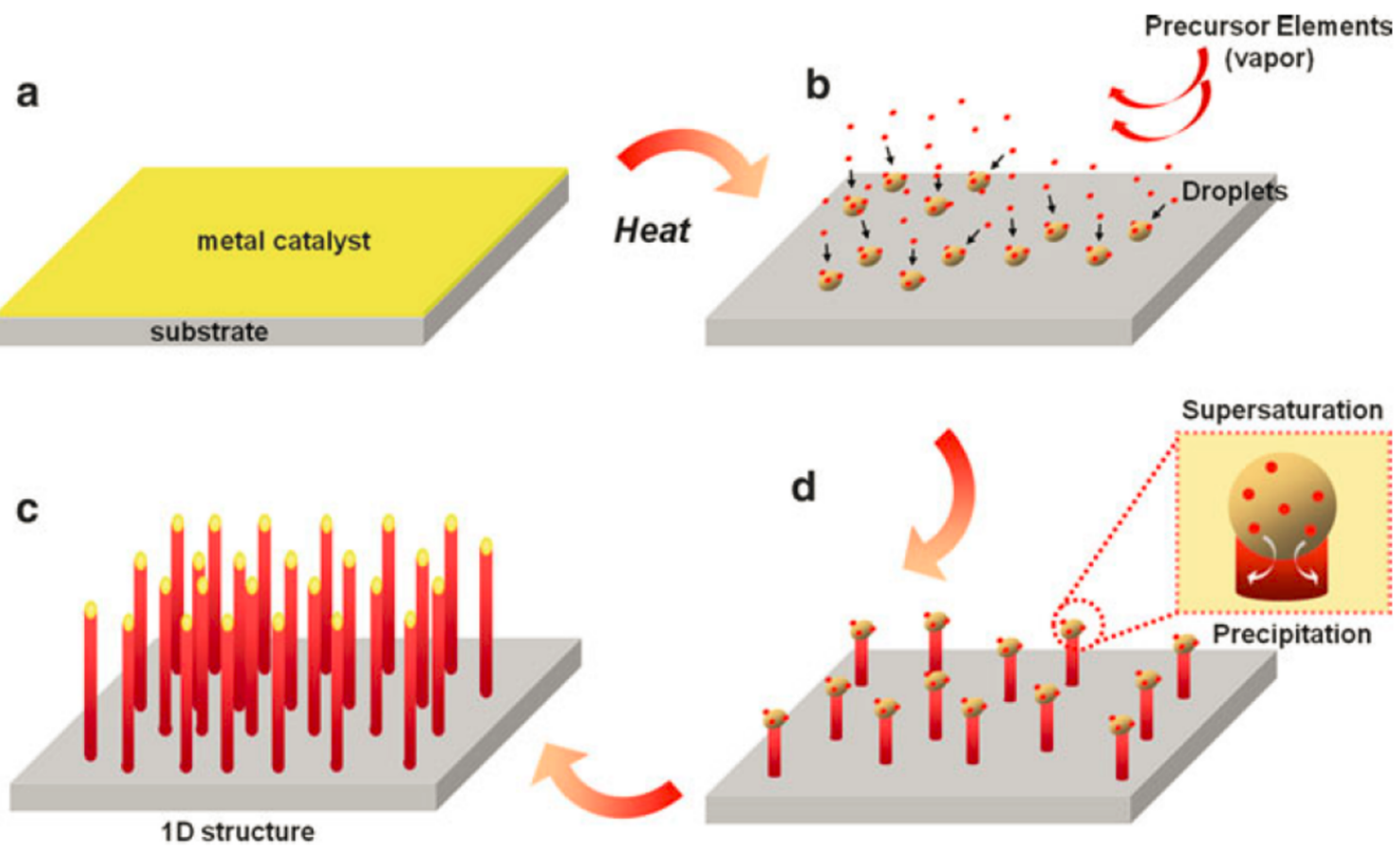

Figure 2.3.1: Schematic of 1D growth using VLS: (a) metal catalyst on substrate, (b) formation of liquid alloy droplets, (c) and (d) supersaturation leads to 1D growth by tipled growth. Adapted from ref. [22]

The growth occurs on a substrate in the presence of metal catalyst by either tip-led or basal growth. Thus, the nanowire diameter can be controlled by the metal catalyst size. Each of catalyst droplet results in a single nanowire. Typically, an expensive metal like $\mathrm{Au}$ is used. One requirement for the metal catalyst is that metal catalyst must be able to form a liquid alloy with desired species, thus the reaction commonly takes place at high temperature.

Recently, Sunkara's group has developed techniques that allows synthesizing 1D nanomaterials of low melting point metals without using any catalyst. For example, gallium oxide nanowires, nanotubes have been synthesized using gallium metal[45]. In this technique, nucleation of gallium oxide occurs on the substrate and acts as catalyst for 
further growth of nanowires, nanotubes as same as traditional VLS growth. In a sequent study, it found that several low melting point metals such as gallium, zinc, tin show low solubility for several element semiconductors. Thus, large size catalyst particles can be used to support bulk nucleation on the surface. This bulk nucleation lead to bulk growth of nanowires from a single, big metal particles[46]. Following these studies, bulk production of zinc and tin oxide nanowires have been reported, which includes direct gas phase oxidation of micron size zinc metal for zinc oxide nanowire production at hundredgrams scale[17].

\subsubsection{Chemical vapor deposition}

Chemical vapor deposition (CVD) is a traditional method that utilizes VLS mechanism for 1D growth. A tubular reactor made by quartz is typically used in CVD as shown in Figure 2.3.2. Gas phase precursor such as silane and germane for silicon and germanium nanowires respectively is preferred but solid precursor such as oxides of zinc and tin for zinc oxide and tin oxide nanowire synthesis can be used. When solid precursor is used, the substrate is placed at downstream. The vapor phase reactants are either carried into the reactor by carrier gas or vaporized from an alumina boat. The heating unit is used to control the temperature. The chamber pressure is kept at relatively lower than

100 Torr to create laminar gas flow. The variables include temperature, pressure, precursor type, precursor concentration for gas precursor, substrate configuration. 


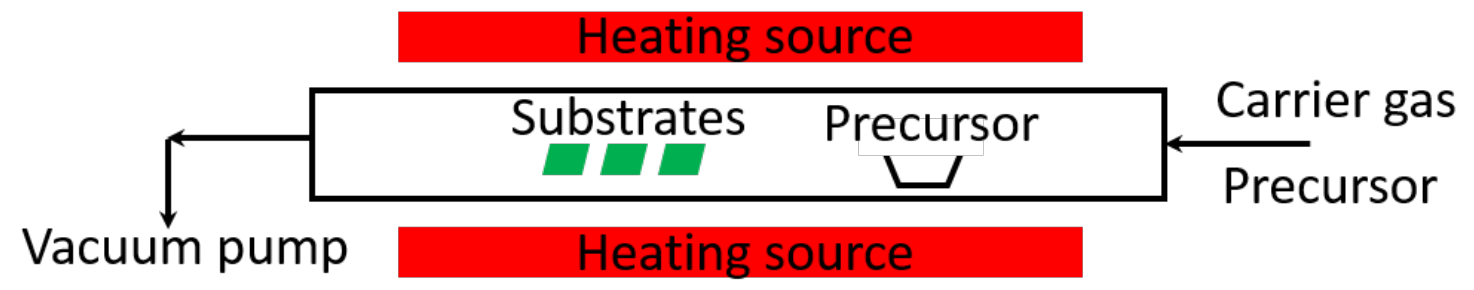

Figure 2.3.2: Schematic of a CVD reactor

For example, zinc oxide nanowires of $70 \mathrm{~nm}$ in diameter and 1 micron in length were synthesized using CVD [47]. A mixture of zinc oxide powder and graphite was used as precursor and was placed at the upstream. Argon was used as carrier gas. At high temperature, zinc oxide was reduced to zinc metal vapor by graphite. Zinc vapor reacted with oxygen on Au catalyst to form zinc oxide. The supersaturation of zinc oxide in $\mathrm{Au}$ catalyst resulted in growth of zinc oxide nanowires. It found that the dimension of the nanowires depends on the deposition time and thickness of the Au layer. Temperature is one of the most important factors in CVD that directly influences precursor evaporation, specie diffusion, and alloy formation. Yuan et al. [48] reported that the morphology evolution of zinc oxide nanostructures from zinc metal foil depends on the oxidation temperature. A thick layer of zinc oxide was obtained at the temperature lower than melting point; zinc oxide nanowires at temperatures between melting points and boiling points; and zinc oxide nano-tetrapods at the temperatures above the boiling point.

Similarly, tin oxide nanowires, indium oxide nanowires have been synthesized using CVD [49]. A thin Au film on substrate acted as catalyst. The oxides of tin and indium were mixed with carbon nanotubes to form precursors. The substrate temperature was found to be a crucial factor for controlling morphology of resulting nanostructures. Sunkara et al. presented a simple CVD for tin oxide nanowire synthesis [50]. Small tin 
balls were vaporized and transported towards the substrate as liquid droplets. The tin droplets reacted with oxygen to form tin oxide nuclei. The selective wetting of tin vapor onto tin oxide nuclei led to growth of tin oxide nanowires on quartz substrate.

The requirement of a volatile precursor ( $\mathrm{Si}, \mathrm{Ge}$, Carbon) or low melting point meal (Sn, Zn) makes it difficult to produce 1D nanomaterials of high melting point metals such as tungsten or molybdenum. A modified version of CVD which is hotwire CVD (or hotfilament CVD) can be used to produce tungsten oxide or molybdenum oxide nanowires and nanorods. As shown in Figure 2.3.3, a hotwire CVD setup is similar to traditional CVD setup except that metal filaments as precursor wound on top of ceramic rods. The metal filament is resistively heated up due to Joule heating when a voltage is applied. Tube furnace can be used to control the temperature of the substrate. Under Joule heating, oxygen reacts with hot-filament to form metal oxide vapor. By controlling the transport of metal vapors, metal oxide vapors using temperature or reactant gases, metal vapor or metal oxide vapor get deposited on the substrate in form of nanowires. For example, tungsten oxide nanowire arrays have been synthesized on quartz substrate using tungsten filament as precursor and low pressure oxygen as reactant gas[51]. By increasing the substrate temperature to $1450{ }^{\circ} \mathrm{C}$ or higher, tungsten nanowires were obtained[52].

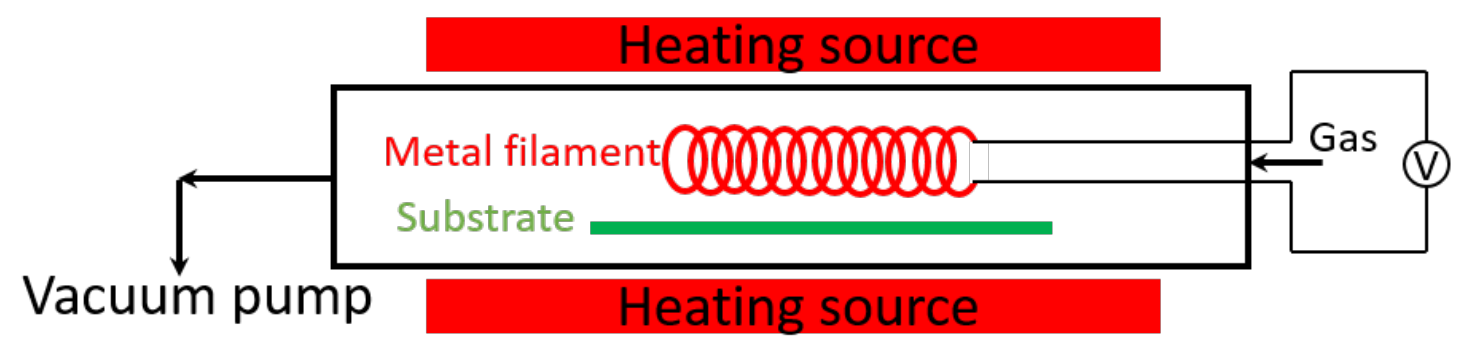


Figure 2.3.3: Schematic of a hotwire CVD reactor

Metal organic chemical vapor deposition (MOCVD) is a special case of CVD, which has been used for the synthesis of multi-element alloy. High vapor pressure based precursor such as methyl or ethyl of metal is used. The precursors are bubbled to the reactor. The simultaneous decomposition/reaction of precursors leads to formation of multi-element alloy such as $\mathrm{GaSb}_{\mathrm{x}} \mathrm{N}_{1-\mathrm{x}}[53]$.

\subsubsection{Pulsed laser deposition}

Pulsed laser deposition is basically a physical vapor deposition where the high energy laser source is used to bombard the target as shown in Figure 2.3.4. The reactor setup is quite similar to that for chemical vapor deposition, except there is a laser source. Target is made of mixture of desired materials and/or catalyst in form of cylindrical pellets. The laser causes generation of vapor, plasma, or small fragments of the target. High power laser source can generate plasma which contain highly active species such as electrons, ions, and neutrals. Vapor phase species of desired materials are transported along with carrier gas and are deposited on the substrate as nuclei. The reactor chamber is also equipped with tube furnace. The pressure and temperature are controlled in such a way that the nuclei grow into desired nanostructure such as $1 \mathrm{D}$ nanomaterials.

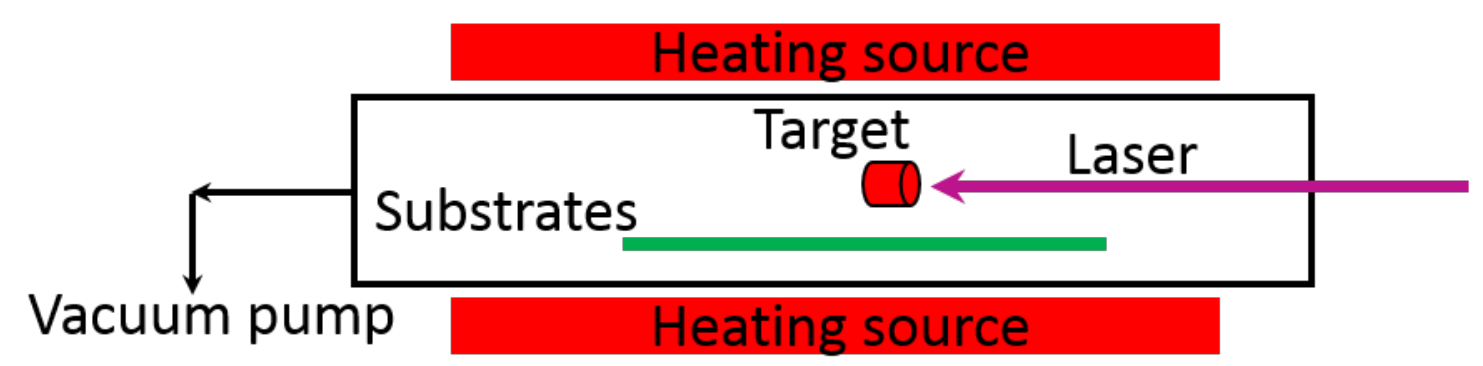


Figure 2.3.4: Schematic of reactor for pulsed laser deposition

For example, zinc oxide nanorods have been synthesized using zinc oxide pellets as targets by pulsed laser deposition[54]. Lieber et al. reported an approach for synthesizing silicon or germanium nanowires from targets contained silicon or germanium and Fe (catalyst) using pulsed laser deposition, respectively [55]. The VLS mechanism involves formation of Si-Fe liquid droplets followed by dissolution of vapor phase silicon and supersaturation of silicon in liquid droplets. Nanowire precipitates from supersaturated liquid droplets and continues growing to the end of hot zone.

\subsection{Plasma based techniques}

Plasma are partially ionized gases. It is a combination of highly active gaseous species such as electrons, ions, and neutrals. Plasmas can be broadly divided into two categories: thermal plasmas and non-thermal plasmas. Thermal plasma is a thermodynamic equilibrium plasma where all of the species have the same temperature (electron temperature $=$ ion temperature $=$ neutral temperature or $\left.T_{e}=T_{i}=T_{n}\right)$. Thermal plasma occurs at atmospheric pressure or close to atmospheric pressure[56]. There is extensive, effective transfer of potential energy to kinetic energy of gaseous particles by three-body collisions. The potential energy (ionization, dissociation, excitation to metastables) is transferred to kinetic energy of colliding particles thus heating gas. Therefore, thermal plasma is often referred as hot plasma. Thermal plasma has temperature up to $10,000 \mathrm{~K}$ and thus provide extremely high energetic environment. Nonthermal plasma is non-equilibrium plasma in which the temperature of all the species are not the same. The non-thermal plasma occurs at relatively low pressure and thus the transfer of potential energy to kinetic by three-body collisions is negligible. The heavy 
particles such as ions and neutrals cannot gain energy electric field but the electrons can be due to their low mass. So the $T_{e}$ is high (about several $10,000 \mathrm{~K}$ ) and $T_{i}=T_{n}$. Nonthermal plasma can be referred as cold plasma. The temperature of non-thermal plasma can be as low as room temperature.

Typically, there are three plasma sources including microwave plasma, inductively coupled plasma and capacitively coupled plasma. The microwave plasma employs the microwave field of $2.45 \mathrm{GHz}$ while the standard industrial RF frequency of 13.45 $\mathrm{MHz}$ are commonly used for inductively and capacitively coupled plasma. Generally speaking, all of these three plasma sources can generate thermal or nonthermal plasma depending on the pressure and plasma power.

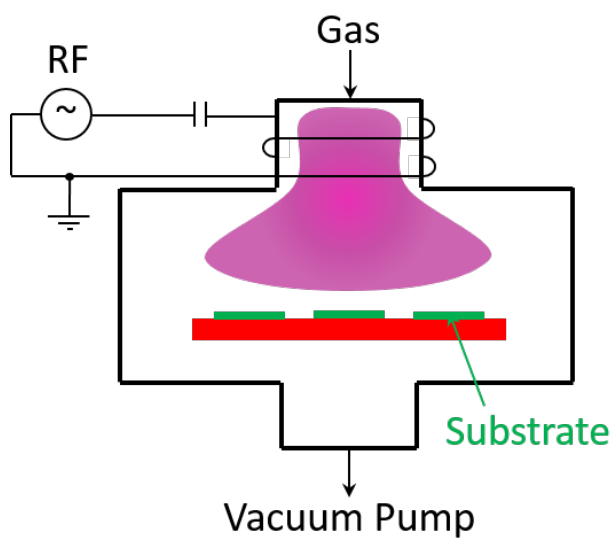

a) Inductive Coupled Plasma

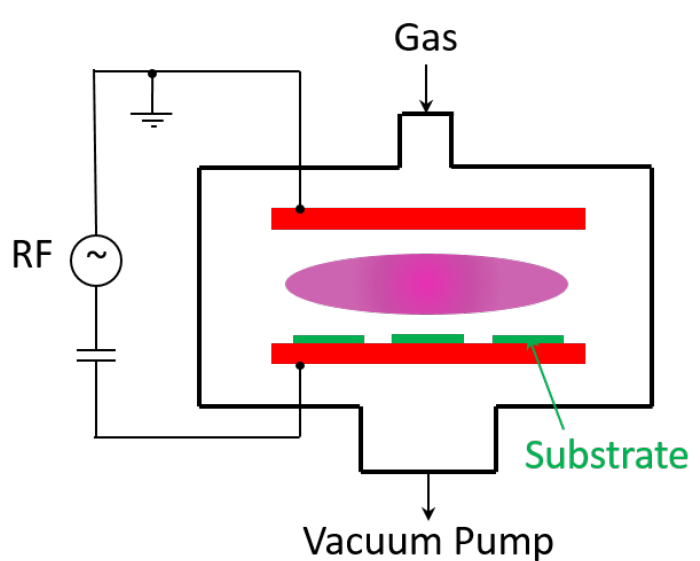

b) Capacitive Coupled Plasma

Figure 2.4.1: Schematic of inductive coupled plasma and capacitive coupled plasma

Inductively coupled plasma (ICP): As shown in Figure 2.4.1, ICP setup comprises of a RF powder supply which is to provide RF power to the coil, and a conductive coil (typically copper coil) which is cooled by water running inside the coil. 
The copper coil wraps a quartz tube of several turns. Gas is flowing to maintain the plasma. A vacuum pump is used to control the pressure. The RF voltage applied at the coil induces an alternating electric field inside the quartz tube according Faraday's Law.

Capacitively coupled plasma (CCP): As shown in Figure 2.4.1, CCP setup also consists of a RF power supply and two parallel metal plates. The RF voltage applied between metal plates generates an alternating electric field between them.

Plasma generation mechanism using ICP and CCP: When the RF generator is turned on, the background electrons present in the gas phase are accelerated in the electric field and causes an ionizing collision resulting in creating more electron. As these electrons are accelerated, causing ionization collisions so multiplication of electrons occurs. Gas is then introduced into the chamber to maintain the plasma.

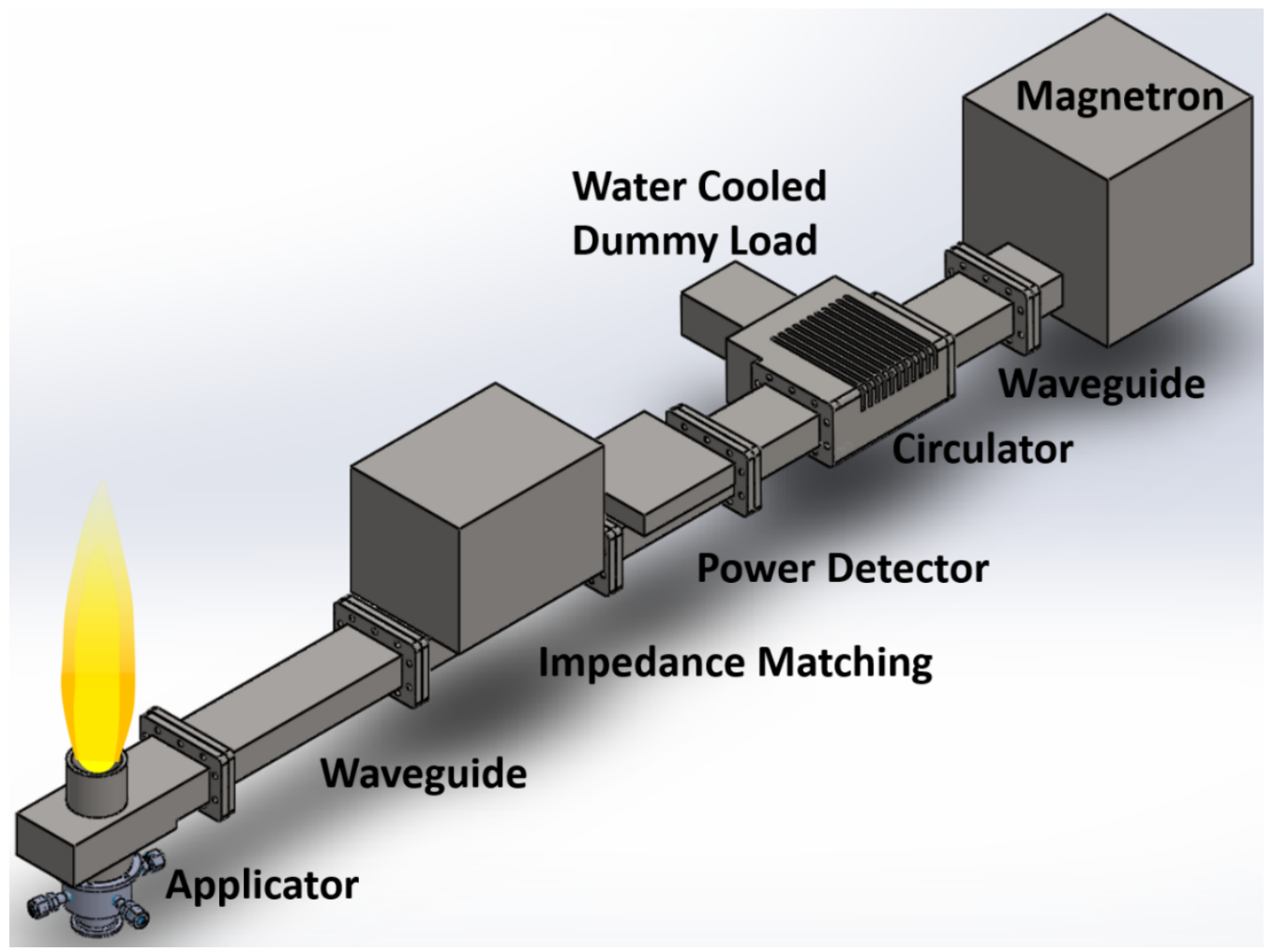

Figure 2.4.2: Schematic of atmospheric microwave plasma in Sunkara's group 
Atmospheric microwave plasma: Figure 2.4.2 shows setup of an atmospheric microwave plasma in Sunkara's group. The key component, where microwave plasma is generated, is a custom-made applicator. The detail description of all components will be explained in the next chapter. The applicator is tapered at one end where the incoming microwave will be reflected. When the generator is turned on, the magnetron generates microwave into the waveguide. Microwaves are transmitted through the waveguide to applicator. The incoming microwave interferes with reflected microwave at tapered end of microwave creating an infinite electric field at the center of a quartz tube inserted in the applicator. The infinite electric field causes dielectric breakdown of argon gas introduced into the applicator. Air or mixture of oxygen and nitrogen are then introduced into the applicator to maintain the plasma.

\subsubsection{Plasma-enhanced chemical vapor deposition}

Plasma-enhanced chemical vapor deposition (PECVD) is similar to CVD except plasma is incorporated as shown in Figure 2.4.3. Incorporation of plasma into CVD has introduced a significant amount of highly active species such as electrons, ions, neutrals into the reactor chamber. These active species help reduce activation energy and thus improve reaction kinetics. Two major advantages of plasma incorporation are the reduction of both reaction time and temperature. The PECVD can be 10 times faster than traditional CVD[57]. 

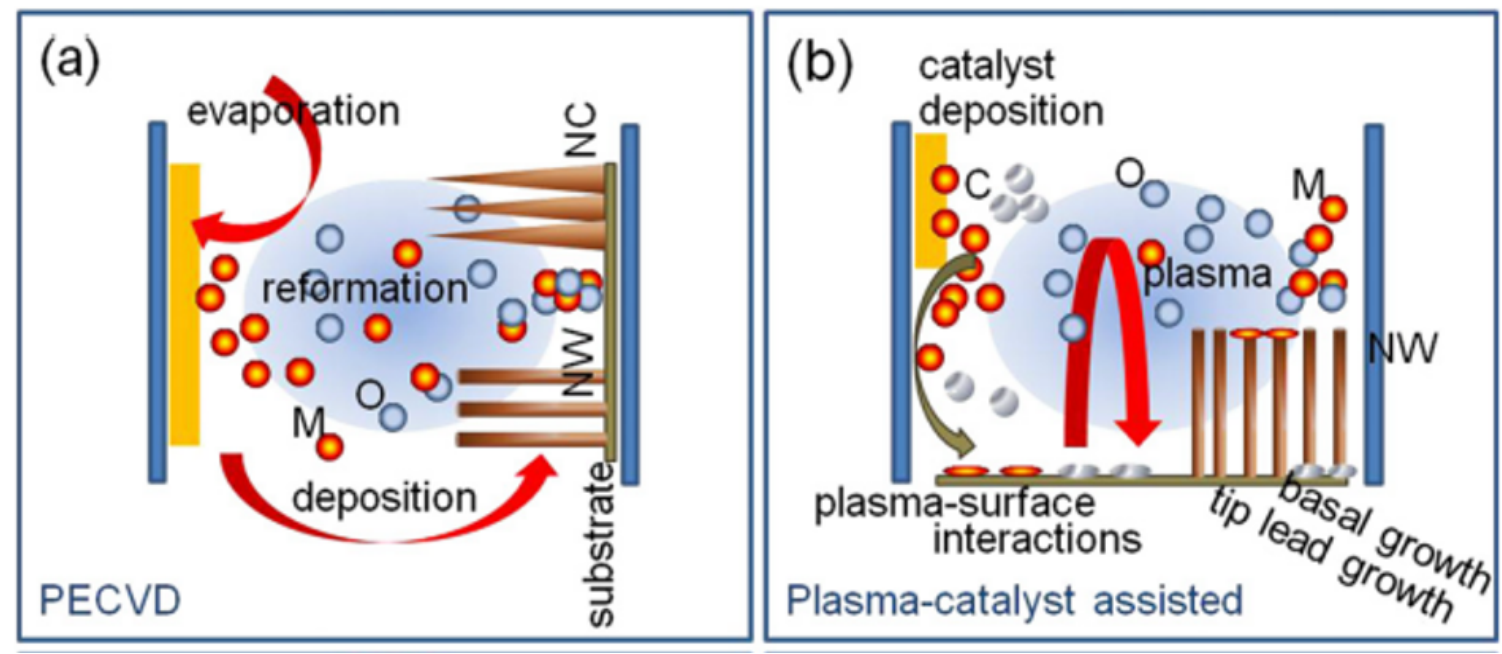

Figure 2.4.3: Schematic of PECVD without using catalyst (a) and with using catalyst (b). Adapted from Ref. [57]

For example, zin oxide nanorods have been synthesized using PECVD [58]. The alignment and density of nanorods could be controlled by substrate type, electric field and catalyst layer. In another study by Sunkara's group[59], the synthesis of silicon nanowires took place at a very low temperature of $180{ }^{\circ} \mathrm{C}$ for $15-30$ minutes using $\mathrm{RF}$ PECVD. Typically, the temperature requirement for silicon nanowire synthesis using traditional CVD is about $600{ }^{\circ} \mathrm{C}[21]$

\subsubsection{Direct plasma synthesis}

Direct plasma synthesis is a new method for large scale, fast synthesis of 1D nanomaterials. The method involves direct exposure of precursor to plasma flame. The precursor may flight through the plasma flame or expose to plasma flame supported on a substrate or foil of the same materials as shown in Figure 2.4.4. This method commonly does not use any catalyst. Transformation of metal particles to metal oxide nanowires upon plasma exposure involves melting or vaporization followed by plasma interaction 
leading to reshaping into nanowires. The direct plasma synthesis is usually short residence time method which is in order of second to minutes.
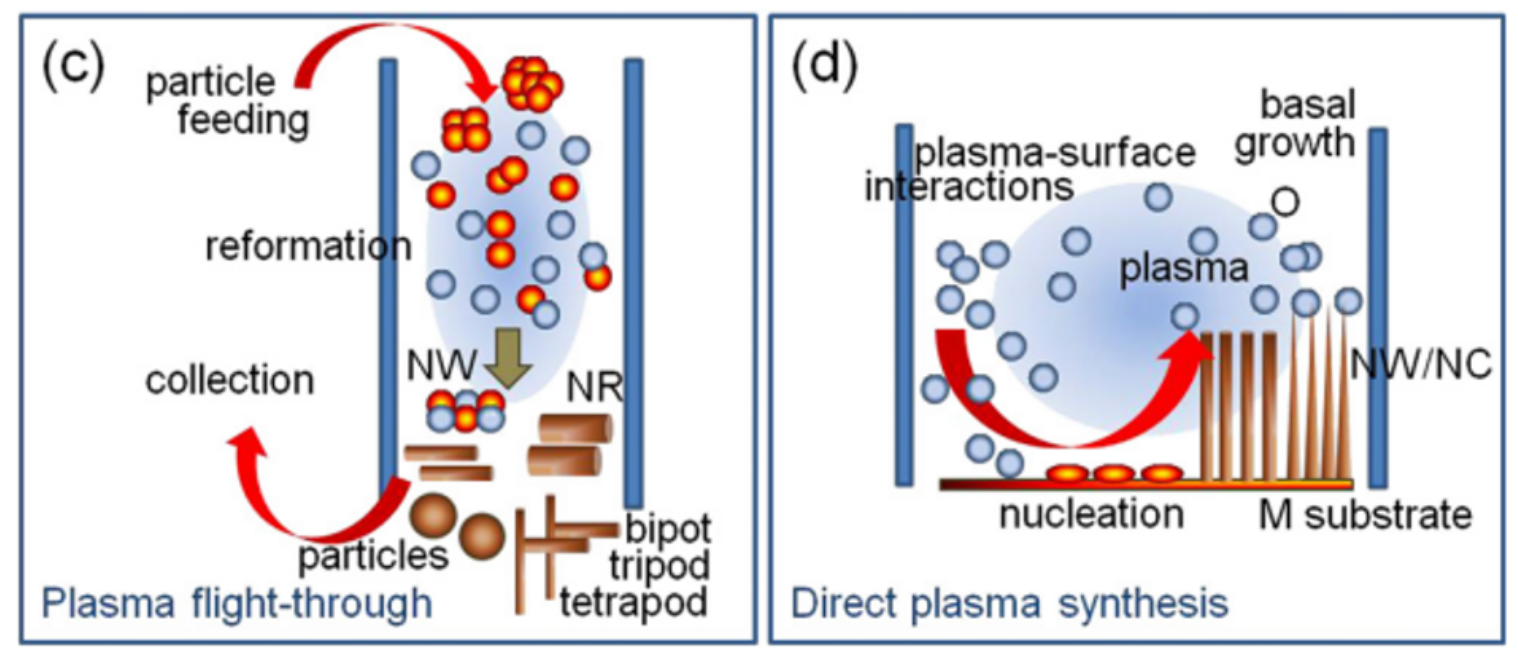

Figure 2.4.4: Schematic of direct plasma synthesis: (c) plasma flight-through and (d) direct contact plasma synthesis. Adapted from Ref. [57]

Direct plasma synthesis has been extensively used by Sunkara's group for a number of metal oxide systems such as zinc oxide, tin oxide, titania, alumina, niobium oxide, hematite, etc $[17,60,61]$.

In their study for zinc oxide, tin oxide, titania, alumina nanowires, the micron size metal powder of corresponding oxide is pour directly into atmospheric microwave plasma of 0.5 to $2 \mathrm{~kW}$ using mixture of oxygen and nitrogen or air. Variables such as metal powder size, plasma power, gas composition are used to control the morphology of nanowires and yield. The resulting nanowires of zinc oxide are about $30-300 \mathrm{~nm}$ in diameter, and 1-10 microns in length. It has been shown that the production capacity of zinc oxide nanowire powders can be as high as $15-30$ gram per batch of 1.5 hours. In their study of niobium oxide and hematite nanowires, niobium and iron metal foil are 
exposed directly to RF ICP source. The oxygen species such as oxygen radicals and oxygen ions played an important role in the formation of single crystalline hematite nanowires.

A DC plasma discharge was also used to prepare zinc oxide at $70 \mathrm{~kW}$ plasma powder and at atmospheric pressure[62]. Nitrogen is used as carrier gas to entrain zinc metal powder into the plasma jet. They found that the use of high ratio of argon in carrier gas favor the formation of nanorod and nanowire-like structures. A production rate of 1.2 kg per hour has been reported. However, there was large portion of nanoparticles in the product.

Peng et al. [63] reported a study on the synthesis of zinc oxide nanorods. A high RF plasma discharge at $30 \mathrm{~kW}$ was used along with argon and nitrogen. Powders of zinc, zinc oxide, and zinc carbonate were used as starting materials. The starting materials were vaporized, oxidized and transformed into nanorods upon plasma exposure. The nanorod formation was influenced significantly by supersaturation of zinc vapor and oxygen partial pressure. The production capacity of 24 grams/min has obtained using oxygen flow rate and zinc powder feedstock of $5.0 \mathrm{~L} / \mathrm{min}$ and 20 grams $/ \mathrm{min}$, respectively.

In summary, plasma synthesis has been shown to reduce the reaction time scale and to increase the production rate. However, only direct plasma synthesis could produce up to 10 grams/min while the PECVD could produce much smaller amount of nanowires (see Figure 2.4.5) 


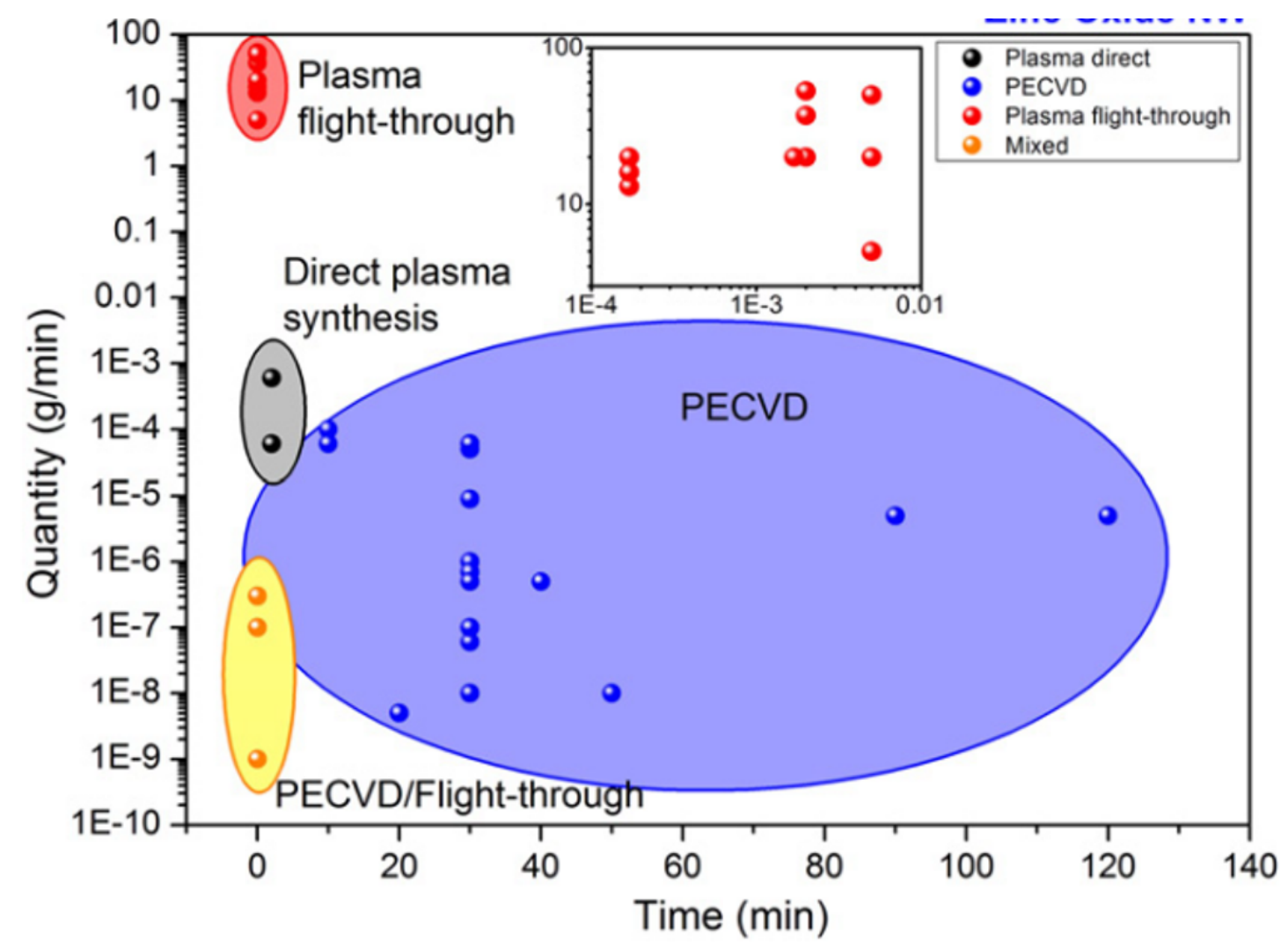

Figure 2.4.5: Nanowire production rate for zinc oxide nanowires by different plasma based technique. Adapted from Ref. [57]

\subsection{Hydrocarbon flame based techniques}

Flame based techniques or aerosol flame syntheses are powerful single-step synthesis techniques for nanomaterials. The aerosol flame syntheses are continuous and fast methods for producing nanomaterials in gas phase. The production of several nanomaterials such as titania, alumina and fumed silica has been commercially available[19, 64]. In aerosol flame synthesis, vapors or droplets of metal precursors are combusted in flame and are converted to metal oxides in two mechanisms as shown in Figure 2.5.1: (1) gas-to-particle and (2) droplet-to-particle. Gas-to-particle route usually produces small, dense particles while droplet-to-particle results in larger, porous particles. Aerosol flame synthesis can be divided into three categories based on the 
precursor used: vapor-fed aerosol flame synthesis (VAFS), flame spray pyrolysis (FSP) and flame-assisted spray pyrolysis (FASP) [65].

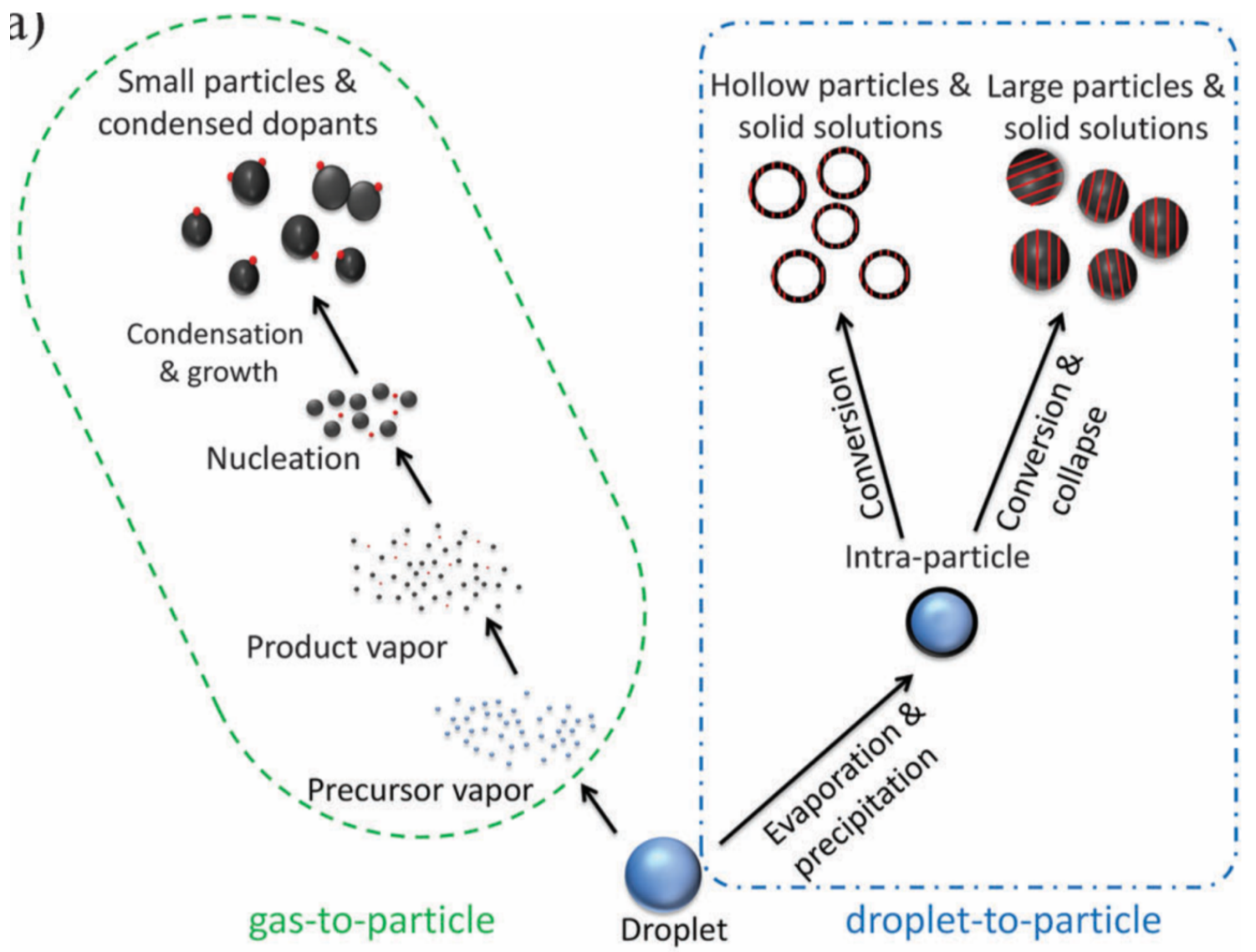

Figure 2.5.1: Two possible mechanisms proposed for particle formation in flame synthesis. Adapted from ref. [66]

There are typically two types of flame configurations for aerosol flame syntheses: premixed flame and diffusion flame reactors. The premixed fuel and oxygen is used in the premixed flame which provides more uniform temperature profiles and less soot formation by controlling the ratio of fuel to oxygen. The diffusion flame, however, is safer operation because no flashback issue. The diffusion flames are commonly used for manufacturing of ceramic powder in the industry. Three common fuels are natural gas, propane, and acetylene. Natural gas is the cheapest one but gives lowest temperature 
among three. Oxy-acetylene flame is the most expensive but gives highest temperature up to $3100{ }^{\circ} \mathrm{C}$. The variables are precursor state (liquid or vapor phase), precursor concentration, precursor feed rate, flame configuration, quenching rate, etc. Aerosol flame synthesis are primarily used for spherical nanomaterial production. However, 1D nanomaterials can be produced via aerosol flame synthesis such as zinc oxide nanorods.

\subsubsection{Vapor-fed aerosol flame synthesis (VAFS)}

VAFS has been widely used for production of ceramic powder such as titania, fumed silica, and alumina [19]. The VAFS requires a precursor which is easily evaporated such as chlorides $\left(\mathrm{TiCl}_{4}, \mathrm{SiCl}_{4}, \mathrm{AlCl}_{3}\right)$. The precursors are converted to metal oxide particles via gas-to-particle mechanism as shown in Figure 2.5.1. Typically,

precursors are fed into flame as vapors. Vapors are then oxidized in the flame to form oxide nuclei. The nucleation followed by coalescence, aggregation and coagulation to form nanoparticles. Gas-to-particle commonly produces small and dense nanoparticle agglomerates. A large number of oxides have been synthesized using VAFS such as titania, silica, alumina, vanadium oxide and tin oxide particles[19]. However, due to volatile precursor requirement, no study has been shown for the synthesis of zinc or tin oxide nanowires/nanorods.

\subsubsection{Flame-assisted spray pyrolysis (FASP)}

When a non-combustible liquid precursor is used in aerosol flame syntheses, it refers as flame-assisted spray pyrolysis. Nitrate salt solution is commonly used as precursor. The precursor solution is sprayed as liquid droplets into flame such as hydrocarbon flame where metal salt is converted to metal oxide via droplet-to-particle 
mechanism as in Figure 2.5.1. Solvent evaporates from liquid droplets creating hollow structure particles.

FASP is a common method for producing hollow spherical particles or larger spherical particles than VAFS[67]. In a special study done by Hembram et al. [68], zinc oxide nanorods were prepared using zinc nitrate as precursor by FASP. The nanorod formation was believed to differ from liquid-to-particle mechanism. Accordingly, dissociation of zinc oxide occurs at high temperature forming zinc vapors. Zinc vapors get re-oxidized to form zinc oxides acting as seeds for nanorod growth by vapor condensation. The production rate was reported to be $3 \mathrm{~kg}$ per hour.

\subsubsection{Flame spray pyrolysis (FSP)}

FSP is the most common method for nanomaterial production at large scale. The combustible precursors such as organic based metal precursor are primarily used in FSP. In FSP, combustible precursor solution evaporates and get combusted in the flame. FSP usually produces more homogeneous nanoparticles than the previous flame based methods due to the use of combustible precursor.

There have been extensive studies on spherical nanoparticle synthesis using FSP by Pratsinis's group in Switzerland. In most cases, spherical particles were obtained. For example, zinc oxide $(10 \mathrm{~g} / \mathrm{hr})[69]$, tin oxide $(36 \mathrm{~g} / \mathrm{hr})[70]$, silica $(9 \mathrm{~g} / \mathrm{hr})[71]$, and zirconia(100-500 g/hr) [72] nanoparticles have been produced by FSP. Zinc acrylate in methanol, tin (II) 2-ethylhexanoic acid in ethanol, hexamethyldisiloxane in ethanol, and zirconium 2-ethylhexanoate were used as precursor solution, respectively.

1D nanomaterials such as carbon nanotubes have been synthesized using flame 
[73]. However, controlled synthesis of such as 1D inorganic materials in flame is difficult. Rod-like particles have been obtained in flames but only at low content, and the formation of such rod-like particles is not controlled[69]. Height et al.[74] has shown that the use of dopants in FSP of zinc oxide favors formation of zinc oxide nanorods. Nanorod formation was due to the higher valency and coordination of tin and indium dopants relative to zinc. It is worth noting that zinc vapor formation in flame synthesis is an important factor for the 1D growth in flame synthesis[68]. For example, diethyl zinc precursor decomposed into zinc vapor in the synthesis of zinc oxide nanorods using FSP. The zinc vapors were believed to react with water vapor to form either zinc hydroxide or zinc oxide. The recrystallization of zinc oxide would then be the mechanism for nanorod formation[75]

\subsection{Summary}

Gas phase synthesis techniques, including CVD, plasma-based techniques and flame-based techniques, are shown to potentially produce large quantities of 1D nanomaterials due to their shorter residence time. The resulting dry product eliminates the need for post-processing steps, which make them scalable easily. However, CVD or PECVD requires a substrate, metal catalyst, vacuum processing pressure that make them unsuitable for scalable manufacturing method. Direct plasma synthesis, on the other hand, offers a number of factors that make them viable for large scale production such as continuous process, substrate and catalyst-free, fast time scale (Figure 2.5.2) and atmospheric pressure. 


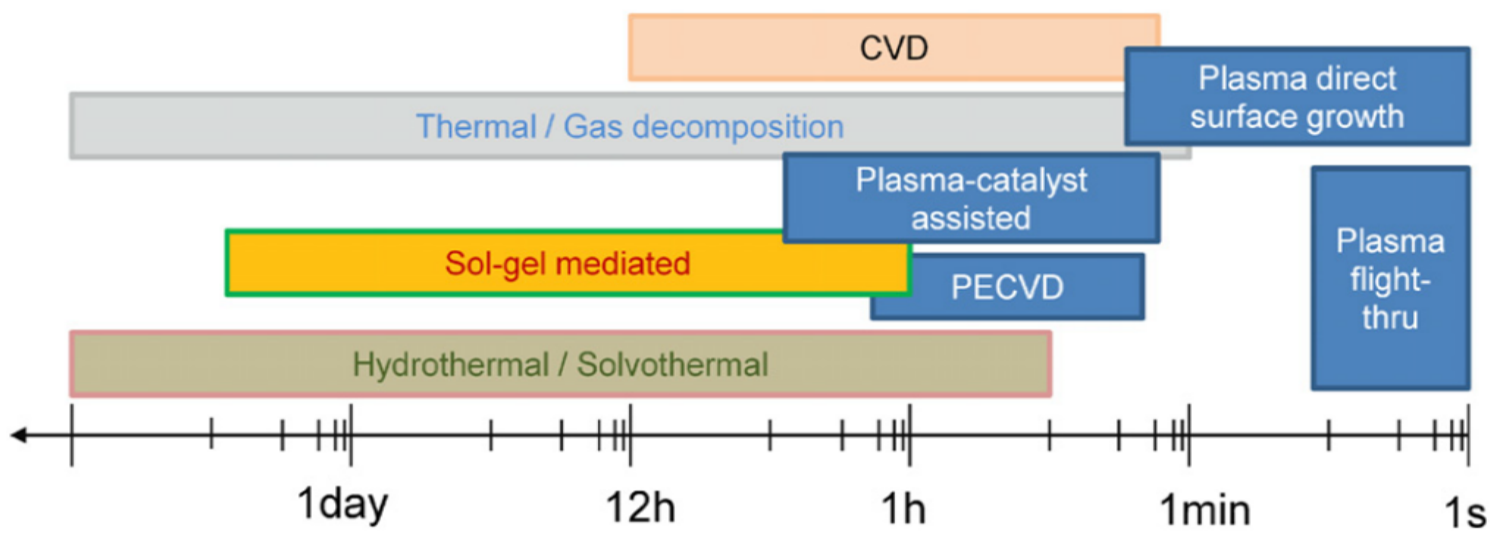

Figure 2.5.2: Time scale requires for different synthesis techniques. Direct plasma synthesis offer shortest time scale compared to all other methods. Adapted from Ref. [57]

Flame-based techniques are obviously viable routes for mass production of nanomaterials. However, the current state of the art flame-based techniques are still limited to produce only zinc oxide nanorods using non-common, expensive precursor such as diethyl zinc or foreign metals as dopants. In such techniques, the nanowire/nanorod production using flame-based methods is not well understood yet. Combination of direct plasma and flame based techniques seems to be an ideal method for bulk production of 1D materials. In this dissertation, a large scale production of metal oxides using direct plasma, hydrocarbon flame-based or combination of plasma and hydrocarbon flame is explored. These methods can potentially produce nanowires/nanorods at kilogram to ten kilograms per day using a new design fluidized bed reactor and cheap starting materials. 


\section{CHAPTER III}

\section{EXPERIMENTAL}

\subsection{Introduction}

This chapter describes the detail on reactor design, synthesis and characterization methods used in this dissertation. The reactor design includes the modification of the downstream atmospheric microwave plasma reactor and design of a fluidized bed reactor. The custom-designed reactors include: (1) a downstream atmospheric, microwave plasma and fluidized bed reactors for direct oxidation of metal powders; (2) upstream atmospheric microwave plasma reactor for oxidation of metals/metal oxides in presence of alkali compounds; and (3) a CVD reactor for reactive transport studies. Various methods were used for characterizing the resulting nanowire powders for their optical, structural, catalysis, and electrochemical properties.

\subsection{The downstream atmospheric microwave plasma reactor}

In one of our efforts to scale up the production of nanowire materials, a downstream atmospheric microwave plasma reactor has been designed[17, 76]. Briefly, the reactor consists of two main components: (1) the microwave plasma system; (2) the vertical tubing reactor.

The microwave plasma system includes standard components such as a 
magnetron, a circulator, a dummy load, a power detector, an impedance matching system, and a custom-design applicator (Figure 3.2.1). All the components are in communication through a rectangular waveguide WR284. The magnetron generates microwaves of 2.45 $\mathrm{GHz}$ into the waveguide. The circulator connected to water cooled dummy load only allows the propagation in one direction and is used to prevent the reflected microwave from entering the magnetron. The power detector measures the forward and reflected powder. The impedance matching system comprises of a circuit of resistors and capacitors matches the impedance of the source (magnetron) and the load (plasma) for maximum power transmission and to reduce the reflected power to zero. The applicator is designed in such a way that the microwave is concentrated at the center of a quartz tube inserted in applicator. Argon is introduced into the applicator at an angle to form downward helical flow. The helical flow is used to prevent the heating on quartz tube causing by plasma flame. A copper rod is used to ignite the plasma and is removed quickly after ignition of plasma. The plasma flame is maintained by introducing gases such as air, or mixtures of gases such as argon, oxygen, nitrogen, or hydrogen, etc.

Plasma flame is generated in downward direction inside a vertical quartz tube that is used as the reactor chamber. A vacuum pump is used to keep the plasma flame in downward direction. Metal powder such as zinc powder is poured from the top of the quartz tube at the applicator. Zinc metal powder falls into plasma flame and reacts with oxygen radicals to form zinc oxide nanowires. The product is collected in a collecting cup at the bottom of reactor tube. The reactor has been shown to produce 15-20 grams in 15-20 minutes a batch. However, the conversion was low of about $20 \%$. An additional step of purification by dispersing the product into alcohol solution followed by drying 
step needed to get higher quality nanowire powders. Eventually, the production rate was about 5 grams per hour. There were several issues associated with the reactor that make it limited in producing large amount of metal oxide nanowire powder at high conversion rate. The issues were feeding method, collecting method, heating, and ozone generation.

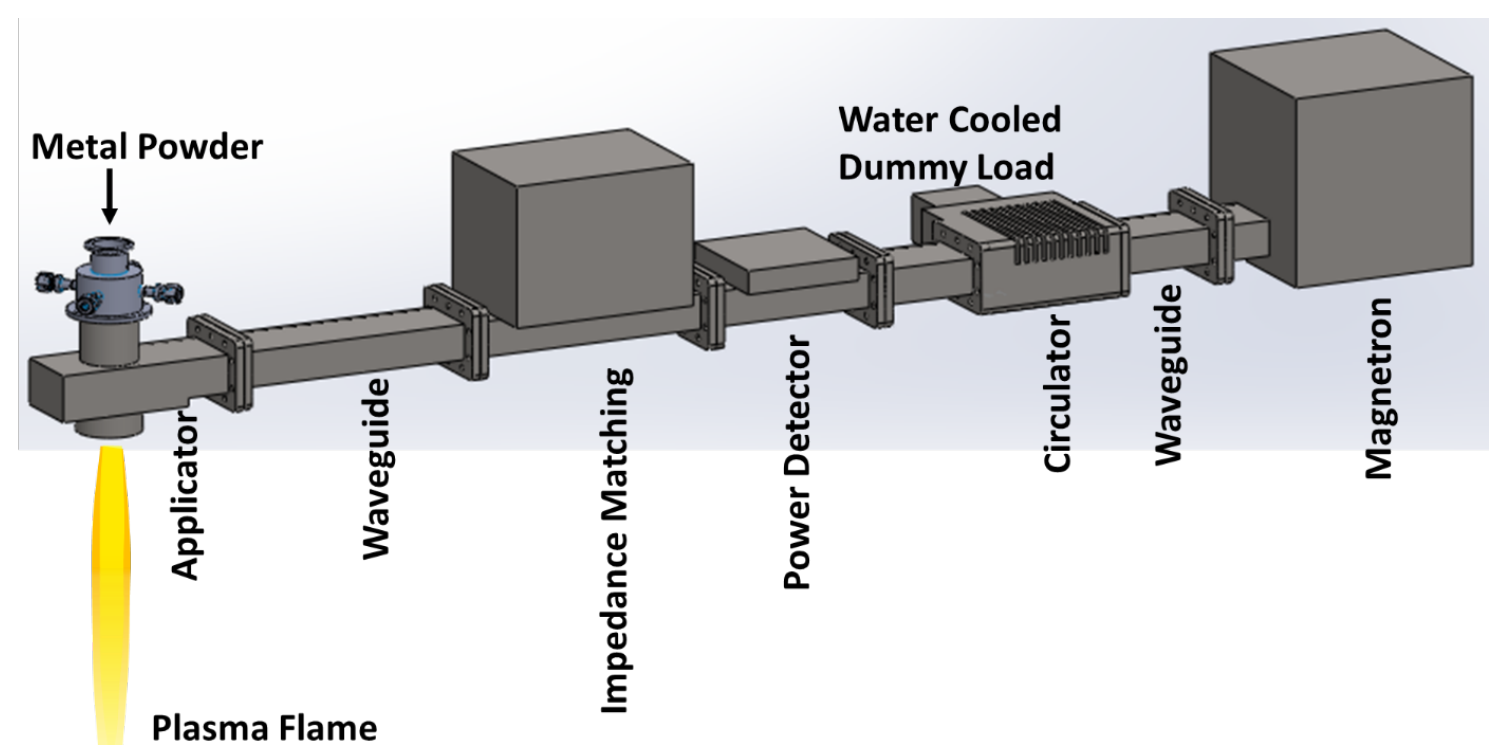

Figure 3.2.1: Schematic of the downstream atmospheric microwave plasma system

\subsubsection{Challenges with the downstream atmospheric microwave plasma reactor}

The first issue was the feeding method. Typically, the metal powder was picked using a scoop and poured into the reactor from the top as shown in Figure 3.2.2. The metal powder fell down into the plasma zone under the gravity as big clusters. It was also very difficult to control the feeding rate of metal powder. As a result, the metal particle dispersion was low and most of the particles were not exposed to the plasma. Since the metal particles were moving very fast so that there was only reaction at particles on the surface of the big clusters. The unreacted metal particles inside the big cluster contributed 
to the overall low conversion of metal particles to metal oxide nanowires.

The second issue was the collecting method. As shown in Figure 3.2.2, a collecting cup was placed at the lower end of quartz tube before the vacuum pump. As we knew that the density of metal particles is much higher than that of metal oxide. The metal particles tended to fall down very fast directly to the collecting cup. However, the lighter density oxide particles tended to be fluidizing and following the gas flow to the vacuum pump. Consequently, there were lot of big metal clusters and unreacted metal particles collected in the collecting cup. Observation showed that a lot of metal oxide particles went to vacuum pump. This was the reason why the vacuum pump got clogged up at suction chamber.

The other issues included heating, and ozone generation. The heating resulted in the deposition of metal oxides along the piping which reduced the conversion efficiency and increased the maintenance time. Also, the use of microwave plasma generated significant amount of ozone.

In summary, all of the issues are needed to be addressed in order to increase the conversion efficiency, production rate as well as to meet the safety standard. 


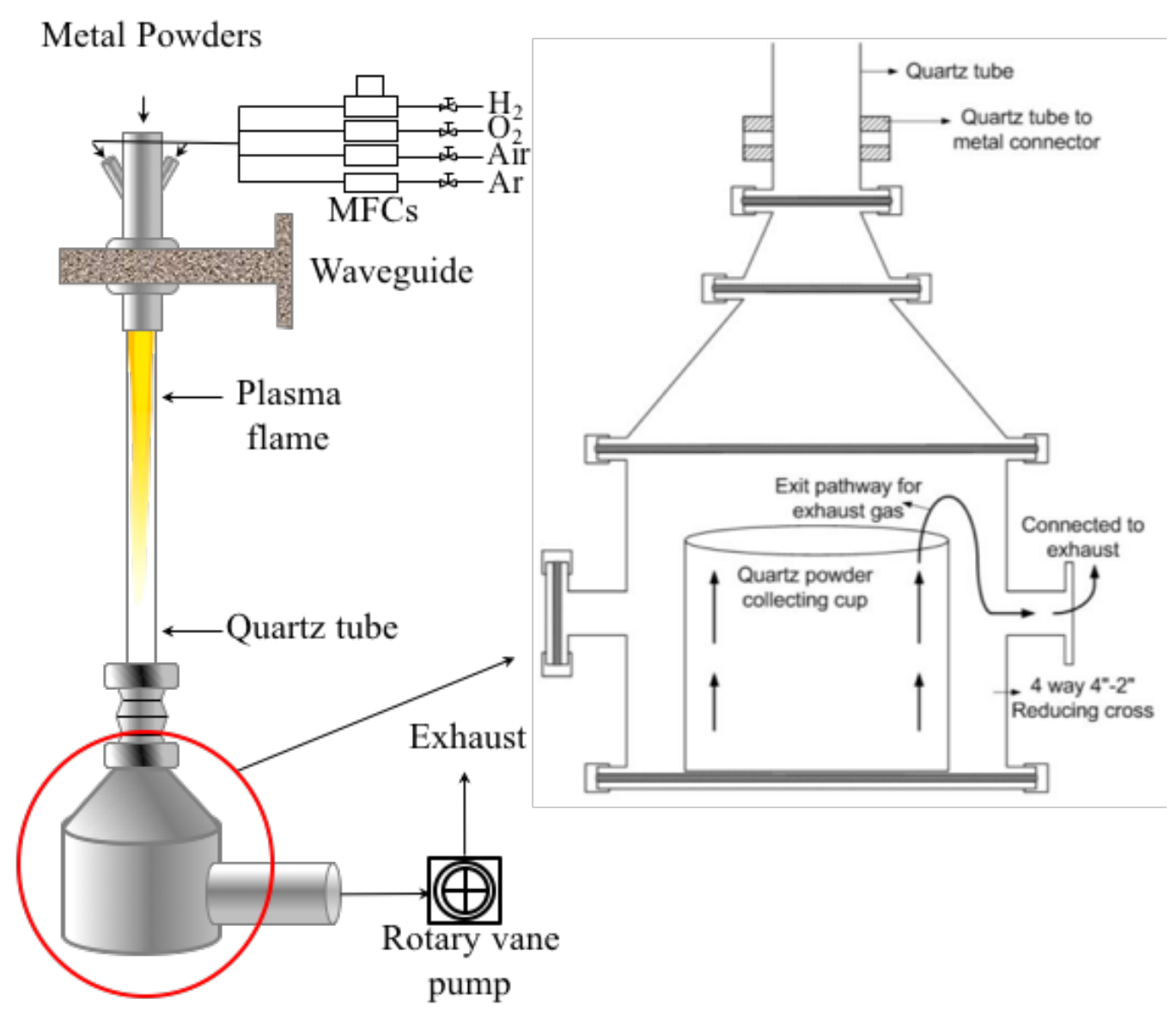

Figure 3.2.2: Schematic of the Downstream Atmospheric Microwave Plasma Reactor

\subsubsection{Reactor design modification and results}

As described in previous section, the issues should be addressed to increase the yield and to meet safety standard. In order to solve the problem with feeding, a semiautomatic powder was designed using a metal funnel with a vibrator attached on it. A metal mesh was used to control the dispersion (Figure 3.2.3). The mesh size could be changed to adjust the feeding rate. The feeder was working great for at least 5 minutes before metal powder needed to fill into the top funnel. The feeder also eliminated a need to stand nearby the reactor all the time to manually pour metal powder into the reactor. 

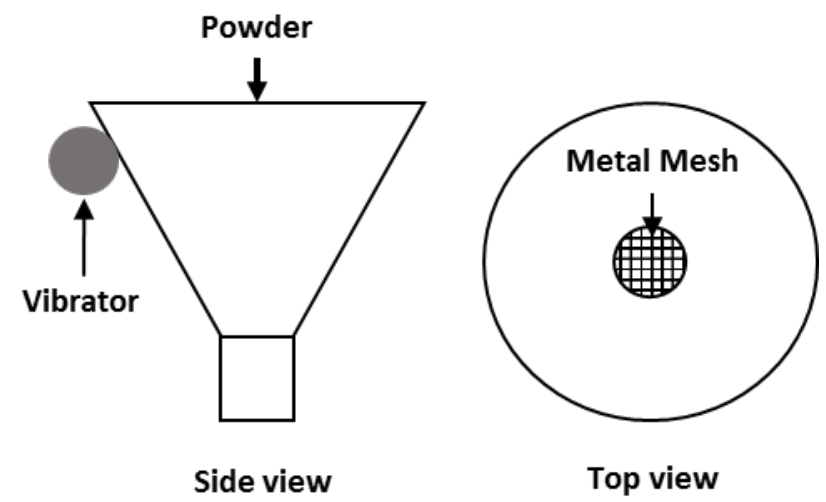

Figure 3.2.3: Schematic of the semi-automatic powder feeder

A filter housing was also designed and was placed before the vacuum pump as shown in Figure 3.2.4. A F1 filter unit enclosed in the metal housing was used to collect metal oxide powder coming from the reactor and to prevent the powder from entering the vacuum pump. A set of copper cooling coils were wrapped on the piping and filter housing to minimize the deposition. A hot surface was reported to increase the deposition rate of metal oxides [77]. A carbon filter was also place at the outlet of the pump to remove ozone generated by plasma. The air source was also switched to compressed air which is available for free to increase the nanowire production economic. Figure 3.2.4 showed the overall schematic of the reactor and couple pictures after modification. 

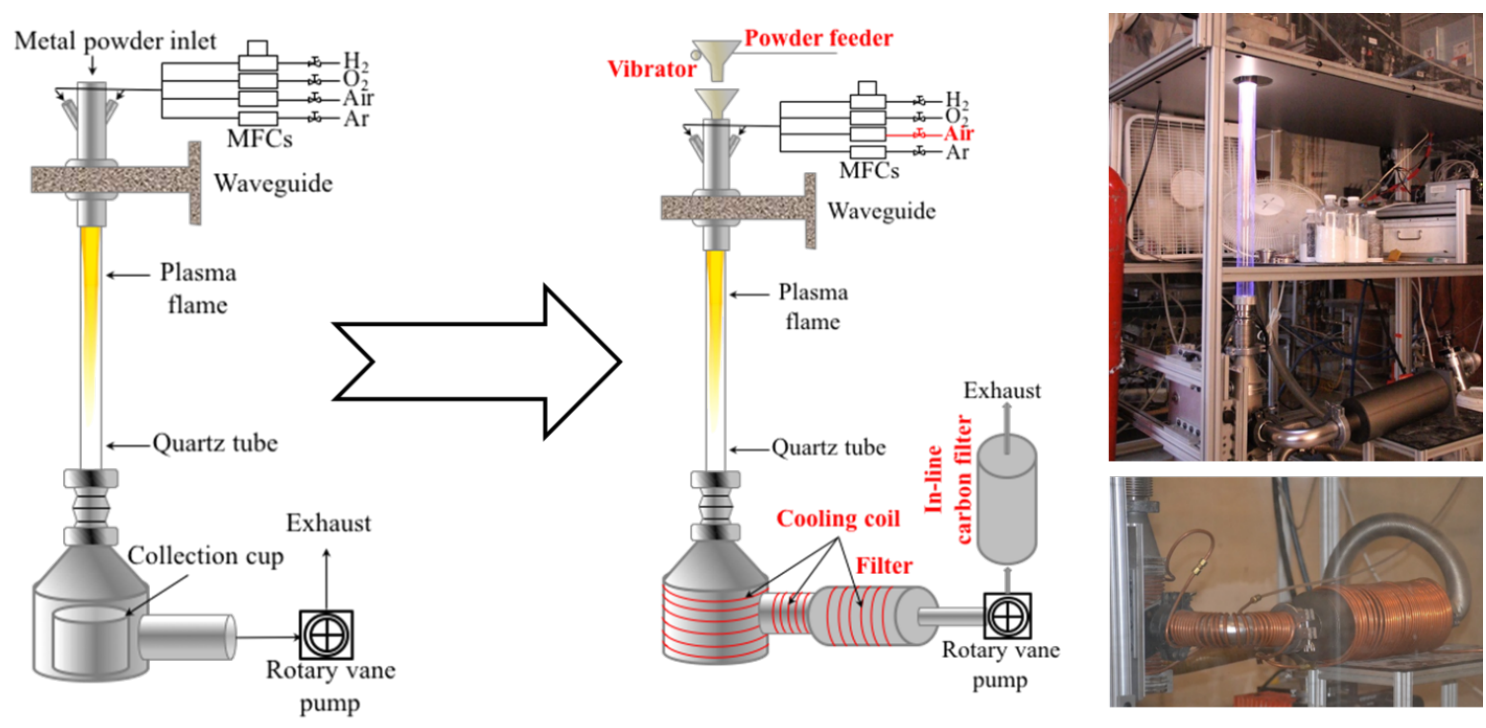

Figure 3.2.4: Schematic of the modified reactor and photos of plasma column, filter, cooling coil

Metal powder dispersion has been shown to be an issue for conversion and productivity [78]. The new semi-automatic powder with metal mesh helped increasing the metal dispersion. Highly dispersed metal particles increased exposure of metal particles to plasma flame. As a result, significantly less clustered unreacted metal particles were collected at the bottom of the reactor. The conversion has been increased up to $90 \%$. The powder collected in the filter housing was $100 \%$ zinc oxide (see the XRD pattern, and color of the powder in the bottle in Figure 3.2.5). No peak attributed to zinc metal was observed. The SEM images also showed production of good quality nanowires using the new powder feeder. The image showed similar nanowire morphology to that of previous work. However, the previous work required an additional step of purification [17]. The most important thing was that the production capacity has been increased up to 20 grams per batch. The operating time was also increased up to 1.5 hours. We also observed much less powder deposited on inner wall of piping and filter housing which 
confirmed the effective of copper cooling coil.
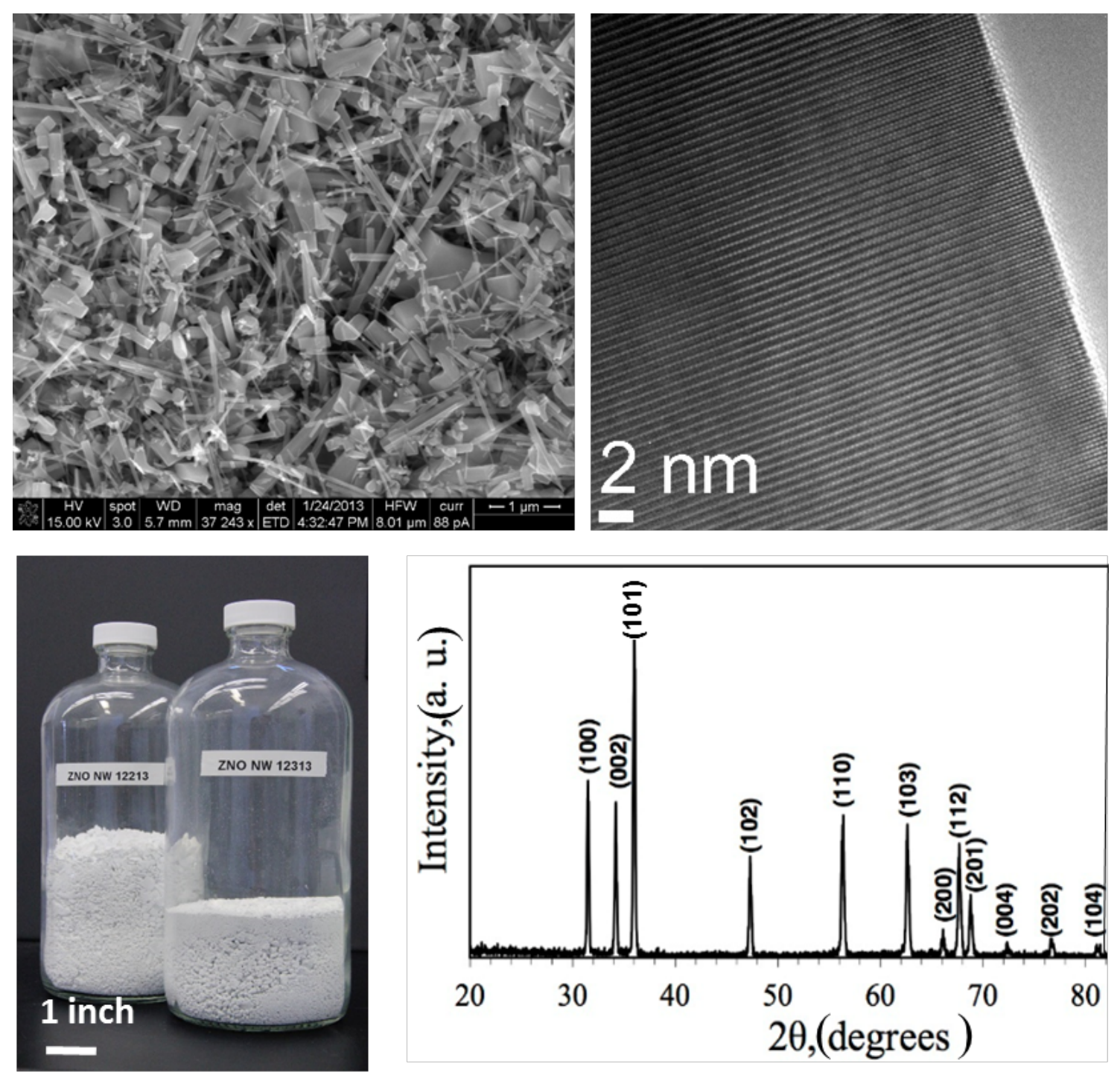

Figure 3.2.5: $\mathrm{SEM}$ image of $\mathrm{ZnO}$ nanowires (top left), $\mathrm{TEM}$ of $\mathrm{ZnO}$ nanowires (top right), Bottles of $\mathrm{ZnO}$ nanowire powder, $\mathrm{XRD}$ pattern of of $\mathrm{ZnO}$ nanowire power collected in filter housing.

\subsubsection{Challenges}

As mentioned in the previous section, the downstream atmospheric microwave plasma reactor has been shown to produce up to 20 grams per batch or 50-100 grams a 
day. However, that still did not meet the goal for bulk production. The goal of this work is to produce up to several kilograms of nanowire powder per day. Typically, there were two options to increase the production capacity: (1) is to increase the feeding rate of metal powder; (2) is to increase the operating time. However, persistent problems were observed with the high feeding rate or long operating time.

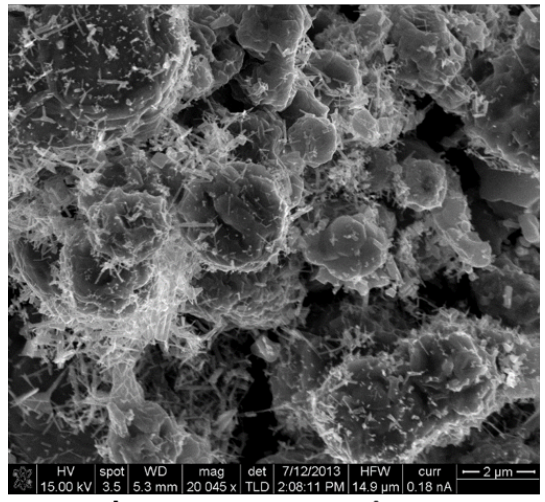

Low conversion

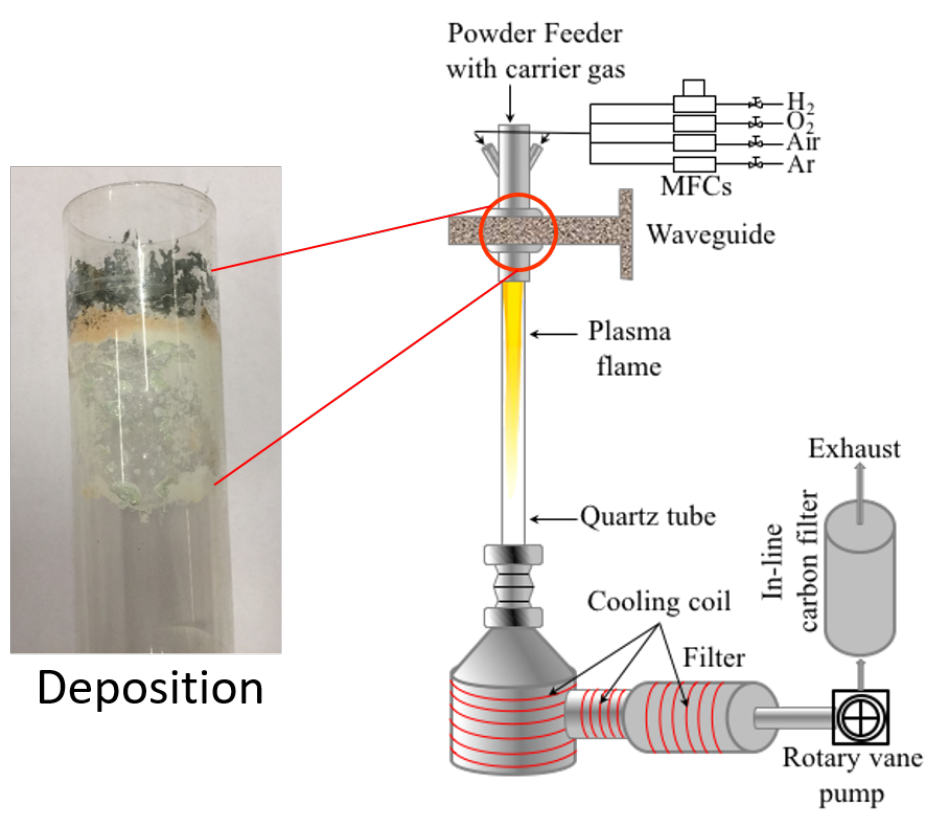

Figure 3.2.6: SEM image of powder collected in the Filter at 5 grams per minute; a photo showing deposition on quartz tube and schematic of the downstream atmospheric microwave plasma reactor

In order to increase the feeding rate, a powder feeder was used along with argon as carrier gas. The use of carrier gas made the metal particles move too fast along the quartz tube. This resulted in lot of unreacted metal particles in the collecting filter as shown in a SEM image in Figure 3.2.6 or the conversion was very low of about 10-20\%. It turned out that the use of powder feeder with carrier gas has lowered the residence time from 0.43 seconds to 0.01 seconds due to drag force of carrier gas flow exerted on the 
metal particle. The residence time of 0.01 seconds was too short for the reaction to be completed. The residence time was calculated using reactor length of 30 inches, total gas flow rate of 30 LPM as following: (equation 3.2.1: 6-227 Perry's Handbook)

$$
F_{\text {total }}=F_{\text {buoyancy }}+F_{\text {drag }}=\frac{1}{6} \pi d_{p}^{3} \rho g+\frac{\pi}{8} C_{D} \rho d_{p}^{2} u_{\infty}^{2}
$$

where $\mathrm{F}_{\text {total }} \quad$ : total force exerted on the particle, $\mathrm{N}$

$$
\begin{array}{ll}
\mathrm{F}_{\text {buoyancy }} & : \text { buoyancy force, } \mathrm{N} \\
\mathrm{F}_{\mathrm{drag}} & : \text { drag force due to flow, } \mathrm{N} \\
\mathrm{d}_{\mathrm{p}} & : \text { particle size, } \mathrm{m} \\
\rho & : \text { particle density, } \mathrm{kg} / \mathrm{m}^{3} \\
\mathrm{C}_{\mathrm{D}} & : \text { drag coefficient } \\
\mathrm{u}_{\propto} & : \text { fluid velocity, } \mathrm{m} / \mathrm{s}
\end{array}
$$

Moreover, when the operating time was increased, there was persistent deposition built up on the inner wall of quartz tube. The deposited film became thicker and thicker overtime. The resulting films started to absorb microwaves with increasing intensity. As a result, the quartz tube heats up and melts causing the plasma flame to be unstable. Experiments have shown that the plasma became unstable after about one and a half hours. A clean up or tube exchange was needed for later operation.

In summary, in order to obtain several kilograms of nanowire, a better reactor design is needed. The following section will discuss about designing, optimizing, and operating of our new fluidized bed reactor. 


\subsection{Design of a fluidized bed reactor for mass production of metal oxide nanowires}

\subsubsection{Introduction}

Among reactors used in gas phase syntheses, chamber-type reactor usually requires the evaporation of precursor or the use of a substrate in vacuum condition which limited in scalability[79]. Tubular reactor in which the precursor flight through, on the other hand, has been proven to allow continuous processing [71]. However, it is difficult to control the residence time which contributes directly to low conversion[17]. In catalyst application, fluidized beds have been employed commercially for many years. The fluidized beds have been shown to increase heat and mass transfer significantly[80].

The new reactor would involve the use of either an atmospheric microwave plasma source or hydrocarbon flames for rapid oxidation of metal powder to metal oxide nanowires[81]. The fluidization would increase the residence of metal particles. Hence, the choice of heating source would not be limited to plasma source. A hydrocarbon flame, which is commercially used for nanoparticle production, can also be investigated $[72]$

\subsubsection{Reactor set-up}

The reactor is designed to deliver highly dense microwave jet of $500 \mathrm{~W}$ to $3 \mathrm{~kW}$ or high capacity hydrocarbon flame up to 15,000 BTU. The reactor system includes three main parts: the atmospheric microwave plasma system or hydrocarbon burner, the fluidized bed reactor body, the collecting system as shown in Figure 3.3.1. 


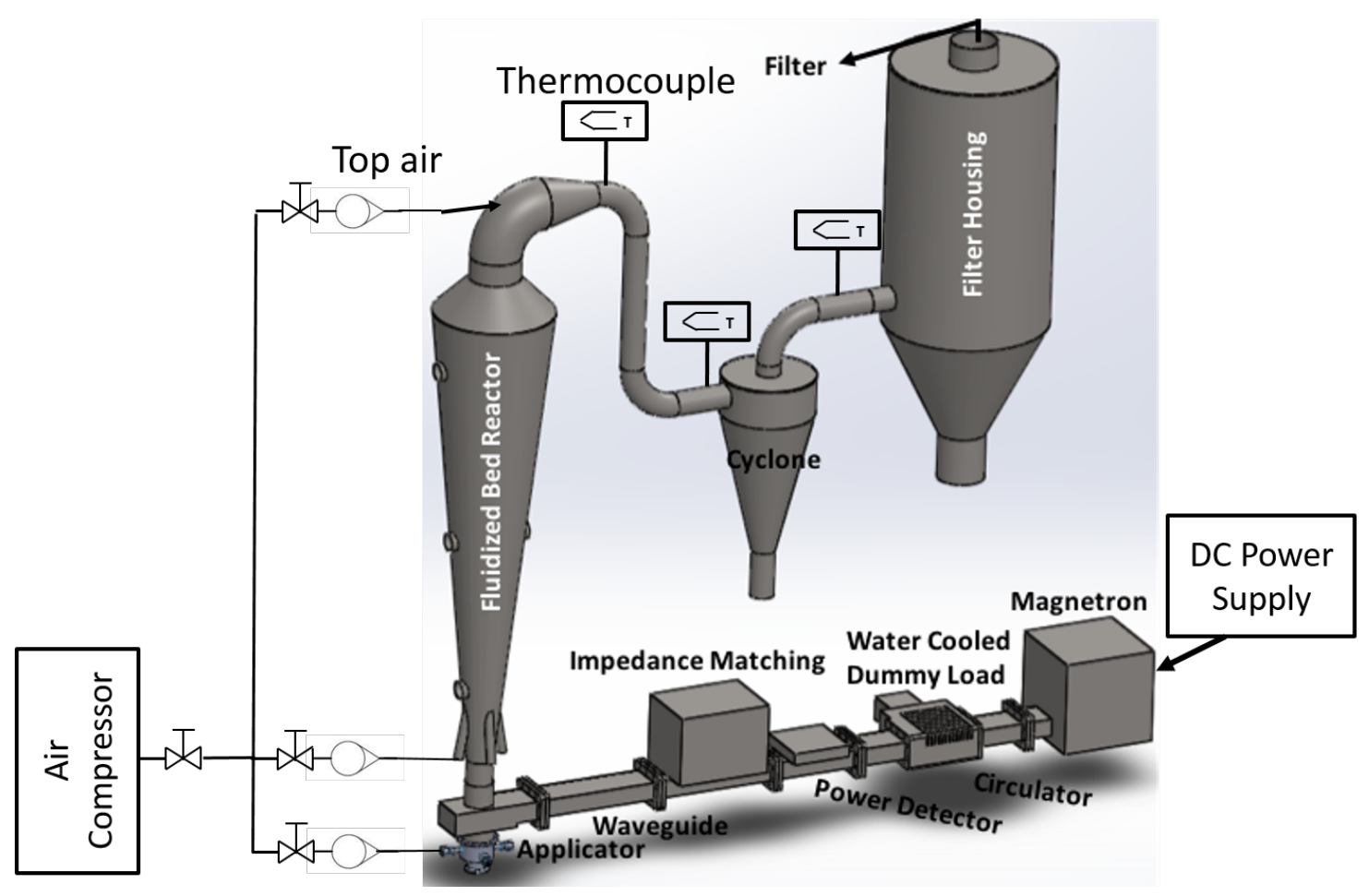

Figure 3.3.1: Schematic of fluidized bed reactor system with an atmospheric microwave plasma system

As shown in Figure 3.3.2, the reactor body is an upward conical 316 stainless steel column with the narrow end at the bottom. The reactor body is comprised of a top unit and a bottom unit. The bottom unit welded with a $50 \mathrm{~mm}$ Flange is to match with the $50 \mathrm{~mm}$ applicator of the plasma system or is used to match with the hydrocarbon burner. There are four inlets of $10 \mathrm{~mm}$ Swagelok tubing for either powder inlet or air dispersion inlet. The four viewports of $70 \mathrm{~mm}$ conflat flanges along the reactor body are used for viewing or coupling with thermocouples or top-feeding powder feeder. The top unit is joined to the bottom part by the 10" Flanges. The top unit is equipped with an elbow by a $100 \mathrm{~mm}$ Flanges as a connection. The reactor outlet at the elbow of $50 \mathrm{~mm}$ OD is connected with the collecting system at cyclone by a $50 \mathrm{~mm}$ OD piping system. The powder is delivered into the reactor using a powder feeder. The powder feeder is capable 
of delivering up to 15 grams of metal powder per minute. The two powder feeder units can be used to double feeding rate up to 30 grams metal powder per minute.

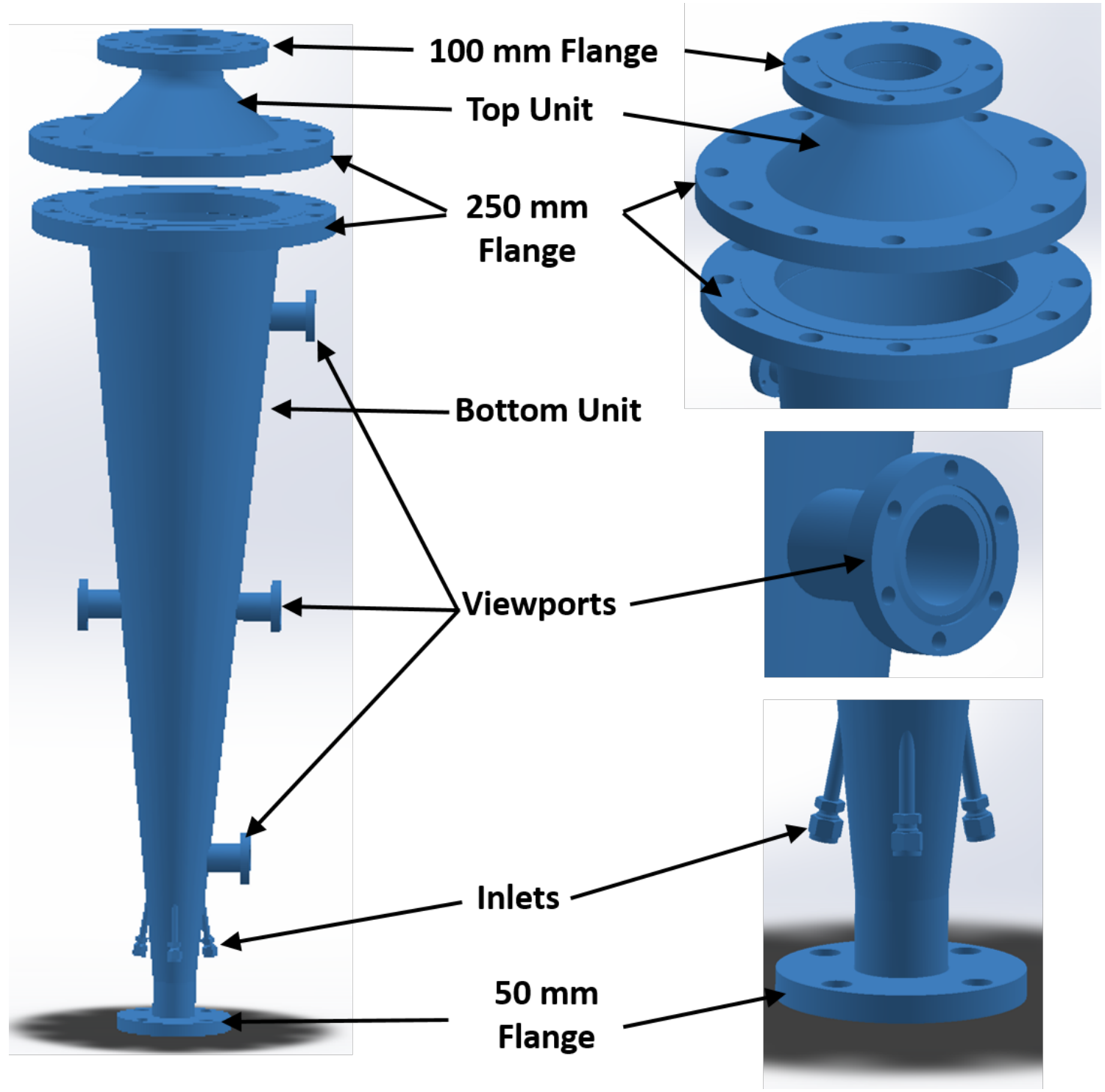

Figure 3.3.2: Schematic of fluidized bed reactor body

When plasma flame is employed, the atmospheric plasma system, just like the one described in the previous section 3.1, is used. The applicator is bolted to the $50 \mathrm{~mm}$ flange at the bottom of the reactor. Thus, the atmospheric microwave is generated into the reactor chamber. The plasma systems generated plasma flame into the fluidized bed 
reactor from the bottom using a plasma power of $1.5 \mathrm{~kW}$, a compressed air of 20 LPM. As shown in Figure 3.2.3, the upward vertical plasma flame is very stable as expected. However, the plasma length is about 12 inches which is too short for this fluidized bed reactor.

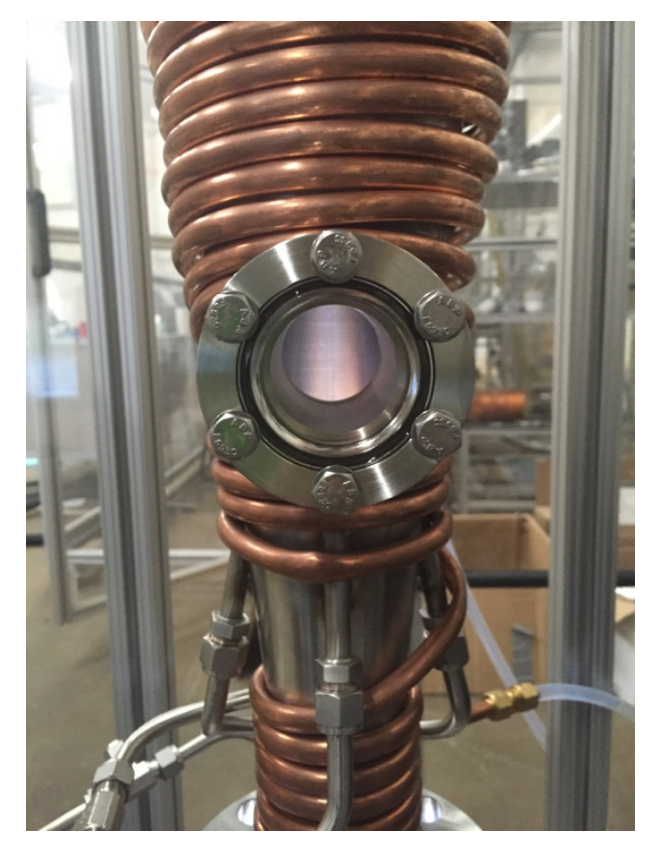

Figure 3.3.3: A photo showing plasma flame inside reactor chamber

When the reactor is operated using hydrocarbon, the microwave plasma system is replaced by a hydrocarbon burner. The hydrocarbon burner is custom-designed to work with an enclosed reactor chamber at slightly below atmospheric pressure. As shown in Figure 3.3.4, the burner includes a concentric tube of $20 \mathrm{~mm}$ for outer tube and $8 \mathrm{~mm}$ for inner tube in OD and $300 \mathrm{~mm}$ in length. The concentric tube is welded into a metal place which can be bolted to $50 \mathrm{~mm}$ flange at the bottom of the reactor body. Oxygen and hydrocarbon is supplied into concentric tube by two separate inlets. 


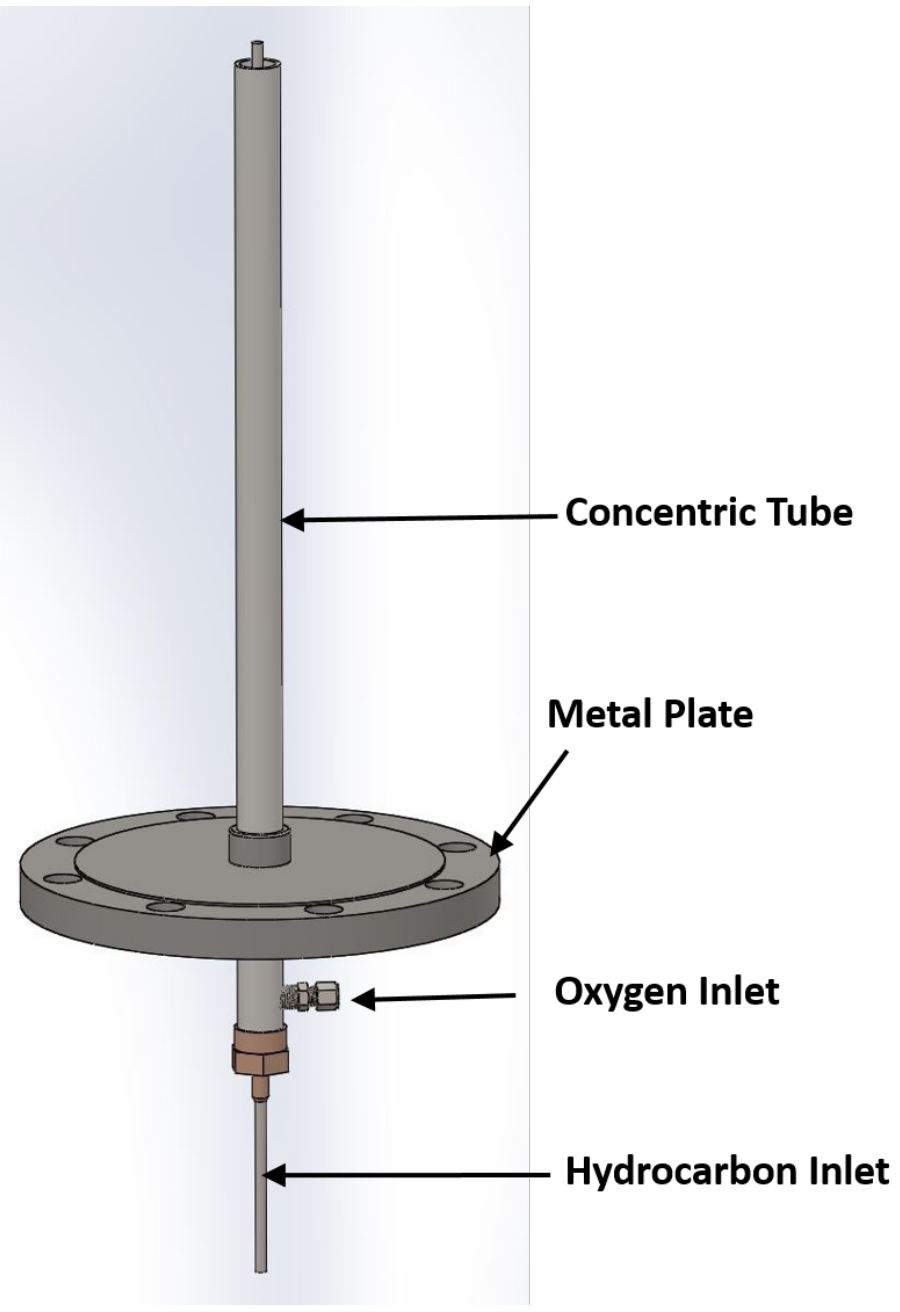

Figure 3.3.4: Schematic drawing of hydrocarbon burner

The collecting system consists of a high velocity cyclone, a filter housing and storage containers. The cyclone is designed to efficiently capture 20 to $99 \%$ of the powder coming from the reactor depending on the density, particle size of the powder and mass flow rate at the cyclone. There are four filter units at the filter housing. Each filter unit is equipped with a solenoid valve to efficiently remove powder from filter to storage container using air-pressure shock. The four filter units are used to make sure that the reactor could be operating continuously while still allowing collecting powder in three filter units and removing powder in the other filter unit. The $210 \mathrm{~L}$ storage 
containers allow to store up to $50 \mathrm{~kg}$ of nanowire powder. A carbon filter is setup at the outlet of filter housing to capture any powder leaking from filter housing and is used to remove the ozone generated by plasma flame. The outlet of carbon filter is connected to the ventilation.

An air compressor is used to supply air for plasma generation, metal dispersion and to control the efficiency of cyclone. Sets of thermocouples are used to monitor the temperature in different locations along the reactor system. Water cooling copper coil is used for reactor wall cooling.

\subsubsection{Reactor material, shape and dimensions}

One of the major issues with combustion reactor was corrosion due to long term exposure to oxidative gases at high temperature. Inconel was known as one the best corrosion resistance materials. However, Inconel was too expensive for industry applications. Therefore, Stainless Steel 316 was used for our reactor fabrication. The reactor wall thickness was $3 \mathrm{~mm}$ which meets the requirement for the oxidative environment[82].

The fluidized bed reactor was designed in such a way that there will be fluidization of particles with increased residence times. The conical shape was to make sure that there will be velocity gradient along the reactor body when a gas flow is applied from the bottom. The velocity gradient was created due to a change in the reactor diameter of conical shape. As seen in Figure 3.3.5, the highest velocity was obtained at the narrowest cross-section area which is bottom end. As the air moves along the reactor body toward the top, the velocity decreases. 


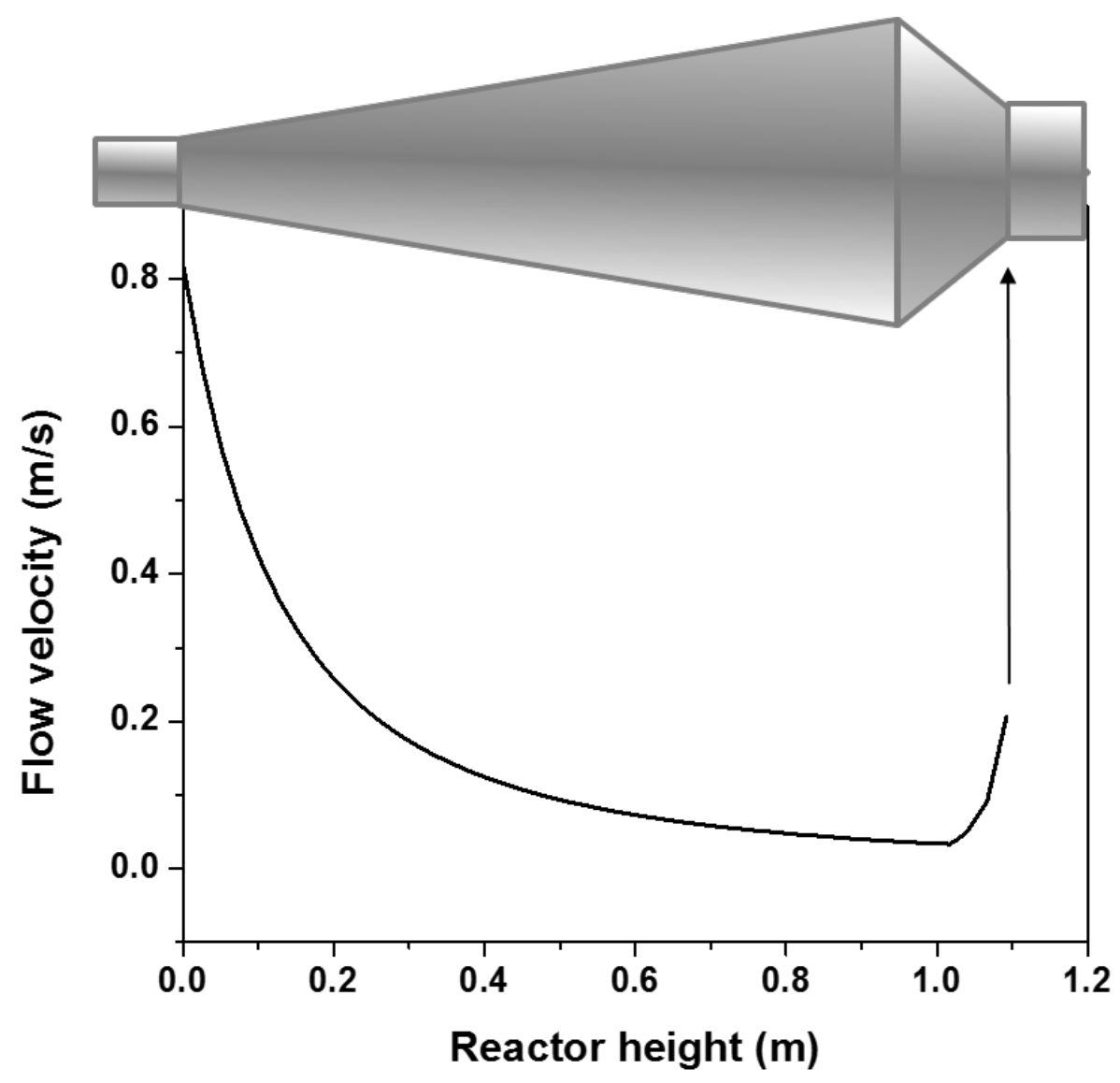

Figure 3.3.5: Velocity gradient along the reactor created by conical shape of reactor

The narrow end of $50 \mathrm{~mm}$ in diameter was to match with the current available plasma applicator. The length of reactor was $1 \mathrm{~m}$ which is about the same length as the quartz tube of the downstream plasma reactor described in section 3.2. The top unit was $250 \mathrm{~mm}$ in diameter which is the standard flange dimension. The simple model for contours of velocity magnitude (Figure 3.3.6) shows that the conical shape reactor was an ideal shape for excellent mixing. As seen in Figure 3.3.6 a, there was minimized dead area of poor mixing in conical shape reactor. The cylindrical shape reactor (Figure 3.3.6 b), however, introduced huge dead area of poor mixing. The very-wide-top conical shape 
reactor also resulted in very large dead area of poor mixing on the top portion.

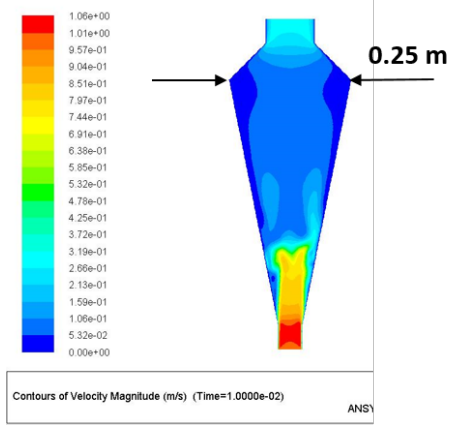

a)

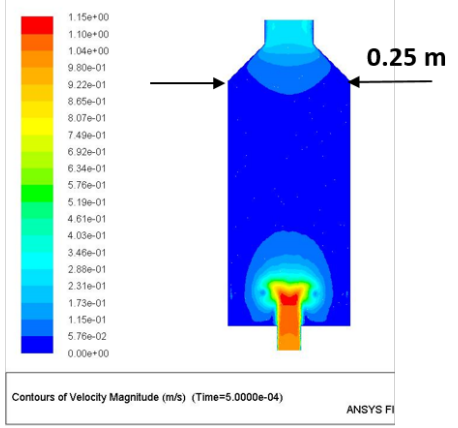

b)

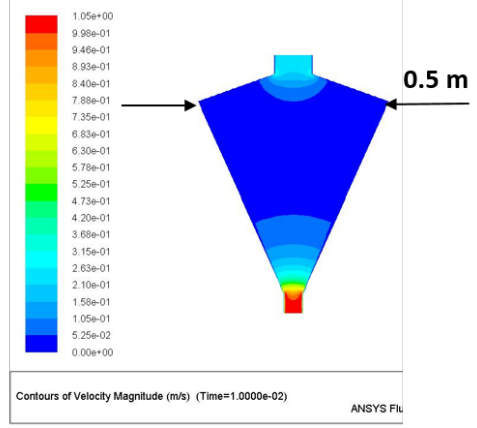

c)

Figure 3.3.6: Contours of velocity magnitude of different reactor shapes: a) conical shape, b) cylindrical shape, and c) very-wide-top conical shape

\subsection{Experimental methods}

\subsubsection{Synthesis of zinc oxide using fluidized bed reactor}

Experiments were conducted in the fluidized bed reactor using either atmospheric microwave plasma flame or hydrocarbon flames. The zinc metal powder of 1-10 microns in diameter was transferred into the powder feeder. The metal powder was entrained into the reactor from the bottom using argon as carrier gas. The plasma systems generated plasma flame into the fluidized bed reactor from the bottom either using a plasma power of $1.5 \mathrm{~kW}$, a compressed air of 20 LPM (Liter per minute) or using a custom-designed hydrocarbon burner generated hydrocarbon flame into the fluidized bed reactor. The metal powder was oxidized and converted into metal oxide nanowires under flame inside the fluidized bed reactor. A cooling air of 50-150 LPM was used to cool down gas plume coming out of reactor and transport the metal oxide nanowires to the cyclone. In the cyclone, the large metal oxide and unreacted metal particles were collected into the first 
container under the cyclone. Any fine powder coming out of the cyclone was trapped inside the filter in the filter housing unit and dropped down into a second container underneath air pressure-shock. The outlet of filter was connected to a carbon filter and any remaining particles were captured before being sent out through the exhaust. The exhaust was equipped with a carbon filter to remove any ozone coming out of reactor.

Several experiments were performed to study the effect of feeding mode, feeding rate, gas flow rate, gas composition, flame type, hydrocarbon flow rate, and burner configuration on the conversion of metal powder to metal oxide nanowires, morphology of nanowires. The experimental conditions used were summarized in Table 3.4.1. The air or oxygen used was calculated so that the flame is oxygen rich to avoid carbon soot formation. The resulting powders were characterized using a FEI Nova600 FE-SEM, a Renishaw Raman Spectroscope and a Bruker D8 Advanced Model X-ray Diffractometer. The statistic study on nanowire aspect ratio, diameter, and length distribution were performed by measuring about 100-180 nanowires on a SEM image using imageJ software.

Table 3.4.1: Summary of the experimental conditions

\begin{tabular}{|c|c|c|c|c|c|}
\hline Flame & $\begin{array}{c}\text { Feed rate, } \\
\text { g/min }\end{array}$ & $\begin{array}{c}\text { Fuel flow } \\
\text { rate, LPM }\end{array}$ & $\begin{array}{c}\text { Oxygen flow } \\
\text { rate, LPM }\end{array}$ & $\begin{array}{c}\text { Air flow } \\
\text { rate, LPM }\end{array}$ & $\begin{array}{c}\text { Plasma } \\
\text { power, kW }\end{array}$ \\
\hline Plasma & 3 & N/A & & 40 & 1.5 \\
\hline Hydrocarbon & $3, \mathbf{1 2}, 24$ & $2, \mathbf{5}, 8$ & $15,30, \mathbf{4 5}$ & $0, \mathbf{4 5}$ & N/A \\
\hline
\end{tabular}

\subsubsection{Catalyst preparation and testing for zinc oxide nanowires}




\subsubsection{Catalyst Preparation}

A slurry was made by adding DI water to $\mathrm{ZnO} \mathrm{NW}$ and $\gamma-\mathrm{Al}_{2} \mathrm{O}_{3}$. Aqueous solutions of nickel acetate $\left(\mathrm{NiCOOH} \cdot 2 \mathrm{H}_{2} \mathrm{O}\right)$ was made by adding DI water and by heating and stirring the solution at 90C. The nickel acetate solution was added drop-bydrop to the slurry while the mixture being stirred. The $\mathrm{pH}$ was maintained around 9.0 by adding aqueous solution of $\mathrm{NH}_{4} \mathrm{OH}$. Then, the mixture was dried at $80^{\circ} \mathrm{C}$ for 15 hours in a furnace and stirred once during drying. Thereafter, the mixture was dried at $150^{\circ} \mathrm{C}$ for a few hours and stirred once to obtain a thick paste for extrusion. Thereafter, the paste was extruded and dried at $150^{\circ} \mathrm{C}$ for $1 \mathrm{~h}$. Finally, they were calcined at $390^{\circ} \mathrm{C}$ for $2-2.5 \mathrm{~h}$. A batch of extrudates used in this work is shown in Figure 3.4.1. The length and diameter of these extrudates were 4-5 $\mathrm{mm}$ and 1-1.5 mm, respectively. These extrudates were then used for the hydro-desulfurization and aromatic hydrogenation tests. With the addition of molybdenum acetate, the $\mathrm{Ni}$ and $\mathrm{Mo}$ supported on $\mathrm{ZnO} \mathrm{NW}$ catalyst was also prepared using the same synthesis method.
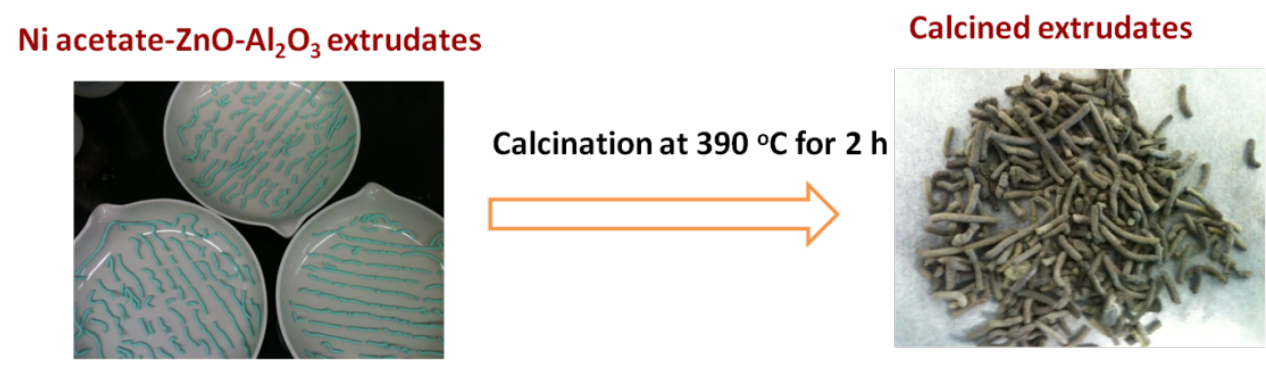

Figure 3.4.1: Extrudates of nickel acetate- $\mathrm{ZnO}-\gamma-\mathrm{Al}_{2} \mathrm{O}_{3}$ and $\mathrm{NiO}-\mathrm{ZnO}-\gamma-\mathrm{Al}_{2} \mathrm{O}_{3}$

\subsubsection{Ultra-deep desulfurization}

First, the catalyst was reduced at $430^{\circ} \mathrm{C}$ for $3 \mathrm{~h}$ with a $\mathrm{H}_{2}$ flow rate of $0.15 \mathrm{~L} / \mathrm{min}$ at atmospheric pressure. The hydro-desulfurization reaction was carried out at 30 bar and $290^{\circ} \mathrm{C}$ (furnace temperature) with $0.15 \mathrm{~L} / \mathrm{min} \mathrm{H}_{2}$ flow. The packed bed tube (1 inch 
diameter pipe) is surrounded using a vertical furnace whose temperature is continuously monitored and controlled. The diesel feed rate was $0.36 \mathrm{~mL} / \mathrm{min}$. The corresponding liquid hourly space velocity (LHSV) was $2.2 \mathrm{~h}^{-1}$ better than the standard industrial practice for ULSD [83]. A fixed bed reactor (Figure 3.4.2a) was used to carry out the reduction, aromatic hydrogenation, and hydro-desulfurization tests.

Catalysts were packed in to the reactor column shown schematically in Figure 3.4.2 (b). The extrudates were mixed with $\mathrm{SiC}$ to avoid channeling of the fuel and to keep uniform temperature throughout the bed. SiC to Catalyst weight ratio in each layer (Figure 3.4.2 (b)) was 3.5. The sulfur species composition and content was varied in our fuel feeds by spiking sulfur content in to ULSD fuels (from a refinery in Kentucky, USA) that originally contained about $9 \mathrm{ppm}$ of sulfur. The treated samples were collected every few hours during the test. In order to gain insight into the origin of catalytic activity and the composition after desulfurization, both pre-run (fresh) and post-run (spent) catalysts were characterized using BET, scanning electron microscopy (SEM), transmission electron microscopy (TEM), and X-ray diffraction (XRD). Total aromatics were determined using a UV-Vis spectrometer. The morphology of the catalysts was studied using a field-emission FEI Nova 600 NanoLab scanning electron microscope (SEM), Bruker Discover D8 powder X-ray diffraction (XRD) system. The identification of the crystal phases was performed using the Powder Diffraction File (PDF) structural database.

An Antek 9000 VS Sulfur analyzer was used to measure sulfur concentration in all the samples. The operation begins with complete high temperature oxidation of the entire sample matrix. The sample is vaporized and combined with oxygen at a 
temperature of $1250^{\circ} \mathrm{C}$ to convert chemically bound sulfur to $\mathrm{SO}_{2}$. The $\mathrm{SO}_{2}$ is exposed to ultraviolet radiation of a specific wavelength and the resulting fluorescent emission is completely specific for sulfur and is proportional to the amount of sulfur in the original sample.

(a)

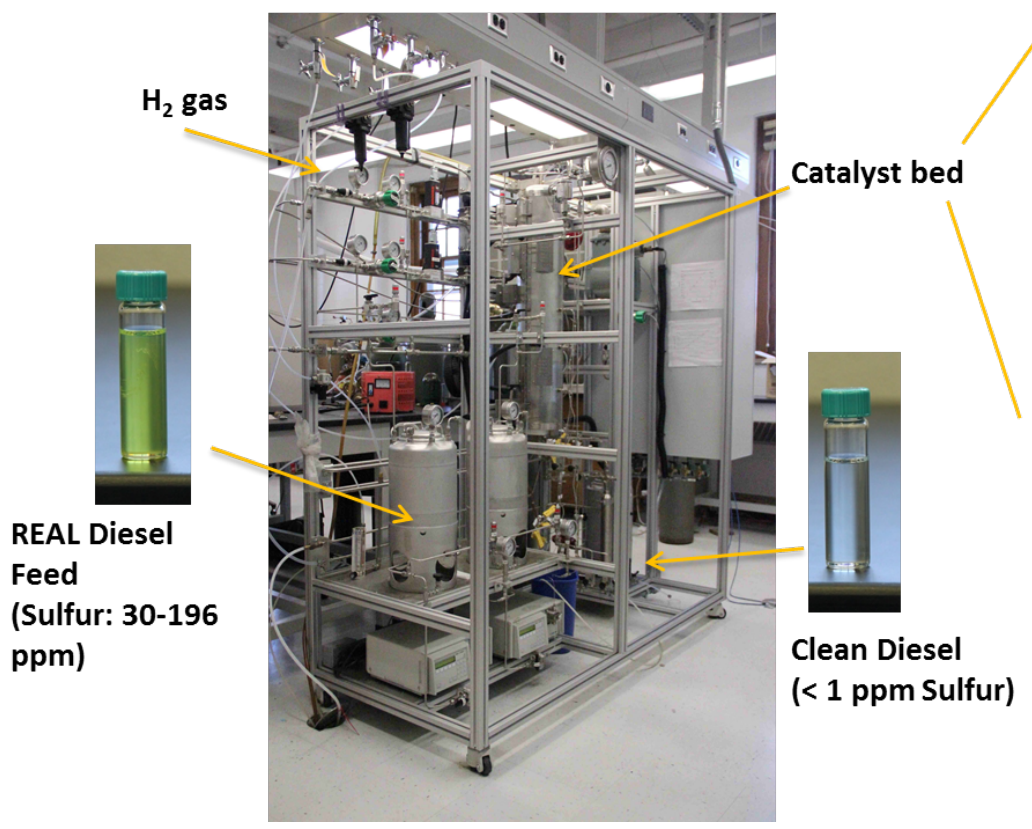

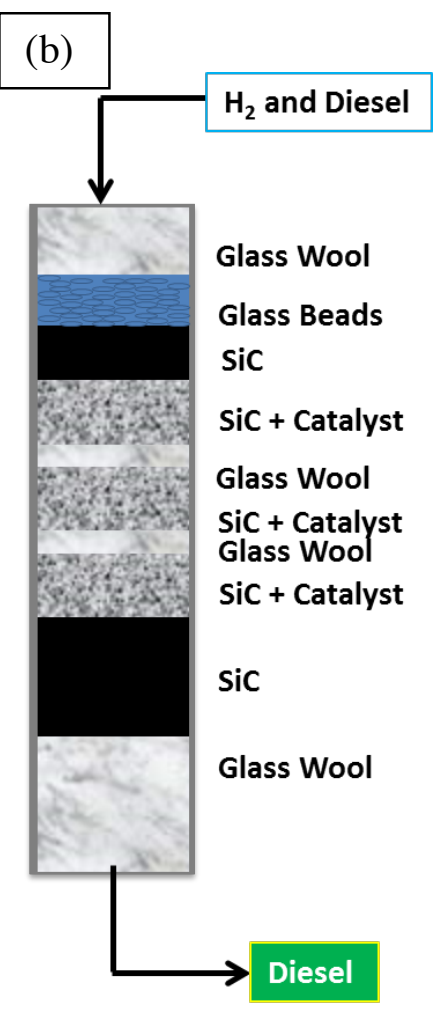

Figure 3.4.2: (a) Photograph of the fixed bed reactor used for desulfurization experiments; (b) Schematic of three zone packing design used in our packed column.

\subsubsection{Synthesis of metal oxide using upstream plasma reactor via solvo plasma}

Metal oxide nanowires are synthesized in an upstream microwave plasma reactor whose details have been described in detail elsewhere [16]. Briefly, metal/metal oxide 
particles $(1 \div 5 \mu \mathrm{m}$ size, Atlantic Equipment Engineer, Inc) are mixed with $\mathrm{KOH} / \mathrm{KCl}$ powder in a 3:1 ratio by weight and water is added to make the precursor paste. The precursor paste is then sprinkled on the top of a $25 \times 25 \mathrm{~mm}$ quartz substrate, which is then carefully exposed to the plasma flame at power of 1.0-1.4 kW, $81 \mathrm{pm}$ of Air flow for 10 seconds to $5 \mathrm{~min}$. The as-synthesized materials are dispersed in $0.1 \mathrm{M} \mathrm{HCl}$ solution for $1 \mathrm{~h}$ to facilitate ion exchange, washed with deionized water, and annealed by exposure to plasma flame for 1 to $5 \mathrm{~min}$.

\subsubsection{Detail of computational fluid mechanics simulations}

\subsubsection{Meshing}

The actual simplified 3D reactor geometry was used. Figure 3.4.3 shows the meshing setup and the reactor geometry after the mesh generation. The top and bottom surface were defined as outlet and inlet. All the other surfaces were defined as wall. Select "Fine" for relevance center, and "High" for smoothing. The meshing was controlled to have minimum sizes of $0.002 \mathrm{~m}$, maximum face sizes of $0.0025 \mathrm{~m}$ and maximum sizes of $0.0030 \mathrm{~m}$. The mesh consists of 426,998 mixed cells. No mesh sensitivity analysis was performed; therefore, results are just an approximation. 


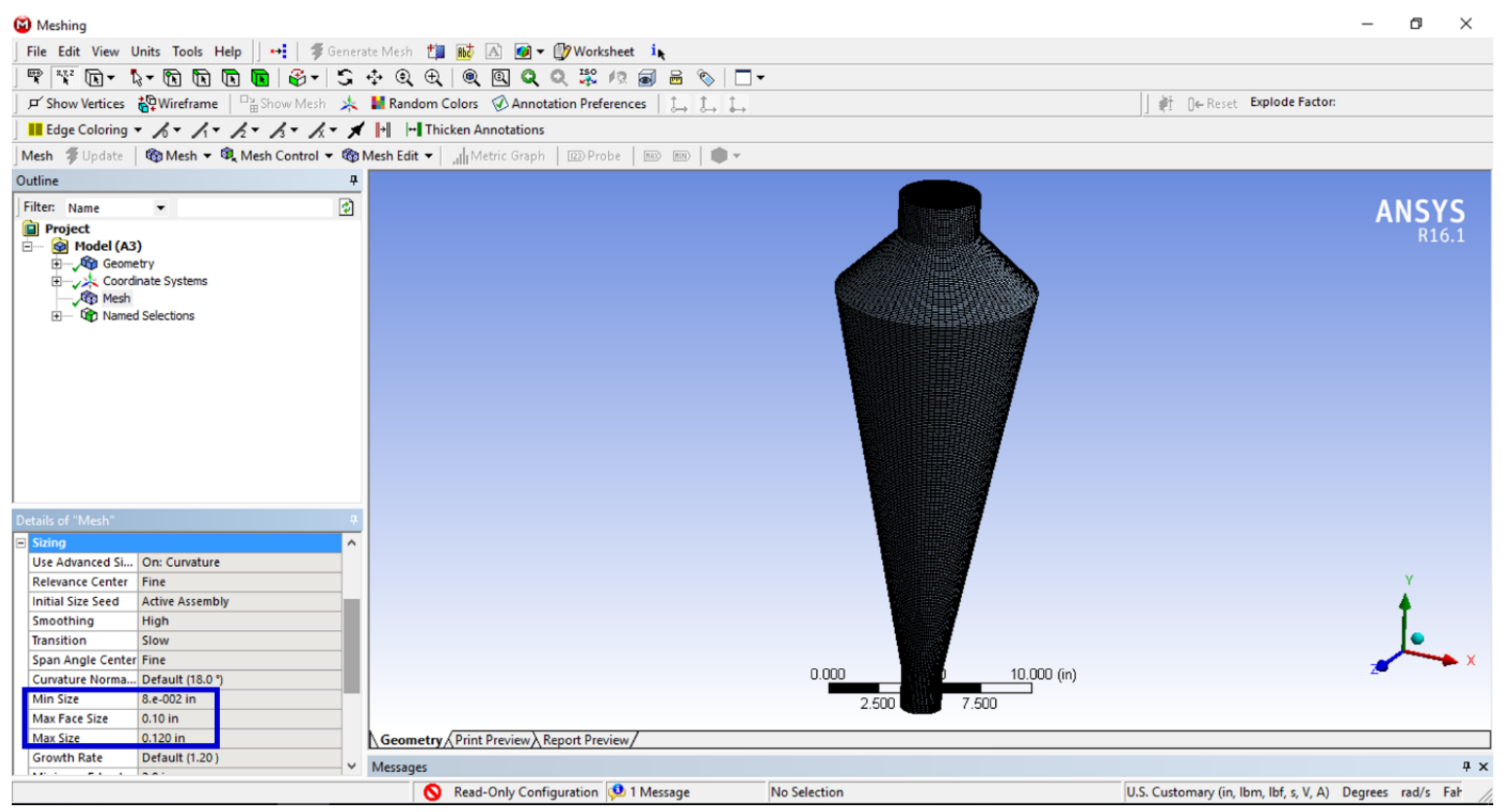

Figure 3.4.3: Meshing size and the reactor geometry after the mesh generation

\subsubsection{Simulation setup}

In the general menu, select "Transient" and put "-9.81" in Y-component of gravitational acceleration. Select "Pressure based" and "Absolute" for Type and Velocity formulation, respectively.

In model menu, select "Eulerian" as Multiphase Model. Number of Eulerian Phases was set at 1. Number of Discrete Phases was set at 1. Select "Reynolds Stress" as Viscous Model. The model constants are 0.09 for $\mathrm{cmu}, 1.44$ for C1-epsilon, 1.92 for C2epsilon, 1.8 for C1-PS, 0.6 for C2-PS, 0.5 for C1'-PS, 0.3 for C2'-PS, 1 for TKE Prandtl number, 1.3 for TDR Prandtl number, and 0.75 for Dispesion Prandtl number. Select “Update DPM sources every flow iteration", "Unsteady Particle Tracking” and "Track with fluid flow time step" in Discrete Phase model. Input 50,000 for Max number of steps. Click "Injections" tab to setup injection. Select "surface" for Injection type, "inlet" for Release from surface. Input " 1 " for velocity magnitude, " $5 \times 10^{-8}$ " for min diameter, 
" $5 \times 10^{-5}$ " for max diameter, " $5 \times 10^{-6 "}$, for mean diameter, and " 3 " for number of diameters.

In materials menu, select "Air" for fluid. 1.225 and $1.7894 \mathrm{e}-05$ were density and viscosity of air, respectively. Input "7140" for inert particle density which represents the density of zinc metal.

In boundary conditions menu, select "velocity-inlet" for type for inlet's boundary condition. Turbulent intensity and turbulent viscosity were 5\% and $10 \%$, respectively. Select "outflow" for outlet's boundary condition. Flow rate weighting for outflow was 1.

Phase coupled simple was selected under Scheme for solution method.

Solution control were default values as 0.3 for pressure, 1 for density, 1 for body forces, 0.7 for momentum, 0.5 for volume fraction, 0.2 for granular temperature, 0.8 for turbulent kinetic energy, 0.8 for turbulent dissipation rate, 1 for turbulent viscousity, 0.5 for Reynolds stresses, and 0.5 for discrete phase sources.

Hybrid initialization was used for solution initialization.

In the run calculation menu, input " 0.01 ” for time step size, " 2000 " for number of time steps, and " 10 " for max iterations/Time step reporting interval.

The steady state model was used for flow pattern. The same meshing was used. The inlet's boundary condition was set at different velocity magnitudes of 1, 10 and 40 $\mathrm{m} / \mathrm{s}$. Select "Magnitude, normal to boundary" for velocity specification method. Select "SIMPLE" for solution methods. Input "500" for Run calculation.

No parameters were monitored for convergence; therefore, the numeric values of the results are not fully reliable. However, the model can be used for predicting the general trend such as residence time and flow patterns. 


\subsection{Fabrication of lithium ion battery coin cells}

\subsubsection{Electrode preparation}

The electrode materials were prepared using $70 \mathrm{wt} \%$ active materials with $20 \mathrm{wt} \%$ AB (acetylene black) and $10 \%$ PVDF binder in NMP. The well-mixed slurry was casted onto a copper foil using a doctor blade. The electrode is dried at $180^{\circ} \mathrm{C}$ for $4 \mathrm{~h}$ under vacuum and then transferred to an argon filled glove box. The typical loading of active materials is 0.2 to $0.5 \mathrm{mg} / \mathrm{cm}^{2}$.

The tin oxide nanowires were deposited directly on a stainless steel spacing substrate which acts as electrode used for protective coating in chapter 6 .

\subsubsection{Coin cell assembly}

A CR2013 coin-type cell assembled in a dry argon-filled glove box. As shown in Figure 3.5.1, a half-cell assembly includes coin cell casings, spacer, electrode, spring, precut separators, and precut lithium metal disc as cathode. A spacer is placed into the anode coin cell casing. The electrode prepared in 3.5.1 is placed on top of placer. Two precut porous propylene films (ADVANTEC GB-100R) are placed on top of the electrode as separators. A couple of drops of electrolyte, enough to soak the separator are placed on the separator. A 1M solution of LiPF6 dissolved in an ethylene carbonate (EC) and dimethyl carbonate mixture $(\mathrm{DMC})$ (Volume ratio of $\mathrm{EC}$ : $\mathrm{DMC}=1: 2$ ) is used as the electrolyte. A precut lithium metal disc is placed on top of the separator. Another spacer is placed on top of the lithium metal disc. The spring and the cathode casing are stacked on top of the spacer. The whole assembly is placed under a crimping machine and the 
coin cell is crimped to seal battery core in the case. The electrochemical measurements are performed using a battery tester (16 Channel Arbin Instruments, USA).

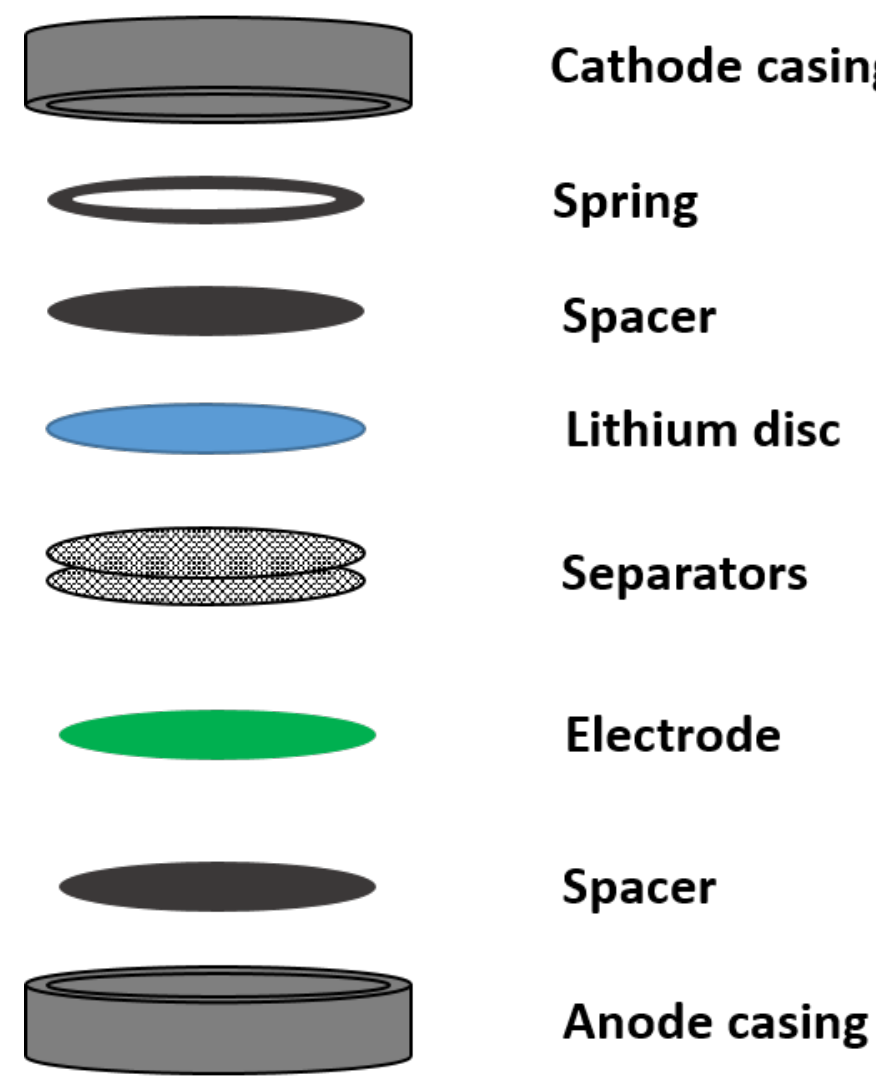

Figure 3.5.1: Schematic of lithium ion coin cell assembly

\subsection{Atomic layer deposition (ALD)}

Atomic layer deposition is a chemical vapor deposition method. However, the reaction is divided into two half-reactions. Each half-reaction is self-limiting and surface only. During each half-reaction, a precursor is pulsed to reactor chamber for a fixed amount of time to allow the precursor to fully react at the substrate and form a monolayer. The reactor chamber is purged before the next pulse of the reactant gas is introduced in order to remove unreacted species and reaction by-products. 
A commercially available ALD reactor from Cambridge instruments (Savannah 200) was used for the deposition of thin alumina and titania films on tin oxide nanowires as in Figure 3.6.1. The reactor assembly comprises of a stainless steel chamber with a heater to heat the substrate. Pulses of precursors are delivered to the reactor chamber by solenoid valves using nitrogen as carrier gas. Aluminum trimethyl and titanium tetraisopropoxide along with water were used as precursors for the deposition of alumina and titania, respectively. Nitrogen flow rate of $20 \mathrm{sccm}$ is typically used. Pressure in the chamber is monitored by a Pirani gauge. The by-products are exhausted into fume hood using a rotary vane pump. A film thickness of $0.1 \mathrm{~nm}$ can be obtained in one cycle. Thus, the number of cycle can be used for anticipating the film thickness.

\subsection{Materials characterization: Electron Microscopy and Diffraction Techniques}

The morphology of the precursors and synthesized nanowires has been characterized using scanning electron microscope (SEM) (FEI Nova 600) and transmission electron microscope (TEM) (Tecnai F20 FEI TEM operating at $200 \mathrm{kV}$ ). The composition of synthesized samples was studied using Energy Dispersive Spectroscopy (EDS) and X-rays Diffraction (XRD) (Bruker D8 Discovery with $\mathrm{Cu} \mathrm{K} \alpha$ radiation). TEM and XRD have also been performed on the samples to examine the phase and crystallinity.

\subsection{Summary}

The limitation on the scalability of nanowire production using the downstream atmospheric microwave reactor has been investigated. The reactor design modification has been performed to increase the yield and nanowire quality. An upward conical fluidized bed employing atmospheric microwave and hydrocarbon flames is designed and 
operated for large scale production of metal oxide nanowires. The fluidization in a fluidized bed reactor has been shown to increase the residence time and thus it helps to increase the reaction conversion. The resulting materials are characterized using SEM, EDS, TEM, and XRD. 


\section{CHAPTER IV}

\section{MASS PRODUCTION STUDIES OF ZINC OXIDE NANOWIRES USING A FLUIDIZED BED REACTOR}

\subsection{Introduction}

Nanowires have shown an improvement in performance at order of magnitude in a variety of applications such as catalyst supports [5, 6], absorbents [7], batteries [6], solar cells [8], capacitors, sensors [9], and polymer composites [10]. However, all of them are tested at lab scale using very small quantities. None of them have been demonstrated in large scale like prototyping or industrial scale yet due to lack of methods for producing bulk quantities of nanowire based materials. Thus, large scale production with controlled properties such as crystallinity, composition and morphology is crucial for the on-going development of nanowire based methods.

In this chapter, a new fluidized bed reactor capable of producing up to 10 kilograms per day of metal oxide nanowires is described. The investigation includes the effect of operating parameters on nanowire properties such as composition, crystallinity, morphology and overall yield of the process. 


\subsection{Fluidization and modeling}

\subsubsection{Fluidization in a fluidized bed reactor}

In gas-solid system, the particle sizes have strong effects on the dynamics behavior of gas-solid flow. Particles used in gas-solid flows are typical non-spherical or irregular shape. Sauter's particle diameter or surface volume diameter, is defined as the diameter of a sphere having the same ratio of external surface to volume as the particles, is one of the best estimation in field of reaction engineering especially in fluidizing bed systems [84].

$$
\frac{V}{A}=\frac{\frac{4}{3} \pi\left(\frac{d_{p}}{2}\right)^{3}}{4 \pi\left(\frac{d_{p}}{2}\right)^{2}}=\frac{d_{p}}{6} \rightarrow d_{p}=\frac{6 V}{A}
$$

where $\mathrm{V}$ : volume of particle, $\mathrm{m}^{3}$

A: surface area of particle, $\mathrm{m}^{2}$

$\mathrm{d}_{\mathrm{p}}$ : diameter of particle, $\mathrm{m}$

In the fluidized bed system, there is a drag force exerted on the particles by the flowing gas. When the gas velocity is increased to a certain value, the drag on the particles will be equal the gravitational force exerted on particles, and the particles will begin to lift and fluidize. The velocity of gas at this point is called minimum fluidization velocity of particles $\left(\mathrm{u}_{\mathrm{mf}}\right)$. As the gas velocity increased further, the particles started being carried out of the reactor. At this point, the velocity of gas flow is terminal settling velocity $\left(\mathrm{u}_{\mathrm{t}}\right)$ [85]. The terminal settling velocity and minimum fluidization velocity are calculated in the following equations 


$$
\begin{aligned}
& u_{t}=\sqrt{\frac{4 g d_{p}\left(\rho_{p}-\rho\right)}{3 \rho C_{D}}}(6-229 \text { Perry's Handbook) (4.2.2) } \\
& u_{m f}=\frac{\left(d_{p}\right)}{150 \mu}\left[g\left(\rho_{p}-\rho\right)\right] \frac{\varepsilon_{m f}^{3}}{\left(1-\varepsilon_{m f}\right)^{2}}
\end{aligned}
$$

where $\mathrm{u}_{\mathrm{t}}$ : terminal settling velocity, $\mathrm{m} / \mathrm{s}$

$$
\begin{aligned}
& \mathrm{u}_{\mathrm{mf}}: \text { minimum fluidization velocity, } \mathrm{m} / \mathrm{s} \\
& \mathrm{d}_{\mathrm{p}} \text { : particle diameter, } \mathrm{m} \\
& \rho_{\mathrm{p}} \text { : particle density, } \mathrm{kg} / \mathrm{m}^{3} \\
& \rho: \text { fluid density, } \mathrm{kg} / \mathrm{m}^{3} \\
& \mathrm{C}_{\mathrm{D}} \text { : drag coefficient } \\
& \mu: \text { viscosity of fluid, } \mathrm{cp} \\
& \varepsilon_{\mathrm{mf}}: \text { void fraction }
\end{aligned}
$$

Table 4.2.1 shows the effect of particle size of both feeding and final product on the terminal settling velocity and minimum fluidization velocity. The larger particle size requires higher terminal velocity to keep them fluidizing in the reactor for feeding materials or to carry them out of the reactor for final product materials. As a result, large particles will experience a longer residence time than those of smaller particles.

Table 4.2.1: Effect of particle size on terminal settling velocity 


\begin{tabular}{|c|c|c|c|c|c|}
\hline & \multicolumn{2}{|c|}{ Zn (feed) } & \multicolumn{3}{c|}{ ZnO (product) } \\
\hline Particle size & $5 \mu \mathrm{m}$ & $1 \mu \mathrm{m}$ & $1 \mu \mathrm{m}$ & $0.5 \mu \mathrm{m}$ & $200 \mathrm{~nm}$ \\
\hline $\mathrm{Re}=\frac{\rho \mu_{\infty} D_{p}}{\mu}$ & 148.32 & 29.66 & 25.33 & 12.66 & 5.06 \\
\hline $\mathrm{C}_{\mathrm{D}}$ & 0.91 & 2.02 & 2.21 & 3.46 & 6.80 \\
\hline $\mathrm{u}_{\mathrm{t}}(\mathrm{m} / \mathrm{s})$ & 1.30 & 0.39 & 0.34 & 0.19 & 0.09 \\
\hline $\mathrm{u}_{\mathrm{mf}}(\mathrm{m} / \mathrm{s}) \times 10^{3}$ & 16.67 & 0.67 & 0.57 & 0.14 & 0.02 \\
\hline
\end{tabular}

\subsubsection{Modeling}

Modeling the fluid flow in the fluidized bed reactor was performed to understand the effect of fluid flows on flow pattern, and to understand the fluidization of particles. Commercially available ANSYS FLUENT 16.1 software was used for the modeling.

Figure 4.2.1 shows contours of velocity magnitude at different inlet velocity. At high inlet velocity of $40 \mathrm{~m} / \mathrm{s}$, there was a velocity gradient along the reactor height due to inherent conical shape of reactor. The maximum velocity was obtained at the 2" inlet. The minimum velocity was achieved at the widest portion of the reactor. The velocity gradient decreased with decreasing inlet velocity. As a variety of particle size (1-10 microns) is being used in our process, a velocity gradient along the reactor must be presented to meet the requirements of terminal settling velocity for the fluidization. Therefore, a conical shape of reactor seems to be an ideal case for the fluidization. 


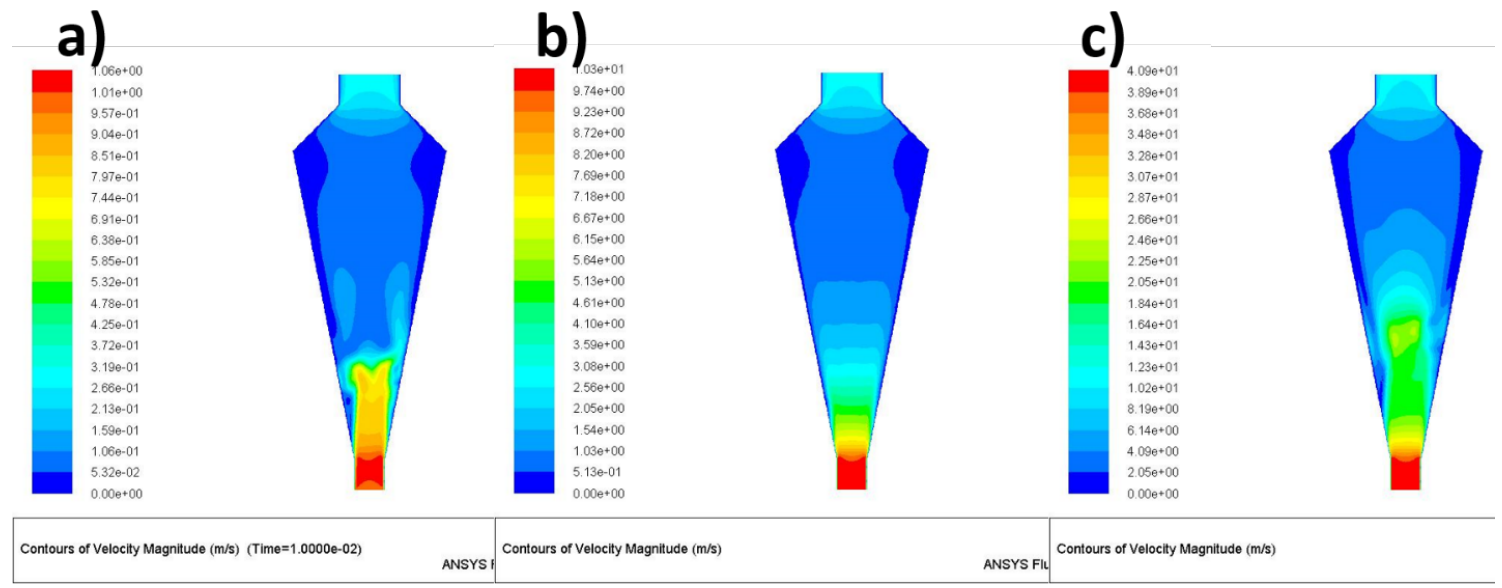

Figure 4.2.1: Contours of velocity magnitude at different flow inlet velocities of a) $1 \mathrm{~ms}^{-}$ , a) $10 \mathrm{~ms}^{-1}$, and a) $40 \mathrm{~ms}^{-1}$

As a closed look at the streamlines in Figure 4.2.2, the flow velocity was decreasing from inlet to the top outlet. At any cross section, the highest flow velocity was obtained at the center line of the reactor. The velocity was low as approaching the reactor wall due to friction. There are also stagnation areas at the widest portion of the reactor where the flow is highly turbulent. 


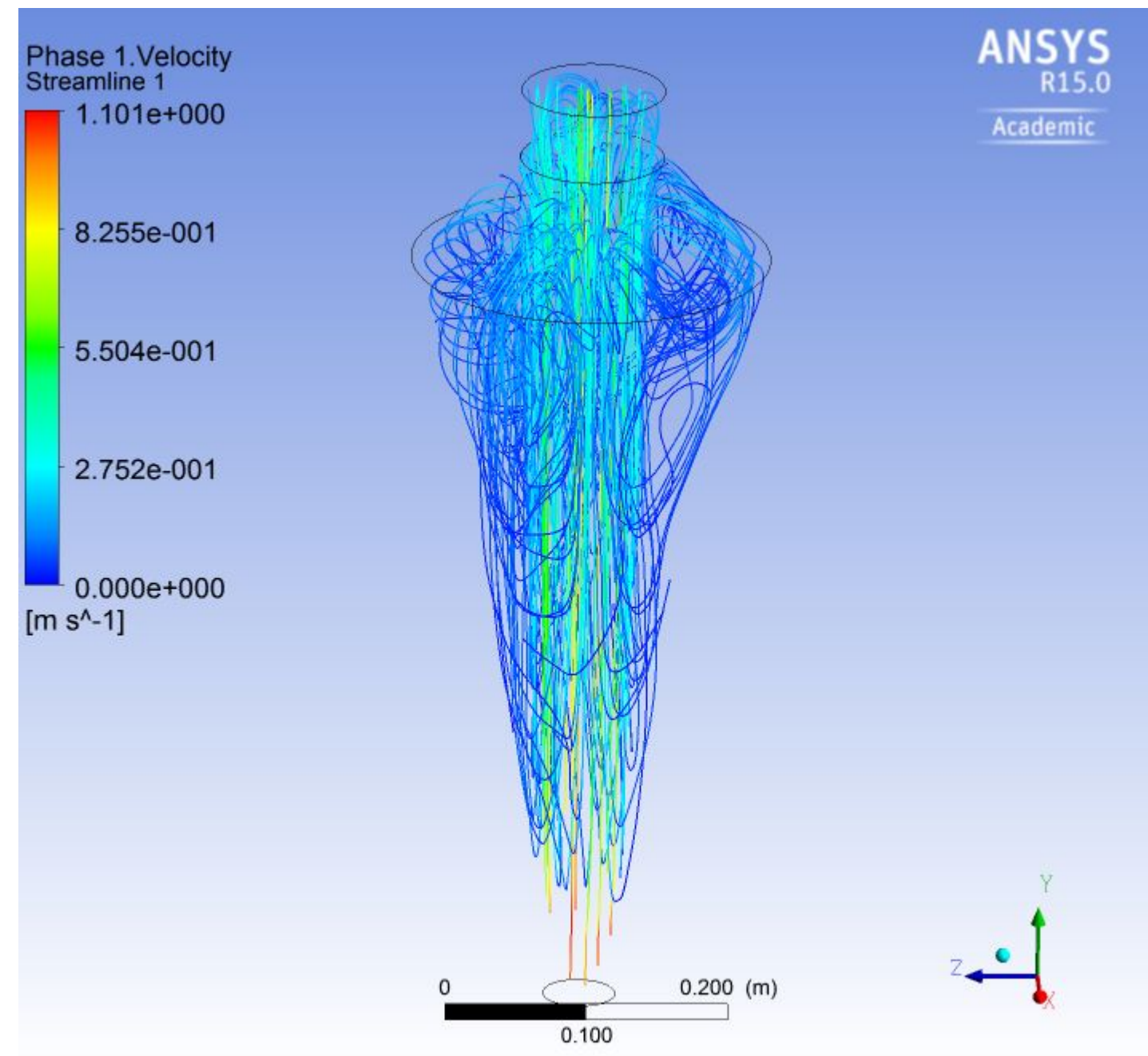

Figure 4.2.2: Streamlines: Colored by velocity magnitudes

A simple particle tracking model in transient mode was also developed to study the fluidization in our fluidized bed reactor. As shown in Figure 4.2.3, 2986 particles of two different sizes of 12.5 microns (red particles) and $50 \mathrm{~nm}$ (blue particles) have been injected at the inlet of the fluidized bed reactor. The difference in moving speed could be seen clearly at 0.5 seconds. The larger red particles tended to move slower than the smaller blue particles due to gradient velocity as discussed in previous paragraph. As time proceeded, the small blue particles also tended to occupy mostly at the top portion of the reactor while the large red particles remained mostly at the bottom portion. As in 
second cycle, the red particles fell down closer to the inlet while the blue particles fell down only to a certain height above the inlet before got blown up by a high velocity flow closer to the inlet (see at 10 seconds). The particles kept being blown up and fell down unless they exited at the outlet. Eventually, 1704 particles left after 20 seconds. The model shows a residence time of 20 seconds. The "20 seconds" was not an exact number. In order to obtain an exact number, parameters must be monitored for convergence However, it shows that the fluidization of particles could increase the residence time due to an increase in distance that the particles traveled inside the reactor.

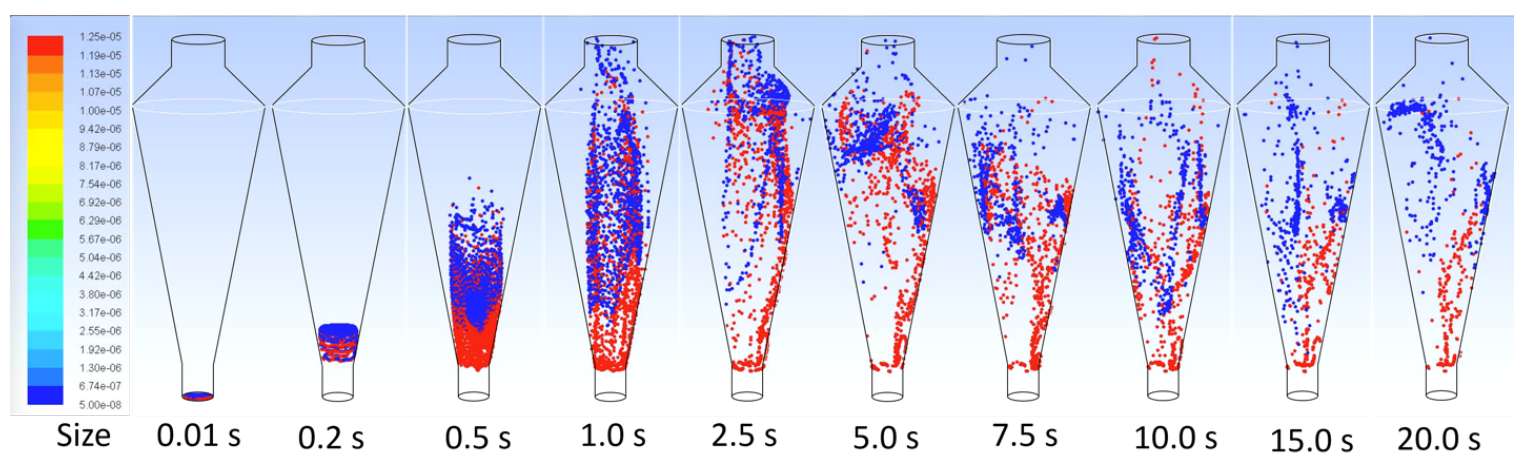

Figure 4.2.3: Particle tracking: Colored by particle size

In reality, our starting materials are zinc particles of about 1-5 microns. During the reaction, the heavy zinc metal particles are converted to zinc oxide nanowires that have lighter density than zinc metal particles. The zinc oxide nanowire products have the equivalent particle diameters of $200-1000 \mathrm{~nm}$. As shown in Table 4.2 .1 calculations of minimum fluidization and terminal settling velocity for different particle sizes, a minimum inlet velocity of $16.67 \times 10^{-3} \mathrm{~m} / \mathrm{s}$ is required for the fluidization of both starting materials and products. The minimum fluidization velocity depends mainly on particle density and particle size (equation 4.2.3). The required minimum fluidization velocity 
increases with increasing particle density and particle size.

Once the flow velocity was equal to or higher than minimum fluidization velocity, the fluidization occurred. Then the flow velocity can be used to control the fluidization. As seen in the equation 4.2.2, the terminal settling velocity depends mainly on particle size, particle density and drag coefficient. The terminal settling velocity also increases with increasing particle density and particle size. In order to transfer particle out of the reactor at the outlet, the velocity at the outlet must be higher than the terminal settling velocity of the particle. In order to keep particle fluidizing in the reactor for longer time, the flow velocity at the outlet must be smaller than the terminal settling velocity of the particle. Therefore, the extent of fluidization can be varied using flow rate, particle diameter, and particle density parameters. In this particular study of zinc particles and zinc oxide nanowires, the estimated flow velocity at outlet of reactor must be greater than $0.34 \mathrm{~m} / \mathrm{s}$ and smaller than $0.39 \mathrm{~m} / \mathrm{s}$ (see Table 4.2 .1 ). These flow velocity parameters are used to make sure that the product can be easily transferred out at the outlet but the zinc metal are still remaining in the reactor for the reaction.

In any fluidization system, it is important to know the maximum solid load. The maximum solid loading can be calculated using the following equation:

$$
m=\rho \times\left(V_{f} \times A\right) \times\left(1-f_{v}\right)
$$

where

$\rho$ : density of the solid, $\mathrm{kg} / \mathrm{m}^{3}$

Vf: fluidization velocity, $\mathrm{m} / \mathrm{s}$ 
A: cross-section area of reactor, $\mathrm{m}^{2}$

fv: void fraction

\subsection{Production of zinc oxide nanowires using atmospheric microwave plasma}

Figure 4.3.1 shows the SEM image of zinc oxide nanowires collected under cyclone and filter. The powder collected under the cyclone contained a lot of unreacted zinc metal powder. The nanowires are about $20-80 \mathrm{~nm}$ in diameter and $400 \mathrm{~nm}$ to 1 micron in length. The statistical measurement in Figure 4.3.2 shows that nanowires had an aspect ratio of 5 to 20 . The yield of about $30 \%$ was calculated by comparing the powder collected under the cyclone and filter housing.
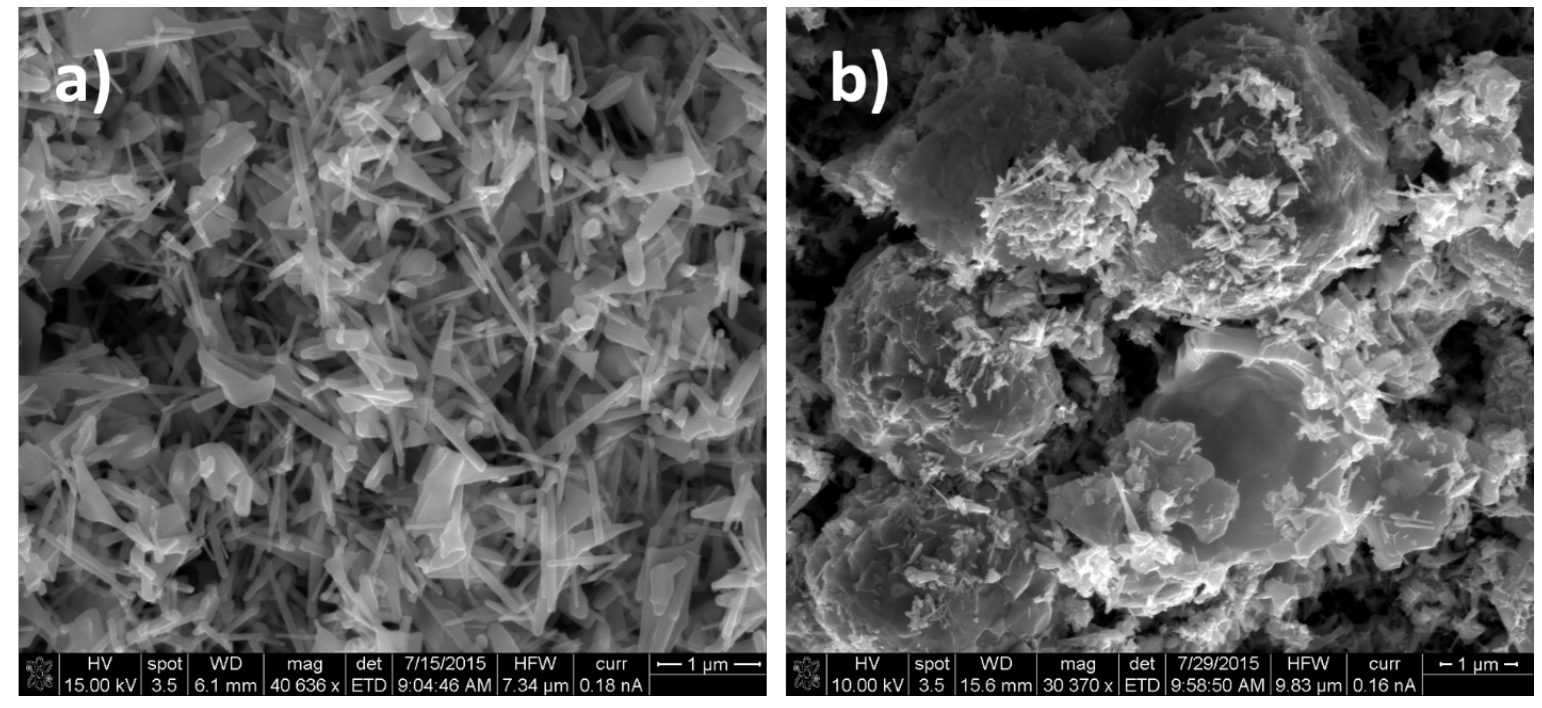

Figure 4.3.1: SEM image of zinc oxide nanowires collected under the filter a) and under cyclone b) (Plasma power: $1.5 \mathrm{~kW}$. Air: $20 \mathrm{LPM}+1 \mathrm{CFM}+5 \mathrm{CFM})$ 

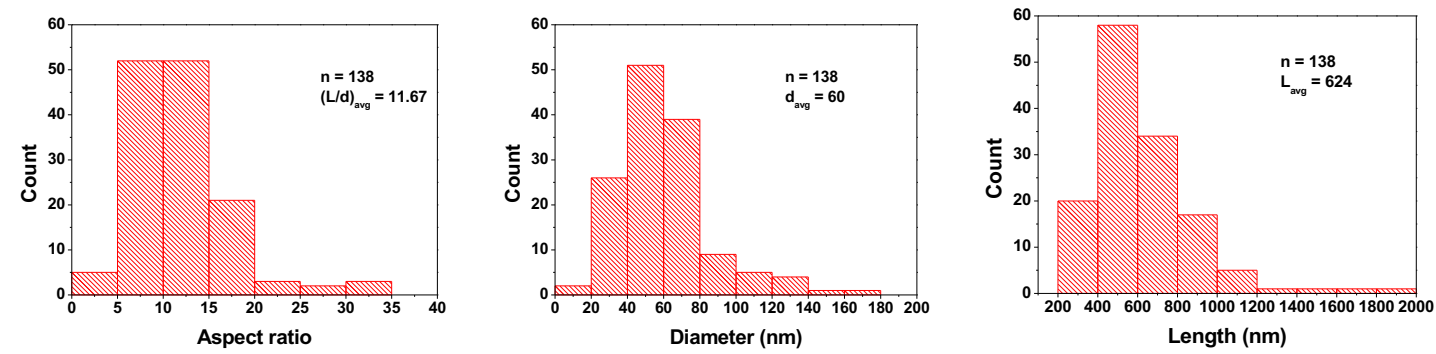

Figure 4.3.2: Aspect ratio, diameter and length distributions of zinc oxide produced using plasma

\subsection{Production of zinc oxide nanowires using hydrocarbon flame}

Figure 4.4.1 shows SEM images of zinc metal particles as starting materials and resulting zinc oxide nanowires. The zinc oxide appeared to be short nanowires of about 1 micron in length and $50-200 \mathrm{~nm}$ in diameter. The TEM images shows that the resulting zinc oxide are single crystalline as shown in Figure 4.4.1 c and d. Figure 4.4.2 also confirms that the resulting powder is pure phase zinc oxide nanowires. All of the peaks are assigned to wurtzite hexagonal zinc oxide[86]. The BET surface area was $7.37 \mathrm{~m}^{2} / \mathrm{g}$. The EDS analysis on the sample collected under the filter shows that there is no carbon contamination which is a common drawback in hydrocarbon based synthesis technique. In order to control the nanowire properties, the following discussion describes the effect of operating parameters on nanowire morphology and yield. 

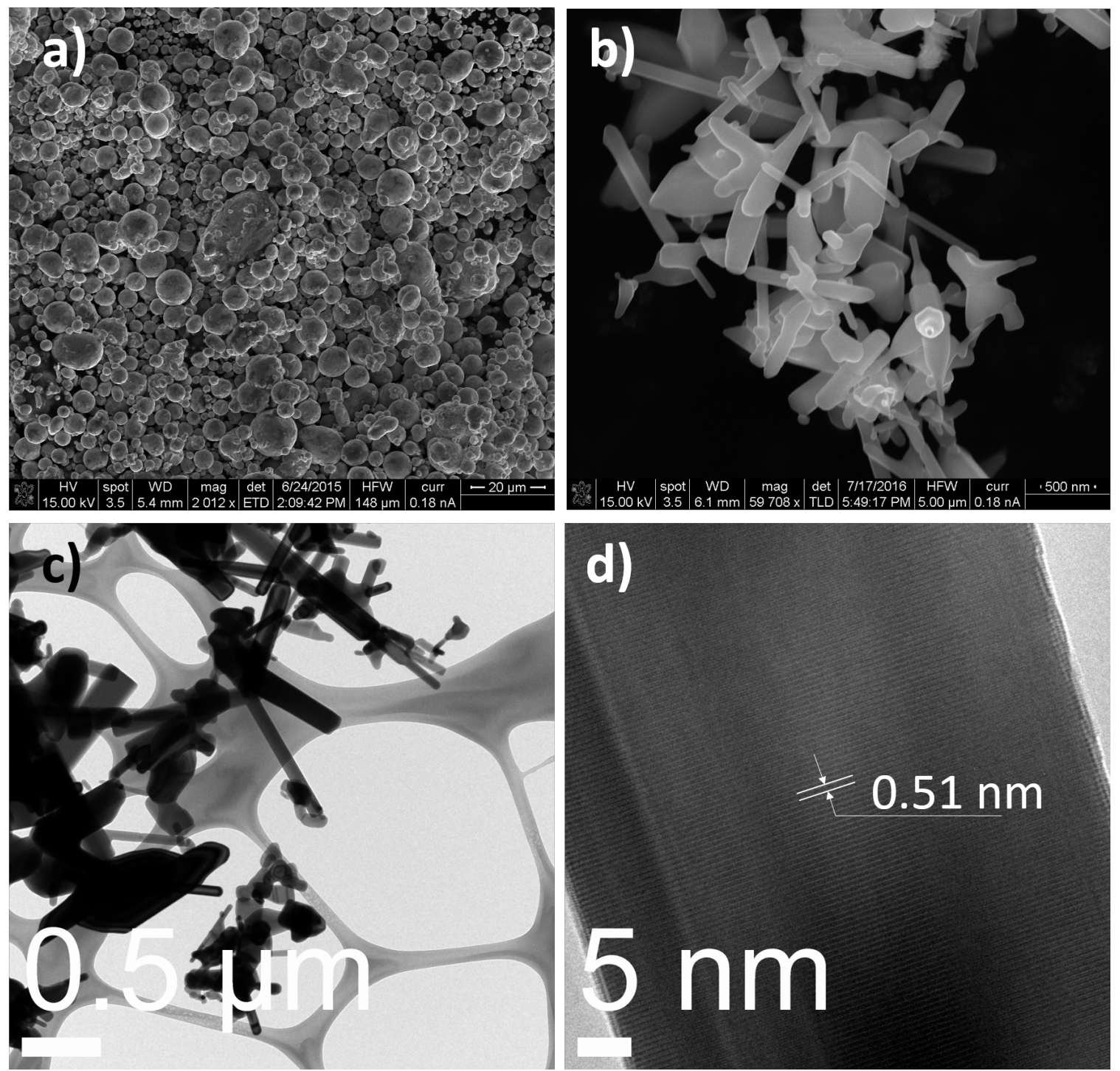

Figure 4.4.1: SEM image of zinc metal particles as starting materials a), SEM image of typical resulting zinc oxide nanowires b); TEM image of typical resulting zinc oxide nanowires c); HR-TEM image of typical resulting zinc oxide nanowires d) 


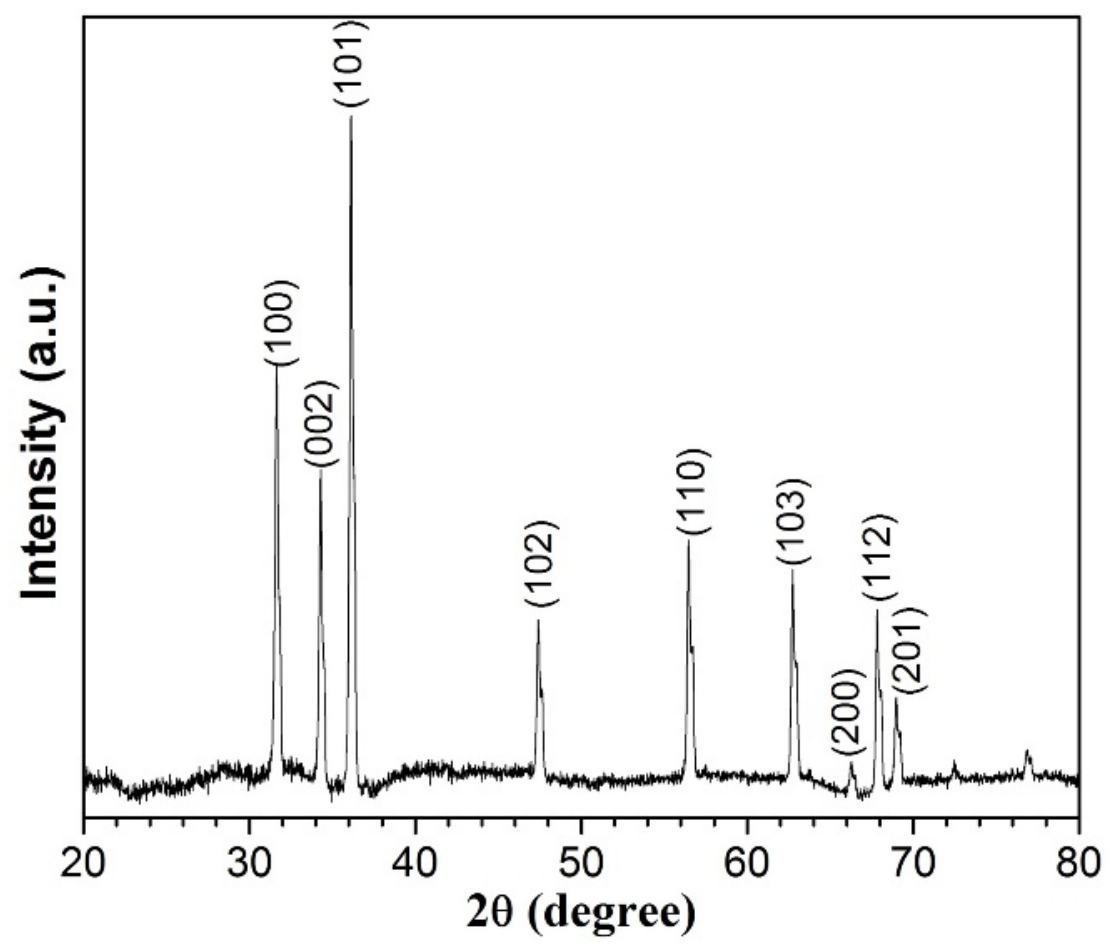

Figure 4.4.2: XRD pattern of resulting zinc oxide nanowire powder

\subsubsection{Effect gas flow rates}

In a gas-solid system, the gas flow rate has a significant effect on the fluidization. High oxygen flow rate results in short residence time and low flame temperature. However, high hydrocarbon flow rate provides longer flame and relative long residence time.

Figure 4.4.3 shows SEM images of zinc oxide nanowires produced using different oxygen flow rates. The nanowire diameter increased from 80 to $130 \mathrm{~nm}$ as the oxygen flow increased from 15 to 45 LPM. However, the aspect ratio was decreased from 9.10 to 4.53 as the oxygen flow increased from 15 to 45 LPM (see Figure 4.4.4). The same observation on decreasing aspect ratio was reported previously for zinc oxide nanorods $[63,68]$. Lot of unreacted zinc metal particles was also seen at low oxygen flow rate of 
15 LPM. 15 LPM of oxygen provides insufficient amount of oxygen for complete reaction of zinc metal to zinc oxide.
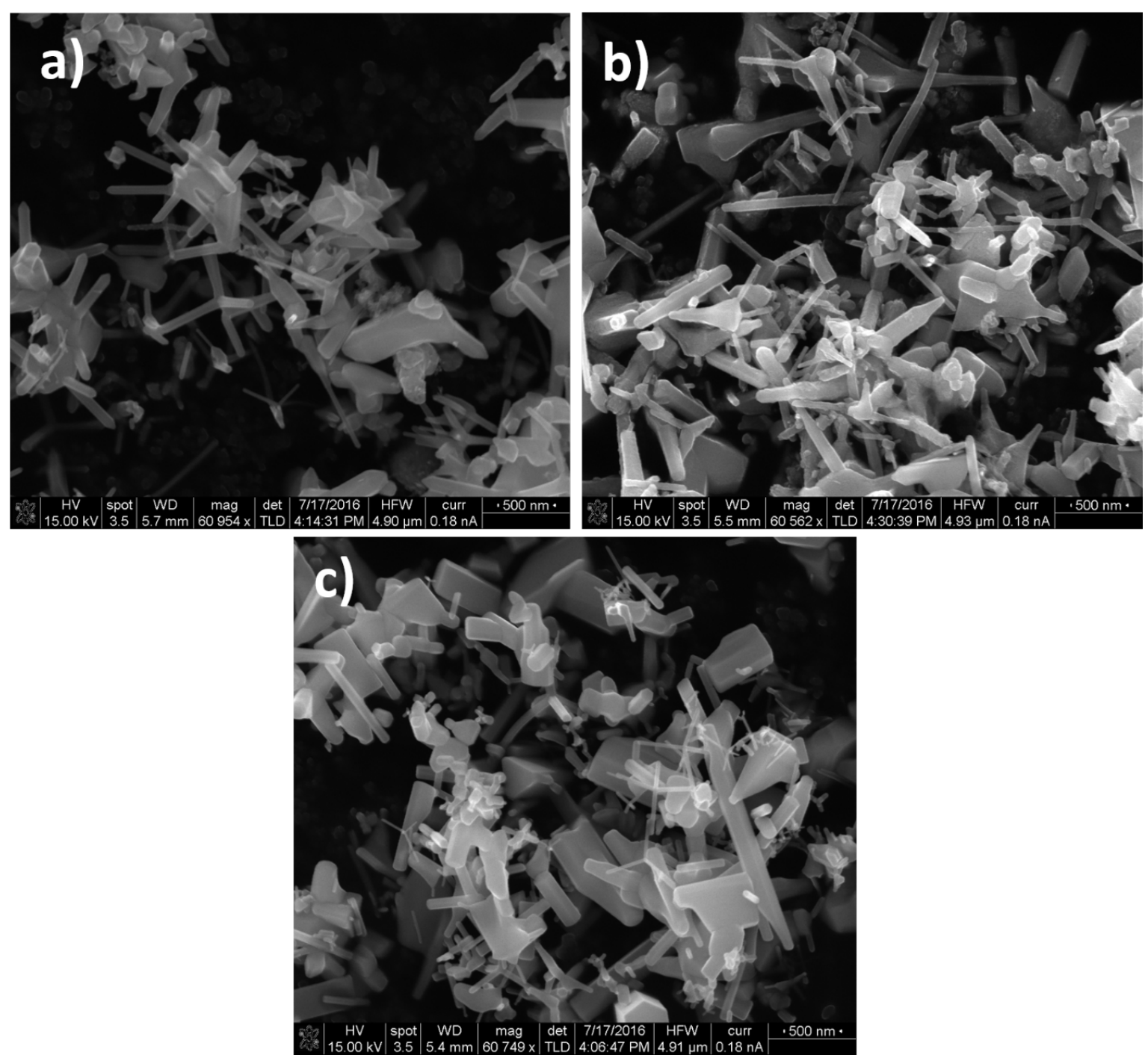

Figure 4.4.3: SEM images of zinc oxide nanowires produced using different oxygen flow rates: a) 15 LPM, b) 30 LPM, and c) 45 LPM 

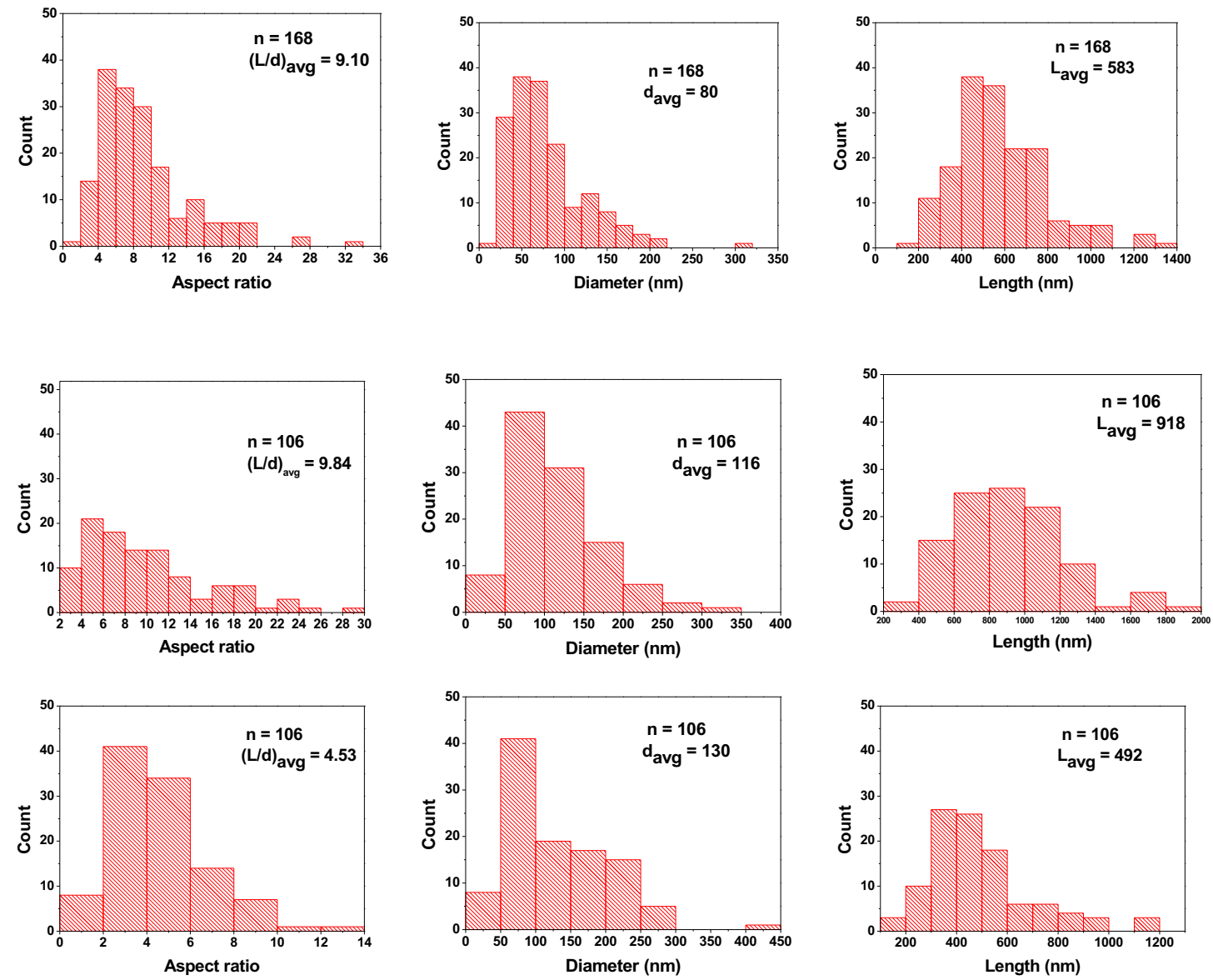

Figure 4.4.4: Effect of oxygen flow rate on nanowire morphology: first row - 15 LPM, second row - 30 LPM, and third row - 45 LPM

Figure 4.4.5 shows SEM images of zinc oxide nanowires produced using different propane flow rates. There was no big difference in the aspect ratio of nanowires produced using different propane flow rate. However, the nanowire diameter and length were increased with increasing propane flow rate. At 10 LPM of propane, the longest wires of $788 \mathrm{~nm}$ were obtained while the thinnest wires were obtained using 2 LPM of propane as shown in Figure 4.4.6. In addition, unreacted metal particles were observed at 2 LPM of propane. The low propane flow rate provides low flame volume and thus metal particles 
got unreacted.
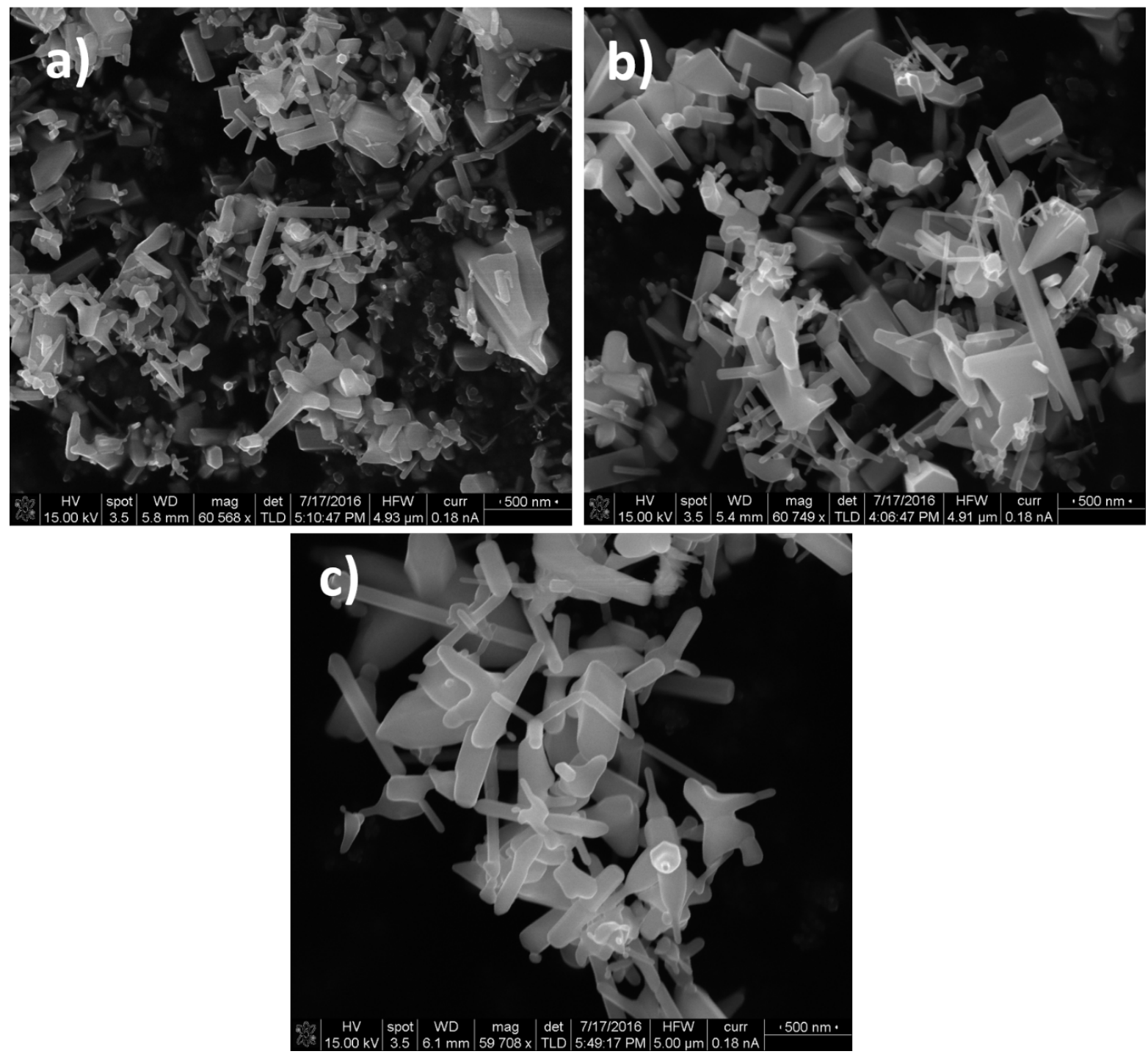

Figure 4.4.5: SEM images of zinc oxide nanowires produced using different propane flow rates: a) 2 LPM, b) 5 LPM, and c) 10 LPM
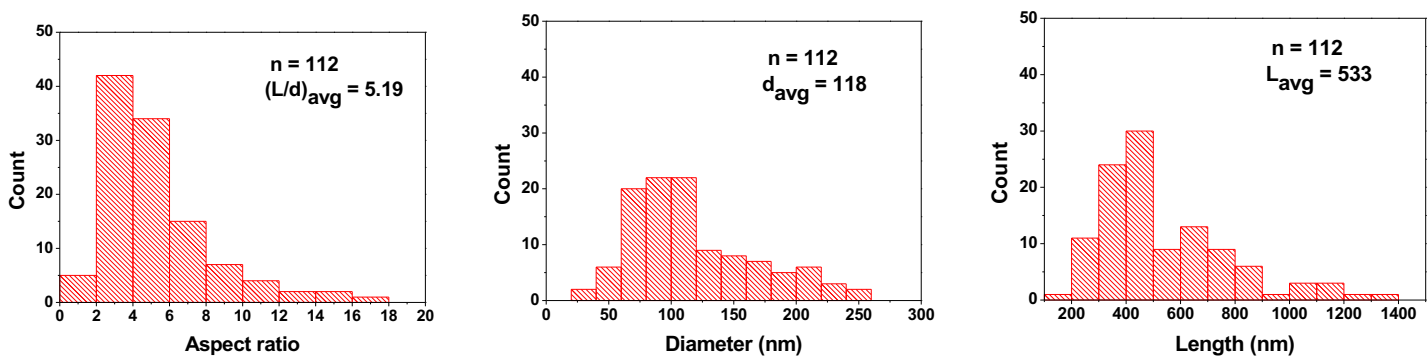

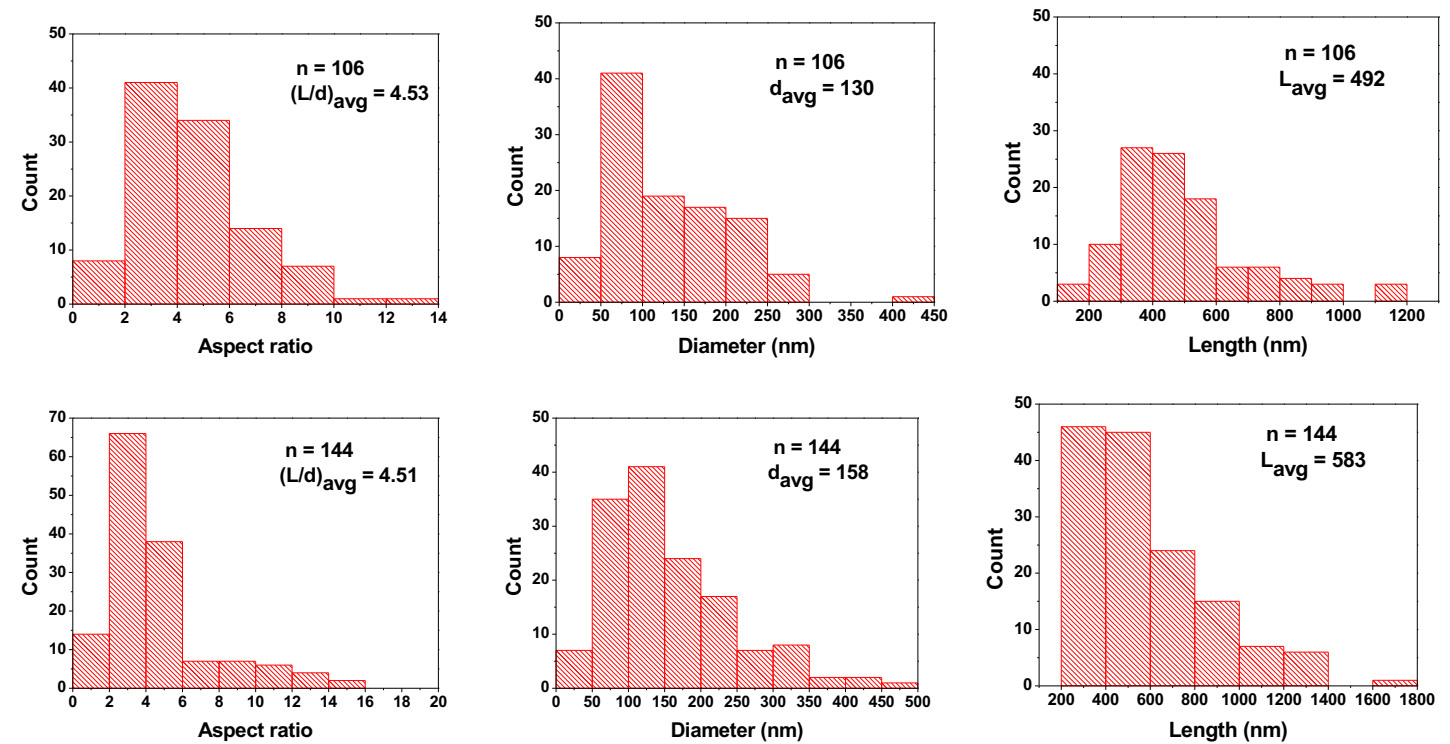

Figure 4.4.6: Effect of propane flow rate on nanowire morphology: first row of 2 LPM, second row of $5 \mathrm{LPM}$ gram/min, and third row of $10 \mathrm{LPM}$

\subsubsection{Effect of hydrocarbon flame}

It is important to investigate the effect of hydrocarbon source on the nanowire morphology because the different hydrocarbons have different carbon contents. The standard heat of combustion of propane $\left(\mathrm{C}_{3} \mathrm{H}_{8}-2220 \mathrm{~kJ} / \mathrm{mol}\right)$ is nearly $150 \%$ and $71 \%$ higher than that of methane $\left(\mathrm{CH}_{4}-889 \mathrm{~kJ} / \mathrm{mol}\right)$ and acetylene $\left(\mathrm{C}_{2} \mathrm{H}_{2}-1300 \mathrm{~kJ} / \mathrm{mol}\right)$ [87]. As expected, the resulting flame temperature were different. $\mathrm{C}_{3} \mathrm{H}_{8}$ has highest heat of combustion giving highest flame temperature while methane $\left(\mathrm{CH}_{4}\right)$ gives lowest flame temperature due to lowest heat of combustion. It is worth noting that the temperature of flame depends on the ratio of hydrocarbon and oxygen. In order to get the most accurate result, experiments were conducted at the ratio of oxygen to hydrocarbon as twice as stoichiometry of combustion reaction (Table 4.4.1). 
Table 4.4.1: Experiment condition for the study of effect of hydrocarbon

\begin{tabular}{|c|c|c|c|c|}
\hline No & Combustion reaction & Stoichiometry & $\begin{array}{c}\text { Hydrocarbon } \\
\text { flow rate (LPM) }\end{array}$ & $\begin{array}{c}\text { Oxygen flow } \\
\text { rate (LPM) }\end{array}$ \\
\hline 1 & $\mathrm{CH}_{4}+2 \mathrm{O}_{2} \rightarrow \mathrm{CO}_{2}+2 \mathrm{H}_{2} \mathrm{O}$ & $1: 2$ & 5 & 20 \\
\hline 2 & $\mathrm{C}_{3} \mathrm{H}_{8}+5 \mathrm{O}_{2} \rightarrow 3 \mathrm{CO}_{2}+4 \mathrm{H}_{2} \mathrm{O}$ & $1: 5$ & 5 & 50 \\
\hline 3 & $\mathrm{C}_{2} \mathrm{H}_{2}+2.5 \mathrm{O}_{2} \rightarrow 2 \mathrm{CO}_{2}+\mathrm{H}_{2} \mathrm{O}$ & $1: 2.5$ & 5 & 25 \\
\hline
\end{tabular}

Figure 4.4.7 shows SEM images of zinc oxide nanowires produced using different hydrocarbons. The statistic measurements are shown in Figure 4.4.8 and 4.4.9. The nanowire diameter produced using $\mathrm{CH}_{4}$ and $\mathrm{C}_{2} \mathrm{H}_{2}$ is significant smaller (47 nm and 59) than that of and $\mathrm{C}_{3} \mathrm{H}_{8}$. The nanowire produced using $\mathrm{CH}_{4}$ was shorter length of $396 \mathrm{~nm}$ than that of $\mathrm{C}_{3} \mathrm{H}_{8}$ and $\mathrm{C}_{2} \mathrm{H}_{2}$. The nanowire aspect ratio decreased in the order $\mathrm{CH}_{4}(8.43)$ to $\mathrm{C}_{2} \mathrm{H}_{2}$ (8.34) to $\mathrm{C}_{3} \mathrm{H}_{8}$ (3.78). The $\mathrm{C}_{3} \mathrm{H}_{8}$ probably creates too high temperature flame that vaporize the zinc metal into vapor phase. Zinc as vapor got oxidized and condensed as particles. The high temperature flame was reported to promote the nanoparticle growth by sintering and coagulation [68]. Therefore, nanowires produced using $\mathrm{C}_{3} \mathrm{H}_{8}$ showed a low aspect ratio and short wire. 

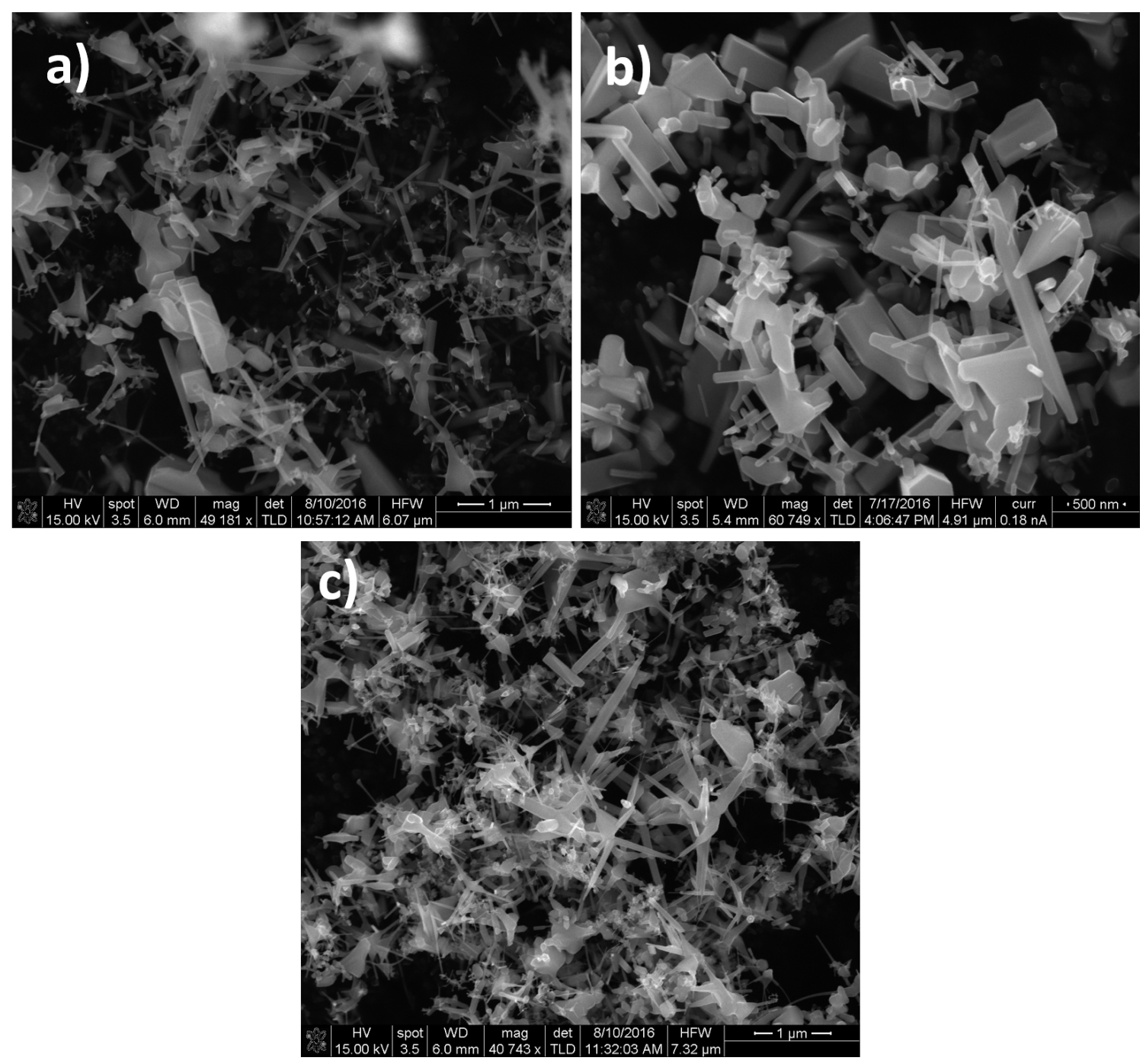

Figure 4.4.7: SEM images of zinc oxide nanowires produced using different hydrocarbons: a) methane, b) propane, and c) acetylene
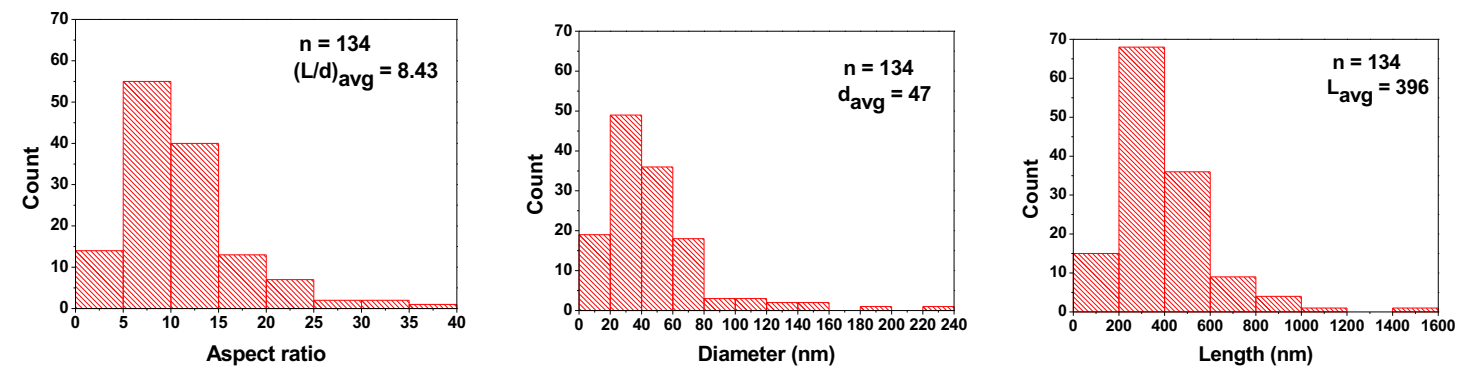

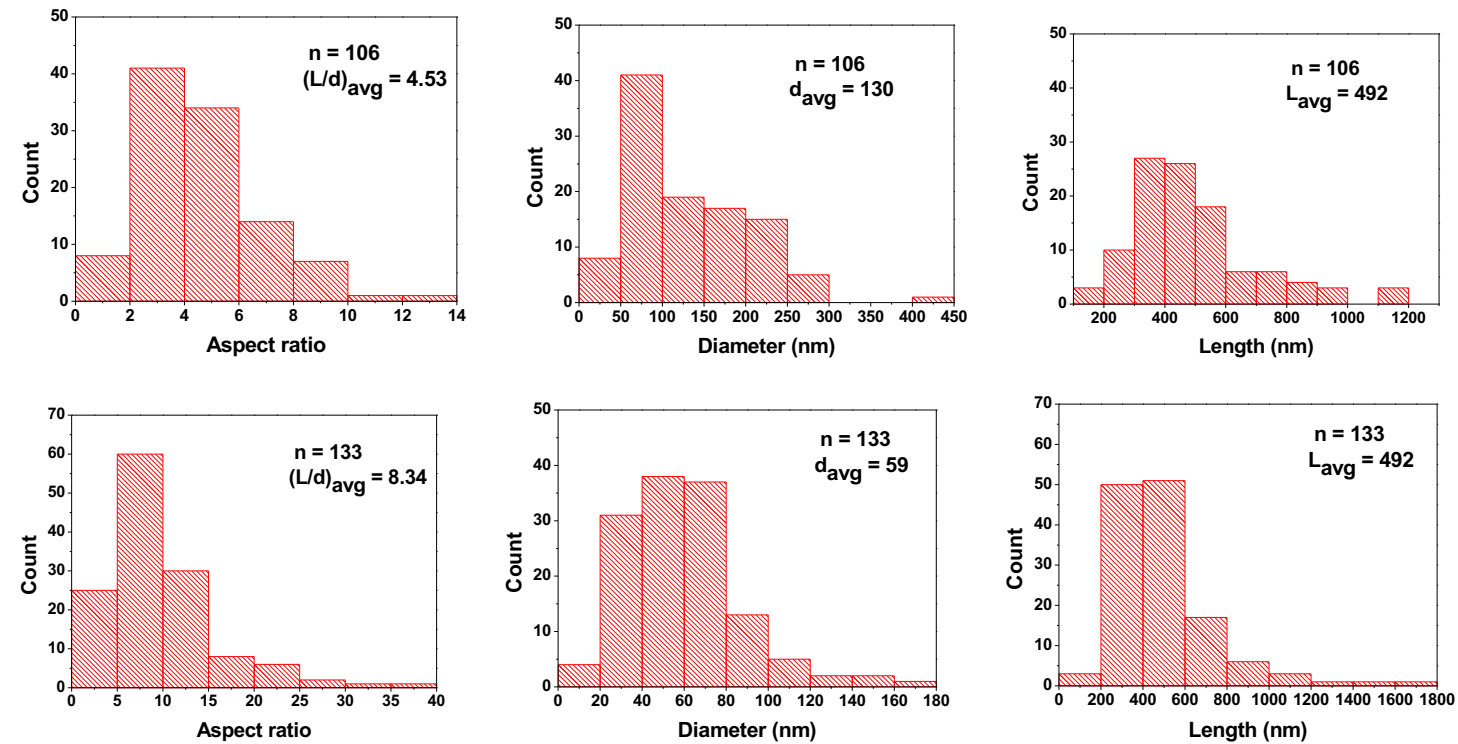

Figure 4.4.8: Effect of hydrocarbon type on nanowire morphology: first row of methane, second row of propane, and third row of acetylene

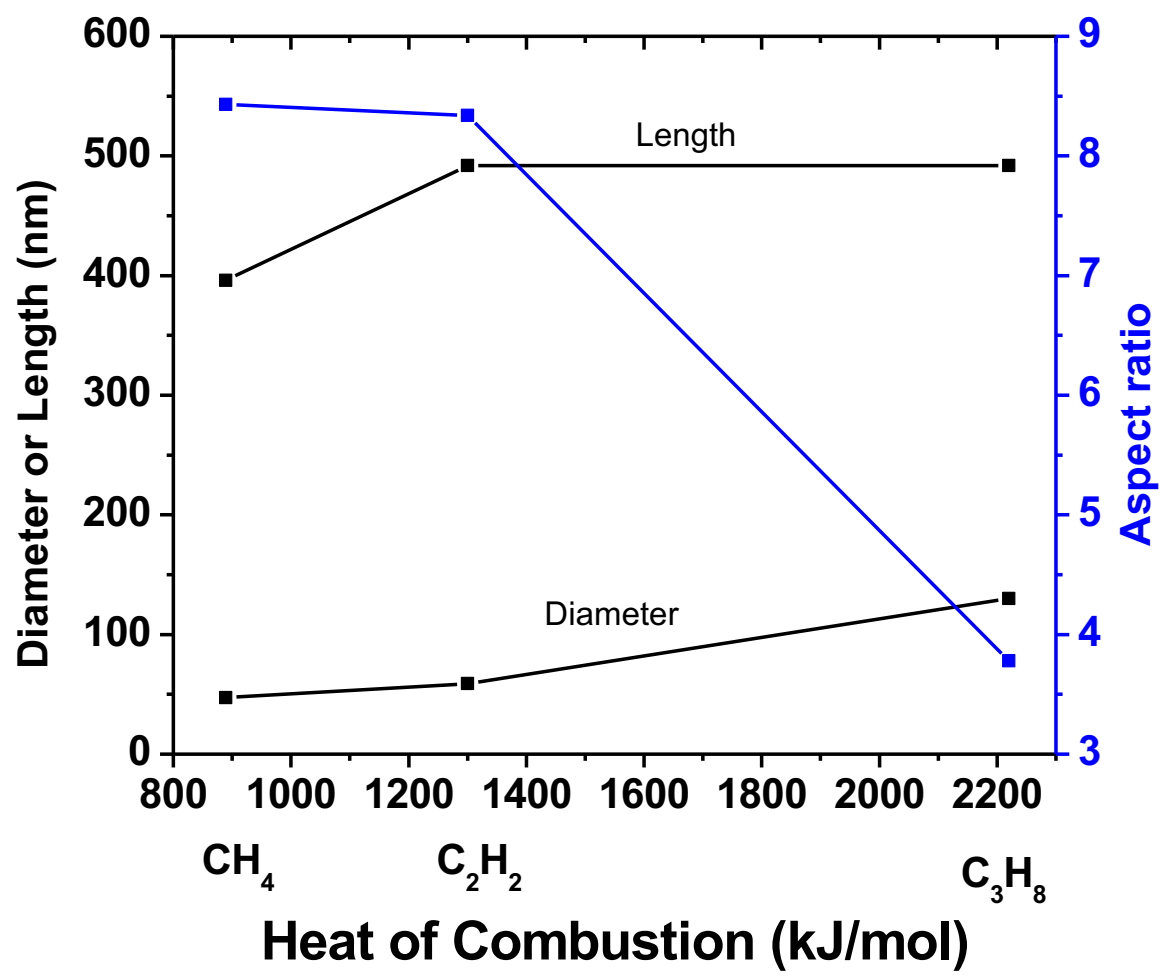


Figure 4.4.9: Effect of hydrocarbon type or heat of combustion on nanowire length and diameter

\subsubsection{Effect of feeding rate}

The feeding rate also was investigated for nanowire morphology control. Figure 4.4.10 shows SEM images of zinc oxide nanowires produced using different feeding rates There was no significant effect of feeding rate on aspect ratio and length. However, the nanowire diameter increased from 118 to $158 \mathrm{~nm}$ with increasing feeding rate from 3 to $24 \mathrm{grams} / \mathrm{min}$ as shown in Figure 4.4.11. Increasing the feeding rate has no significant effect on flame temperature and flame length, but influence on the supersaturation of zinc oxide on the surface of molten zinc particles, thereby controlling the diameter of nanowires. A similar increase in diameter with increasing of feeding rate has been shown for zirconia synthesis using spray flame pyrolysis[72]. Observation of unreacted zinc metal was seen at 24 grams $/ \mathrm{min}$. 

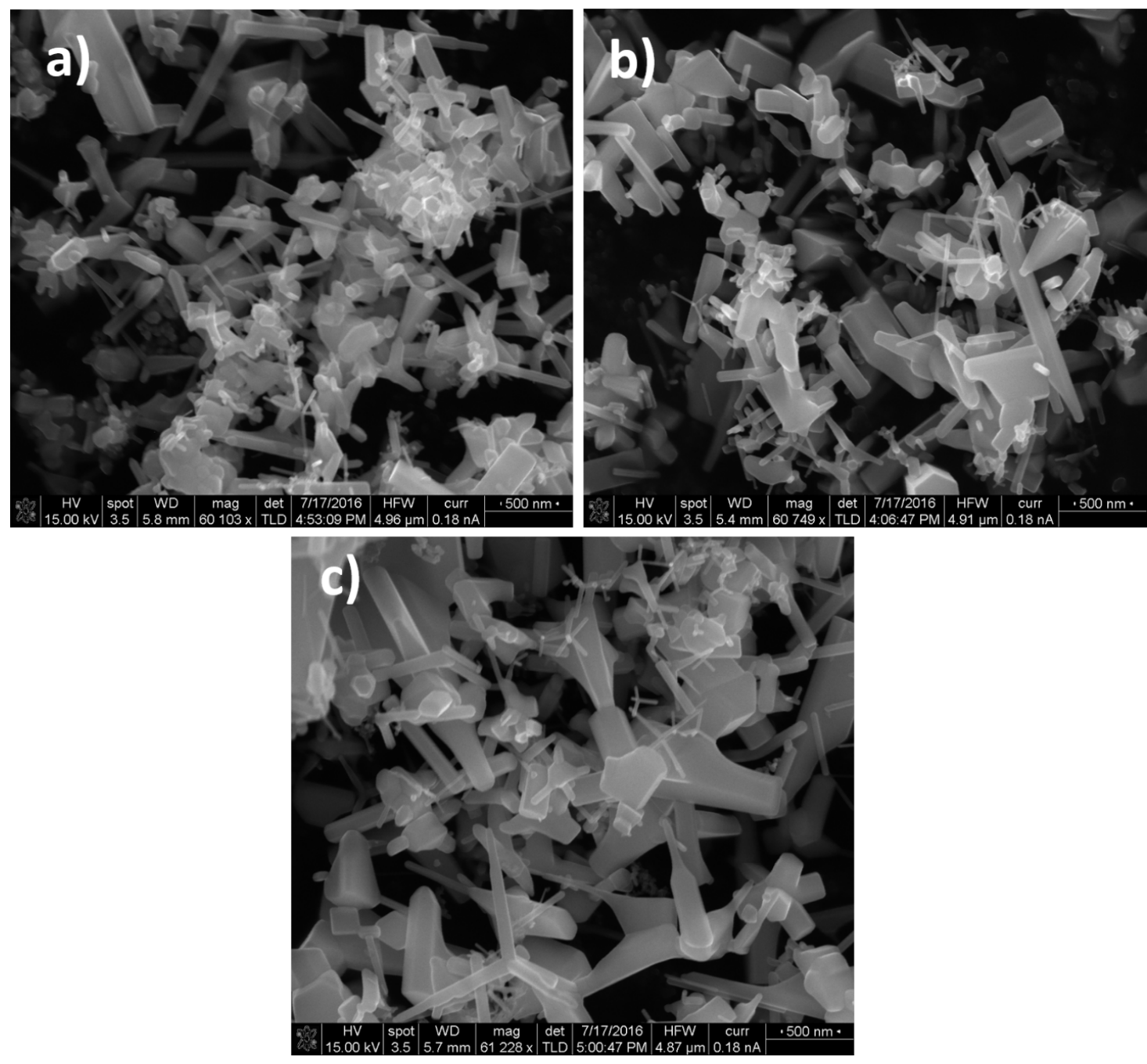

Figure 4.4.10: SEM images of zinc oxide nanowires produced using different feeding rates: a) 3 grams $/ \mathrm{min}$, b) 12 grams $/ \mathrm{min}$, and c) 24 grams $/ \mathrm{min}$
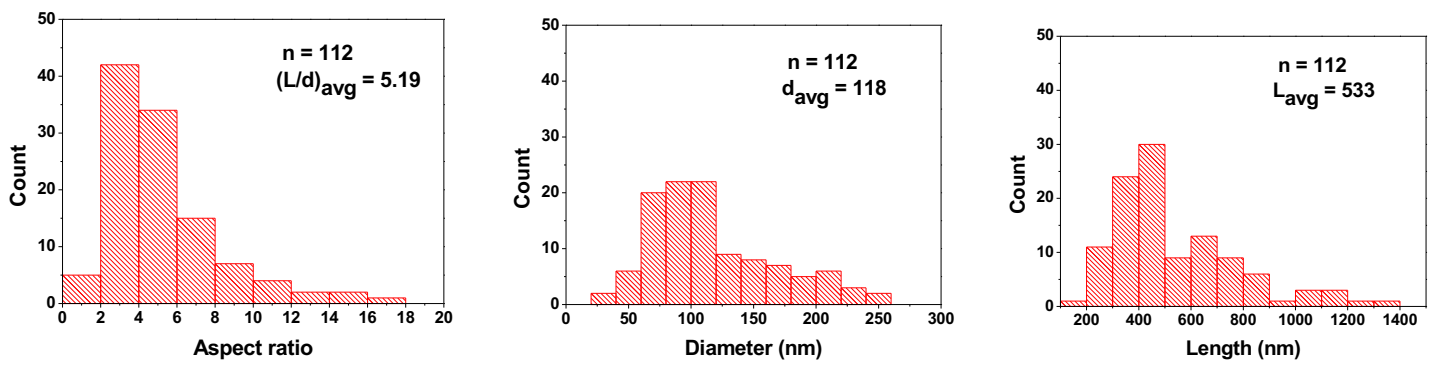

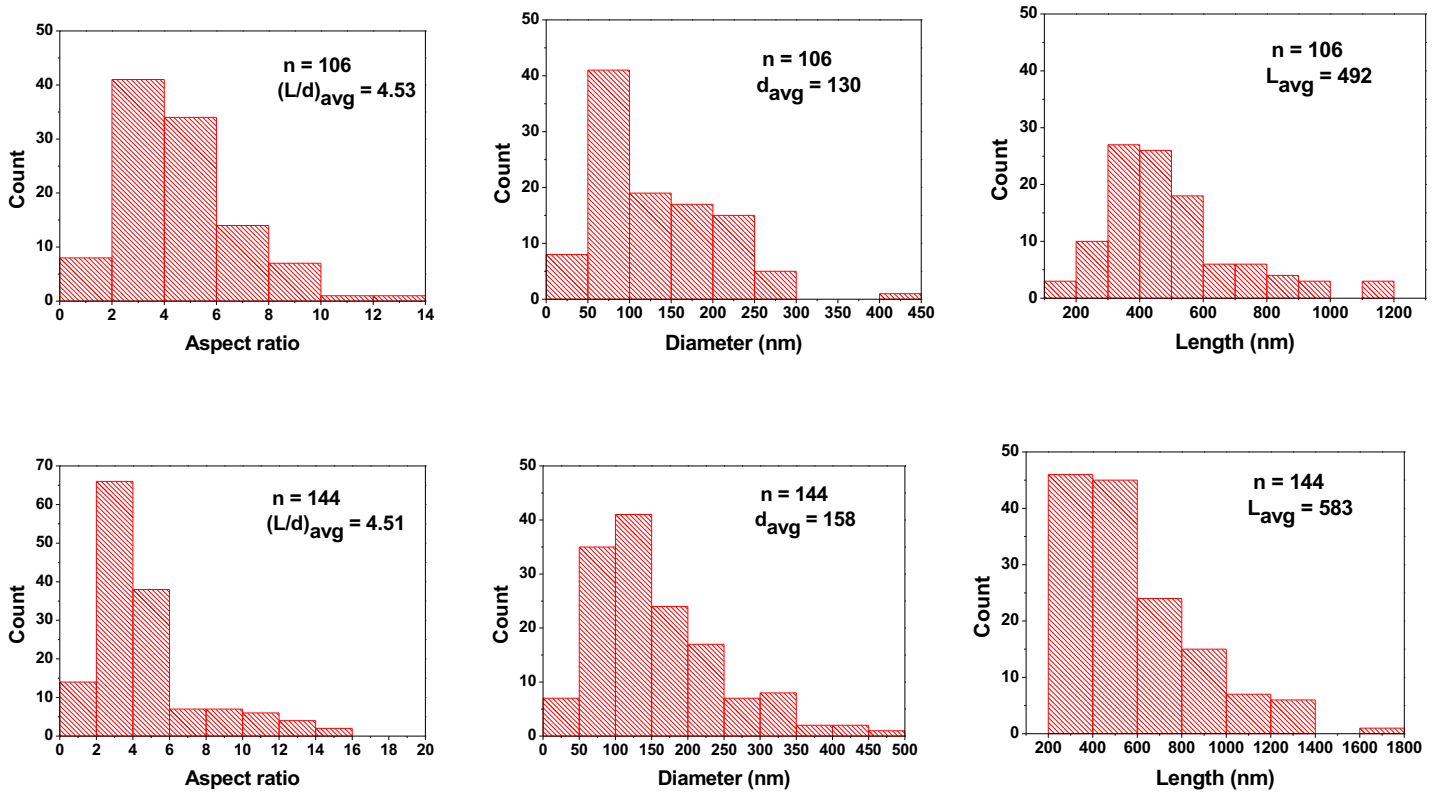

Figure 4.4.11: Effect of feeding rate on nanowire morphology: first row of 3 grams $/ \mathrm{min}$, second row of 12 grams/min, and third row of 24 grams $/ \mathrm{min}$

\subsubsection{Effect of flame volume or burner configuration}

Figure 4.4.12 shows a schematic of the custom-designed hydrocarbon burner for our fluidized bed reactor. The burner can work in a closed chamber at pressure of less than atmospheric pressure. The flame length can be controlled by varying the diameter of hydrocarbon tube and flow rate of hydrocarbon. The flame was about 13 using 0.06 in tube ID and hydrocarbon flow rate of 5 LPM (see Configuration A). The flame length increased to about 25 in using 0.17 tube ID and hydrocarbon flow rate of 10 LPM. The burner mouth was raised up to 9 in from the bottom of the fluidized bed reactor (see Configuration $\mathrm{B}$ and $\mathrm{C}$ ) 


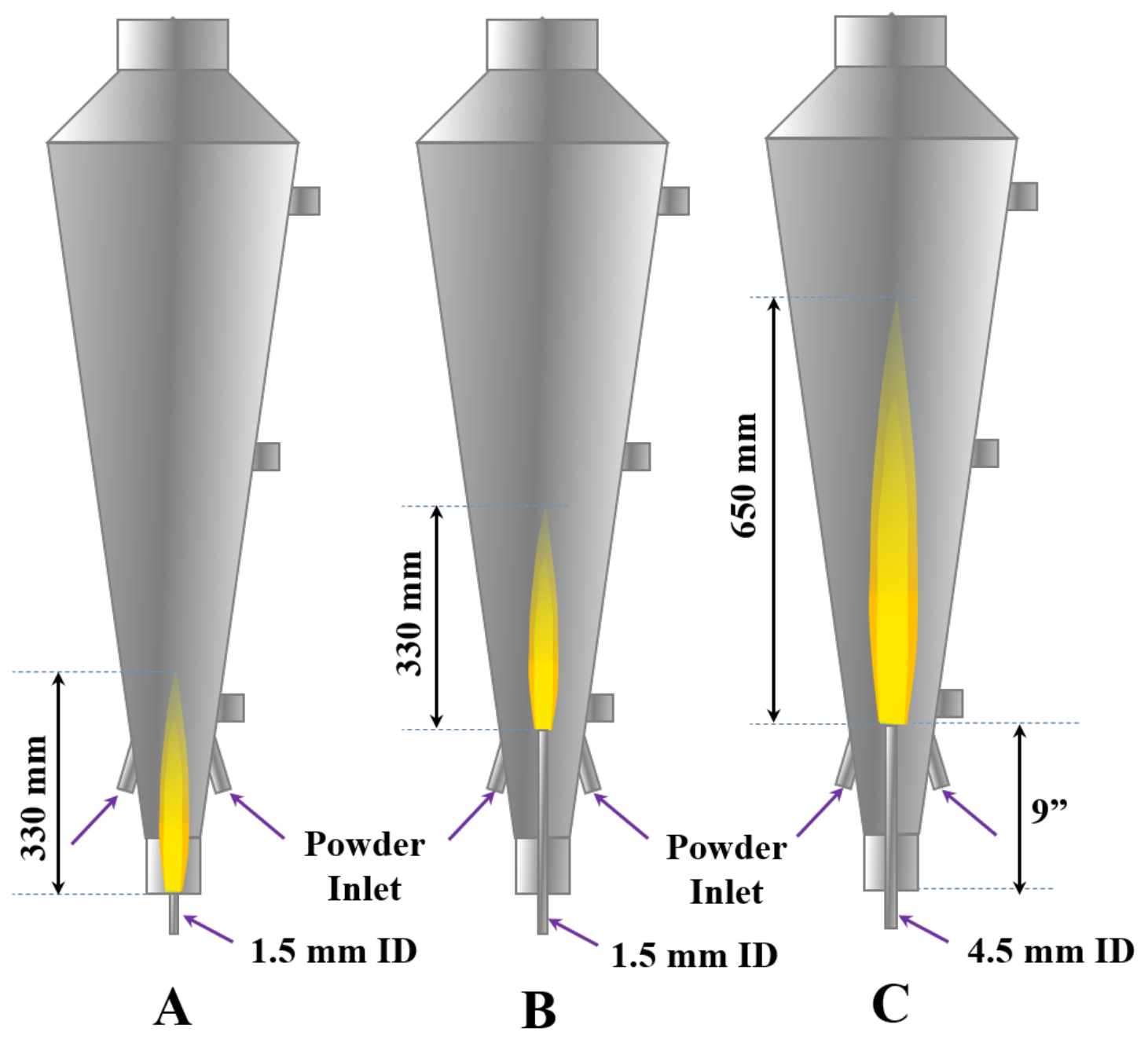

Figure 4.4.12: Schematic of burner configuration and corresponding flame length

Figure 4.4.13 shows the effect of hydrocarbon flame length burner configuration on the production rate and conversion. The conversion increased from about $30 \%$ to about $50 \%$ using the same flame length of $330 \mathrm{~mm}$. However, the burner has been raised up $23 \mathrm{~mm}$ so that there was much more effective flame volume for the reaction of zinc metal particles in flame. The conversion increased further up to about $90 \%$ using flame length of $650 \mathrm{~mm}$. Similarly, the production rate also increased with increasing flame length as shown in Figure 4.4.13. A highest production rate of $1.2 \mathrm{~kg} / \mathrm{hr}$ was obtained. 
Increasing flame volume would increase the number of metal particles involved in the reaction and therefore increasing the production rate. This is in agreement with two recent studies on nanoparticle production using different burner sizes $[88,89]$. As shown in Figure 4.4.14, a large quantity of nanowire powder of $30 \mathrm{~kg}$ was produced using fluidized bed reactor.

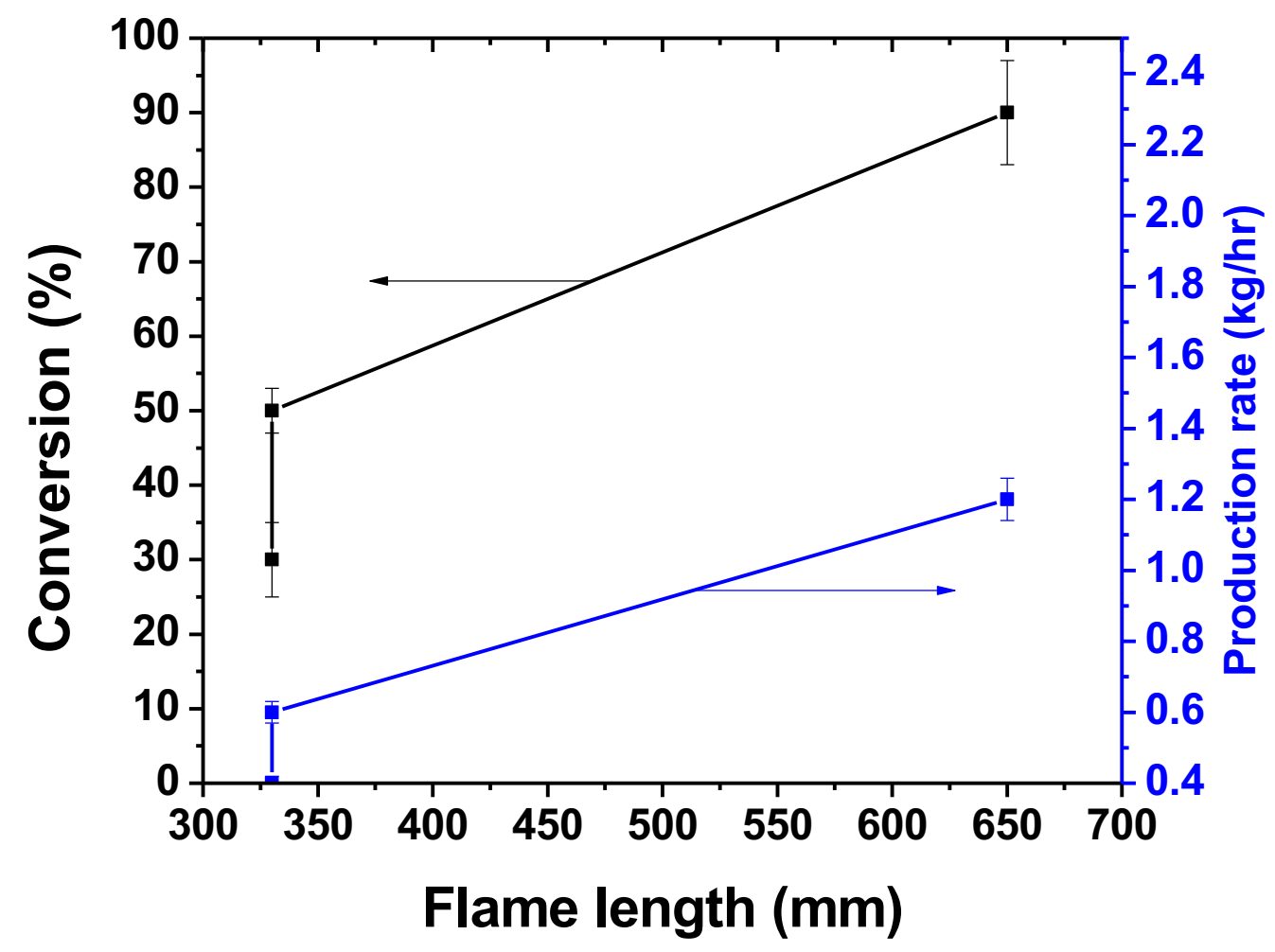

Figure 4.4.13: Effect of flame length on production rate and conversion 


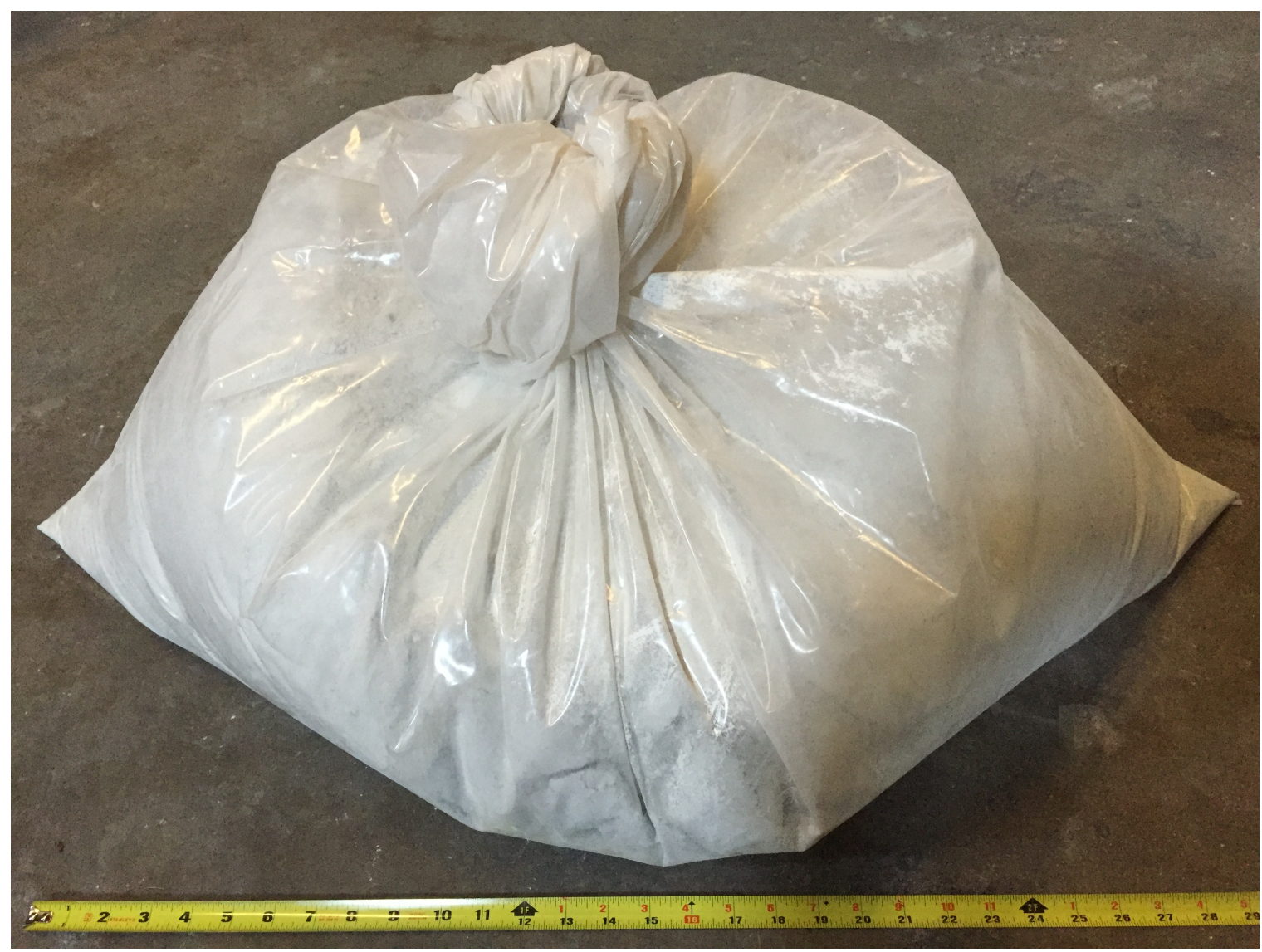

Figure 4.4.14: A photo of $30 \mathrm{~kg}$ of zinc nanowire powder produced using fluidized bed reactor

\subsection{Proof of fluidization}

It is important to demonstrate that the metal particles are being fluidizing inside the reactor chamber. To prove this, an experiment was performed where a small metal mesh is placed at the middle portion of the reactor through the second view port as shown in Figure 4.5.1. The reactor is operated using 5 LPM propane, 40 LPM oxygen, and 45 LPM air for dispersion. An argon flow of 8 LPM was used to deliver 5 grams of zinc metal powder per minute. The resulting powder sample was collected from both up and down side. 

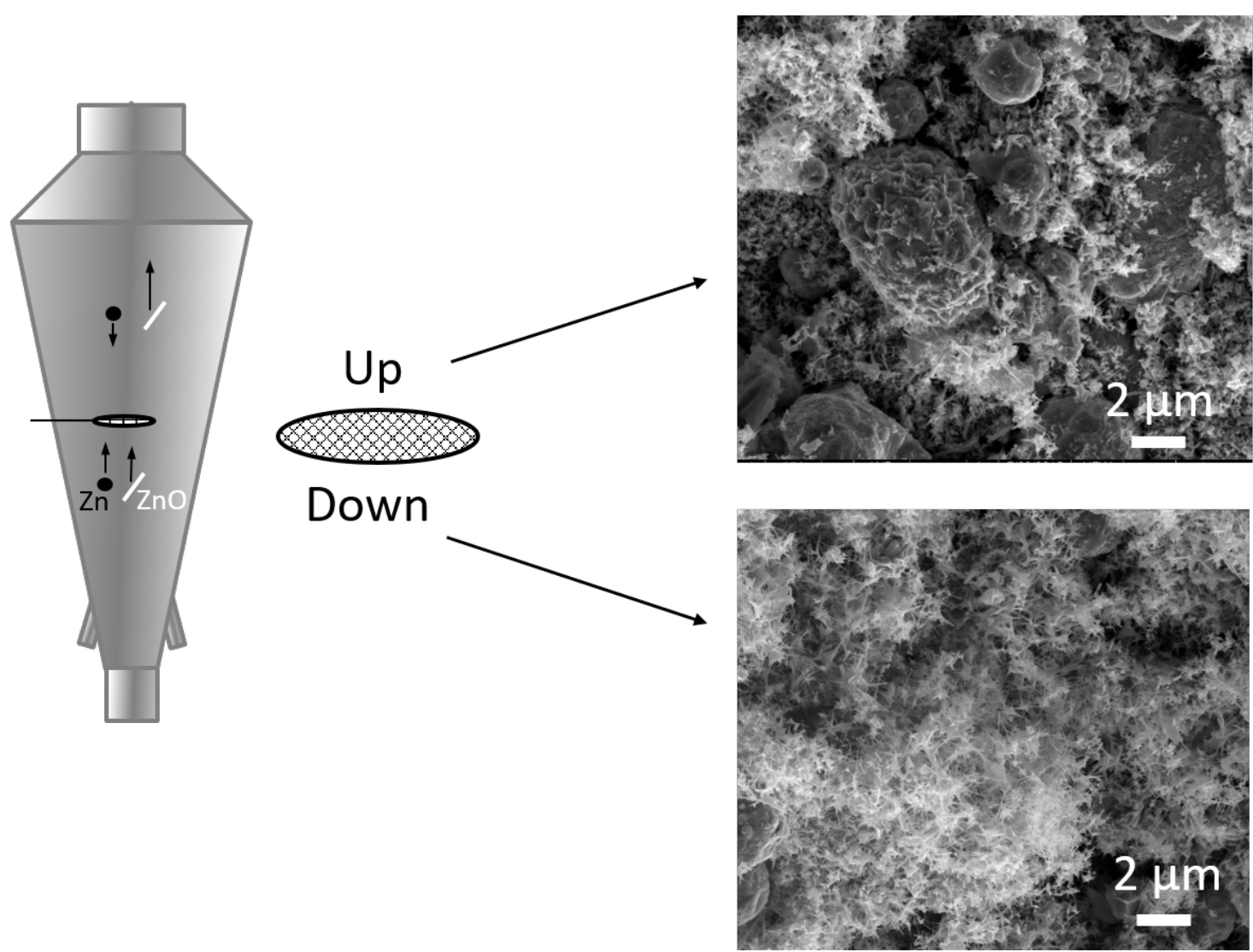

Figure 4.5.1: Schematic of the fluidized bed reactor with the metal mesh at the middle portion. The corresponding SEM images of zinc oxide powder collected on upside and downside of the metal mesh

A shown in Figure 4.5.1, the SEM images reveal that the sample collected on upside had a lot of unreacted zinc metal particles along with zinc oxide nanowires. The unreacted zinc particles were about 1-5 microns in diameter which is the same as the feeding zinc metal particles. Whereas, the sample collected on down side had only few unreacted metal particles of 1-2 microns in diameter. Moreover, the sample collected on upside was gray color which is slightly brighter than the color of zinc metal powder. The powder collected on down side was white color which is quite similar to the color of zinc oxide powder. This showed that the lighter zinc oxides tended to be blown upward and 
exit at the outlet of the reactor. While they were being blown upward, they hit the metal mesh and got stuck on the down side of the mesh. The heavier, larger zinc metal particles were also being blown up from the bottom. They were traveling upward along the reactor. However, due to the gravitational force outweighed the drag force due to the gas flow, the zinc metal particles tended to fall downward. While they were falling down, they settled on the upside of the metal mesh. The reason why there were few small zinc metal particles on the downside of the mesh is that the drag force exerted on them was still higher than the gravitation force exerted on them at the lower portion of the reactor.

\subsection{Zinc oxide as catalyst support for hydro-desulfurization of diesel}

\subsubsection{Introduction}

Reduction of sulfur in diesel fuel is important for environment as well as for the performance of catalytic converter in an automotive. Sulfur compounds in the fuels contribute to emissions of $\mathrm{SO}_{2}$ and sulfate particulate matter. Sulfur in transportation fuels degrades the catalytic converter resulting in increased $\mathrm{NO}_{\mathrm{x}}$ and $\mathrm{SO}_{\mathrm{x}}$ emissions which increase the potential for causing acid rain. Sulfur must be removed in any catalytic process because it poisons/deactivates the catalyst [90], which otherwise require frequent regeneration or replacement. Likewise, the performance of other catalytic devices such as fuel cells is also extremely sensitive to the presence of sulfur [91]. The sulfur irreversibly poisons noble metal catalysts present in the converter of an engine.

As of September 2007, all on-highway diesel fuel (ultra-low-sulfur diesel (ULSD)) sold at gas stations in the United States contains less than $15 \mathrm{ppm}$ of sulfur. The demand for ULSD has increased significantly in the last few years [83] because of continual reduction in sulfur limits in transportation fuels. As far as the gasoline is 
concerned, the Environmental Protection Agency (EPA) proposed a new regulation recently that would reduce its current sulfur content from 30 ppm to 10 ppm by 2017 [92] and much stricter limits are anticipated in future. Therefore, refiners in the United States and Europe have installed numerous new middle distillate hydrotreaters or revamped existing facilities. Governments in developing countries such as India, Mexico, and China are also imposing low sulfur regulations which has increased the demand for ultradeep desulfurization catalysts around the world [93].

Diesels and gasoline fuels contain a variety of heterocyclic sulfur compounds at few tens of ppm level which are difficult to remove using traditional hydro-sulfurization catalyst used today. For example, many catalysts undergo steric hindrance effects during adsorption with species such as 4-substituted and 4,6-disubstituted dibenzothiophenes. The refractory nature of b-DBTs comes from the steric hindrance around the sulfur atom $[83,94]$. The removal of these latter compounds presents a significant challenge to the industry [95-98]. The conventional hydro-desulfurization (HDS) process utilizes alumina- and silica-supported cobalt or nickel molybdenum catalyst and operate at 350$390^{\circ} \mathrm{C}$, and pressures between 60 and 90 bars [93]. Currently, for ultra-low sulfur diesel (ULSD) production, hydrotreating catalysts include Nickel-Molybdenum-Sulfur (Ni-Mo$\mathrm{S})$ or Cobalt-Molybdenum-Sulfur (Co-Mo-S) supported on $\gamma$-alumina $\left(\mathrm{Al}_{2} \mathrm{O}_{3}\right)$ [83]. However, the above catalysts suffer from low activity for heterocyclic compounds present below $50 \mathrm{ppm}$ and require higher temperature and pressure and/or low space velocities to bring sulfur down below $15 \mathrm{ppm}[99,100]$. At higher temperatures, there are some inherent issues that reduce the catalyst activity, and they include carbon deposition on catalyst surface and change of catalyst surface itself (re-organization of surface) [100, 
101] Hence, there is a crucial need to design high activity catalysts for ultra-deep desulfurization at moderate process conditions.

Here, we show the feasibility of using nickel nanoparticles supported on the zinc oxide nanowire surfaces as catalysts. Nickel can catalyze the hydrogenation reaction as well as the cleaving of cyclic sulfur compounds like thiophenes. The use of $\mathrm{ZnO}$ follows that of reactive adsorption concept in which sulfur from $\mathrm{Ni}$ clusters transfer to $\mathrm{ZnO}$ nanowire support [102-106]. Tawara's group [103] was the first to report the performance of a $\mathrm{Ni} / \mathrm{ZnO}$ catalyst as an adsorptive HDS catalyst for the kerosene toward kerosene-fed fuel cell applications.

Active nickel sites could be maintained until the $\mathrm{ZnO}$ nanowire supports get sulfide fully. ConocoPhillips developed a sulfur removal technology for gasoline, based on reactive adsorption that uses metal supported on $\mathrm{ZnO}$.

The use of nanowire support has multi-fold advantages for catalyst design. Some of them are highlighted here: 1) the single crystal nature and faceting improves the properties of the supported catalytically-active metals through the resulting nickel cluster morphology; 2) ability to produce well-defined surface facets therefore higher concentration of desired sites[12];2) potentially enhance metal-support interactions; 3) nanowire support undergo less sintering than conventional supports [107, 108]; and 4) nanoparticle on nanowire morphology presents an easier diffusion pathway for sulfur transfer from supported clusters to underlying support to maintain a high surface concentration of active nickel sites for deep desulfurization activity.

Even though, the nanowire supports are predicted to have the above-mentioned advantages, there are no studies for both deep HDS and aromatic hydrogenation using 
catalysts made from nanowire powders. Here, we report the ultra-deep HDS activity of nickel-rich metal cluster supported on $\mathrm{ZnO}$ NWs (Figure 4.6.1). The activity is tested using a variety of fuels and most importantly with diesel that contains most-refractive sulfur compounds such as 4,6-Dimethyldibenzothiophene (DMDBT). The nature of active catalyst clusters, uniformity of morphology, morphological stability, and activity of fresh and regenerated catalysts, are of fundamental interest.

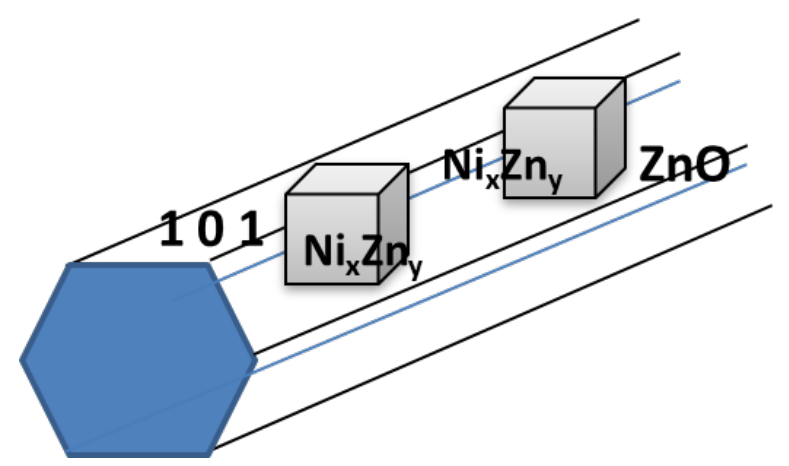

Figure 4.6.1: A schematic showing uniform morphologies for nanoclusters produced on mono-crystalline facets of c-plane oriented $\mathrm{ZnO}$ nanowire.

\subsubsection{Results and discussion}

\subsubsection{Textural Properties of Catalysts}

Table 4.6.2 summarizes the surface area and $\mathrm{NiO}$ particle sizes for some of the catalyst samples used in the desulfurization tests. In the catalyst samples A-F, the amount of Ni was varied from 12 to $30 \mathrm{wt} \%$. The BET surface areas were as high as $92.7 \mathrm{~m}^{2} / \mathrm{g}$ for $12 \% \mathrm{Ni}$ catalyst and as low as $8.5 \mathrm{~m}^{2} / \mathrm{g}$ for catalyst $17 \% \mathrm{Ni}$ catalyst without $\gamma-\mathrm{Al}_{2} \mathrm{O}_{3}$. This indicates that $\gamma-\mathrm{Al}_{2} \mathrm{O}_{3}$ helps in increasing the surface of the catalyst because of its higher surface area than nanowires. The metal/oxide can also nucleate on $\gamma-\mathrm{Al}_{2} \mathrm{O}_{3}$. The alumina also helps in binding the constituents of the catalyst by imparting mechanical 
strength to it [83]. The catalyst extrudates without $\gamma-\mathrm{Al}_{2} \mathrm{O}_{3}$ became powder after hydrodesulfurization reaction whereas with $\gamma-\mathrm{Al}_{2} \mathrm{O}_{3}$ the extrudates retained its shape and size.

Table 4.6.2: Catalysts- composition, BET surface area and crystallite size

\begin{tabular}{|c|c|c|c|c|c|}
\hline Catalysts & $\begin{array}{l}\mathrm{Ni} \\
(\mathrm{wt} \%)\end{array}$ & $\begin{array}{l}\mathrm{ZnO} \\
(\mathrm{wt} \%)\end{array}$ & $\begin{array}{l}\gamma-\mathrm{Al}_{2} \mathrm{O}_{3} \\
(\mathrm{wt} \%)\end{array}$ & $\operatorname{BET}\left(\mathrm{m}^{2} / \mathrm{g}\right)$ & $\begin{array}{l}\text { Crystallite } \\
\text { Size-NiO (nm) }\end{array}$ \\
\hline A & 17 & 83 & -- & 8.5 & 25.1 \\
\hline B & -- & 41.6 & 58.4 & 123.0 & -- \\
\hline C & 12 & 58.7 & 11.3 & 92.7 & 16.0 \\
\hline $\mathrm{D}$ & 15 & 58.7 & 26.3 & 78.7 & 10.2 \\
\hline E & 21 & 58.7 & 20.3 & 76.9 & 11.0 \\
\hline \multirow[t]{2}{*}{$\mathrm{F}$} & 30 & 58.7 & 11.3 & 46.2 & 18.9 \\
\hline & $\begin{array}{r}\text { Mo } \\
\text { (wt\%) }\end{array}$ & & & & \\
\hline G & 123 & 58.7 & 26.3 & 59.8 & 22.0 \\
\hline
\end{tabular}

In general, the crystallite size decreases after addition of $\gamma-\mathrm{Al}_{2} \mathrm{O}_{3}$ as shown in Table 4.6.2. The catalyst without $\gamma-\mathrm{Al}_{2} \mathrm{O}_{3}$ has $\mathrm{NiO}$ crystallite size of about $25 \mathrm{~nm}$ whereas with $\gamma-\mathrm{Al}_{2} \mathrm{O}_{3}$, the size range for $\mathrm{NiO}$ crystallites is from 11 to $16 \mathrm{~nm}$. However, addition of Mo increases the crystallite size to $22 \mathrm{~nm}$ (Sample G). This is only a preliminary observation and cannot be concluded as a phenomenon. Optimization of active nickel loading is crucial. High content of $\mathrm{Ni}$ and low amount of $\mathrm{ZnO}$ will provide higher activity initially but will degrade quickly due to low amount of interfacial area between $\mathrm{Ni}$ and $\mathrm{ZnO}$. Therefore, in order to obtain long life and high activity, a balance between the compositions of $\mathrm{Ni}$ and $\mathrm{ZnO}$ is required [103]. 


\subsubsection{Ultra-deep Desulfurization Activity Testing of Catalysts}

The ultra-deep desulfurization activity of the catalyst samples from Table 4.6.2 was tested using the packed bed reactor using a temperature of $290^{\circ} \mathrm{C}$, Pressure of $30 \mathrm{bar}$, and liquid hourly space velocity (LHSV) values ranging from 1-2.2 $\mathrm{h}^{-1}$. In all cases, the data shows $99 \%$ removal of sulfur from feed streams at the moderate process conditions indicating high activity of our nickel supported on $\mathrm{ZnO}$ nanowires. Tests were stopped after pre-set times ranging from 100 - 150 hours. In Figure 4.6.2, using our nickel supported on $\mathrm{ZnO}$ nanowire catalysts, with a diesel feed (DF2) containing $196 \mathrm{ppm}$ of total sulfur, the sulfur content in the treated diesel was less than $1 \mathrm{ppm}$. And testing of desulfurization using diesel feed (DF1) containing $30 \mathrm{ppm}$ of sulfur using catalyst containing only $\mathrm{ZnO} / \mathrm{Al}_{2} \mathrm{O}_{3}$ showed sulfur breakthrough in less than $30 \mathrm{~h}$ and losing all of the activity in 50 hours. The $\mathrm{ZnO} / \mathrm{Al}_{2} \mathrm{O}_{3}$ catalyst showed some activity to reduce sulfur level in the first few hours but the activity was not significant enough to remove sulfur compounds (4,6-Dimethyldibenzothiophene and 4-Methyldibenzothiophene). The lack of activity with $\mathrm{ZnO}$ and $\gamma-\mathrm{Al}_{2} \mathrm{O}_{3}$ proves that either $\mathrm{Ni}$ or its alloys are required to provide the necessary catalytic activity. As shown in Figure 4.6.2, all other catalysts with Ni loading on $\mathrm{ZnO} \mathrm{NW}$ powder along with $\gamma-\mathrm{Al}_{2} \mathrm{O}_{3}$ showed ultra-deep desulfurization to levels less than 1 ppm with steady state activity demonstrated for 100-150 hrs. 


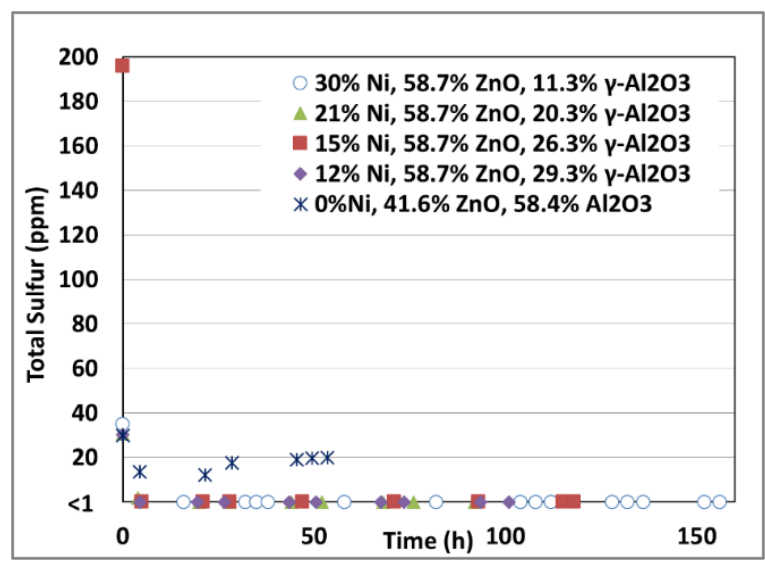

Figure 4.6.2: Sulfur content in diesel samples at different times-on-stream: Comparison of catalysts using different $\mathrm{Ni}$ loading amounts on $\mathrm{ZnO} \mathrm{NW}$

Figure 4.6.3 shows the photographs of treated diesel samples as a function of time. The catalyst was $12 \% \mathrm{Ni}, 58.7 \% \mathrm{ZnO}, 29.3 \% \gamma-\mathrm{Al}_{2} \mathrm{O}_{3}$. The change in color is probably due to aromatic saturation. Just after few hours, the diesel sample was colorless due to very low amount of aromatics and then started picking up color after $60 \mathrm{hrs}$ and then remained almost the same thereafter. Thus lighter color is indicative of better diesel quality.

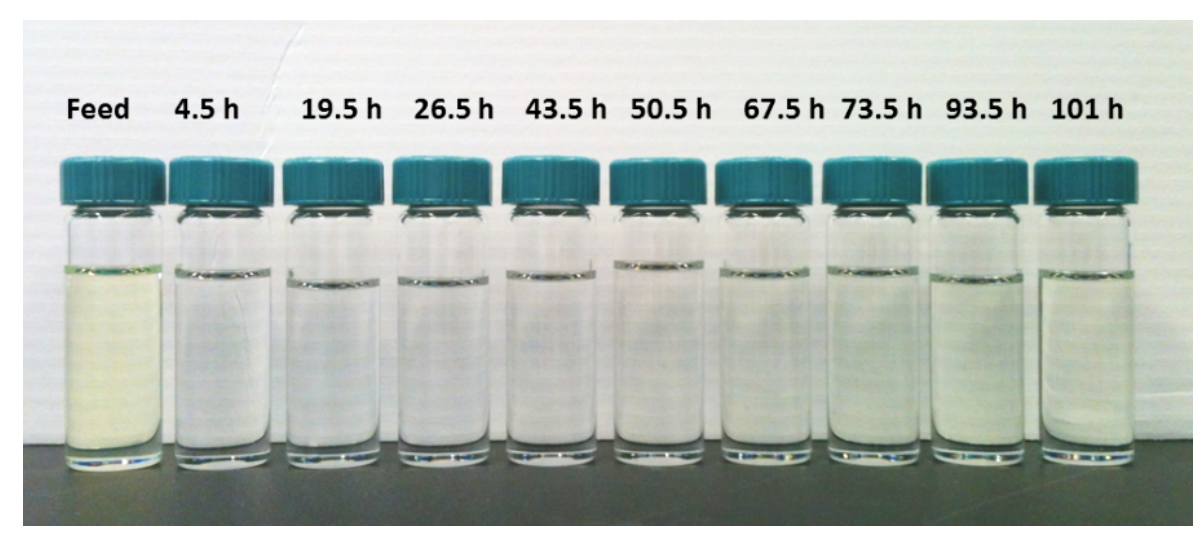

Figure 4.6.3: Color of diesel samples with time 


\subsubsection{Discussion on Activity and Mechanism}

In order to understand the nature and composition of catalyst site and the sulfur transfer pathway, the spent catalysts are characterized using XRD, and TEM after testing. Figure 4.6.4 shows SEM images for fresh and spent versions of a catalyst containing 15\% $\mathrm{Ni}, 58.7 \% \mathrm{ZnO}, 26.3 \% \gamma-\mathrm{Al}_{2} \mathrm{O}_{3}$. The diameters and lengths of NWs were $70-150 \mathrm{~nm}$ and $250 \mathrm{~nm}-2 \mu \mathrm{m}$, respectively. The spent catalyst was used to desulfurize the DF2 feed containing various sulfur compounds (Table 4.6.1) over $110 \mathrm{~h}$. It is clear from the SEM images that the nanowire morphology was retained after reaction. This is highly critical for good activity and selectivity of the catalyst. Figure 4.6 .4 shows the XRD patterns for fresh and spent catalysts containing $15 \% \mathrm{Ni}, 58.7 \% \mathrm{ZnO}, 26.3 \% \gamma-\mathrm{Al}_{2} \mathrm{O}_{3}$. In general, the data shows the presence of $\mathrm{NiO}$ and $\mathrm{ZnO}$ phases in all the fresh catalysts. There was no shift observed in $\mathrm{ZnO}$ peaks. Upon reduction step, $\mathrm{NiO}$ clusters reduce to either nickel or nickel rich nickel-zinc compound. The spent catalyst shows $\mathrm{ZnS}$ but no NiS phase which supports the reactive adsorption mechanism in which active metal sites are preserved for ultra-deep desulfurization. The absence of NiS phase indicates that the sulfur from sulfided $\mathrm{Ni}$ or $\mathrm{Ni}$ rich clusters is transferred to underlying $\mathrm{ZnO}$ nanowire supports effectively. 

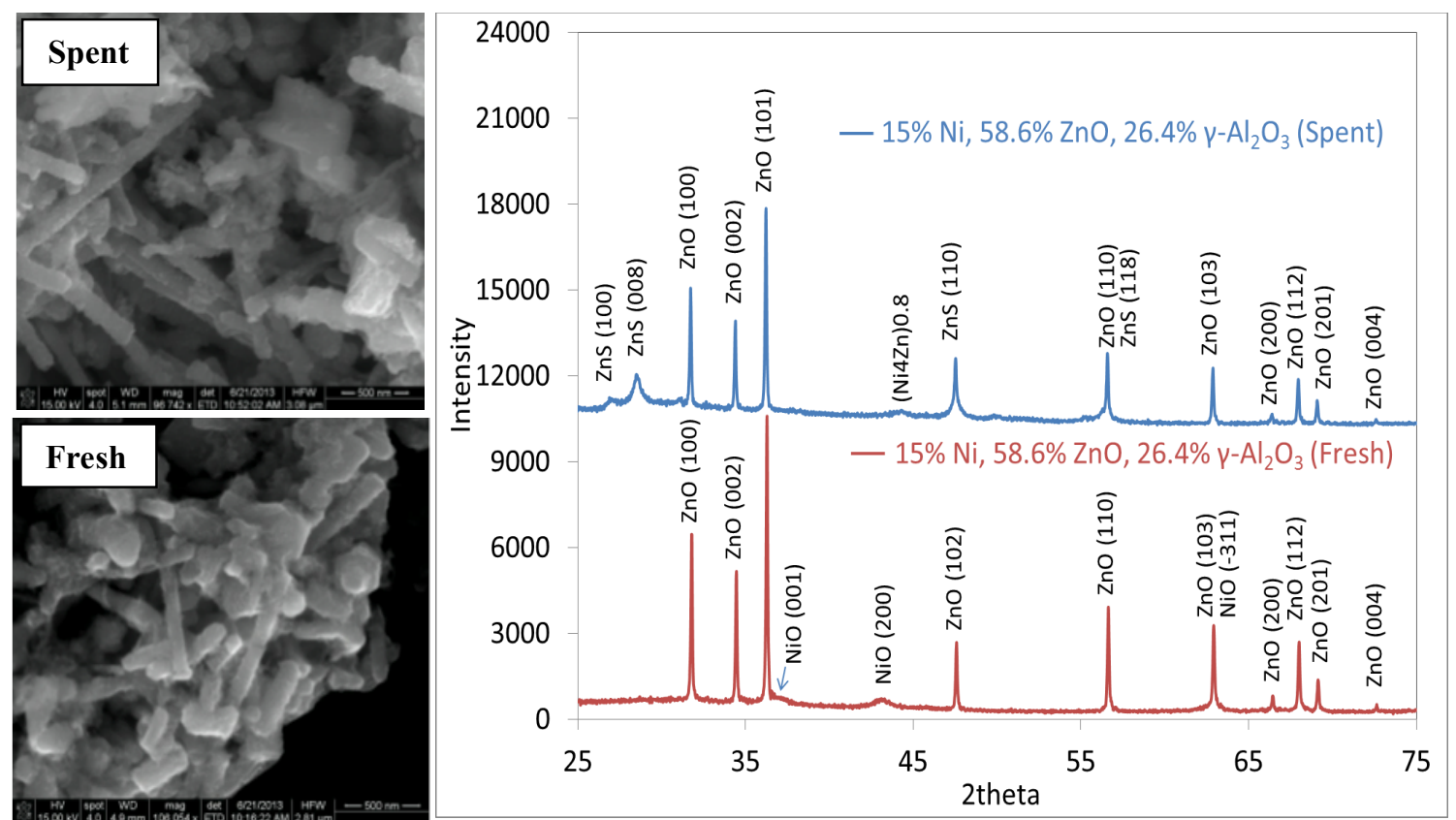

Figure 4.6.4: SEM micrographs and XRD patterns of fresh and spent catalyst $15 \% \mathrm{Ni}$, $58.7 \% \mathrm{ZnO}, 26.3 \% \gamma-\mathrm{A} 12 \mathrm{O} 3$

Figure 4.6.5 (a) shows the XRD patterns for fresh and spent catalysts containing $12 \% \mathrm{Ni}, 58.7 \% \mathrm{ZnO}, 29.3 \% \gamma-\mathrm{Al}_{2} \mathrm{O}_{3}$. The same characteristics were observed as $15 \% \mathrm{Ni}$ discussed before. It is important to note that, although $15 \%$ catalyst and $12 \% \mathrm{Ni}$ catalyst faced very different amounts of sulfur in the feeds still there is no significant difference in their XRD features except for nickel-zinc alloy. In the present case, it is $\mathrm{Ni}_{0.7} \mathrm{Zn}_{0.3}$, whereas in the earlier one it was $\left(\mathrm{Ni}_{4} \mathrm{Zn}\right)_{0.8}$, both are rich in nickel. It is highly likely that the nickel rich $\mathrm{Ni}_{1-\mathrm{x}} \mathrm{Zn}_{\mathrm{x}}$ alloy clusters are formed during reduction step. It has been shown earlier that reduction at $430^{\circ} \mathrm{C}$ enables the formation of nickel alloy with zinc [105]. Figure 4.6.5(b) shows the XRD patterns for $12 \% \mathrm{Ni}, 3 \% \mathrm{Mo}, 58.7 \% \mathrm{ZnO}, 26.3 \% \gamma-$ $\mathrm{Al}_{2} \mathrm{O}_{3}$. This catalyst contained $\mathrm{MoNi}, \mathrm{NiO}$, and $\mathrm{ZnO}$ phases. The spent catalyst had MoNi, NiO, $\mathrm{ZnO}$ and $\mathrm{ZnS}$ phases. This catalyst also did not show any NiS phase which reaffirms our hypothesis of reactive adsorption mechanism. 

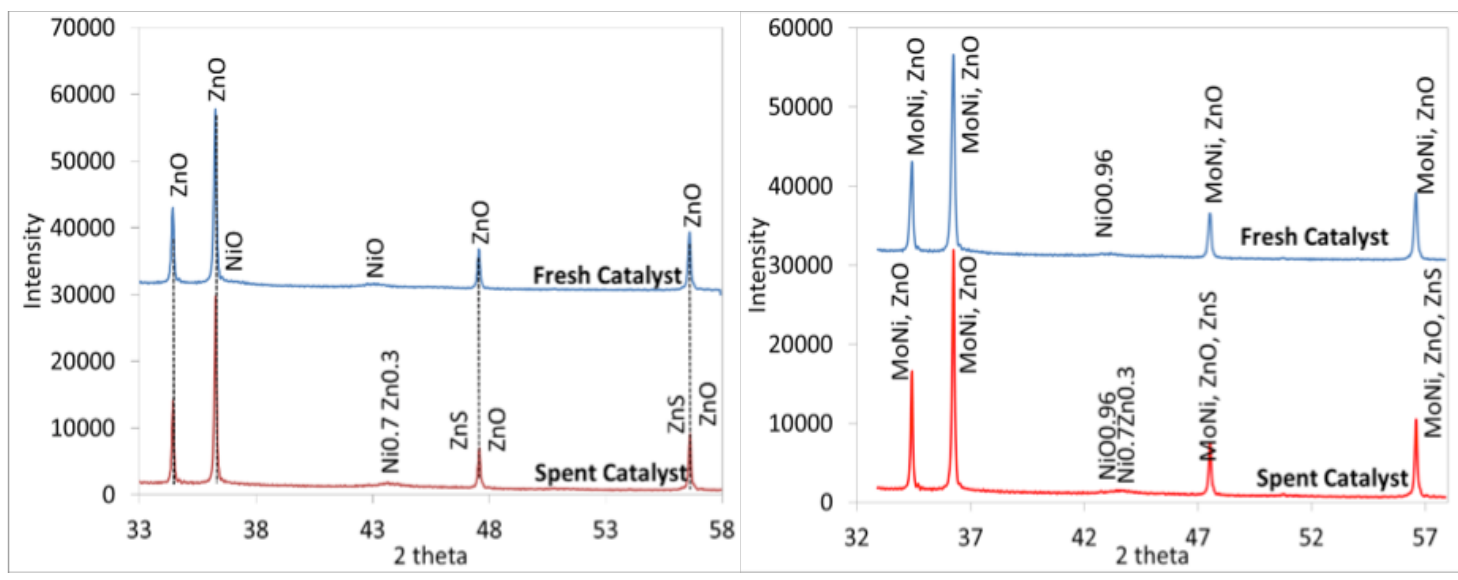

Figure 4.6.5: XRD patterns for fresh and spent catalysts with compositions (Left) 12\% $\mathrm{Ni}, 58.7 \% \mathrm{ZnO}, 29.3 \% \mathrm{y}-\mathrm{Al}_{2} \mathrm{O}_{3}$; and (Right) $12 \% \mathrm{Ni}, 3 \% \mathrm{Mo}, 58.7 \% \mathrm{ZnO}, 26.3 \% \mathrm{y}-$ $\mathrm{Al} 2 \mathrm{O} 3$

Figure 4.6.6 (a) and (b) show TEM micrographs of $\mathrm{ZnO}$ nanowire decorated with $\mathrm{NiO}$ nanoparticles in fresh catalyst and nickel-zinc nanoparticles in spent catalyst, respectively. The crystalline size (from XRD) for $\mathrm{NiO}$ nanoparticles in fresh catalyst was $10.4 \mathrm{~nm}$ and for spent catalyst nickel-zinc crystallite size was $12.8 \mathrm{~nm}$. This shows that the nanoparticles are not agglomerating/sintering to form bigger particles which usually happen with nanoparticles at these reaction conditions. One of the main problems with NPs is sintering [109]. For example, small nanoparticles of $\mathrm{ZnO}$ easily agglomerated which caused a catastrophic decrease in both desulfurization activities and sulfur capacities [109]. The formation of bigger particles would be detrimental for steady activity of the catalyst. This observation shows that nanowire supports have prevented the nanoparticles from sintering. This is one of the major qualities of these nanowire-based catalysts. NW morphology was retained, an important characteristic for their continued and steady activity. 


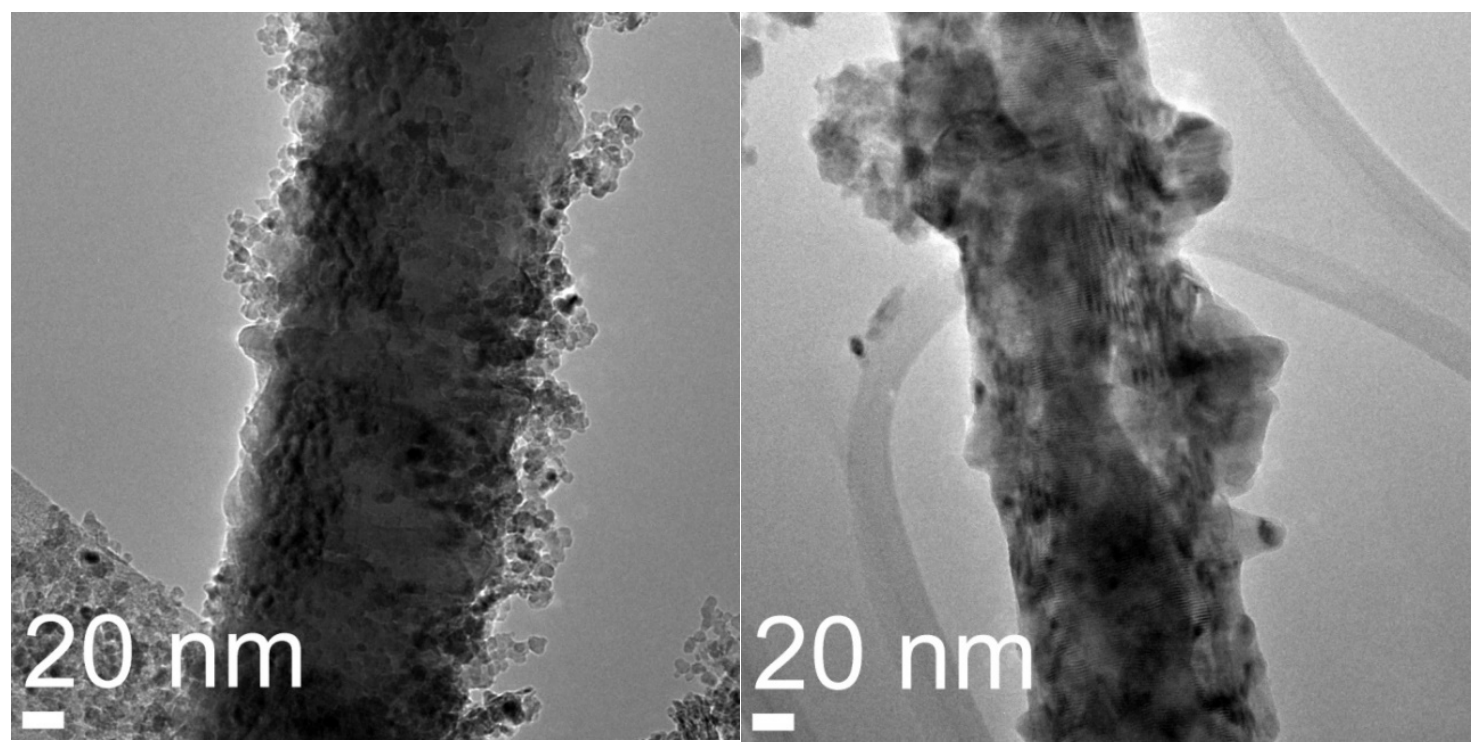

(a)

(b)

Figure 4.6.6: TEM micrographs of 15\% Ni, 58.7\% $\mathrm{ZnO}, 26.3 \% \gamma-\mathrm{Al} 2 \mathrm{O} 3$ (a) fresh catalyst; (b) spent catalyst. Images do not show the same nanowire but different ones from the same sample.

In order to further investigate the sulfur adsorption mechanism, a spent catalyst $\left(12 \% \mathrm{Ni}, 58.7 \% \mathrm{ZnO}, 29.3 \% \gamma-\mathrm{Al}_{2} \mathrm{O}_{3}\right)$ has been analyzed using high resolution TEM (HRTEM) micrographs and the selected-area electron diffraction (SAED) patterns of $\mathrm{Ni}$ and NiO. In Figure 4.6.7, the HRTEM images of the different regions of a spent ZnO NW decorated with nickel nanoparticle (A) show that: (B) the nickel cluster on the NW contains both metallic $\mathrm{Ni}$ and $\mathrm{NiO}$; (C) the $\mathrm{ZnS}$ was found on the $\mathrm{NW}$ with the lattice dspacings of $3.2 \AA$ and $3.17 \AA[110]$, corresponding to the (002) and the (100) planes, respectively, which are consistent with the ZnS phase (JCPDS Card No. 80-0007); (D) some unreacted $\mathrm{ZnO}$ presents in the core area of the NW with a fringe spacing of $5.27 \AA$, corresponding to the separation between the (0001) planes [111]. Therefore, this HRTEM 
study also strongly supports the findings from XRD that there is $\mathrm{ZnS}$ not NiS phase in spent catalysts.

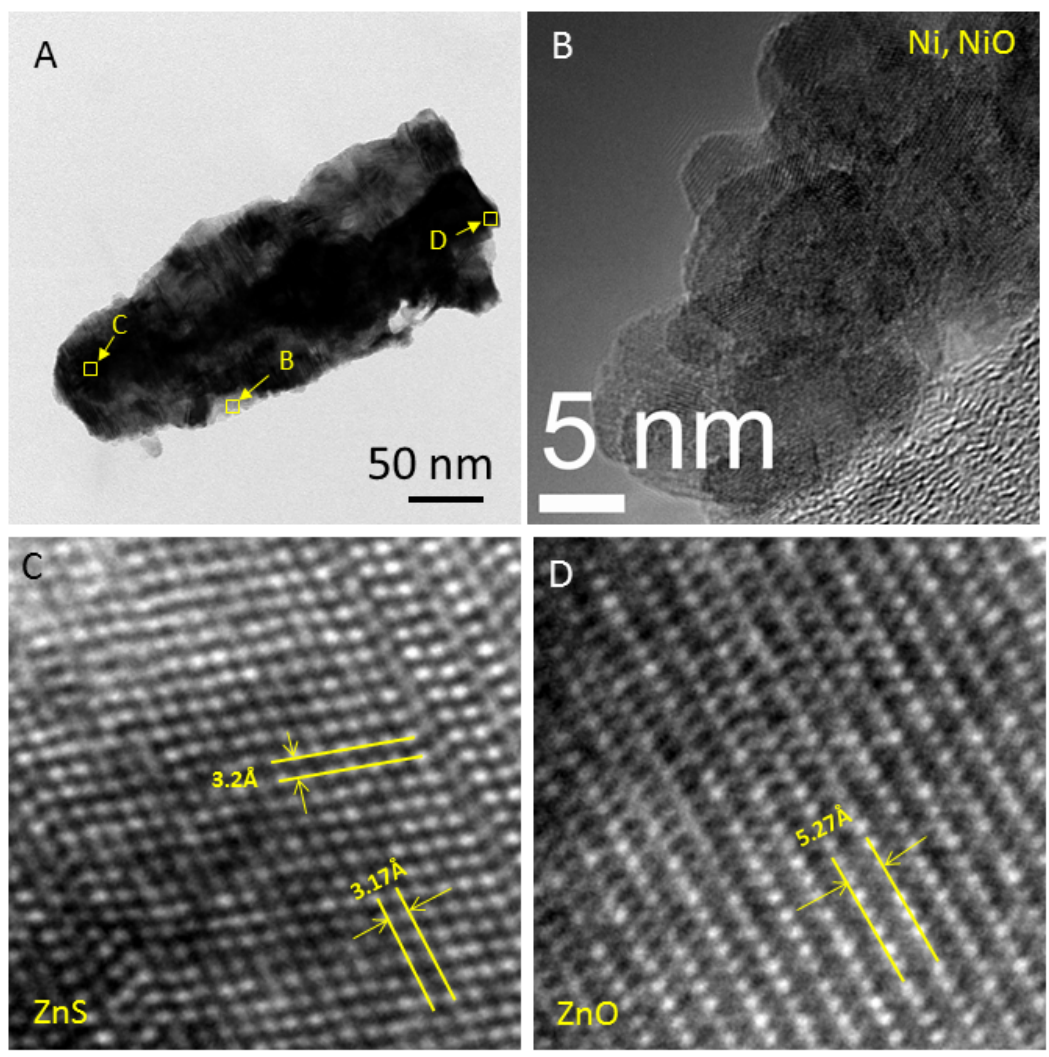

Figure 4.6.7: TEM micrographs of spent catalyst $\left(12 \% \mathrm{Ni}, 58.7 \% \mathrm{ZnO}, 29.3 \% \gamma-\mathrm{Al}_{2} \mathrm{O}_{3}\right)$ : (A) Low-magnification bright filed TEM micrograph; (B) HRTEM image of the selected area in catalyst (A): showing the $\mathrm{Ni}$ and $\mathrm{NiO}$ cluster; (C) HRTEM image of the selected area in catalyst (A): showing the tail area of the catalyst; (D) HRTEM image of the selected area in catalyst (A): showing the center area of $\mathrm{ZnO} \mathrm{NW}$.

Figure 4.6.8 shows the first hydrogenation of 4,6-DMDBT. This path is more probable than direct desulfurization (DDS) because sterically hindered C-S bond is easier to break when one of the aromatic rings is first hydrogenated. This step makes sulfur atom more accessible to the active site. Then sulfur is then removed via hydrogenolysis 
of the C-S bond on the $\mathrm{Ni}_{\mathrm{x}} \mathrm{Zn}_{\mathrm{y}}$ site. This accelerated pre-hydrogenation activity is critical in removing highly refractive sulfur compounds such as 4,6-DMDBT [96, 112]. Fresh metal sites provide the suitable environment for the hydrogenation to occur first. The sulfur can directly transfer (solid state diffusion) from $\mathrm{Ni}_{\mathrm{x}} \mathrm{SZn}_{\mathrm{y}}$ to $\mathrm{ZnO}$. In this mechanism, the NW morphology provides for easier sulfurization and sulfur transfer from active metal particles.
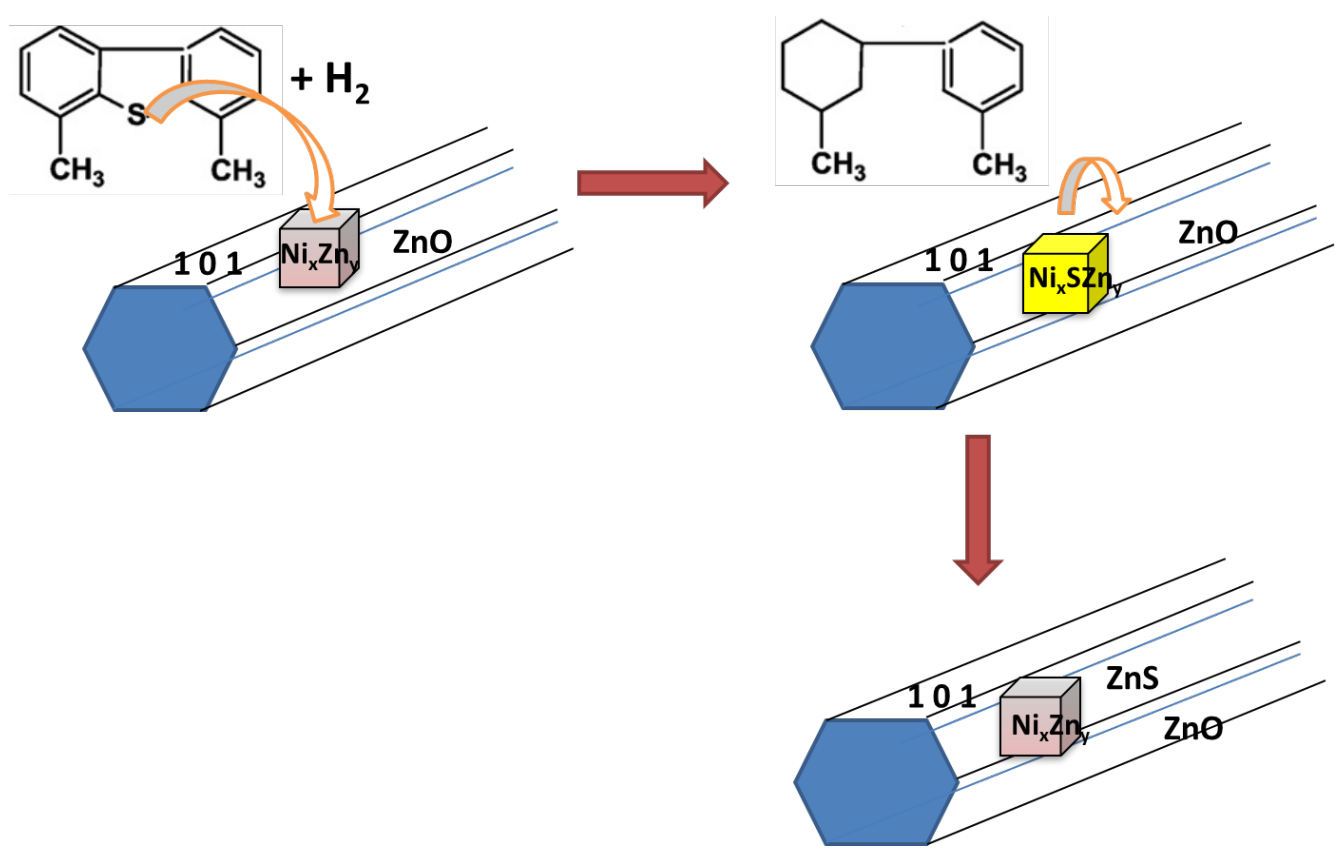

Figure 4.6.8: Proposed reaction mechanism for desulfurization on $\mathrm{Ni} / \mathrm{ZnO}$ nanowires

\subsubsection{Conclusions}

Different catalysts containing 12-30\% Ni clusters on $\mathrm{ZnO}$ nanowire supports and $\gamma-\mathrm{Al}_{2} \mathrm{O}_{3}$ were synthesized, characterized, and tested. The sulfur content in diesel was brought down to less than $1 \mathrm{ppm}$ from 30-200 ppm (approx.) sulfur in different feeds at moderate reaction conditions (pressure 30 bars and temperature $290^{\circ} \mathrm{C}$ ) compared to 
industrial practice $\left(60-90\right.$ bars and $\left.350-390^{\circ} \mathrm{C}\right)$. The data showed that our catalysts have shown to be highly active towards 4,6-Dimethyldibenzothiophene (DMDBT). The steady state activity of the catalyst was achieved within the first few hours and remained constant for over 100 hours (after which, the tests were discontinued). The active catalytic site was shown to be comprised of super-Ni-rich $\mathrm{Ni}_{\mathrm{x}} \mathrm{Zn}_{\mathrm{y}}$ phase, which remained essentially sulfur free after desulfurization process.

\subsection{Summary}

A flame based method for producing metal oxide such as zinc oxide nanowires at large scale is described using a new fluidized bed reactor. The reactor has been shown to produce nanowire using both microwave plasma and hydrocarbon. The nanowire produced using microwave plasma are about $20-80 \mathrm{~nm}$ in diameter, about 1 micron in length, aspect ratio of $6-20$. The nanowire produced using hydrocarbon are larger in diameter of about $80-150 \mathrm{~nm}$, about the same length of 1 micron, aspect ratio of $2-10$. The production rate as high as 2 kilograms per hour is obtained using hydrocarbon flame. The yield is about $30 \%$ using microwave plasma flame and about $90 \%$ using hydrocarbon. The nanowire morphology can be controlled by varying the operating parameters such as flame source, gas flow rate, feeding rate. The yield can be increased by increasing hydrocarbon flow rate and flame volume. The production capacity can be increased by increasing the feeding rate, hydrocarbon flow rate and flame volume. The fluidization is the key factor for archiving high yield and high production capacity.

The resulting zinc oxide nanowires showed excellent performance as catalyst support for hydro-desulfurization of diesel. The $12-30 \% \mathrm{Ni}$ clusters on $\mathrm{ZnO}$ nanowire supports and $\gamma-\mathrm{Al}_{2} \mathrm{O}_{3}$ were shown to remove sulfur content in diesel from $30-200 \mathrm{ppm}$ to 
less than $1 \mathrm{ppm}$. The steady state activity of the catalyst was achieved within the first few hours and remained constant for over 100 hours. 


\section{CHAPTER V}

\section{SCALABLE SOLVO-PLASMA PRODUCTION OF TIN OXIDE NANOWIRES}

\subsection{Scalable solvo plasma production of tin oxide nanowires}

\subsubsection{Introduction}

Tin oxide nanowires is one of the most promising 1D nanostructures for advanced applications in lithium-ion batteries[6], solar cells[50], sensors[113], optical

devices[114], and electronic devices[5]. However, the wide-spread use of nanowire based materials in to energy device applications has been limited due to the fact that there are no scalable manufacturing methods, yet. For example, the lithium-ion battery electrodes will require production on the order of kilograms even for simple pouch cell prototyping. Thus, the development of reliable, reproducible and simple techniques for production of tin oxide nanowires and their testing into energy devices is of great interest. Up to date, several approaches have been proposed to prepare tin oxide nanowires including chemical vapor deposition[115], laser ablation[116], template method[117] and most predominantly hydrothermal technique[118]. 
Hydrothermal synthesis has been traditionally used to prepare nanostructured materials including nanoparticles and nanowires. However, this approach requires long time scale, high pressure, multiple steps and expensive precursors [119]. Further, in most cases, hydrothermal techniques require the use of surfactant to control the nanowire growth. Also, no clear mechanism for tin oxide nanowires have been proposed. Importantly, the hydrothermal techniques are limited to lab scale (few milligrams to hundred milligrams a batch) $[120,121]$. Two recent hydrothermal based approaches are microwave assisted hydrothermal and continuous flow hydrothermal method, which can increase the production rate to about $5 \mathrm{~g} /$ day and $10 \mathrm{~g} /$ hour, respectively $[122,123]$. However, these hydrothermal methods have been shown to work only for nanoparticles (NPs) and not to make nanowires. Another popular method for making nanowire growth is that using vapor-liquid-solid (VLS) process for many semiconductors including tin oxide [115]. However, VLS processes require the use of low pressure, high temperatures, high fabrication cost and $\mathrm{Au}$ as catalyst.

In this chapter, we report a facile, fast "solvo-plasma" production of tin oxide nanowires using tin oxide particles as a low-cost source. This study demonstrates, for the first time, the direct conversion of tin oxide particles to tin oxide nanowires with reaction time scales on the order of a minute or lower. More importantly, various experimental studies are used to understand the underlying principle of nucleation and growth of tin oxide nanowires in the presence of alkali hydroxides. 


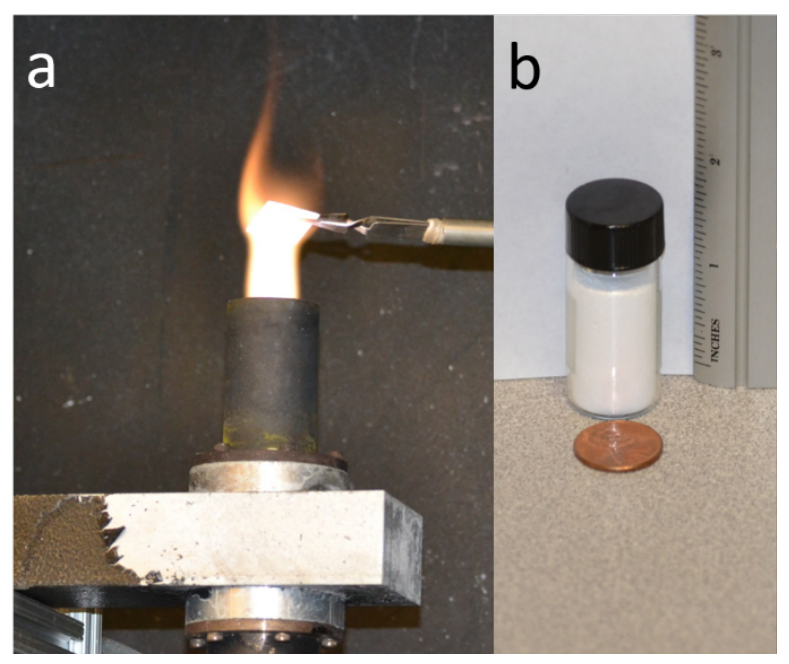

Figure 5.1.1: Photograph of an actual experiment: plasma exposure of tin oxide precursor and $\mathrm{KOH}$ paste on quartz substrate a), a bottle of tin oxide nanowire powder of 10 grams produced in one hour reaction time

\subsubsection{Results and Discussion}

All experiments are performed using inexpensive, commercial tin oxide particles without any further purification by mixing them with $\mathrm{KOH}$ and exposing to atmospheric plasma flame for 15 seconds to 1 minute. Using a 1 -inch square substrate, we were able to demonstrate production of tin oxide nanowires at a scale of 10 grams in one hour (Figure 5.1.1b). The exposure time used here is less than a minute which is about 3.5 orders of magnitude faster than that using a hydrothermal method. Figure 5.1.2a shows SEM images of the resulting tin oxide nanowires that are 2 microns in length and about 50 to $100 \mathrm{~nm}$ in diameter. The TEM images of tin oxide nanowires show highly porous structures of tin oxides, which is due to $\mathrm{HCl}$ ion exchange and plasma annealing step (Figure 5.1.2b, c). The BET surface area of the porous tin oxide nanowires turned out to be $37 \mathrm{~m}^{2} / \mathrm{g}$. The BET surface area could be increased further by controlling the ion 
exchange and annealing parameters. The phase transformation of intermediate nanowires to tin oxide nanowires created the porous structures where potassium and water vapor are removed. The porous structures consisted of interconnected tin oxide nanoparticles. The underlying mechanism for porous structure formation seems to be similar to that suggested in many other reports using hydrothermal synthesis with additional annealing step[118]. However, the actual mechanism is not completely clear in terms of how phase transformation leads to porous nature within the nanowires. Figure 5.1.2c also revealed the twining effect where the boundary in the top left corner clearly showed merging of two nanowires. The merging maybe due to the high surface diffusion of tin atoms on nanowire surface under plasma exposure [60]. The HR-TEM image in Figure 5.1.2d showed the match of interplanar lattice spacing of $3.35 \AA$ and $2.64 \AA$ for the plane (110) and (101), respectively. 


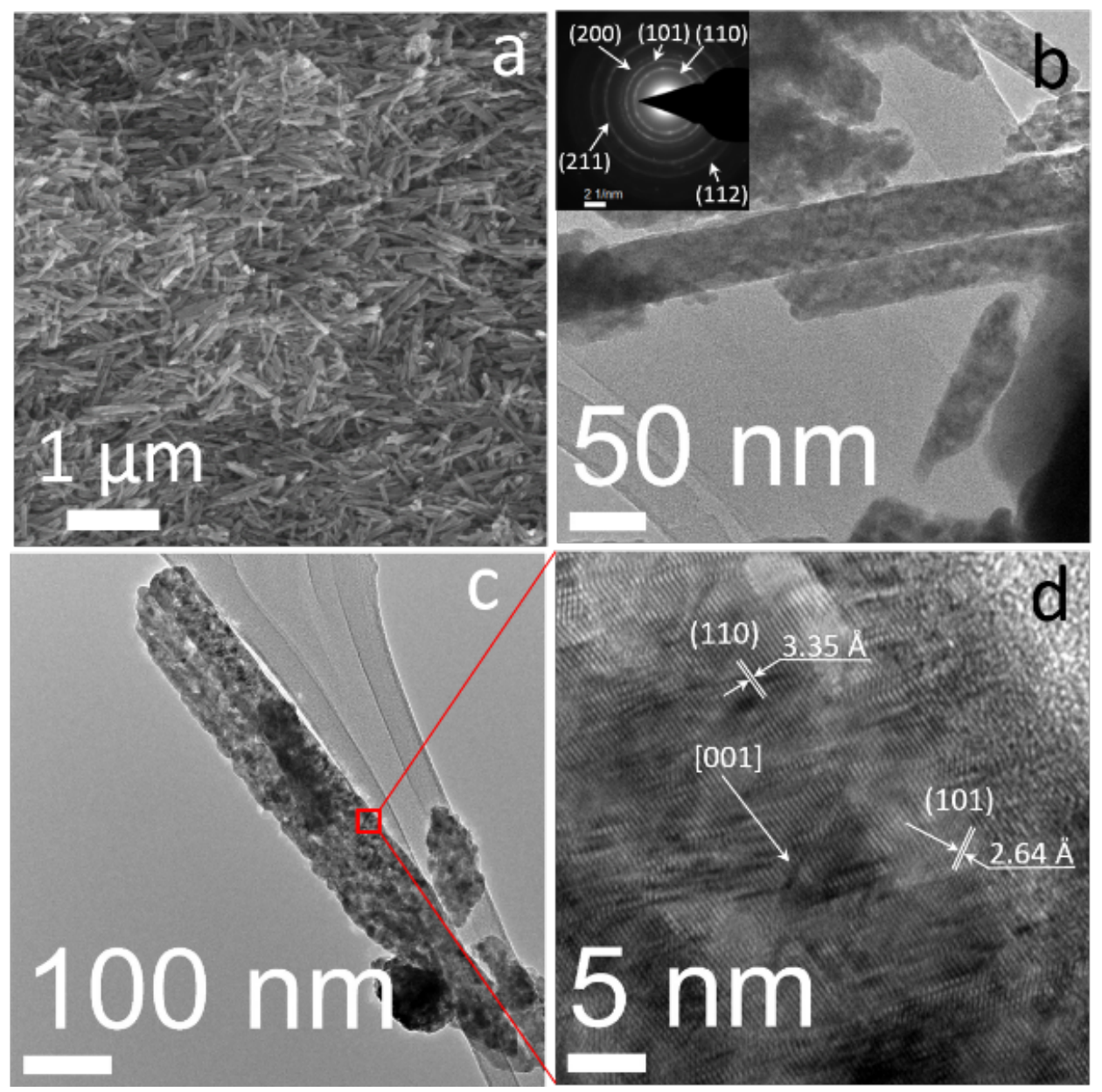

Figure 5.1.2: Scanning electron microscopy of tin oxide nanowire powder after annealing a), TEM images b) and c) showing the porous structure and twining effect, (inset) SAED of tin oxide nanowires, HR-TEM image showing the matching interplanar lattice spacing of (110) and (101) plane of tin oxide nanowires d). 


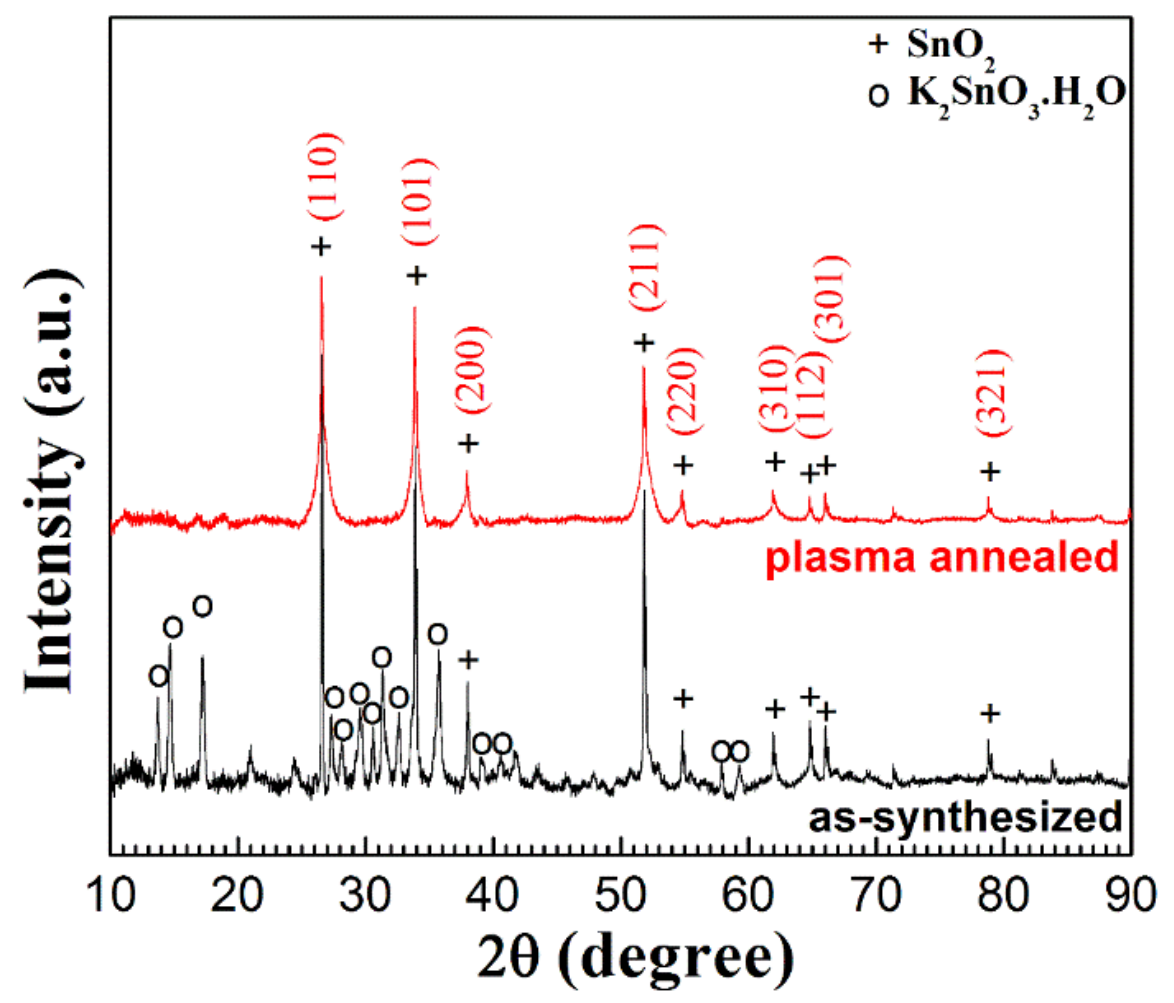

Figure 5.1.3: Powder XRD pattern of samples after annealing in plasma showing the pure phase of rutile tin oxide while the as-synthesized samples showing additional intermediate compounds. 


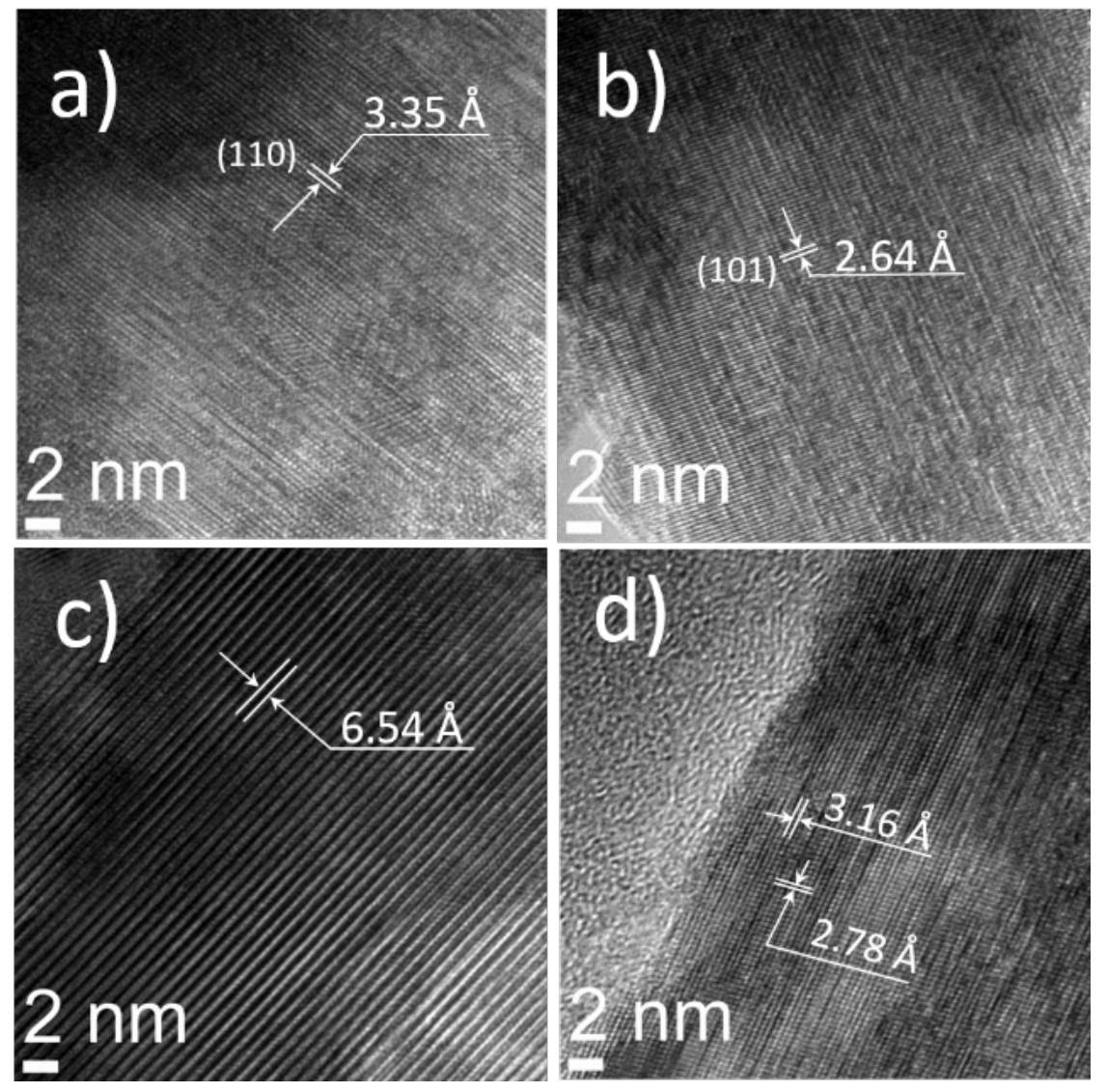

Figure 5.1.4: HR-TEM images of as-synthesized samples showing well-matched interplanar lattice spacing of rutile tin oxide in (a) and (b), $\mathrm{K}_{2} \mathrm{SnO}_{3} \cdot \mathrm{H}_{2} \mathrm{O}$ in (c) and (d) 


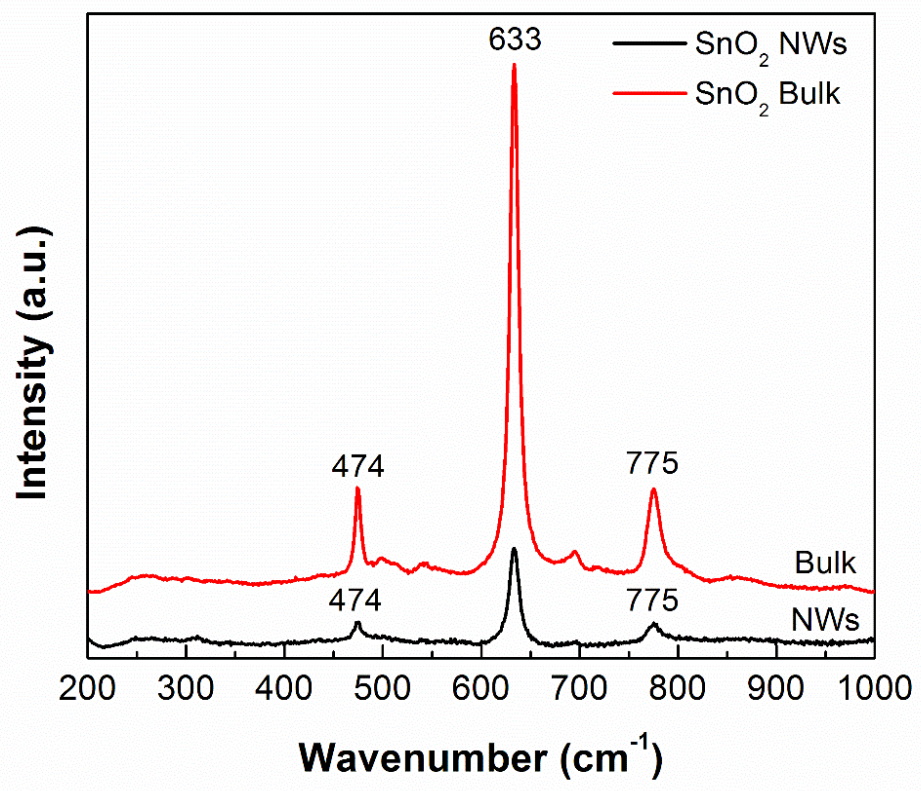

Figure 5.1.5: Raman spectrum of tin oxide after plasma annealing in comparison with bulk tin oxide

The powder XRD pattern of tin oxide nanowires after annealing in plasma is shown in Figure 5.1.3. The diffraction peaks revealed that tin oxide nanowires have tetragonal crystal structure with lattice parameters $\mathrm{a}=\mathrm{b}=4.74 \AA$ and $\mathrm{c}=3.18 \AA$, corresponding bulk rutile tin oxide (PDF-00-005-0467). The selected area diffraction pattern also confirms the pure phase tin oxide as in Figure 5.1.2b. No other impurity peaks are detected in the tin oxide nanowire samples after annealing in plasma. The Raman spectroscopy of the tin oxide nanowires after annealing shows fundamental peaks at 474,633 , and $775 \mathrm{~cm}^{-1}$ that is well matched with rutile tin oxide[124] (Figure 5.1.5). In addition to the peaks of tin oxide phase, the XRD pattern of as-synthesized samples (Figure 5.1.3) showed peaks of $\mathrm{K}_{2} \mathrm{SnO}_{3} \cdot \mathrm{H}_{2} \mathrm{O}$ (PDF-00-019-0993). The HR-TEM images showed well-matched interplanar lattice spacing of $\mathrm{SnO}_{2}$ (Figure 5.1.4a, b) and layered structure $\mathrm{K}_{2} \mathrm{SnO}_{3} \cdot \mathrm{H}_{2} \mathrm{O}$ (Figure 4c, d). The lattice spacing of $6.54 \AA, 3.16 \AA$ and $2.78 \AA$ 
correspond to the peaks of $13.5^{\circ}, 28.2^{\circ}$, and $32.2^{\circ}$ of $\mathrm{K}_{2} \mathrm{SnO}_{3} \cdot \mathrm{H}_{2} \mathrm{O}$. EDS analysis shows that the as-synthesized nanowires contain about 13 at. $\%$ of potassium. This confirms that the intermediate compound or as-synthesized compound is $\mathrm{K}_{2} \mathrm{SnO}_{3}$. The hkl index of $\mathrm{K}_{2} \mathrm{SnO}_{3} \cdot \mathrm{H}_{2} \mathrm{O}$ is not available to our knowledge. However, the lattice spacing of tin oxide in Figure 5.1.2d shows that tin oxide nanowires grown along [001] direction that is in agreement with previous reports $[119,125]$. [001] direction has highest surface energy that favored the 1D growth $[120,126]$.
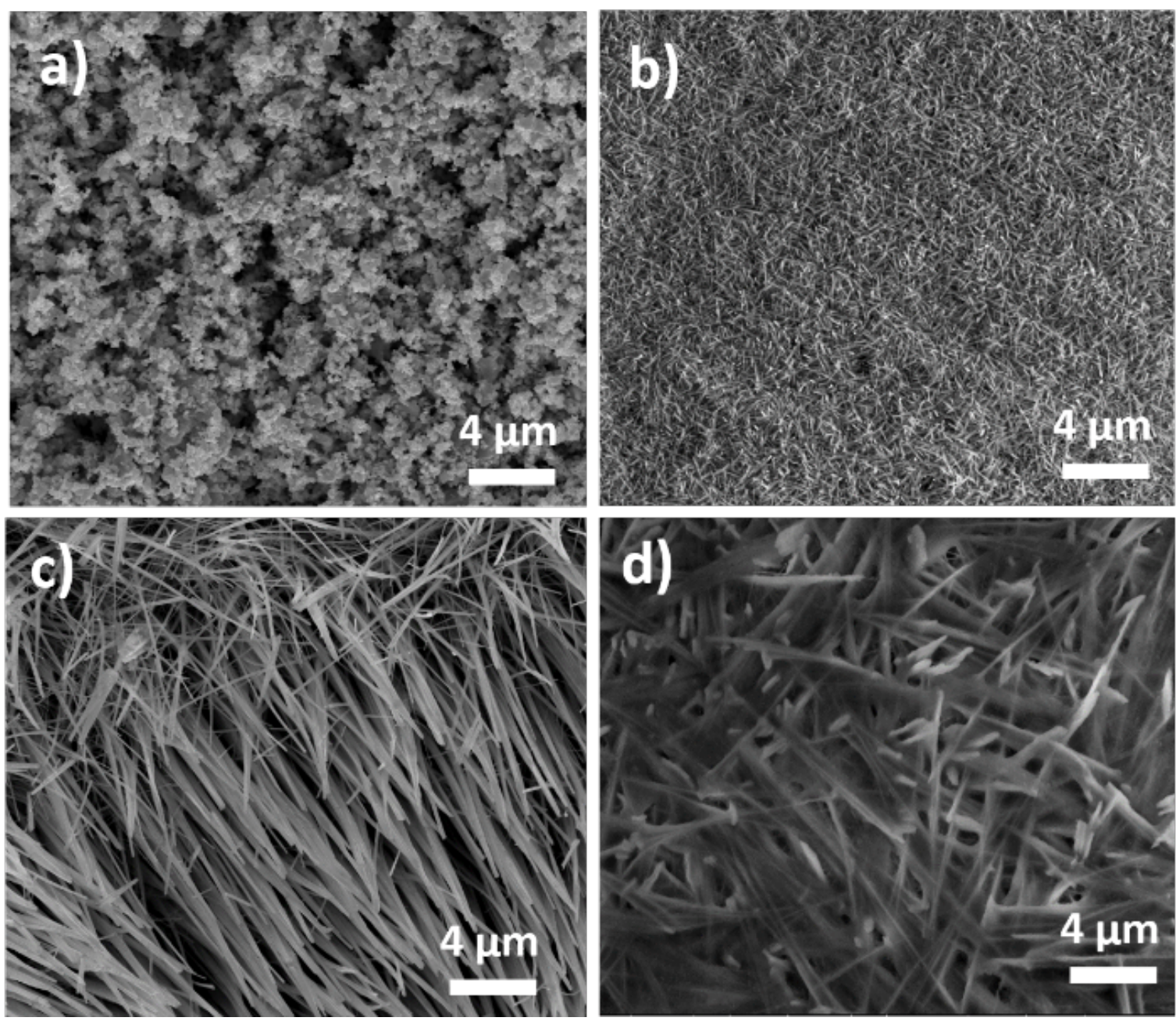
Figure 5.1.6: SEM images of (a) tin oxide precursor and as-synthesized $\mathrm{K}_{2} \mathrm{SnO}_{3}$ nanowires at weight ratios of $\mathrm{SnO}_{2}$ to $\mathrm{KOH}$ of (b) 3:1, (c) 1:1, (d) 1:3. Experiments were conducting using plasma flame with power of $1.2 \mathrm{~kW}$ at a duration of 60 seconds

Even though, the above results clearly demonstrate the formation of onedimensional structures with plasma exposure of tin oxide powder mixed with $\mathrm{KOH}$, there are several questions remain unanswered about the observed 1-D growth. So, experiments were conducted for different weight ratios of $\mathrm{SnO}_{2}$ to $\mathrm{KOH}$ to understand initial stages of growth. Figure 5.1.6 showed the SEM images of as-synthesized samples at different weight ratios of $\mathrm{SnO}_{2}$ to $\mathrm{KOH}$ in comparison with the tin oxide particle precursor. Thin, short nanowires of about $70 \mathrm{~nm}$ in diameter and 2 to 4 microns in length are observed at weight ratio of 3 to 1 . When the weight ratio for precursors decreased to 1 to 1 , there is more change in the nanowire diameter and length. As shown in Figure 5.1.6c, the resulting nanowires are of 100 to $300 \mathrm{~nm}$ in diameter and 20 to 30 microns in length. The image also shows that nanowires bunch up and seem to be fusing together for samples grown using precursors at a weight ratio of 1 to 1 . Such sintering of nanowires could be the reason for twining effect seen in Figure 2c. The nanowire diameter increased further when using precursor ratio to 1:3. However, there is the presence of excess $\mathrm{KOH}$ on top of nanowires that make it difficult to quantify diameters. The increase in diameter for resulting nanowires can probably be explained with decreasing supersaturation with decreasing $\mathrm{SnO}_{2}$ to $\mathrm{KOH}$ ratio from 3:1 to 1:3. 

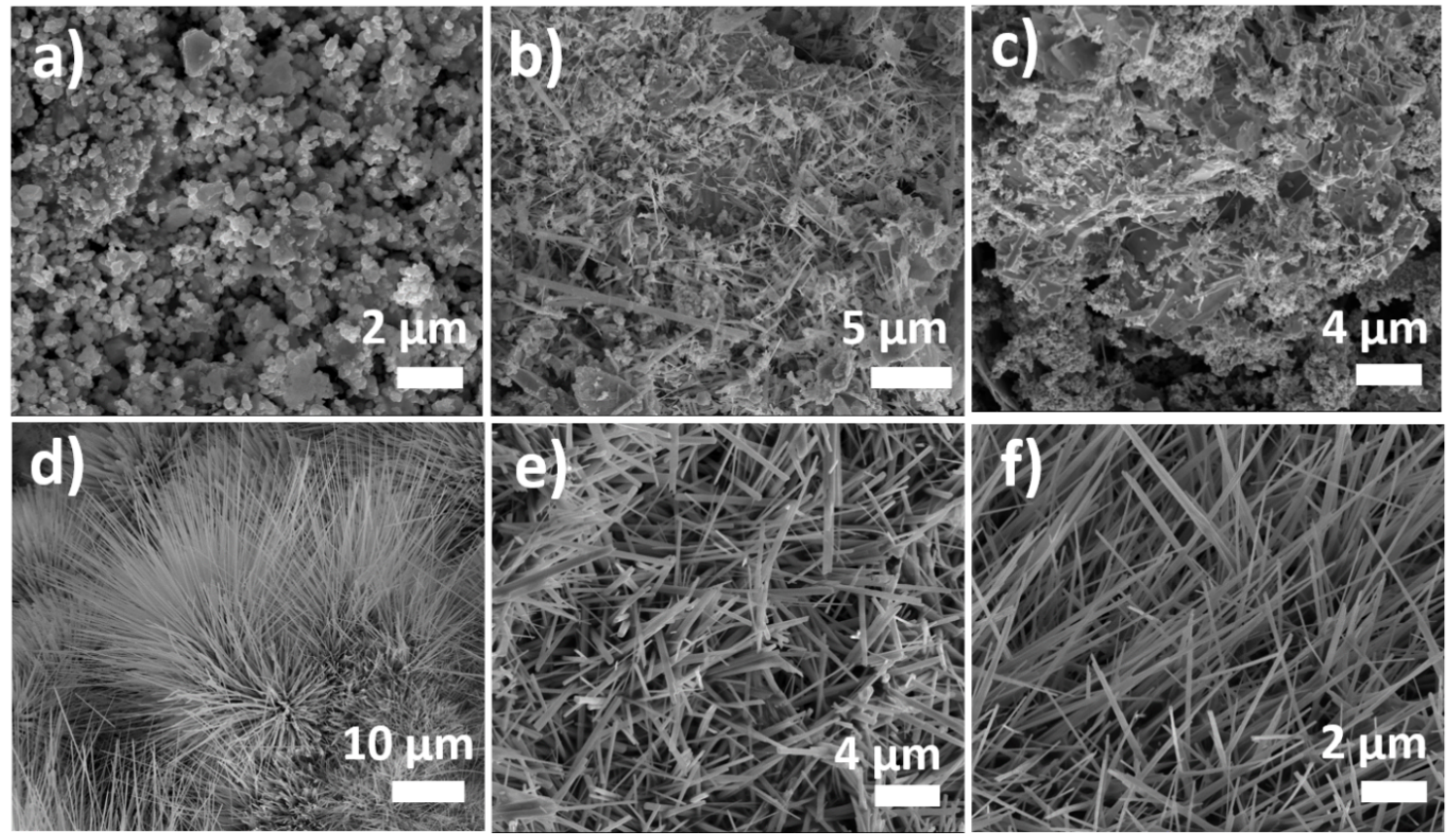

Figure 5.1.7: SEM image of as-synthesized samples using a) $\mathrm{SnO}_{2}+\mathrm{KCl}$, b) $\mathrm{Sn}+\mathrm{KOH}$, c) $\mathrm{Sn}+\mathrm{KCl}$, d) $\mathrm{SnO}+\mathrm{KOH}$, e) $\mathrm{K}_{2} \mathrm{SnO}_{3}$, f) $\mathrm{SnO}_{2}+\mathrm{KOH}$. Sample a) to e) prepared using plasma heating for 60 seconds, sample f) prepared using thermal heating at $900^{\circ} \mathrm{C}$ for 2 hrs.

Experiments were also performed using different tin precursors such as tin oxide, tin and tin monoxide to determine their impact on reaction time scales and nanowire growth rates. As shown in Figure 5.1.7, $\mathrm{K}_{2} \mathrm{SnO}_{3}$ nanowires are obtained using a mixture of tin metal powder or tin mono-oxide particles with $\mathrm{KOH}$. Nanowires obtained using tin metal powder have random diameters and lengths. Nanowires obtained using tin monooxide have diameters of about 50 to $100 \mathrm{~nm}$ and lengths up to 50 microns (Figure 5.1.7d). In fact, tin oxide nanoparticles are always found together with $\mathrm{K}_{2} \mathrm{SnO}_{3}$ nanowires when tin metal powder used as starting material (Figure 5.1.7b, c). No nanowires formed with either mixture of $\mathrm{H}_{2} \mathrm{O}$ or $\mathrm{KCl}$ with tin oxide particles (Figure 5.1.7a). In another 
experiment, the same mixture of tin oxide particles and $\mathrm{KOH}$ at mass ratio of 3 to 1 is put onto a quartz substrate and was thermally heated to $900^{\circ} \mathrm{C}$ for 2 hours in a box furnace. The similar tin oxide nanowire morphology but longer in length is observed (Figure 5.1.7f).
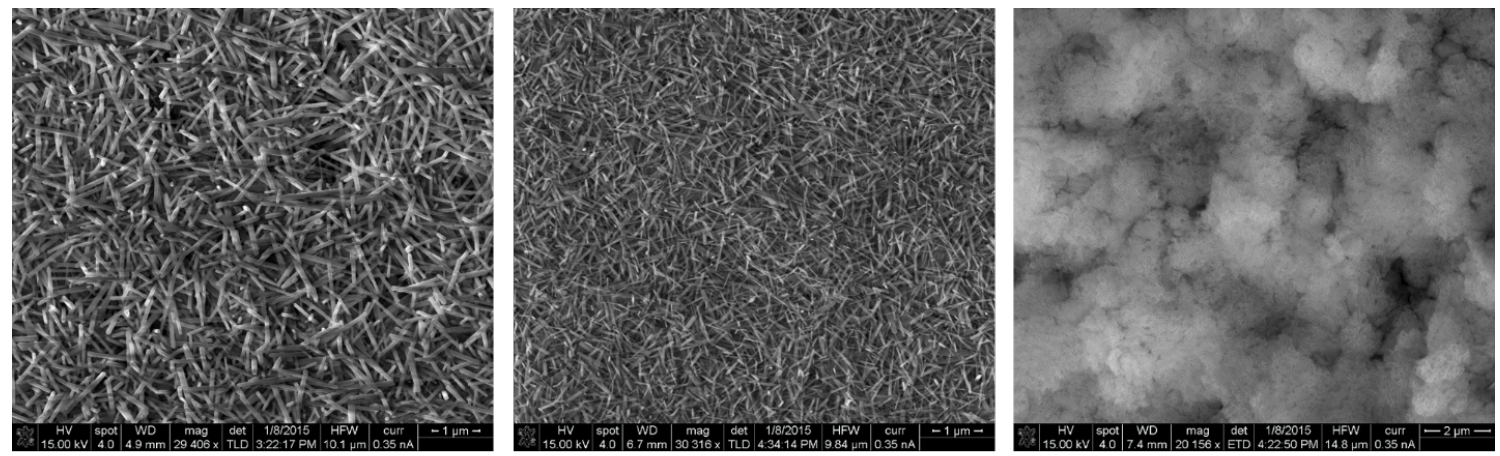

a)

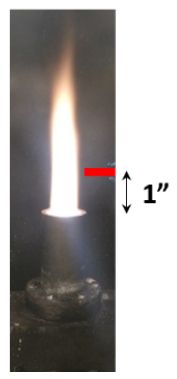

b)
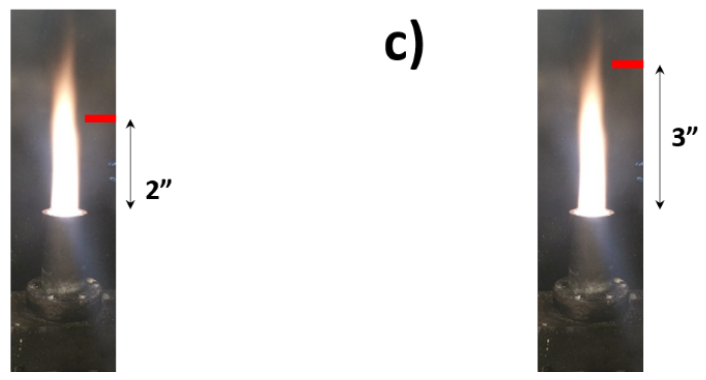

Figure 5.1.8: SEM image of as-synthesized sample prepared at different distance away from the outlet of applicator: a) 1 inch, b) two inches, and c) 3 inches. The bottom row showing the actual plasma flame with red marks as position of samples

Plasma is a partially ionized gas which include highly active species such as electrons, ion, neutrals. Plasma density, which is related directly to density of such active species, has been shown to have a strong effect on nanostructure formation. Our facility has no access to measure plasma density. However, plasma density gradient along the plasma flame can be used to study the effect of plasma density on formation of our nanowires. The highest plasma density is at the outlet of application. The plasma density decreases as we move far away from the outlet of applicator as shown in bottom row in 
Figure 5.1.8. As observed in Figure 5.1.8, high density, uniform nanowire diameter of about $70 \mathrm{~nm}$ and 2-5 microns in length was obtained when precursor paste was exposed to plasma flame at a distance of 1 inch. At the distance of 2 inches, the nanowire became shorter in length and thinner in diameter. Eventually, there was no nanowire formation at the distance of 3 inches. At 3 inches away, the plasma density was low or oxygen radical concentration was low which resulted in low concentration of $\mathrm{K}_{2} \mathrm{SnO}_{3}$. Thus, supersaturation of $\mathrm{K}_{2} \mathrm{SnO}_{3}$ was not achieved for growth of nanowires.
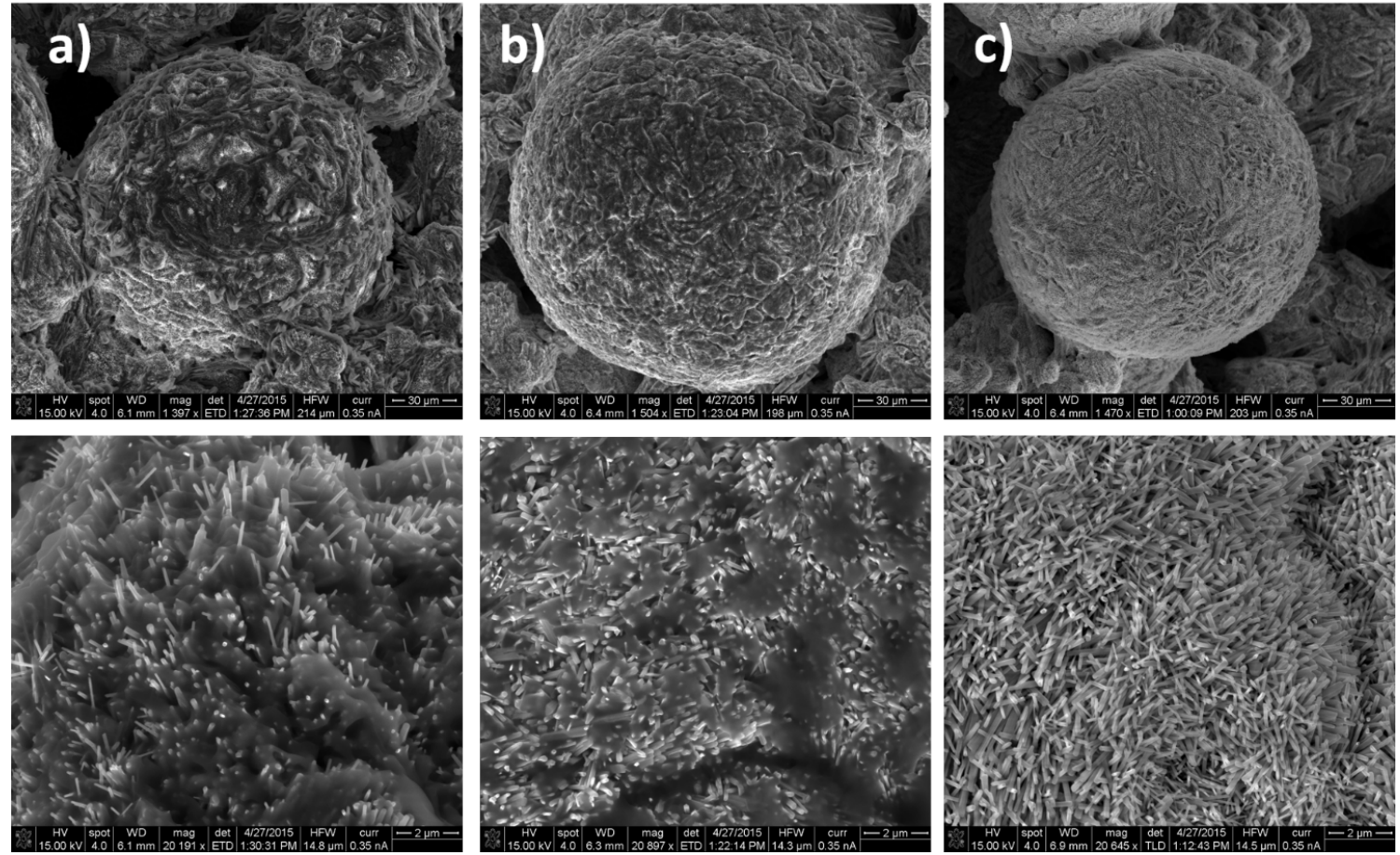

Figure 5.1.9: SEM image of as-synthesized sample prepared at different time scale using large tin oxide particles as precursor: a) 20 seconds, b) 60 seconds, and c) 5 minutes. Lower row showing images at higher magnification

Micron size of tin oxide particles has been converted to tin oxide nanowires completely in the early results. Experiments also were conducted using larger tin oxide particles as large as 150 microns in diameter. As shown in Figure 5.1.9, the stannate 
nanowires were prepared using large tin oxide particles. Unlikely the case of 1-5 microns' tin oxide particle used where the tin oxide particles were completed melted or collapsed into thin film on the substrate, the large tin oxide particle morphology was still maintained. However, there were stannate nanowires all over the surface of tin oxide precursor particles. As the reaction time increased from 20 seconds to 5 minutes, the nanowires became denser. However, the nanowire length remained as same as those of 15 microns tin oxide precursor particles. This can be explained by the diffusion of oxygen radical into large particle size. Due to large particle size of tin oxide precursor particles, the oxygen radical could not reach the core of the tin oxide particle and thus the tin oxide particles remained the same spherical morphology. There was only thin layer on the surface of tin oxide particle got reacted. As a result, the short nanowires as same as those of 1-5 microns tin oxide particles.

\subsection{Growth mechanism for stannate nanowires during solvo-plasma oxidation}

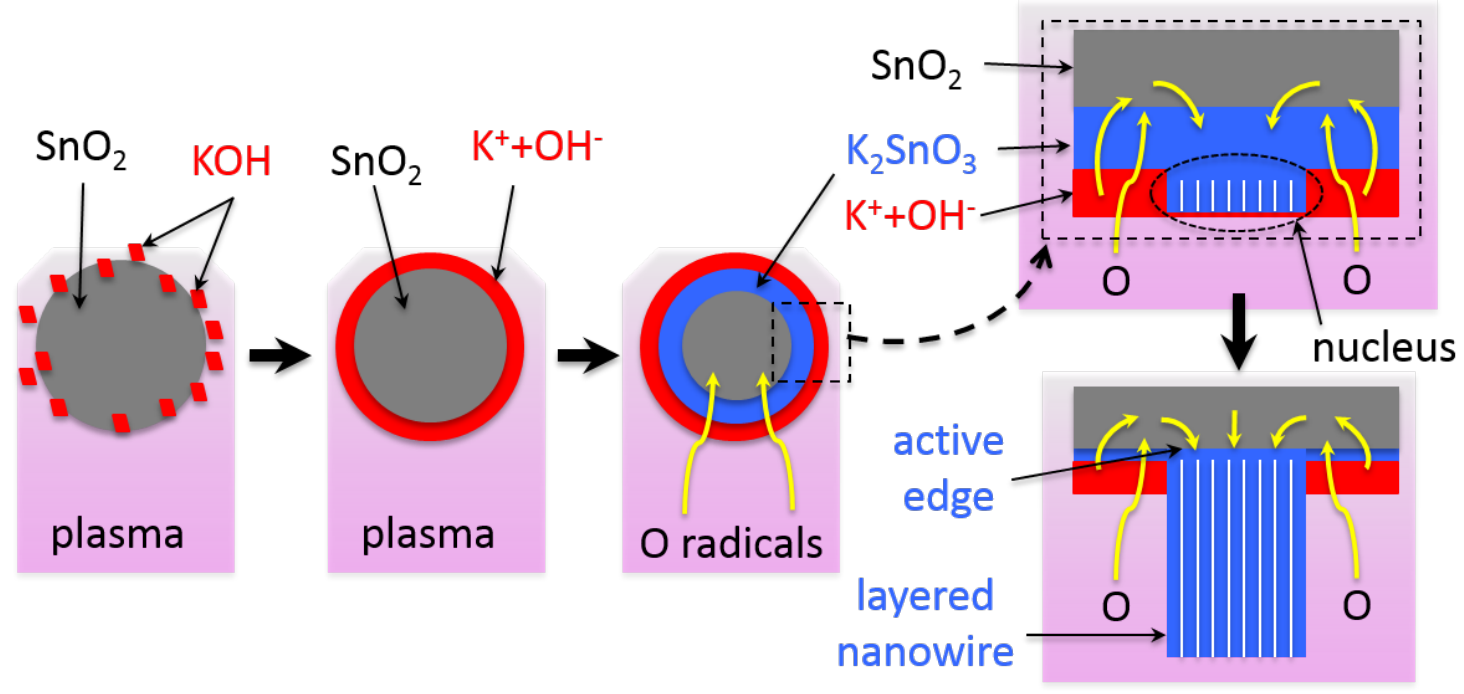

Figure 5.2.1: Schematic showing the proposed growth mechanism for growth of tin oxide nanowires. a) Mixture of tin oxide particle and $\mathrm{KOH}$ in plasma flame, b) $\mathrm{KOH}$ got melted 
into free moving $\mathrm{K}^{+}$and $\mathrm{OH}^{-}$ions, c) diffusion of oxygen radicals, $\mathrm{K}^{+}$and $\mathrm{OH}^{-}$ions into tin oxide particle to form $\mathrm{K}_{2} \mathrm{SnO}_{3}$, d) $\mathrm{K}_{2} \mathrm{SnO}_{3}$ nucleus forming under supersaturation of $\mathrm{K}_{2} \mathrm{SnO}_{3}$, e) layered structure $\mathrm{K}_{2} \mathrm{SnO}_{3}$ grown due to active edge.

Based on all the experimental observations, we propose a mechanism to explain tin oxide nanowire growth in a three step sequence as shown in Figure 5.2.1: (1) continuous dissolution of tin oxide particles with $\mathrm{KOH}$ results in supersaturation of tin oxide in molten phase containing K-Sn-O, (2) phase segregation and bulk nucleation of $\mathrm{K}_{2} \mathrm{SnO}_{3}$ on the surface of tin oxide particles due to non-stable molten phase containing $\mathrm{K}$ Sn-O, (3) $\mathrm{K}_{2} \mathrm{SnO}_{3}$ formation started from surface of tin oxide particles by diffusiondriven self-arrangement and basal attachment[127]. The resulting $\mathrm{K}_{2} \mathrm{SnO}_{3} \cdot \mathrm{H}_{2} \mathrm{O}$ phase has layered structure with active sites at the edge sites for faster attachment kinetics. Faster growth kinetics along certain planes will only lead to platelet structure and morphology. So, in addition to faster growth kinetics at edges of layered structure, selective wetting of crystal nuclei with molten phase is hypothesized for ensuring one-dimensional growth for $\mathrm{K}_{2} \mathrm{SnO}_{3}$ nanowires. Extra exposure of $\mathrm{K}_{2} \mathrm{SnO}_{3}$ nanowires also result in tin oxide nanowires. So, the final product can contain pure tin oxide nanowires. But, based on our experimental data, the formation of $\mathrm{K}_{2} \mathrm{SnO}_{3}$ 1-D structures occurs first and then transforms to pure phase tin oxide nanowires. Previous studies using hydrothermal method suggested that the formation of tin oxide nanowires was due to decomposition of intermediate phase of $\mathrm{Na}_{2} \mathrm{Sn}(\mathrm{OH})_{6}$ to tin oxide nuclei followed by condensation to form tin oxide nanowires $[119,128]$. 


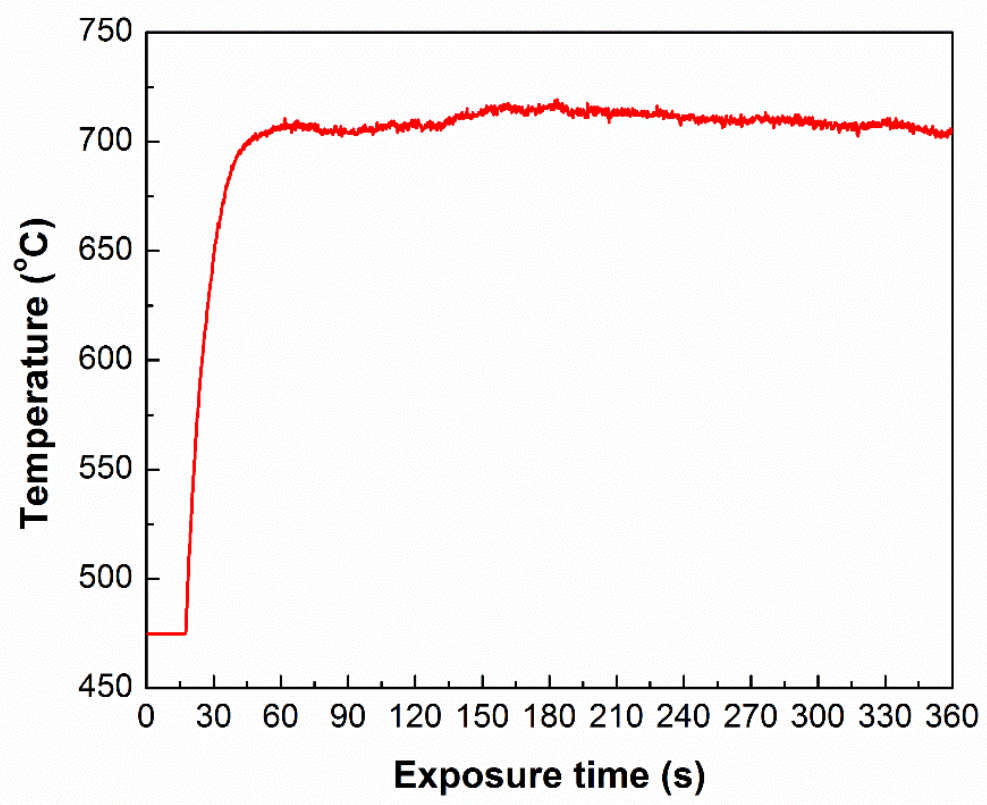

Figure 5.2.2: In-situ measurement of surface temperature of samples on quartz substrate

In principle, $\mathrm{KOH}$ can easily be melted into free moving $\mathrm{K}^{+}$and $\mathrm{OH}^{-}$ions under plasma exposure or thermal heating up to $406^{\circ} \mathrm{C}$ or higher. Indeed, an in-situ measurement of surface temperature using a Pyrometer which showed the temperature to be lower than its minimum detection limit of $475^{\circ} \mathrm{C}$ after 17.4 seconds of exposure time. $\mathrm{KOH}$ probably melted sooner than 10 seconds of exposure time as shown in Figure 5.2.2. Free moving $\mathrm{K}^{+}$and $\mathrm{OH}^{-}$ions and oxygen radicals[129, 130] produced by atmospheric plasma flame quickly diffuse into tin oxide structure to form a thin layer of molten melted of $\mathrm{K}_{2} \mathrm{SnO}_{3}$ on the surface of tin oxide particles.

$$
\mathrm{SnO}_{2}+2 \mathrm{~K}^{+}+2 \mathrm{OH}^{-}+\mathrm{O} \rightarrow \mathrm{K}_{2} \mathrm{SnO}_{3}+\mathrm{H}_{2} \mathrm{O}+1 / 2 \mathrm{O}_{2}
$$

Fast diffusion of $\mathrm{K}^{+}, \mathrm{OH}^{-}$ions and oxygen radicals into tin oxide particles make tin oxide quickly dissolved to form a molten phase containing $\mathrm{K}, \mathrm{Sn}$ and $\mathrm{O}$ in which the 
$\mathrm{K}_{2} \mathrm{SnO}_{3}$ phase forms and nucleates out. $\mathrm{K}_{2} \mathrm{SnO}_{3}$ is a layered structure itself in which the growth predominantly happens at its ends (at edge sites). The growth direction along the wide d-spacing of $6.54 \AA$ was confirmed in all of the TEM analysis of the as-synthesized samples. Availability of $\mathrm{K}^{+}$and $\mathrm{OH}^{-}$ions make the edge, where nucleus is in contact with molten $\mathrm{K}_{2} \mathrm{SnO}_{3}$, is the only active edge that nucleus can propagate to form nanowire.

$\mathrm{SnO}_{2}+2 \mathrm{~K}^{+}+2 \mathrm{OH}^{-} \rightarrow \mathrm{K}_{2} \mathrm{SnO}_{3}+\mathrm{H}_{2} \mathrm{O}$

(5.2.2) $\Delta \mathrm{G}_{2,900}$

Under thermal heating, the reaction (1) can be revised as shown in reaction (2). Under thermal heating up to $900^{\circ} \mathrm{C}, \mathrm{KOH}$ also became molten into free moving $\mathrm{K}^{+}$and $\mathrm{OH}^{-}$ions. The same growth mechanism can be applied in case of thermal heating. Gibbs free energy of $\mathrm{K}_{2} \mathrm{SnO}_{3}$ is not available yet. However, by assuming that the Gibbs free energy of $\mathrm{K}_{2} \mathrm{SnO}_{3}$ is a specific value of $\mathrm{X}_{\mathrm{KSO}}$, then we could calculate Gibbs free energy change of above reactions at $900^{\circ} \mathrm{C} . \Delta \mathrm{G}_{1,900}$ and $\Delta \mathrm{G}_{2,900}$ are of $\mathrm{X}_{\mathrm{KSO}}+256.45$ and $\mathrm{X}_{\mathrm{KSO}}+432.84 \mathrm{~kJ}$. The difference of $176.39 \mathrm{~kJ}$ in Gibbs free energy indicates that presence of plasma makes the reaction (1) more spontaneous than reaction (2). Indeed, fast nanowire growth rate of $1 \mu \mathrm{m} / \mathrm{min}$ is observed with plasma heating while thermal heating resulted in slow nanowire growth rate of $0.04 \mu \mathrm{m} / \mathrm{min}$. As a main specie of atmospheric plasma, highly active oxygen radicals ${ }^{113 \mathrm{~b}}$ with $\mathrm{K}^{+}, \mathrm{OH}^{-}$ions diffuse quickly into tin oxide structure to form $\mathrm{K}_{2} \mathrm{SnO}_{3}$. Fast diffusion of oxygen radicals, $\mathrm{K}^{+}$, and $\mathrm{OH}^{-}$ions results in fast growth rate of nanowires in plasma heating.

$$
\mathrm{SnO}+2 \mathrm{~K}^{+}+2 \mathrm{OH}^{-}+\mathrm{O} \rightarrow \mathrm{K}_{2} \mathrm{SnO}_{3}+\mathrm{H}_{2} \mathrm{O}
$$

In another experiment, tin mono-oxide is used as starting material. The reaction occurs as reaction (3). The Gibbs free energy $\left(\Delta \mathrm{G}_{3,900}\right)$ is calculated to be $\mathrm{X}_{\mathrm{KSO}}+87.75 \mathrm{~kJ}$ 
which is $168.70 \mathrm{~kJ}$ lower than that of reaction (1) where tin oxide is used. As expected, the use of tin mono-oxide has increased the nanowire growth rate up to $40 \mu \mathrm{m} / \mathrm{min}$ that is 40 times faster than the use of tin oxide with plasma heating and 1000 time faster than the use of tin oxide with thermal heating.

$\mathrm{Sn}+2 \mathrm{~K}^{+}+2 \mathrm{OH}^{-}+2 \mathrm{O} \rightarrow \mathrm{K}_{2} \mathrm{SnO}_{3}+\mathrm{H}_{2} \mathrm{O}$

The use of tin metal as starting material made a big difference. Tin metal can react directly with $\mathrm{KOH}$ to form $\mathrm{K}_{2} \mathrm{SnO}_{3}$ nanowires as reaction (4). Tin metal can also get oxidized to tin mono oxides, tin oxides and then these oxides react with $\mathrm{KOH}$ to form $\mathrm{K}_{2} \mathrm{SnO}_{3}$ nanowires. Therefore, random nanowire lengths are observed as tin mono-oxides tend to form long nanowire while tin oxides produce short nanowires. Several resulting tin mono-oxide or tin oxide particles, did not get in contact with $\mathrm{KOH}$ and are observed as particles in any experiments using tin metal as starting material.

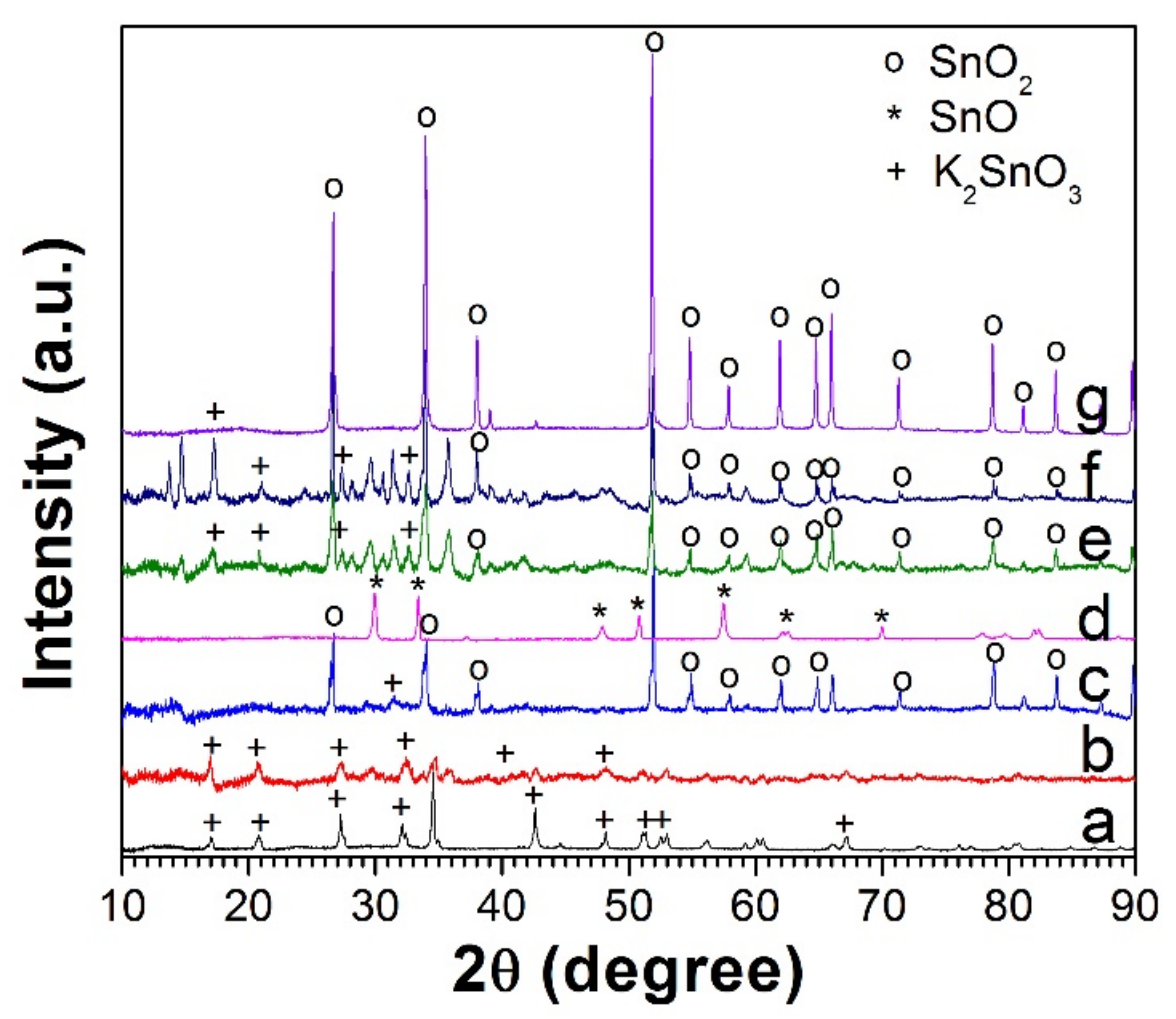


Figure 5.2.3: XRD pattern of a) $\mathrm{K}_{2} \mathrm{SnO}_{3}$ particle precursor, b) as-synthesized nanowires using $\mathrm{K}_{2} \mathrm{SnO}_{3}$ particles, c) as-synthesized nanowires using tin mono oxide particles, d) tin mono oxide particle precursor, e) as-synthesized nanowires using tin oxide particles for 10 seconds exposure time, f) as-synthesized nanowires using tin oxide particles for 60 seconds exposure time, g) $\mathrm{SnO}_{2}$ particle precursor

The structure of as-synthesized nanowires using different starting materials for tin is investigated to understand the transformation of tin-based particle precursor to $\mathrm{K}_{2} \mathrm{SnO}_{3}$ nanowires. Figure 5.2.3 shows the XRD pattern of as-synthesized nanowire samples using tin oxide, tin mono-oxide and $\mathrm{K}_{2} \mathrm{SnO}_{3}$ particles. Clearly, the nanowires prepared using $\mathrm{K}_{2} \mathrm{SnO}_{3}$ particles exhibit the same peaks of $\mathrm{K}_{2} \mathrm{SnO}_{3}$ phase as shown in Figure 5.2.3a and b. Peaks of $\mathrm{K}_{2} \mathrm{SnO}_{3}$ appear in the samples prepared using tin oxide particles after 10 seconds of exposure time (see Figure 5.2.3e). As the exposure time increased to 60 seconds, the intensity of these peaks increase significantly as shown in Figure 5.2.3f. In other experiment with $\mathrm{K}_{2} \mathrm{SnO}_{3}$ particles as precursor, $\mathrm{K}_{2} \mathrm{SnO}_{3}$ remains the same phase but the morphology changes from particle form to nanowire form (Figure 5.2.3a, b and Figure 5.1.7e). The formation $\mathrm{K}_{2} \mathrm{SnO}_{3}$ nanowires using $\mathrm{K}_{2} \mathrm{SnO}_{3}$ particles will be investigated for more detail in the following chapter. The use of tin mono oxide as precursor does not show the formation of $\mathrm{K}_{2} \mathrm{SnO}_{3}$. As shown in Figure 5.1.7d, the long nanowires prepared using tin mono oxide probably experience high temperature gradient and transform directly to tin oxide phase under plasma exposure. This is advantageous as it can further increase the production capacity of tin oxide nanowires using a roll-to-roll setup and expected to introduce a new commercial production of tin oxide nanowire powder for practical applications such as lithium ion batteries, solar cells. 


\subsection{Porous tin oxide as anodes for lithium ion battery application}

Availability of porous tin oxide nanowires in large quantities should be very interesting for prototyping applications in batteries, gas sensors and catalysts ${ }^{114}$. For lithium ion battery application, tin and its oxides are one of the most important anode materials due to their highest volumetric capacity and decent gravimetric capacity of 7.25 $\mathrm{Ah} \mathrm{cm}^{-3}$ and $994 \mathrm{mAh} \mathrm{g}^{-1}$ for tin, $5.47 \mathrm{Ah} \mathrm{cm}^{-3}$ and $781 \mathrm{mAh} \mathrm{g}^{-1}$ for tin oxide, respectively ${ }^{115}$. Moreover, nanowire structures are also known to provide fast charge transport and stress accommodation for lithium ion batteries. In spite of these advantages, tin oxide has been shown to suffer from two major challenges. In addition to mechanical degradation due to volume change (about $250 \%)^{116}$, tin oxide also suffers from chemical degradation, i.e., pulverization of tin oxide into tin and non-conducting $\mathrm{Li}_{2} \mathrm{O}$ phase. Typically, tin and tin oxide based materials suffer from large irreversible capacity loss in first few cycles and continue to fade in capacity with cycling. Recently, Meduri et al. reported that tin nanocluster on tin oxide nanowires could accommodate the volume expansion and increase the conductivity which is resulted in high reversible capacity of

$814 \mathrm{mAh} \mathrm{g}^{-1}$ after 100 cycles $^{5 \mathrm{~b}}$. Based on this concept, the resulting nanowire powders from our synthesis have been treated and tested in lithium ion battery anodes. 

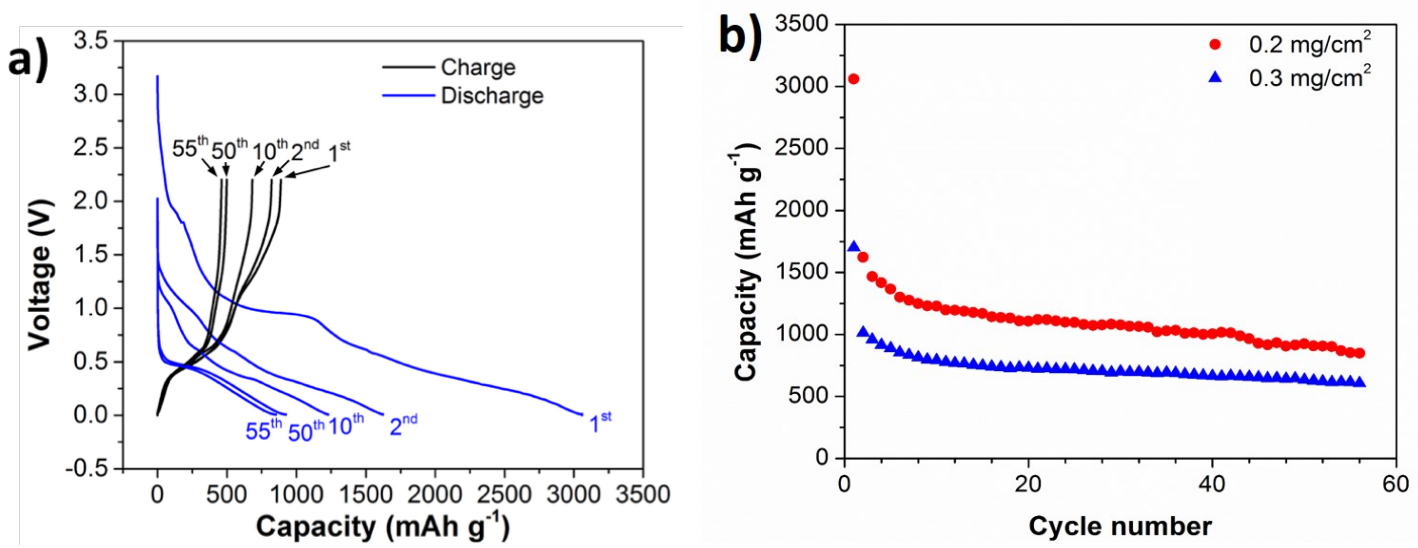

Figure 5.3.1. Galvanostatic curves a) and discharge capacity vs. cycle number b) of tin nanoclusters covered tin oxide nanowires at current density of $100 \mathrm{~mA} / \mathrm{g}$

The resulting tin oxide nanowire powders from our plasma oxidation technique are partially reduced in a hydrogen microwave plasma reactor to form tin nanocluster on the surface of tin oxide nanowires. The tin nanocluster covered tin oxide nanowires are used as active materials for battery testing. To confirm the durability of the tin oxide nanowire electrodes, all of the electrodes are charged and discharged in the voltage range of $0.005-2.2 \mathrm{~V}$ at a current density of $100 \mathrm{~mA} / \mathrm{g}$ (Figure 5.3.1). The high initial capacity of $1700 \mathrm{mAh} \mathrm{g}^{-1}$ is due to the decomposition of electrolyte to form solid electrolyte layer, the reduction of tin oxide to tin, and the insertion of $\mathrm{Li}$ ion into tin oxide and tin to form $\mathrm{Li}_{4.4} \mathrm{Sn}$ alloy. The capacity decreases in first few cycles and remained stable at $614 \mathrm{mAh}$ $\mathrm{g}^{-1}$ after 55 cycles. Another set of experiments with lower loading of $0.2 \mathrm{mg} / \mathrm{cm}^{2}$ shows exceptional high initial capacity of $3059 \mathrm{mAh} \mathrm{g}^{-1}$. The capacity drops quickly in the second cycle to $1623 \mathrm{mAh} \mathrm{g}^{-1}$ and remains stable at $848 \mathrm{mAh} \mathrm{g}^{-1}$ after 55 cycles at the same current density of $100 \mathrm{~mA} / \mathrm{g}$. This is one of the highest capacity retentions obtained for tin oxide. We believe that this high capacity is derived from (1) porous structure of tin oxide which provide high electrode-electrolyte contact area to accommodate the volume 
change during cycling and to provide pathways for fast transportation of lithium ions and easy accessibility of electrolytes ${ }^{117}$; (2) lower loading leading to minimum delamination which has been reported as a major issue with tin oxide nanowires ${ }^{118}$.

\subsection{Generic solvo plasma production of metal oxide nanowires}

It is important to demonstrate that the solvo-plasma are generic for synthesizing most of the metal oxide nanowires. Several oxides of high melting point metals such as $\mathrm{Mn}, \mathrm{Co}, \mathrm{W}$, and Ti have been successfully synthesized using solvo-plasma ${ }^{72}$. The previous section has shown the synthesis of oxide of tin as low melting point metal. To confirm that solvo-plasma will work for most of the oxides of both low melting point and high melting point metals, the synthesis of zinc oxide nanowires is performed using solvo plasma technique. Zinc metal particles $(1 \div 5 \mu \mathrm{m}$ size, Atlantic Equipment Engineer, Inc) mixed with $\mathrm{KCl}$ and water is added to make the precursor paste. As shown in Figure 5.4.1, zinc oxide nanowires were obtained at about $200-400 \mathrm{~nm}$ in diameter and 5 microns in length. The TEM image shows the interplannar spacing of $0.51 \mathrm{~nm}$ which is well matched with the d-spacing of (100) plane of zinc oxide. All of the peaks from zinc oxide sample corresponded to wurtzite hexagonal zinc oxide ${ }^{82}$ as shown in Figure 5.4.1 c. The wurtzite hexagonal zinc oxide was also confirmed by Raman spectrum in Figure 5.4.1 d. Peaks at 334,438 and $578 \mathrm{~cm}^{-1}$ were attributed to $E_{21}, E_{2 h}$ and $E_{1}$ modes, respectively ${ }^{119}$. The observation of direct formation of zinc oxide nanowires from zinc metal particles without any intermediates is as same as the case of tungsten, manganese, and cobalt ${ }^{72}$. 

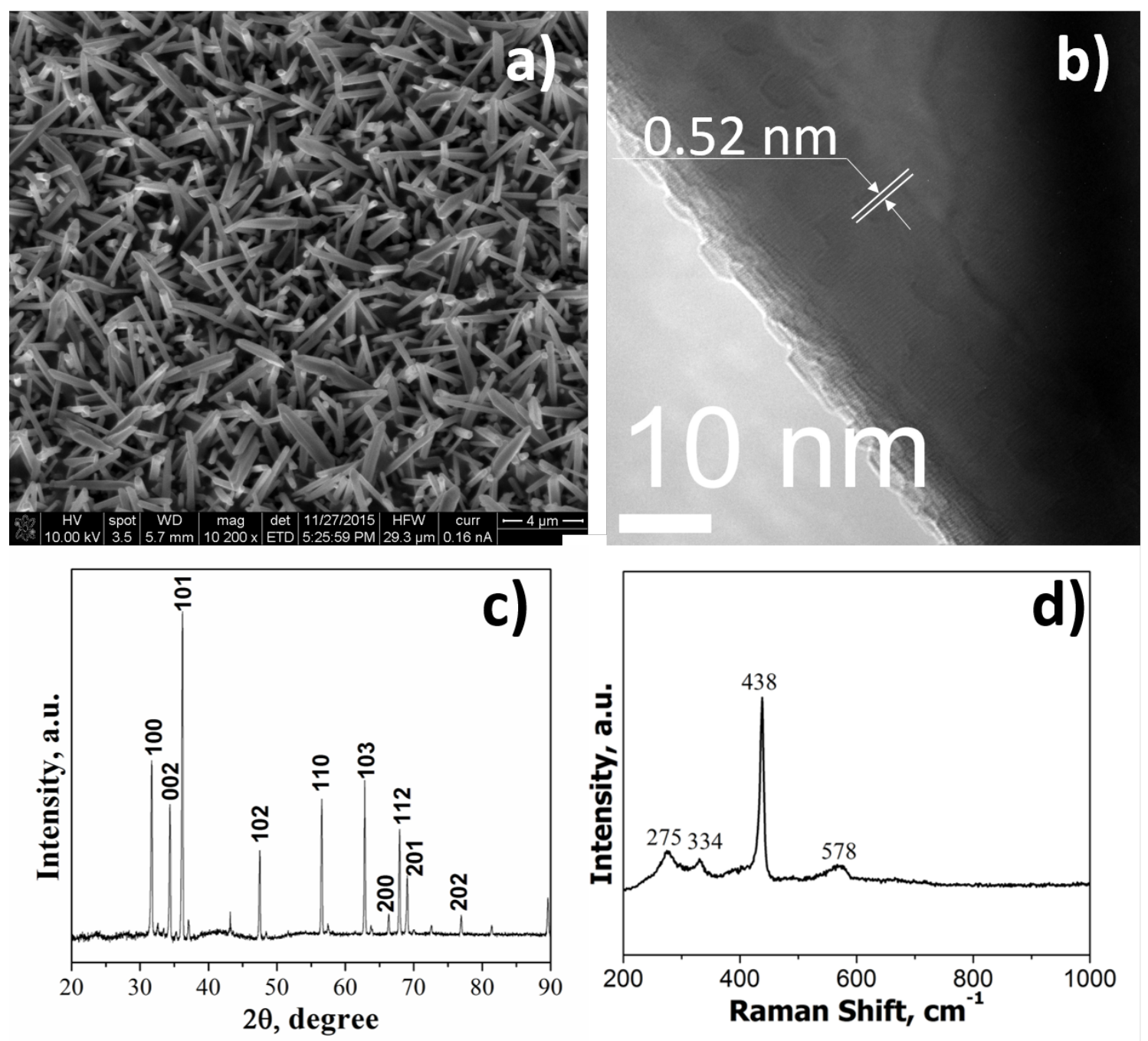

Figure 5.4.1: SEM image a), TEM image b), XRD pattern c) and Raman spectrum c) of resulting zinc oxide nanowires prepared using solvo plasma oxidation

The plasma oxidation experiments using titanium, cobalt, manganese, tungsten, zinc and tin oxide mixed with alkali compounds such as $\mathrm{KCl}$ or $\mathrm{KOH}$ for complete conversion of spherical particles into nanowires are summarized in Table 1. The choice of foil/substrate depended on the type of metal/metal oxide used. The high melting point metals such as titanium, cobalt, manganese, and tungsten required high temperature to be melted and a highly heat conductive foil such as their metal foil or graphite was needed. 
On the other hand, quartz slide could be used for case of tin or zinc where the contamination from foil avoided.

Table 5.5.1: Summary of the experimental conditions used for the NW production

\begin{tabular}{|c|l|l|c|l|l|}
\hline System & $\begin{array}{l}\text { Raw powder } \\
\text { used }\end{array}$ & $\begin{array}{l}\text { Substrate } \\
\text { used }\end{array}$ & $\begin{array}{c}\text { Alkali } \\
\text { reagent }\end{array}$ & $\begin{array}{l}\text { Experimental } \\
\text { condition }\end{array}$ & Results \\
\hline $\mathrm{Ti}$ & $\mathrm{TiO}_{2}$, anatase & $\begin{array}{l}\mathrm{Ti} \text { or } \\
\text { Graphite }\end{array}$ & $\mathrm{KCl}$ & $\begin{array}{l}12 \mathrm{lpm} \text { air, 800- } \\
900 \mathrm{~W}, 2-3 \mathrm{~min}\end{array}$ & Titanate NW array \\
\hline $\mathrm{Co}$ & $\begin{array}{l}\mathrm{Co}_{3} \mathrm{O}_{4}, \\
<50 \mathrm{~nm}\end{array}$ & $\mathrm{Co}$ & $\mathrm{KCl}$ & Same as above & Long bent NWs \\
\hline $\mathrm{Mn}$ & $\mathrm{Mn},<10 \mu \mathrm{m}$ & Graphite & $\mathrm{KCl}$ & Same as above & $\begin{array}{l}\text { Long, beaded and bent } \\
\text { NWs }\end{array}$ \\
\hline $\mathrm{W}$ & $\mathrm{W}, \sim 1 \mu \mathrm{m}$ & Graphite & $\mathrm{KCl}$ & Same as above & Straight cylindrical rod \\
\hline $\mathrm{Sn}$ & $\begin{array}{l}\mathrm{SnO}, \mathrm{SnO} 1- \\
5 \mu \mathrm{m}\end{array}$ & Quartz & $\mathrm{KOH}$ & $\begin{array}{l}12 \text { lpm air, } \\
1.2 \mathrm{~kW}, 1 \text { min }\end{array}$ & $\begin{array}{l}\text { Short stannate NW } \\
\text { array }\end{array}$ \\
\hline $\mathrm{Zn}$ & $\mathrm{Zn}, 1-5 \mu \mathrm{m}$ & Quartz & $\mathrm{KCl}$ & $\begin{array}{l}12 \mathrm{lpm} \text { air, } \\
1.2 \mathrm{~kW}, 1 \mathrm{~min}\end{array}$ & $\begin{array}{l}\text { Short zinc oxide NW } \\
\text { array }\end{array}$ \\
\hline $\mathrm{Si}$ & $\mathrm{Si}, 1-5 \mu \mathrm{m}$ & Quartz & $\mathrm{KOH}$ & $\begin{array}{l}1 \mathrm{~kW}, 1 \mathrm{~min} \\
\text { Silicate nanotubes, } \\
\text { NWs }\end{array}$ \\
\hline
\end{tabular}

\subsection{Scalability of solvo-plasma technique using conveyor belt and fluidized bed} reactor

The scalability of solvo-plasma techniques can be established using a conveyor belt setup. As shown in Figure 5.5.1, the production capacity can be controlled by the thickness of precursor film, width of belt, speed of belt and plasma power. There is one plasma flame in the setup but multiple plasma flames can be used for higher production capacity. In a lab-scale setup, the belt width of 2 inches, the plasma power of 0.8 to 2.5 $\mathrm{kW}$, belt speed of 1 to 2 inches per minutes for exposure time of 30 seconds to 1 minute, the precursor film thickness $100 \mu \mathrm{m}$ to $1 \mathrm{~mm}$. A simple calculation for plasma power of $1.5 \mathrm{~kW}$, belt speed of 1 inch per minute, results in a production rate of 31 to 310 grams/hour for precursor thickness of $100 \mu \mathrm{m}$ to $1 \mathrm{~mm}$ using estimated density of dried 
precursor film of $4 \mathrm{~g} / \mathrm{cm}^{3}$.
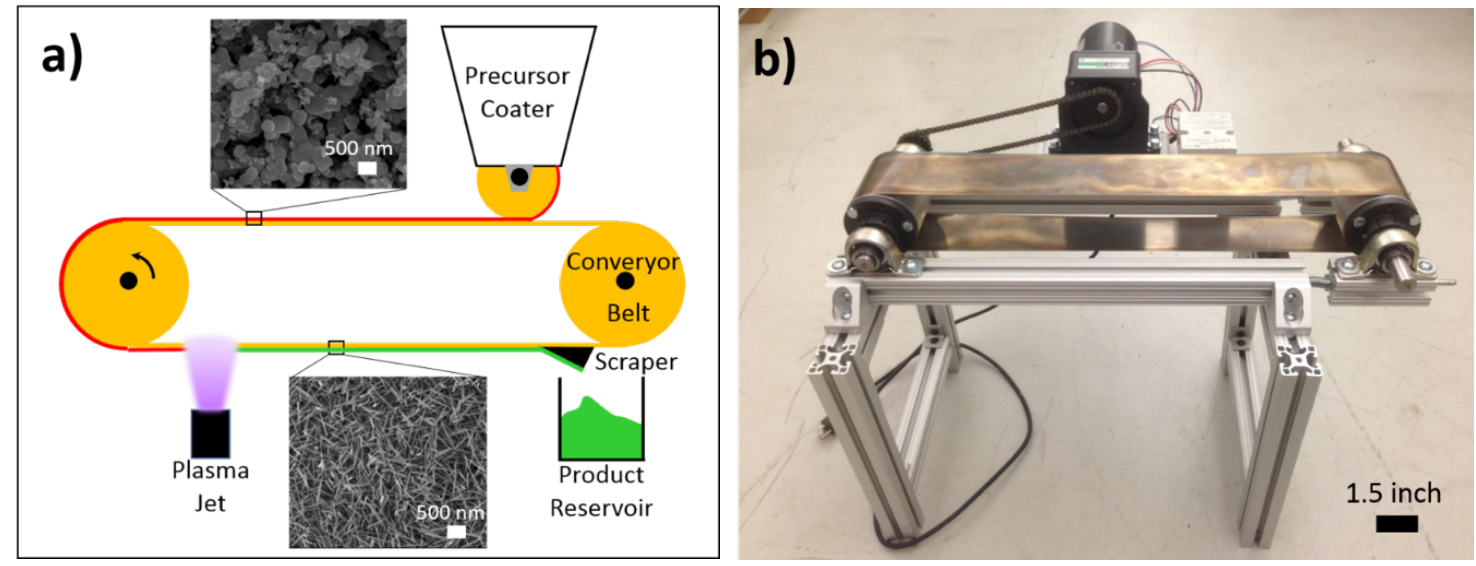

Figure 5.5.1: a) Roll-to-roll setup for commercial production of tin oxide nanowire powder; b) A photo of lab-scale conveyor belt

Experiments were also conducted to understand the solvo-plasma in gas phase without using any support or substrate. The synthesis of titanate and stannate nanowires was performed in the downstream atmospheric plasma reactor as described in chapter 3 . $\mathrm{K}_{2} \mathrm{O}$. $\mathrm{TiO}_{2}$ particles were used for titanate nanowire synthesis while stannate particles and mixture of $\mathrm{SnO}_{2}$ particles and $\mathrm{KOH}$ were used for stannate nanowire synthesis. As shown in Figure 5.5.2, titanate nanowires of about $30 \mu \mathrm{m}$ in length and about $200 \mathrm{~nm}$ in diameter were obtained. The stannate nanowires were thinner and shorter. This set of preliminary data shows that titanate and stannate partciles were mostly converted to nanowires. The use of mixture of tin oxide particles and $\mathrm{KOH}$ resulted in lower conversion. The low conversion may be due to short residence time and poor mixing of tin oxide particles and $\mathrm{KOH}$. However, the conversion and nanowire morphology can be controlled by several parameters such as plasma power, total gas flow, gas composition, and mass ratio of tin oxide particles and $\mathrm{KOH}$. The downstream plasma reactor offers very short residence time of about 0.01 second. Thus, it is possible to produce these 
nanowires in fluidized bed reactor (see chapter 3 ) where the residence time can be in order of seconds or higher due to fluidization of starting particles.
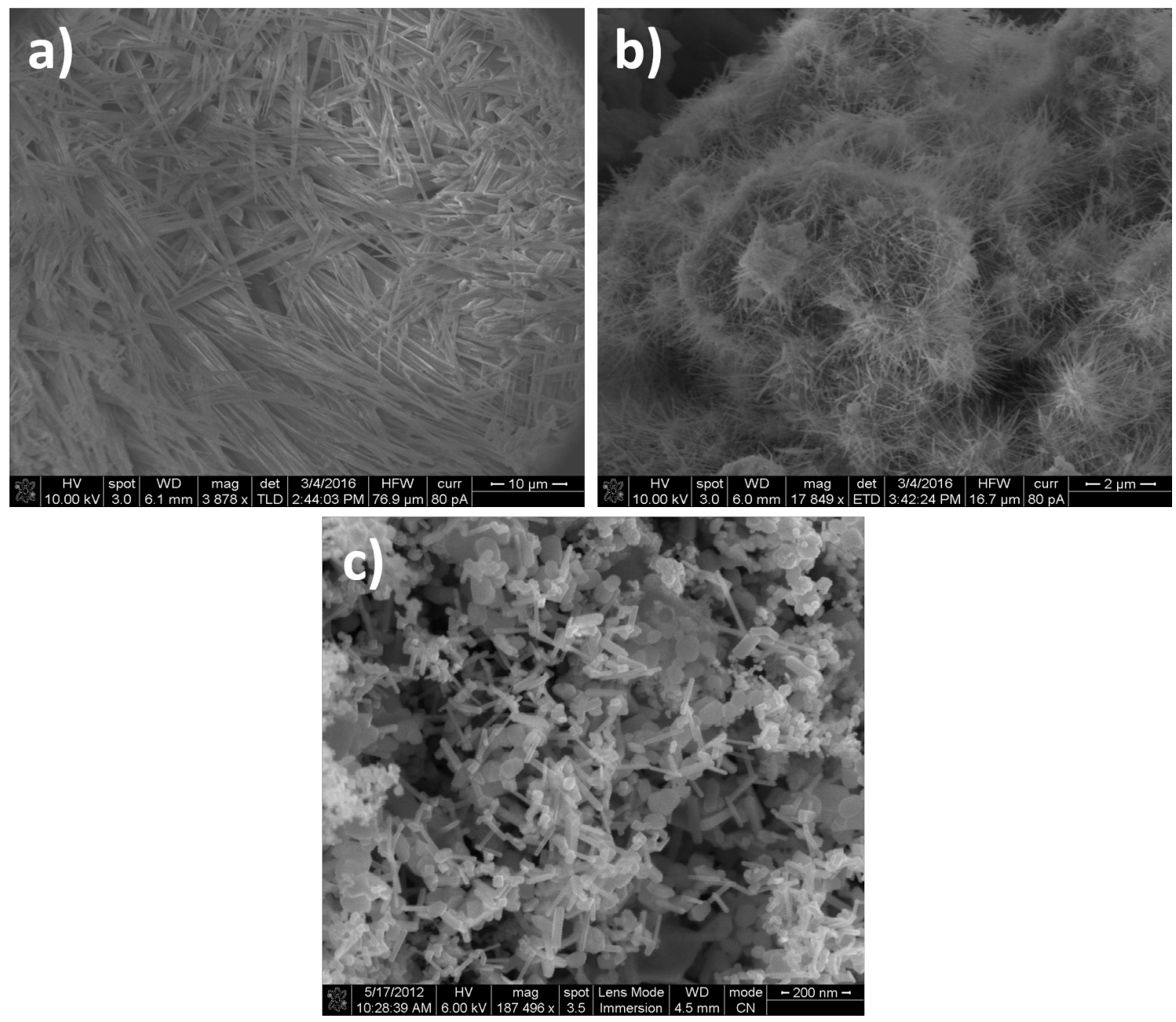

Figure 5.5.2: SEM images of as-synthesized nanowires produced using the downstream atmospheric microwave plasma reactor. a) titanate, b) stannate, and c) stannate nanowires produced using $\mathrm{K}_{2} \mathrm{O}$. $\mathrm{TiO}_{2}$ powder, $\mathrm{K}_{2} \mathrm{SnO}_{3}$ powder and mixture of $\mathrm{SnO}_{2}$ particles and $\mathrm{KOH}$, respectively.

\subsection{Summary}

In summary, a simple concept for fast, scalable method is presented and studied for converting tin oxide particles to tin oxide nanowires by mixing it with potassium 
hydroxide and exposing to an atmospheric microwave plasma reactor. The reaction time scales have been found to be on the order of few tens of seconds to a minute which makes this technique highly scalable. A fast nanowire growth rate of $1 \mu \mathrm{m} / \mathrm{min}$ was observed with plasma heating which is 25 time higher than thermal heating. The use of tin monooxide as precursor could bring the growth rate up to $40 \mu \mathrm{m} / \mathrm{min}$. A systematic set of experiments revealed the presence of intermediate phase $\left(\mathrm{K}_{2} \mathrm{SnO}_{3} \cdot \mathrm{H}_{2} \mathrm{O}\right)$ formation which could be held responsible for the observed one-dimensional growth. Further acid wash and annealing of $\mathrm{K}_{2} \mathrm{SnO}_{3} \cdot \mathrm{H}_{2} \mathrm{O}$ allowed for formation of porous tin oxide nanowires. The resulting porous tin oxide nanowires showed a high reversible capacity of $848 \mathrm{mAh} \mathrm{g}^{-1}$ after 55 cycles at a current density of $100 \mathrm{~mA} / \mathrm{g}$.

The solvo-plasma technique seems to be generic scheme for synthesizing most of metal oxides including oxides of low and high melting point metal such as titanium, cobalt, manganese, tungsten, zinc and tin oxide. Solvo-plasma is fast, scalable method which is an ideal replacement for several liquid phase techniques such as hydrothermal, precipitation, electrochemical syntheses. A production capacity of 300 grams per hour can be obtained by a simple lab-scale roll-to-roll setup. The production capacity can be increased further by using fluidized bed reactor. 


\section{CHAPTER VI}

\section{PROTECTIVE COATING IN NANOWIRE BASED ANODES}

\subsection{Introduction}

In this chapter, challenges associated with nanowire based materials for lithium ion battery application such as volume expansion, and initial capacity loss will be investigated. Tin-based materials have attracted great deal of attention for anodes in lithium-ion batteries (LIBs) due to their high theoretical capacities of $781 \mathrm{mAhg}^{-1}, 991$ $\mathrm{mAhg}^{-1}$ for case of $\mathrm{SnO}_{2}$ and $\mathrm{Sn}$, respectively ${ }^{115}$. In particular, tin oxide $\left(\mathrm{SnO}_{2}\right)$ has been considered as a promising candidate that can substitute for commonly used graphite electrodes. However, $\mathrm{SnO}_{2}$ based anodes suffer from two major drawbacks: (i) capacity fading due to their volume expansion during cycling ${ }^{116,}{ }^{120}$ leading to mechanical degradation, pulverization of electrode materials with chemical degradation and (ii) initial capacity loss resulting from the formation of insulating $-\mathrm{Li}_{2} \mathrm{O}^{121}$, and solid electrolyte interface (SEI) ${ }^{122}$.

Several approaches have focused on improving the capacity retention by lowering the pulverization of $\mathrm{SnO}_{2}$ and mechanical degradation due to volume expansion. For example, Ding et al. ${ }^{123}$ reported that a capacity of $773.7 \mathrm{mAhg}^{-1}$ could retain after 100 
cycles by using a nanocomposite $\mathrm{SnO}_{2}$-Se thin film. Similarly, a study by $\mathrm{Yu}$ et al. has shown that a novel composite of $\mathrm{Li}_{2} \mathrm{O}-\mathrm{CuO}-\mathrm{SnO}_{2}$ exhibited a capacity of $1158 \mathrm{mAhg}^{-1}$ at $0.5 \mathrm{C}$ and $525 \mathrm{mAhg}^{-1}$ at $8 \mathrm{C}$ after 100 cycles $^{124}$. Xia et al. have reported that composites of $\mathrm{SnO}_{2}$ nanoparticles and carbon nanofiber showed a capacity retention of $383 \mathrm{mAhg}^{-1}$ after 30 cycles at the current density of $100 \mathrm{mAg}^{-1}{ }^{125}$. Mei et al. ${ }^{117}$ has shown a reversible capacity of $810 \mathrm{mAhg}^{-1}$ after 50 cycles for the hybrid electrode consisting of porous $\mathrm{Co}-\mathrm{SnO}_{2}$ spheres.

Nanowire morphology offer conducting pathways and short length scales for lithium ion diffusion. However for $\mathrm{SnO}_{2}$ nanowires $\left(\mathrm{SnO}_{2} \mathrm{NWs}\right)$, the chemical degradation through $\mathrm{Li}_{2} \mathrm{O}+\mathrm{Sn}$ formation during lithium alloying/de-alloying will result in quick degradation of morphology within first five cycles leading to severe capacity degradation to around $300 \mathrm{mAhg}^{-1}{ }^{126}$. Several approaches have been reported in the literature to improve capacity retention of $\mathrm{SnO}_{2} \mathrm{NW}$-based anodes. For instance, Kim et al. ${ }^{127}$ has synthesized coaxial $\mathrm{SnO}_{2}-\mathrm{In}_{2} \mathrm{O}_{3}$ heterostructured nanowires and shown a high capacity retention of $700 \mathrm{mAhg}^{-1}$ after 10 cycles due to high electronic conductivity of heterostructured nanowires by incorporation of $\mathrm{Sn}$ into $\operatorname{In}_{2} \mathrm{O}_{3}$ shell. In another study, a binder-free $\mathrm{SnO}_{2} \mathrm{NWs}$, grown directly on current collector, has been shown to deliver a capacity of $600 \mathrm{mAhg}^{-1}$ after 30 cycles at the current density of $782 \mathrm{mAg}^{-1}$ (1C) but using only a small voltage range of $0.0-1.2 \mathrm{~V}^{128}$. Recently, Zhang et al. ${ }^{129}$ has shown that an increase of 3-4 orders of magnitude in conductivity of $\mathrm{SnO}_{2} \mathrm{NWs}$ could be obtained by coating with a layer of carbon which helped with rate perfomrmance. On the other hand, Sn nanocluster covering $\mathrm{SnO}_{2}$ NWs have exhibited an exceptional capacity of 814 $\mathrm{mAhg}^{-1}$ after 100 cycles $^{5 \mathrm{~b}}$. However, none of these approaches has addressed the 
reduction of the irreversible capacity losses that results in severe capacity fading in the first few cycles.

More recently, there has been increased interest in using protective layers on active materials to reduce the irreversible capacity loss. A key requirement for good protective coating is to have poor electrical conductivity and high Li ion conductivity. These approaches have focused on using wet chemical methods to form titania-based protective coatings on $\mathrm{SnO}_{2}$ particles ${ }^{130}, \mathrm{SnO}_{2}$ nanofibers ${ }^{131}$ and $\mathrm{SnO}_{2}$ nanotubes ${ }^{132}$. These methods, however, require multiple complex steps for making the protective coating and often result in incomplete coverage of the tin oxide leading to pin hole in the protective layer $^{130}$. Recently, atomic layer deposition (ALD) has been introduced as an effective technique for depositing uniform layers on nanostructures ${ }^{133}$. Although, the use of titania-based materials as protective coatings has been shown to improve electrochemical performance, several fundamental questions as to how these materials act as protective coating, mechanism of SEI formation and factors determining the lowering of irreversible capacity loss in the first cycle, still need to be addressed and understood.

However, there have been few studies performed to understand the stability of thin coatings and their influence on the morphological stability of nanowires. In order to gain fundamental insight, binder-free $\mathrm{SnO}_{2} \mathrm{NW}$ arrays coated with thin protective layers are studied here in this work.

\subsection{Experimental section}

$\mathrm{SnO}_{2} \mathrm{NW}$ arrays are synthesized on a variety of substrates including stainless steel (SS) (Alfa Aesar) using a reactive vapor transport of tin in lean oxygen conditions ( $5 \mathrm{sccm} \mathrm{O}_{2}$ and $350 \mathrm{sccm} \mathrm{H}_{2}$ ) at 1 Torr. The detailed experiment procedure has been 
described earlier ${ }^{134}$. Briefly, the source material is placed in an alumina coated tungsten boat heater. The Sn metal vapor gets oxidized on the substrate and leads to the growth of vertical nanowire arrays in a self-catalyzed fashion. The as-synthesized $\mathrm{SnO}_{2} \mathrm{NWs}$ on $\mathrm{SS}$ are coated with titania or alumina using an ALD (Cambridge Nanotech) at $250^{\circ} \mathrm{C}$ with titanium tetra isopropoxide or trimethyl aluminum, respectively and water as precursors. In each cycle, a pulse duration of 0.2 seconds, and nitrogen flow rate of $20 \mathrm{sccm}$ are used. The samples are characterized using a scanning electron microscope (FEI Nova 600), Xray diffraction (Bruker D8 Discovery with $\mathrm{Cu} \mathrm{K} \alpha$ radiation), and a transmission electron microscope (Tecnai F20 FEI TEM operating at $200 \mathrm{kV}$ ).

The electrochemical measurements are performed using CR2032 coin-type cells assembled in a dry argon-filled glove box. The test cell consists of a working anode electrode titania coated $\mathrm{SnO}_{2} \mathrm{NWs}$ and counter electrode of lithium metal. The electrodes are separated by two pieces of glass fiber filter (ADVANTEC GB-100R. Toyo Rishi CO., Japan). The electrolyte solution used is $1 \mathrm{M} \mathrm{LiPF} 6^{-}$ethylene carbonate (EC): dimethyl carbonate (DMC) (1:2 by volume). No binders are used. The charge-discharge measurements are carried out using battery tester (16 channel Arbin Instruments, USA). 


\subsection{Results and discussion}

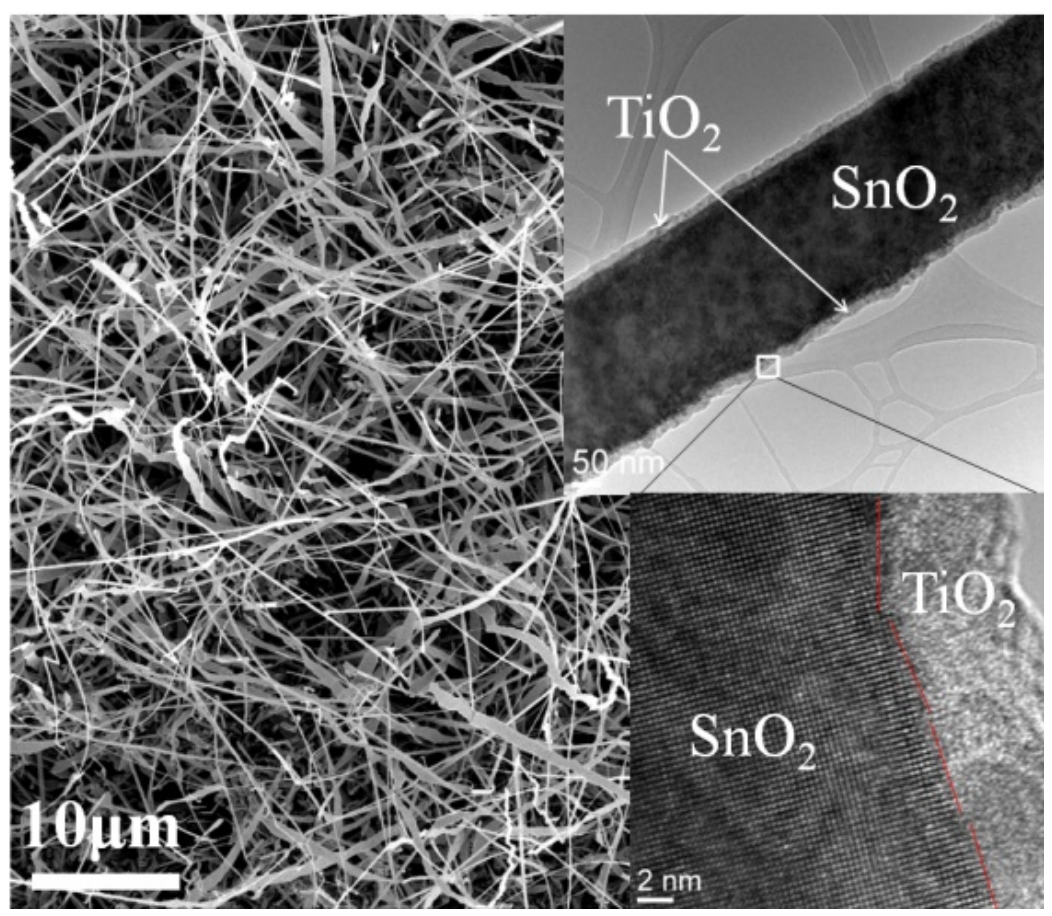

Figure 6.1 SEM and TEM (inset) images of titania-coated $\mathrm{SnO} 2 \mathrm{NWs}$ before cycling

$\mathrm{SnO}_{2} \mathrm{NW}$ arrays are synthesized directly on SS substrate without using any catalyst. As shown in Figure 1, the $\mathrm{SnO}_{2}$ NWs are $\sim 300$ to $1000 \mathrm{~nm}$ in diameter and about tens of microns in length. The as-grown $\mathrm{SnO}_{2} \mathrm{NW}$ arrays on steel substrate are coated with thin layers of either titania or alumina. The TEM image in Figure 6.1 shows the atomic layer deposited $15 \mathrm{~nm}$ thick titania on $\mathrm{SnO}_{2}$ nanowires. The thickness of titania or alumina coating is varied from $15 \mathrm{~nm}$ to $1 \mathrm{~nm}$ in this study.

The lithium intercalation-de-intercalation with tin oxide is expected to proceed according to the following two reactions ${ }^{135}$. The first reaction is typically considered as irreversible and the second on is reversible.

$$
\begin{aligned}
& \mathrm{SnO}_{2}+4 \mathrm{Li}^{+}+4 \mathrm{e}^{-} \rightarrow 2 \mathrm{Li}_{2} \mathrm{O}+\mathrm{Sn} \\
& \mathrm{Sn}+\mathrm{xLi}^{+}+\mathrm{xe}^{-} \leftrightarrow \mathrm{Li}_{\mathrm{x}} \mathrm{Sn}(0 \leq \mathrm{x} \leq 4.4)
\end{aligned}
$$



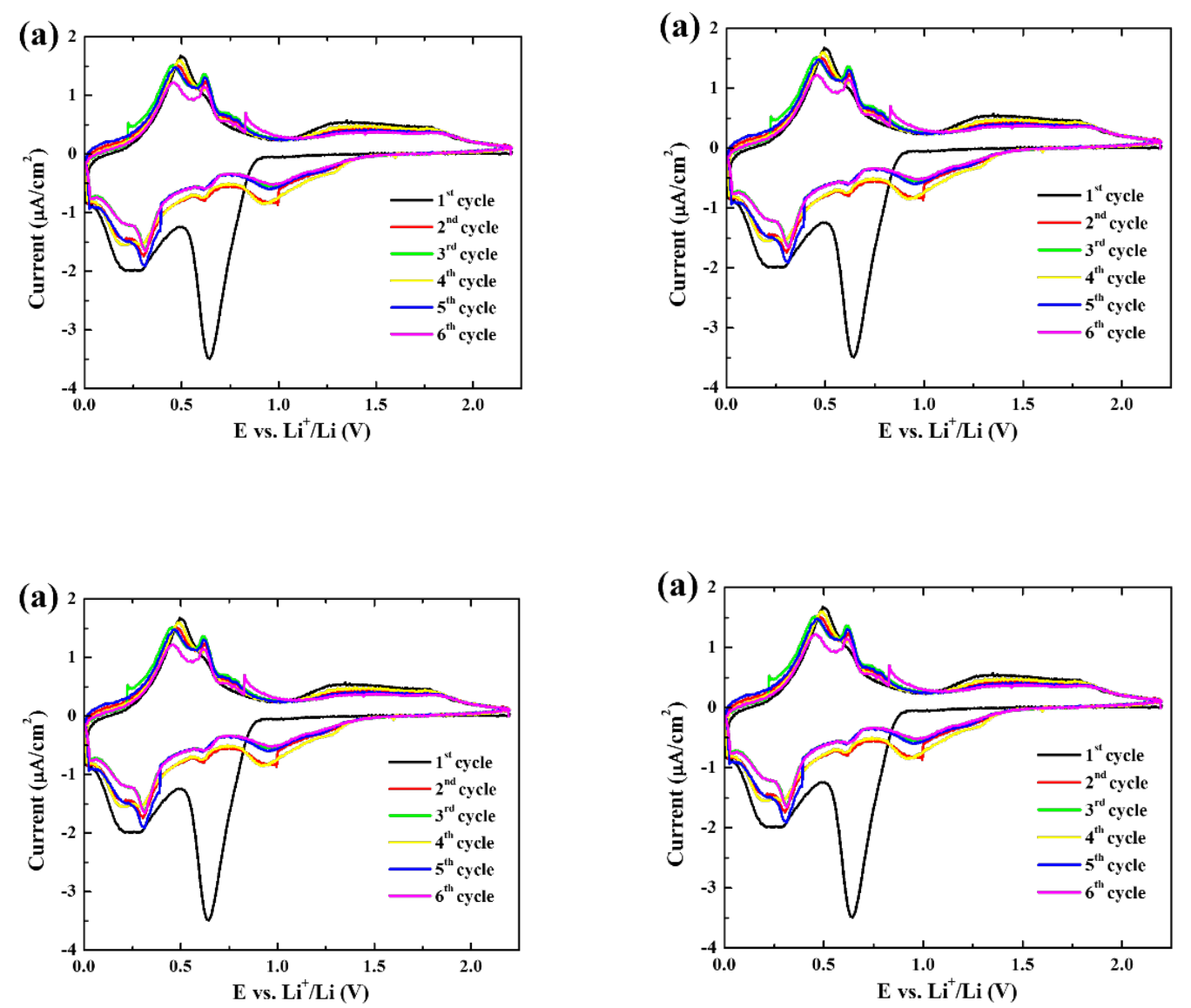

Figure 6.2 a) Cyclic voltammetry of titania-coated $\mathrm{SnO} 2 \mathrm{NWs}$ at the voltage range of 2.2$0.005 \mathrm{~V}$ using scan speed of $5 \mathrm{mV} / \mathrm{min}$. b) Charge-discharge capacity of pure $\mathrm{SnO} 2$ NWs and titania-coated $\mathrm{SnO} 2 \mathrm{NWs}$ at the voltage range of 2.2-0.005 V using current density of $60 \mathrm{~mA} / \mathrm{g}$; c) Comparison of capacity vs. cycle number of $\mathrm{SnO} 2 \mathrm{NWs}$ and titania-coated $\mathrm{SnO} 2 \mathrm{NWs}$ at currents of 60, 700, and $1500 \mathrm{~mA} / \mathrm{g}$; d) Comparison of capacity vs. cycle number of $\mathrm{SnO} 2 \mathrm{NWs}$ and alumina-coated $\mathrm{SnO} 2 \mathrm{NWs}$ at currents of 60,700 , and $1500 \mathrm{~mA} / \mathrm{g}$

Cyclic voltammetry (CV) of pure tin oxide nanowires powders mixed with binders shows peaks corresponding to the above two reactions. Analysis of the CV of tin oxide nanowires coated with protective layers is shown in Figure 6.2 a. In addition to 
alloying peaks at $\sim 0.1-0.3 \mathrm{~V}^{136}$ and dealloying peaks at $\sim 0.5-0.6 \mathrm{~V}^{137}$ seen for pure tin oxide NWs, a sharp cathodic peak at $0.68 \mathrm{~V}$ corresponding to the irreversible reduction of tin oxide to tin is observed ${ }^{138}$. The peak at $0.68 \mathrm{~V}$ is significantly reduced in the subsequent cycles due to the irreversibility of reaction (1). In comparison with CV of pure $\mathrm{SnO}_{2} \mathrm{NWs}$ (Figure 6.3), the peak position is shifted from $0.8 \mathrm{~V}$ to $0.68 \mathrm{~V}$, and could be due to presence of coating layers. The second and the subsequent cycles shows broad cathodic peak at $0.85 \mathrm{~V}$ that could be assigned to formation of an SEI layer ${ }^{136}$. Additional anodic peaks at $0.25 \mathrm{~V}, 0.75 \mathrm{~V}$ and $0.85 \mathrm{~V}$ could be due to delithiation of $\mathrm{Li}_{x} \mathrm{Sn}$ alloy formed by tin cluster coming out on the surface of coatings ${ }^{134}$. These peaks have not been observed in $\mathrm{CV}$ of pure tin oxide nanowires, indicating that $\mathrm{Sn}$ clusters on the surface are formed only when the protective coatings are used.

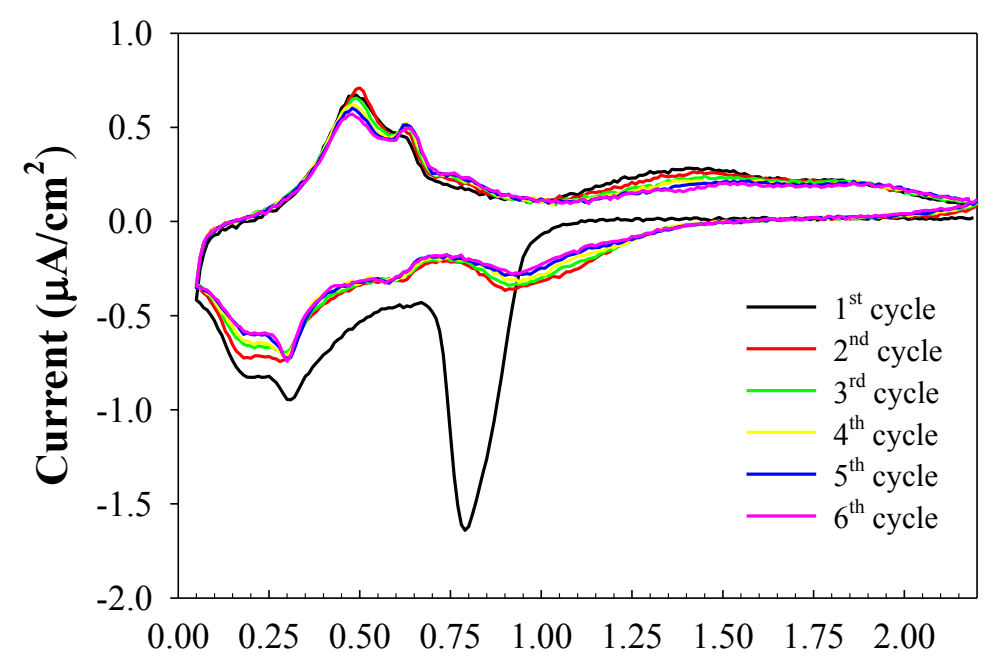

\section{E vs. $\mathbf{L i}^{+} / \mathbf{L i}(\mathrm{V})$}

Figure 6.3: Cyclic voltammetry of $\mathrm{SnO}_{2} \mathrm{NWs}$ at the voltage range of $2.2 \div 0.005 \mathrm{~V}$ using scan speed of $5 \mathrm{mV} / \mathrm{min}$

Figure $6.2 \mathrm{~b}$ shows a comparison of $1^{\text {st }}$ cycle charge-discharge capacity of pure $\mathrm{SnO}_{2} \mathrm{NWs}$ and titania-coated $\mathrm{SnO}_{2} \mathrm{NWs}$ in the voltage range of $0.005-2.2 \mathrm{~V}$ using 
current density of $60 \mathrm{mAg}^{-1}$. The initial discharge capacity of titania-coated $\mathrm{SnO}_{2} \mathrm{NWs}$ is $1705 \mathrm{mAhg}^{-1}$ which is twice theoretical capacity of bulk $\mathrm{SnO}_{2}$. The high initial discharge capacity is believed to be due to reduction of tin oxide to tin and the intercalation of $\mathrm{Li}$ into $\mathrm{SnO}_{2}$ and complete access to all of the tin for forming $\mathrm{Li}_{4.4} \mathrm{Sn}$ alloys as depicted by the plateau at $0.9 \mathrm{~V}$. The first cycle columbic efficiency is $72.6 \%$, which is much higher than that of our pure $\mathrm{SnO}_{2} \mathrm{NWs}$ of $49.5 \%$ (Table 6.1), and also much higher than $60 \%$ previously reported on similar structure of titania-coated $\mathrm{SnO}_{2}$ hollow nanoparticles ${ }^{130}$. To the best of our knowledge, this is the highest and first of its kind value reported for binder-free $\mathrm{SnO}_{2}$-based electrodes. The initial capacity loss due to SEI formation can be calculated based on the overall capacity loss and the capacity loss due to tin oxide reduction ${ }^{130}$. Accordingly, the initial capacity loss due to SEI formation for pure $\mathrm{SnO}_{2}$ NWs is calculated to be $448 \mathrm{mAhg}^{-1}$ while the titania and alumina coated $\mathrm{SnO}_{2} \mathrm{NW}$ arrays electrodes show no initial capacity loss due to SEI formation (Table 6.1). The previous attempt to use lithium titanate as protective coating identified the incomplete coverage as one of the major challenges in reducing irreversible capacity loss ${ }^{130}$. In our case, the full coverage suppressed the formation of SEI in the first cycle. Both titania and alumina coatings minimize the irreversible capacity loss and accounts for the observed increase in the columbic efficiency when compared to uncoated $\mathrm{SnO}_{2}$ NWs. A charge discharge measurement of titania-coated $\mathrm{SnO}_{2} \mathrm{NWs}$ at $1.0 \div 3.0 \mathrm{~V}$ (Figure 6.4) shows a small capacity of $6 \mathrm{mAhg}^{-1}$ which means that all of the observed capacities are mainly from $\mathrm{SnO}_{2} \mathrm{NWs}$. 


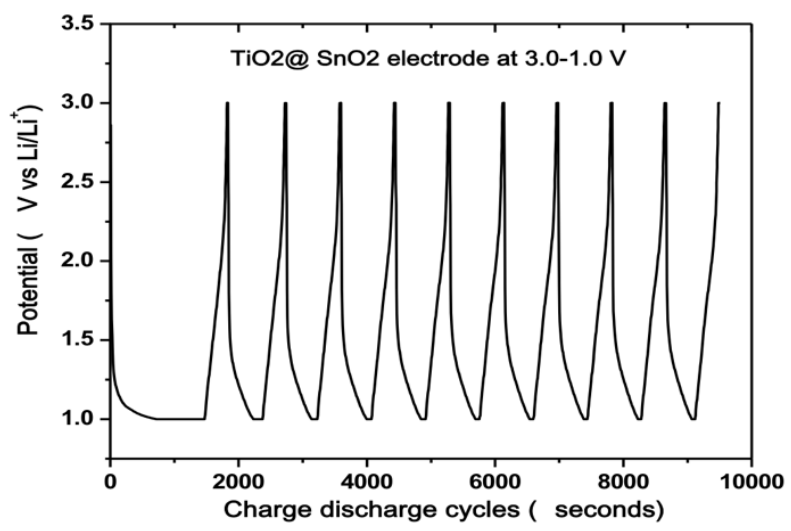

Figure 6.4: The charge-discharge capacities with times vs. voltage profiles of titaniacoated $\mathrm{SnO}_{2} \mathrm{NWs}$ electrode at 3.0-1.0 V

Table 6.1: $1^{\text {st }}$ cycle electrochemical performance comparison of pure $\mathrm{SnO}_{2} \mathrm{NWs}$ and titania-coated $\mathrm{SnO}_{2} \mathrm{NWs}$

\begin{tabular}{llcllll}
\hline Sample & $\begin{array}{l}\text { Discharge } \\
\text { capacity, } \mathrm{mAhg}^{-1}\end{array}$ & $\begin{array}{l}\text { Charge capacity, } \\
\mathrm{mAhg}^{-1}\end{array}$ & $\begin{array}{l}\text { Columbic } \\
\text { efficiency, } \\
\%\end{array}$ & $\begin{array}{l}\text { ICL, } \\
\mathrm{mAhg}^{-1}\end{array}$ & $\begin{array}{l}\text { ICL due to reduction } \\
\text { of tin oxide to tin } \\
\mathrm{mAhg}^{-1}\end{array}$ & $\begin{array}{l}\text { ICL due to SEI } \\
\mathrm{mAhg}^{-1}\end{array}$ \\
\hline $\begin{array}{l}\text { Pure } \quad \mathrm{SnO}_{2} \\
\mathrm{NWs}\end{array}$ & 1680 & 832 & 49.5 & 848 & 400 & 448 \\
$\begin{array}{l}\text { titania- } \\
\text { coated } \mathrm{SnO}_{2}\end{array}$ & 1705 & 1238 & 72.6 & 467 & 520 & - \\
\hline
\end{tabular}

Figure $6.2 \mathrm{c}$ shows the comparison of capacity vs. cycle number of $\mathrm{SnO}_{2} \mathrm{NWs}$ and titania-coated $\mathrm{SnO}_{2}$ NWs at current density of 60,700 , and $1500 \mathrm{mAg}^{-1}$. The pure $\mathrm{SnO}_{2}$ electrode exhibits an initial discharge capacity of $1680 \mathrm{mAhg}^{-1}$ at a current density of $60 \mathrm{mAg}^{-1}$ but fades quickly to $316 \mathrm{mAhg}^{-1}$ after 30 cycles. Coating of $\mathrm{SnO}_{2} \mathrm{NWs}$ with $15 \mathrm{~nm}$ titania resulted in similar initial capacity as the one measured for uncoated $\mathrm{SnO}_{2}$ NWs (1705 mAhg $\left.{ }^{-1}\right)$. However, a significant increase of capacity retention of 634 $\mathrm{mAhg}^{-1}$ after 30 cycles at the same current of $60 \mathrm{mAg}^{-1}$ is observed. Even a thinner 
titania layer of $5 \mathrm{~nm}$ showed higher capacity retention of 767 and $664 \mathrm{mAhg}^{-1}$ at the higher current density of 700 and $1500 \mathrm{mAg}^{-1}$, respectively. Previous studies have showed that amorphous titania has higher lithium diffusivity coefficient, higher rate capacity than those of anatase titania ${ }^{139}$. The use of amorphous titania in our study can result in formation of intermediate phase of $\mathrm{Li}_{\mathrm{x}} \mathrm{TiO}_{2}$ which has been shown to be a good lithium ion conductor ${ }^{139 a}$. All of these supports the fact that coating titania on $\mathrm{SnO}_{2}$ enables the high rate capacity of titania-coated $\mathrm{SnO}_{2} \mathrm{NWs}$ electrodes. Thus, the presence of a uniformly coated titania layer is one of the reason why the high capacity retention is obtained at very high current density. Another reported experiment with alumina as coating layer has shown good performance on cathode materials ${ }^{133}$. In our study, the 1 nm thick alumina-coated $\mathrm{SnO}_{2} \mathrm{NW}$ electrode exhibited a high capacity retention of 725 and $663 \mathrm{mAhg}^{-1}$ at the current density of 700 and $1500 \mathrm{mAg}^{-1}$ after 30 cycles, respectively (Figure 6.2 d). During cycling, there may be formation of $\mathrm{Li}_{2} \mathrm{O}-\mathrm{Al}_{2} \mathrm{O}_{3}$ glass which has high ionic conductivity and low electrical conductivity ${ }^{140}$. The $\mathrm{Li}_{2} \mathrm{O}-\mathrm{Al}_{2} \mathrm{O}_{3}$ glass could serve as a stable solid electrolyte for fast lithium ion diffusion ${ }^{141}$. This is also in agreement with previous report that lithium ion could transport through the electrically insulating layer of alumina and improved the rate capacity ${ }^{142}$. 


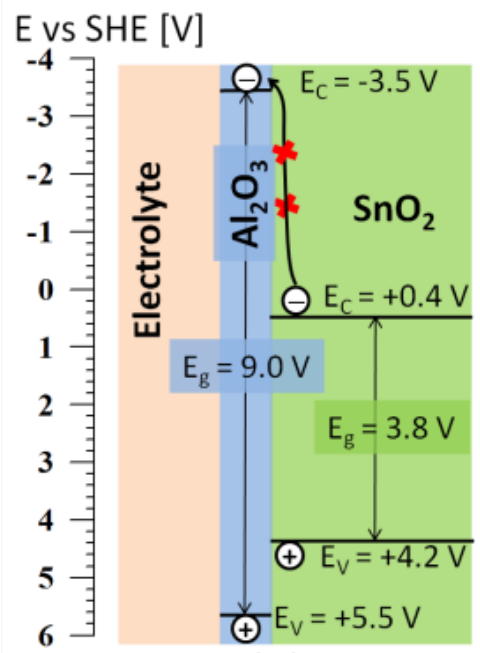

(a)

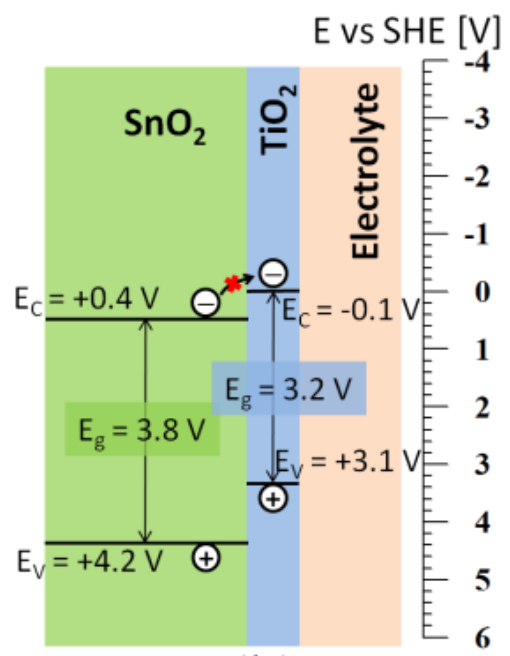

(b)

Figure 6.5: Energy band diagram of $\mathrm{SnO}_{2}$ and $\mathrm{Al}_{2} \mathrm{O}_{3}$ (a), $\mathrm{TiO}_{2}$ (b) with respect to the electrochemical scale. Ev, Ec, and Eg represent valence band maxima, conduction band minima and bandgap, respectively

The lack of SEI formation during first cycle with titania- or alumina-coated $\mathrm{SnO}_{2}$ NWs can be explained by the band edge diagram of $\mathrm{SnO}_{2}$ and coated layers. See Figure 6.5 , the band edge off-set prevents the electron migration from tin oxide to coatings and then to electrolyte. Further poor electrical conductivity and poor electron transport properties of amorphous titania inhibit electron transfer to the electrolyte mitigating SEI formation in the first cycle ${ }^{143}$. Moreover, the band edge off-set created by alumina layer is even larger. No electron migration from tin oxide to electrolyte through alumina is expected as well. Thus, there is no SEI formation in the first cycle. The observation of SEI formation in the second cycle is surprising and will be explained later. 

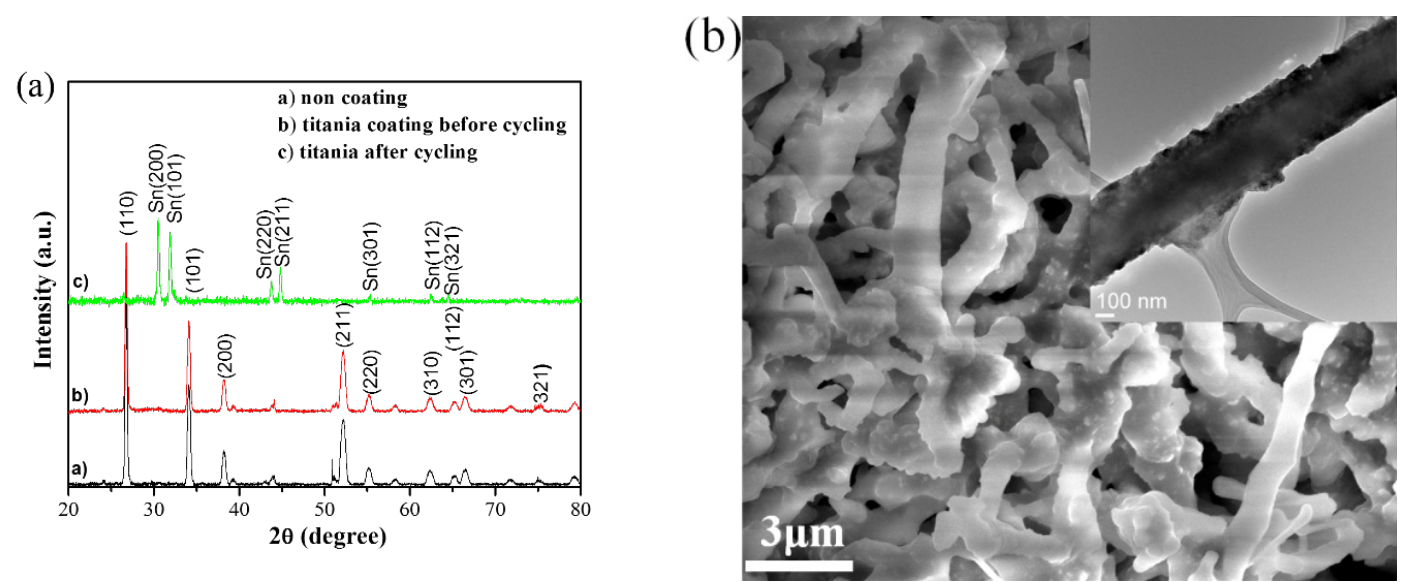

Figure 6.6: The XRD pattern showing no peak of titania due to the amorphous phase, the tin oxides were completely reduced to tin after cycling (a), SEM and TEM (inset) images of titania-coated $\mathrm{SnO} 2 \mathrm{NWs}$ after 100 cycles showing the original morphology (b)

Figure 6.6a shows the XRD patterns of pure $\mathrm{SnO}_{2} \mathrm{NWs}$, and titania-coated $\mathrm{SnO}_{2}$ NWs. All of the peaks of $\mathrm{SnO}_{2} \mathrm{NWs}$ are assigned to pure $\mathrm{SnO}_{2}$ single-phase structure [PDF 00-002-1340], while titania coated $\mathrm{SnO}_{2} \mathrm{NWs}$ shows same phase with low intensity peaks. No peaks corresponding to titania or alumina were observed from tin oxide nanowires coated with thin layers indicating amorphous nature of the coatings. The TEM image in Figure 6.1 also corroborates the formation of an amorphous layer of titania. The XRD patterns also shows that $\mathrm{SnO}_{2} \mathrm{NWs}$ are completely reduced to $\mathrm{Sn}$ after cycling when using titania-coated $\mathrm{SnO}_{2}$ NWs [PDF 03-065-0296]. This is completely expected and similar to other studies using $\mathrm{SnO}_{2}$ electrode ${ }^{126-127}$. However, in our case the nanowire morphology is retained after cycling while the other studies showed that $\mathrm{SnO}_{2}$ NW is destroyed while cycling. The SEM and TEM of a typical titania-coated $\mathrm{SnO}_{2} \mathrm{NWs}$ after cycling for 100 cycles are shown in Figure 6.6b. It is clear that titania- or aluminacoated $\mathrm{SnO}_{2} \mathrm{NW}$ morphology is maintained after 100 charge-discharge cycles. This is due to the presence of titania or alumina layer, which acts as a mechanical protective 
shield against pulverization during volume expansion, while still allowing lithium ions diffuse through to maintain cycling process. This further explains the high capacity retention at very high current density of $1500 \mathrm{mAg}^{-1}$. The presence of titania layer also helps to scavenge HF produced by the reaction of the trace amounts of water and $\mathrm{LiPF}_{6}$ in the electrolyte ${ }^{144}$. However, our observation reveals that all samples experienced severe delamination after 30 cycles with slow fading after 17 cycles (Figure 6.7). The columbic efficiency is $87 \%$ for the $1^{\text {st }}$ cycle, increases to $97 \%$ at the $3^{\text {rd }}$ cycle and remains up to $17^{\text {th }}$ cycle, then drop down to $89 \%$ at $30^{\text {th }}$ cycle. The decreasing of columbic efficiency is attributed to slow delamination from $17^{\text {th }}$ cycle to 30 cycles and then rapid delamination from 30 cycles to 100 cycles. As shown in SEM images of electrodes after 100 cycles, the morphology is completely retained while showing complete delamination from the substrate. Hence, the binder or some other coating is necessary to avoid the delamination.

(a)

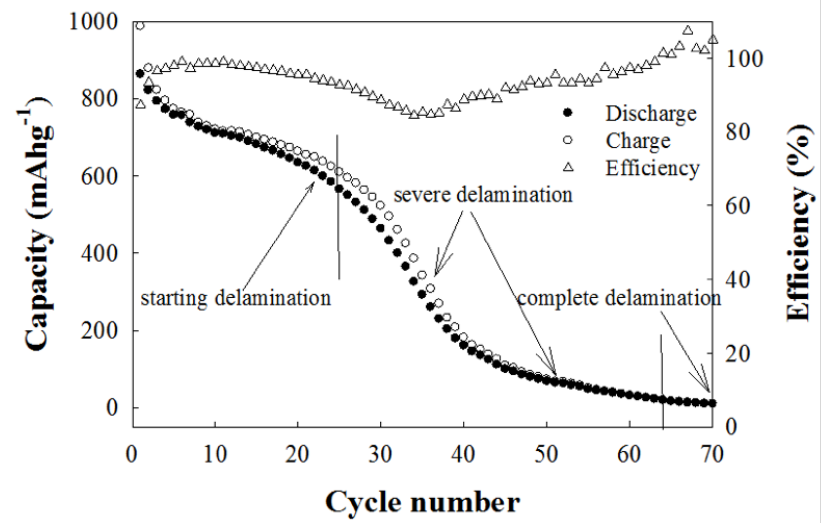

(b)

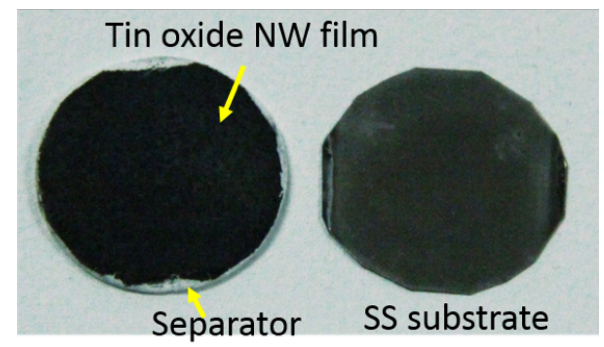

Figure 6.7: (a) Charge and discharge capacities vs. cycle number of titania-coated $\mathrm{SnO}_{2}$ NWs at current density of $1500 \mathrm{~mA} / \mathrm{g}$; (b) the photograph of delaminated electrode: the tin oxide film (black in color) was completely delaminated from stainless substrate and adhered onto the separator (white in color). 
In addition, Energy dispersive X-ray spectroscopy (EDS) data of titania- or alumina-coated $\mathrm{SnO}_{2} \mathrm{NW}$ electrodes after cycling revealed the presence of small tin cluster on the outer surface of $\mathrm{SnO}_{2} \mathrm{NW}$ (Figure 6.8). The observation of Sn nanocluster is much less when titania-coated $\mathrm{SnO}_{2} \mathrm{NWs}$ is cycled between 1.0 to $3.0 \mathrm{~V}$ allowing lithium intercalation into titania and not into $\mathrm{SnO}_{2}$ (Figure 6.9). The results suggest that the migration of tin out to the surface of coating layer happened when lithium ion intercalates into tin oxide. Similar results are obtained with thin alumina-coated $\mathrm{SnO}_{2}$ NW electrodes.

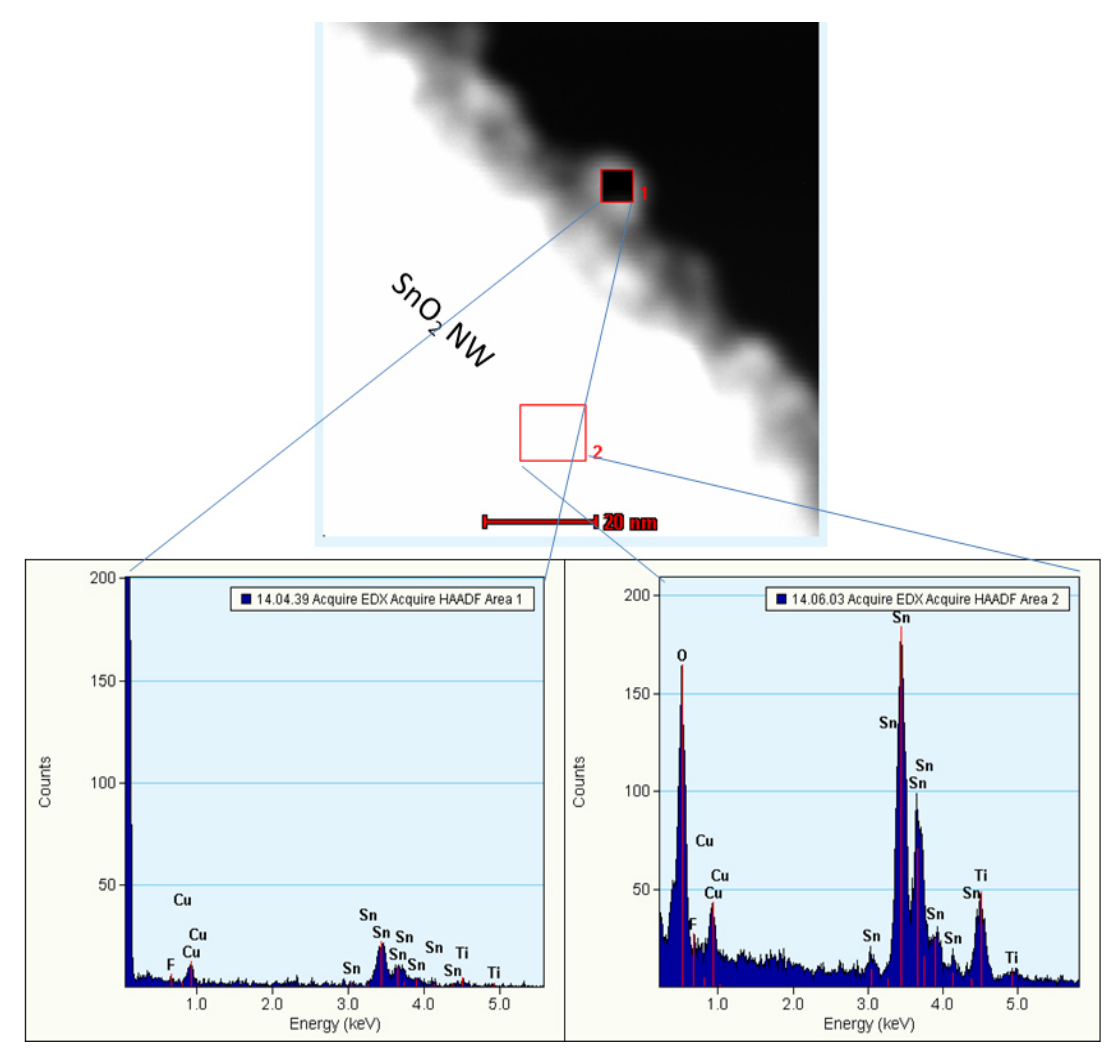

Figure 6.8: EDS data of $15 \mathrm{~nm}$ titania-coated $\mathrm{SnO}_{2} \mathrm{NWs}$ after cycling 


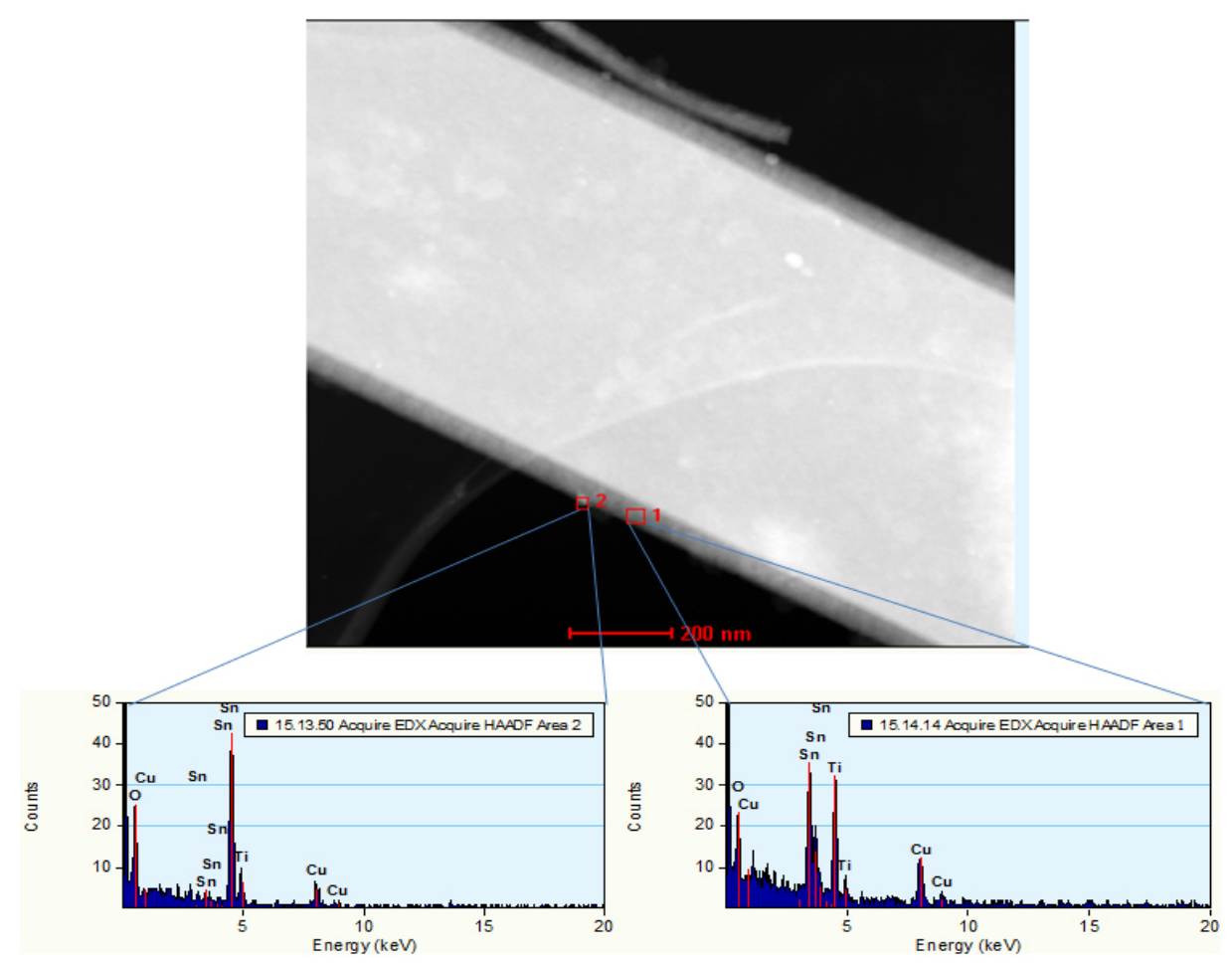

Figure 6.9: EDS data of titania-coated $\mathrm{SnO}_{2} \mathrm{NWs}$ after cycling at votage of $1.0-3.0 \mathrm{~V}$

The diffusion or migration of tin through the coatings is interesting and needs to be understood further. Much of the diffusion or migration seems to happen in the first few cycles. As these coatings (both titania and alumina) are rigid and thus develop stress during volume expansion experienced with lithium intercalation process. For a thin wall titania tube with external and internal nominal diameters of $\sim 75 \mathrm{~nm}$ and $\sim 65 \mathrm{~nm}$, Shokuhfar et al. ${ }^{145}$ has reported the maximum axial strain of 5\%. Assuming that the radial strain is the same as axial strain (it should be actually smaller ${ }^{146}$ ) the total volume expansion of a titania shell of $15 \mathrm{~nm}$ wall thickness, $300 \mathrm{~nm}$ inner diameter, can be calculated to be $115.7 \%$ with $5 \%$ radial and $5 \%$ axial elongation. This value is independent of wall thickness and inner diameter of titania nanotube. This confirms that the titania layer suppresses radial expansion and probably axial expansion as well since 
the volume expansion of tin oxide of $240 \%$ with $45 \%$ radial and $60 \%$ axial elongation ${ }^{147}$. Wang et al. ${ }^{148}$ has shown that $\mathrm{Li}_{\mathrm{x}} \mathrm{Sn}$ ball diffused out to the $\mathrm{SnO}_{2} \mathrm{NWs}$ surface upon $1^{\text {st }}$ charging cycle. In another study, Zhang et al. ${ }^{149}$ has reported that tin could precipitate out on the nanowire surface due to strain gradient and breaking of coating layer of $\mathrm{LiAlSiO}_{\mathrm{x}}$. In our study, tin nanoclusters come out on the surface of titania or alumina coating layer even when there are no apparent cracks in the coating layer when examined in TEM. Tin nanocluster seems to diffuse through titania layer without breaking it. Atomic scale tin could be produced during the reduction of tin oxide to tin or delithiation of $\mathrm{Li}_{\mathrm{x}} \mathrm{Sn}$ alloy. Tin atoms diffuse out on the surface to reduce stresses, tin crystal nucleate and coalesce to form tin nanocluster on the nanowire surface (Figure 6.10). This phenomenon has been widely observed for tin whisker growth in many other reports ${ }^{149-150}$. In contrast with a recent report on carbon coating on $\mathrm{SnO}_{2} \mathrm{NWs}^{129}$, the similar volume confinement has been seen with carbon coating on $\mathrm{SnO}_{2} \mathrm{NWs}$ but no tin nanocluster on the nanowire surface was observed. This may be due to the different properties of coatings such as electronic conductivity, tin and lithium diffusion rates. During cycling, the void accumulated inside the nanowire due to de-intercalation lead to the formation of hollow nanowire structure of $\mathrm{SnO}_{2}$ (Figure 6.11). Another explanation for hollow structure of $\mathrm{SnO}_{2}$ after cycling is due to the difference in the diffusion coefficients of lithium ion and tin atoms through coating layers of titania or alumina, and $\mathrm{Li}_{2} \mathrm{O}+\mathrm{Li}_{\mathrm{x}} \mathrm{Sn}+\mathrm{Sn}$ matrix. The Kirkendall effect is generally used to describe the formation of hollow structures which involves the diffusion of two species with different diffusion rates. The outward diffusion of tin may be faster than the inward diffusion of lithium ion. Thus, voids may be formed due to the transport of tin to the outer surface of the protective coatings. With time these 
voids, diffuses toward the center to form hollow core as seen in Figure 6.11, resulting in a Kirkendall effect ${ }^{151}$.

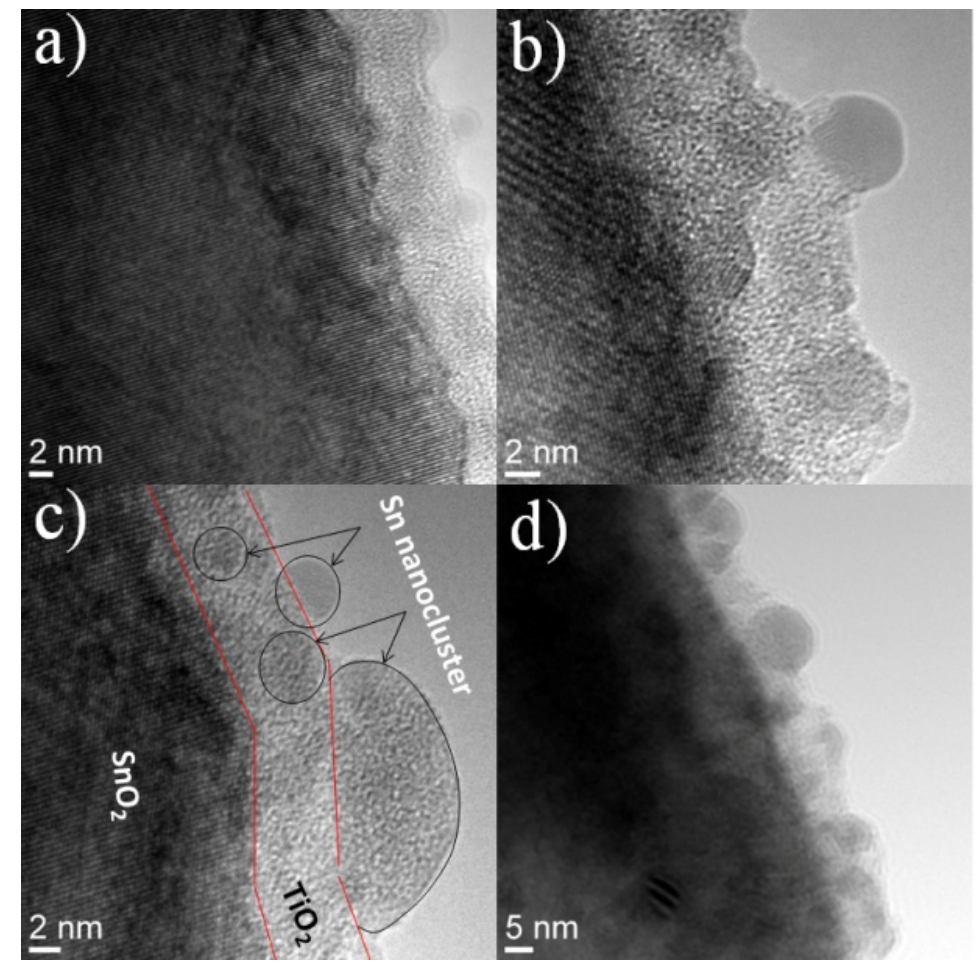

Figure 6.10: HR-TEM images of tin nanoclusters evolution on the surface of titania coating layer: tin started squeezing out a) and b); coalescing to form tin nanocluster c); tin nanoclusters on the nanowire surface d) 


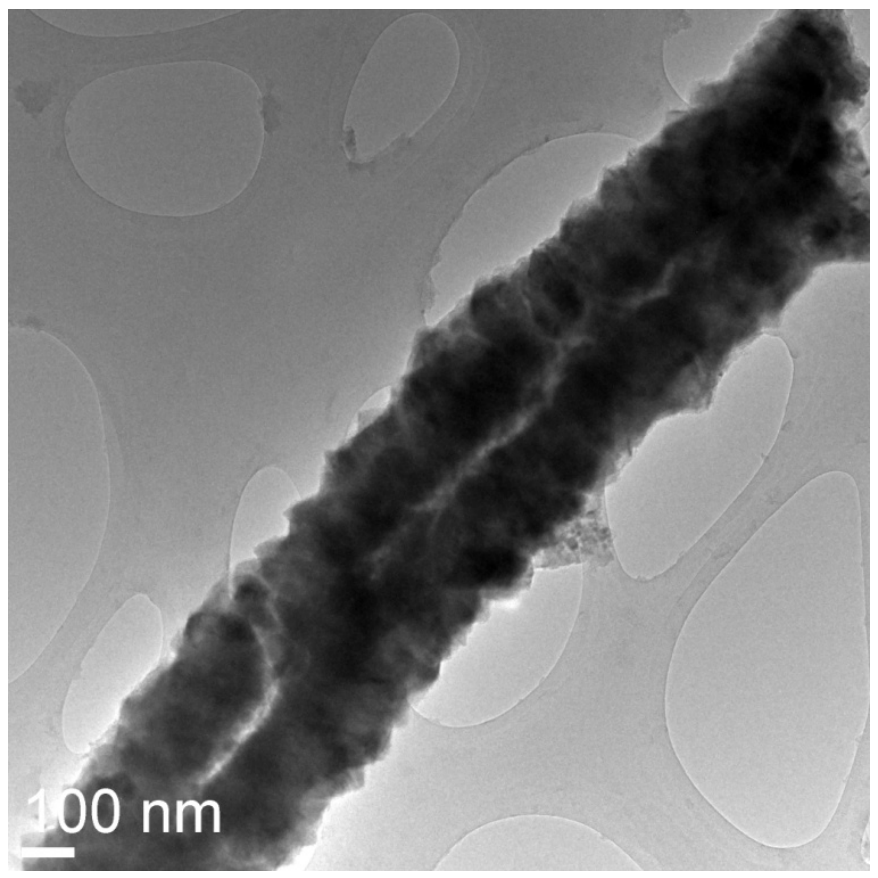

Figure 6.11: TEM image of titania-coated $\mathrm{SnO}_{2} \mathrm{NWs}$ after cycling at showing nanowire morphology with hollow structure

The presence of tin nanoclusters on the outer surface of $\mathrm{SnO}_{2} \mathrm{NWs}$ promotes the electronic conductivity of the electrodes which also explains the high capacity retention at very high current density ${ }^{137}$. The tin nanocluster formation during $1^{\text {st }}$ cylce (Figure 6.12) also trigger SEI formation from the $2^{\text {nd }}$ cycle and remain up to $10^{\text {th }}$ cycle as shown in Figure 2a. This is consistent with what is observed in cyclic voltammetry results shown in Figure 6.2. Figure 6.13 shows the SEM image of $5 \mathrm{~nm}$ titania, $1 \mathrm{~nm}$ aluminacoated $\mathrm{SnO}_{2} \mathrm{NW}$ after cycling for 52 cycles. Interestingly, the NW morphology is still maintained, wire-like morphology held by $\mathrm{Li}_{2} \mathrm{O}$ matrix with $\mathrm{Sn}$ particles, but the coating seems to dissolve in ethanol during sample preparation. These results suggest that even 1 nm thin coatings could protect the $\mathrm{SnO}_{2}$ NW morphology. The Young's and bulk modulus of titania and alumina (Table 6.2) suggest that alumina coating will be more rigid exerting more stress during lithium intercalation. The TEM-based EDS analysis of 1 
nm thick alumina-coated $\mathrm{SnO}_{2} \mathrm{NWs}$ suggests the presence of $\mathrm{Sn}$ particles and some carbon, fluorine, phosphor materials which is believed to come from the decomposition of electrolyte (Figure 6.14). These materials are reported to be a stable SEI layer which help to increase the rate capacity ${ }^{142}$.
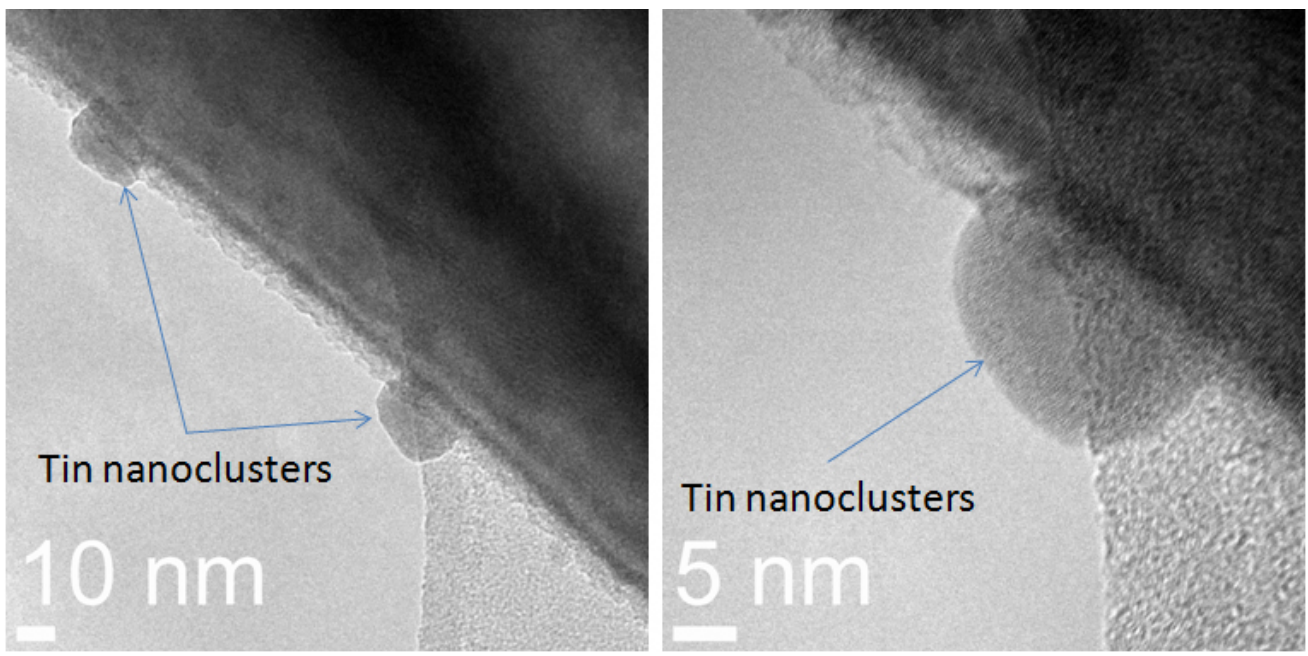

Figure 6.12: HR-TEM of titania-coated $\mathrm{SnO}_{2} \mathrm{NWs}$ after $1^{\text {st }}$ cycle showing presence of tin nanoclusters on nanowire surface

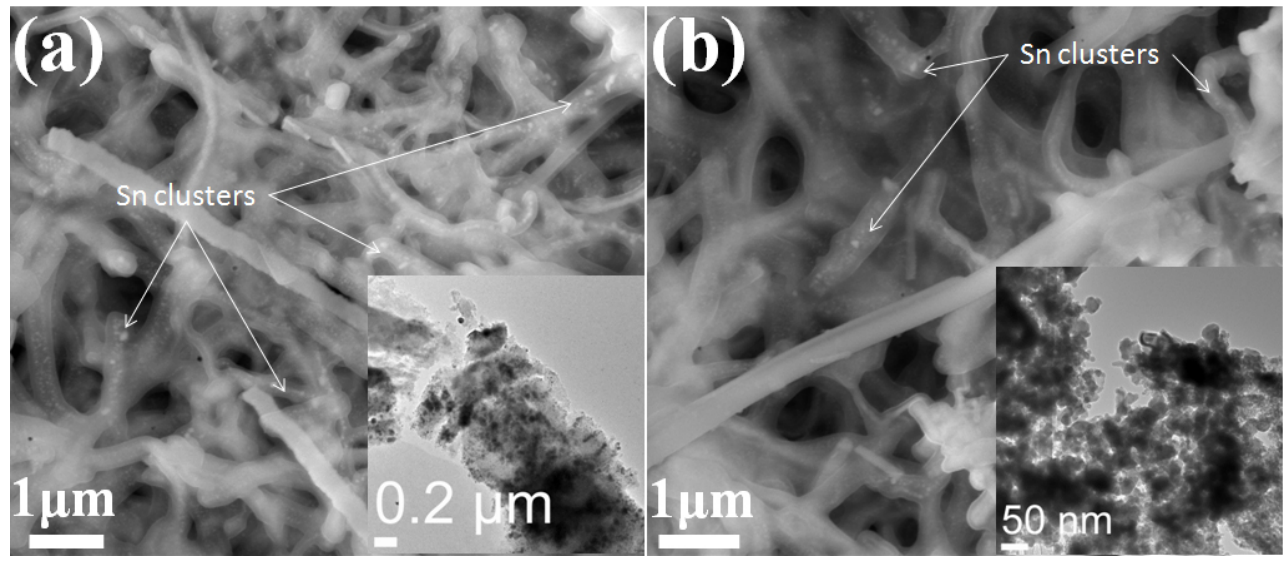


Figure 6.13: SEM, TEM (inset) images of thin layer of titania-(a), alumina-(b) coated $\mathrm{SnO}_{2}$ NWs after cycling. The white spot as Sn clusters are presence in either titania- or alumina-coated $\mathrm{SnO}_{2} \mathrm{NWs}$

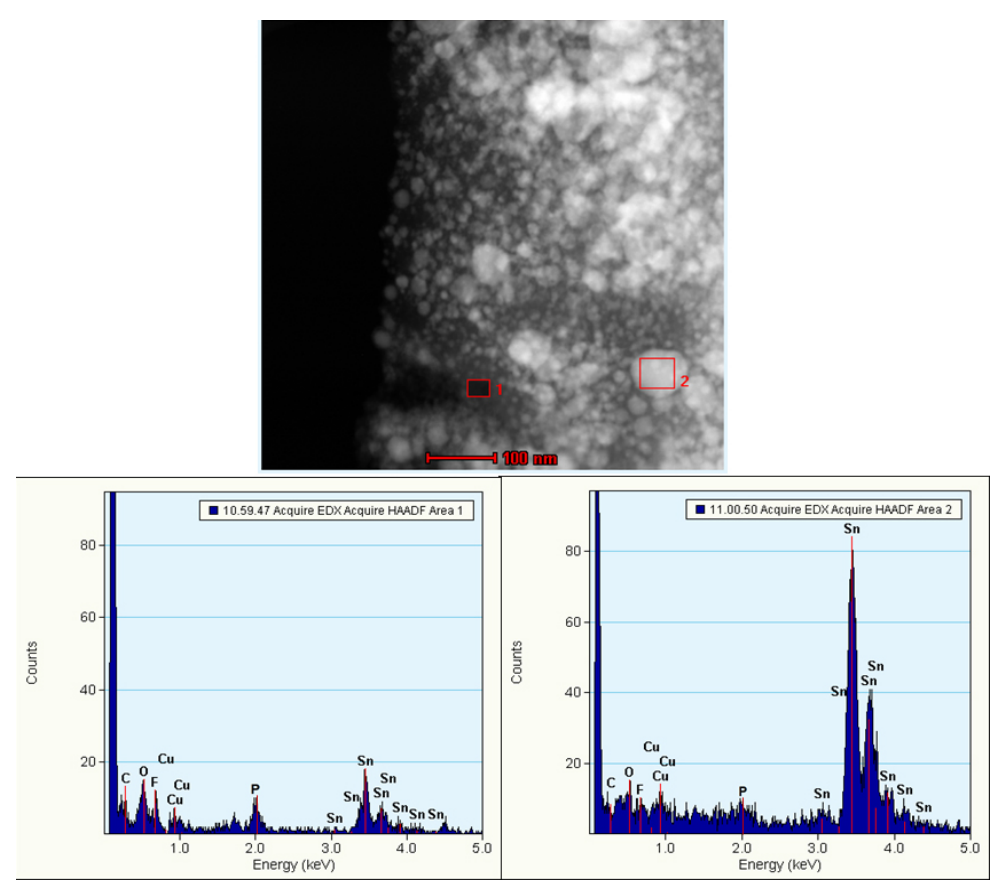

Figure 6.14: EDS data of $5 \mathrm{~nm}$ titania-coated $\mathrm{SnO}_{2} \mathrm{NWs}$ after cycling

Table 6.2: Young's and bulk modulus of alumina and titania

\begin{tabular}{|c|c|c|c|}
\hline & $\mathbf{E}, \mathbf{G P a}$ & G, GPa & Structure \\
\hline \multirow{2}{*}{ Titania } & $36-43$ & & $10-18 \mathrm{~nm}$ wall thickness, $35-70 \mathrm{~nm}$ diameter, nanotube \\
\hline & 23 & & $10 \mathrm{~nm}$ wall thickness, $65 \mathrm{~nm}$ diameter, nanotube \\
\hline
\end{tabular}


44

151

146

140
$30 \mathrm{~nm}$ wall thickness, $80 \mathrm{~nm}$ diameter, nanotube

$200 \mathrm{~nm}$ thickness, anatase film

$280 \mathrm{~nm}$ thickness film

168-182

50-300 nm thickness film

Alumina

235

\subsection{Conclusions}

In summary, titania and alumina coatings have been investigated on $\mathrm{SnO}_{2} \mathrm{NWs}$ without using any binders. Ultra-thin layers as thin as $1 \mathrm{~nm}$ stabilized the onedimmesional morphology of $\mathrm{SnO}_{2}$ nanowires and allowed for high capacity retention after 30 cycles at high rates (over $767 \mathrm{mAhg}^{-1}$ at $1 \mathrm{C}$ and $664 \mathrm{mAhg}^{-1}$ at 2C). No initial capacity loss due to SEI formation was found which increased the reversible capacity retention. Interestingly, both titania- and alumina-coated tin oxide nanowire arrays exhibited tin migration through the coatings to form tin nanoclusters. The compressive stress build-up during lithium intercalation and the enhanced diffusion of tin during lithium de-intercalation allowed for migration of tin to outside of coatings. The knowledge on the stability of ultra-thin coatings during lithium intercalation/deintercalation is important for many materials systems. The results obtained with tin should be applicable to other high capacity materials such as silicon. 


\section{CHAPTER VII}

\section{CONCLUSIONS}

\subsection{The fluidized bed reactor}

- The scalability of nanowire production using the downstream atmospheric microwave reactor has been shown to be limited due to short residence time and plasma instability issues. Even with limited scale of 100 grams batch runs per day, the production studies have shown that the yield can be improved to $95 \%$ using various reactor modifications on powder feeding, collection and cooling of powder plume.

- An upward conical fluidized bed reactor employing atmospheric microwave and hydrocarbon flames is designed and operated for 10 kilograms per day scale production of metal oxide nanowires.

- The fluidization in a fluidized bed reactor has been shown to increase the residence time which seemed to help with conversion.

- There is also a cyclone and a filter housing which enables the efficient powder collection and allows continuous production for long period of time 


\subsection{Large scale production of zinc oxide nanowires using fluidized bed reactor}

- The single crystalline nanowires produced using microwave plasma are about 20$80 \mathrm{~nm}$ in diameter, about 1 micron in length, aspect ratio of $6-20$. The single crystalline nanowires produced using hydrocarbon are larger in diameter of about $80-150 \mathrm{~nm}$, about the same length of 1 micron, aspect ratio of $2-10$.

- The production rate as high as 1.2 kilograms per hour is obtained using hydrocarbon flame. The yield is about $30 \%$ using microwave plasma flame and is about $90 \%$ using hydrocarbon.

- The nanowire morphology can be controlled by varying the operating parameters such as flame source, gas flow rate, zinc feeding rate, etc.

- The yield can be increased by increasing hydrocarbon flow rate and flame volume. The production capacity can be increased by increasing the feeding rate, hydrocarbon flow rate, and flame volume.

\subsection{Solvo-plasma production of metal oxide nanowires}

- Showed that solvo-plasma oxidation concept works with low-melting point metal oxide systems as well. In particular, tin oxide nanowires were obtained using plasma oxidation of either tin or tin oxide mixed with alkali compounds. The reaction time scales of a few seconds to a minute making these techniques as highly scalable.

- A fast nanowire growth rate of $1 \mu \mathrm{m} / \mathrm{min}$ was observed which is 25 time higher than that with thermal oxidation techniques. The use of tin mono-oxide as precursor has been shown to have the growth rate up to $40 \mu \mathrm{m} / \mathrm{min}$ for solvoplasma oxidation technique 
- The presence of intermediate phase of potassium stannate has been observed which could be responsible for the observed one-dimensional growth. Further acid wash and annealing of potassium stannate allowed for formation of porous tin oxide nanowires. The observation of intermediate compound with layered structure could provide a clue to explain one-dimensional growth observed with other materials systems with solvo-plasma oxidation concept.

- The solvo-plasma technique seems to be a generic scheme for synthesizing most of metal oxides including titanium, cobalt, manganese, tungsten, zinc and tin oxide.

- Solvo-plasma is a fast, scalable method which is an ideal replacement for several liquid phase techniques such as hydrothermal, precipitation, electrochemical syntheses. A simple lab-scale roll-to-roll setup can produce up to 300 grams per hour.

\subsection{Applications of nanowires as catalyst supports and lithium ion batteries}

- Zinc oxide nanowires were used as catalytic adsorbent supports for nickel clusters. Extrudates made using zinc oxide nanowires decorated with 6-15 wt\% nickel clusters and $\gamma$-alumina as binder were shown to remove sulfur content in diesel from 30-200 ppm to less than $1 \mathrm{ppm}$. The steady state activity of the catalyst was achieved within the first few hours and remained constant for over 100 hour tests.

- Ultra-thin layers of titania and alumina as thin as $1 \mathrm{~nm}$ stabilized the onedimmesional morphology of $\mathrm{SnO}_{2}$ nanowires and allowed for high capacity retention after 30 cycles at high rates (over $767 \mathrm{mAhg}^{-1}$ at $1 \mathrm{C}$ and $664 \mathrm{mAhg}^{-1}$ at 
2C). No initial capacity loss due to SEI formation was found which increased the reversible capacity retention.

- Both titania- and alumina-coated tin oxide nanowire arrays exhibited tin migration through the coatings to form tin nanoclusters. The compressive stress build-up during lithium intercalation and the enhanced diffusion of tin during lithium deintercalation allowed for migration of tin to outside of coatings. The results obtained with tin should be applicable to other high capacity materials such as silicon.

- Porous tin oxide nanowires (with surface area of $36 \mathrm{~m}^{2} / \mathrm{g}$ ) showed a high reversible capacity of $848 \mathrm{mAh} \mathrm{g}^{-1}$ after 55 cycles at a current density of 100 $\mathrm{mA} / \mathrm{g}$. 


\section{CHAPTER VIII}

\section{RECOMMENDATIONS FOR FUTURE WORK}

In this dissertation, methods and reactors for producing metal oxide nanowires of zinc and tin are described in addition to brief discussions of applications of zinc oxide and tin oxide nanowires as catalyst support for hydro-desulfurization and anodes for lithium ion battery, respectively. Following are the recommendations for future works:

- Incorporation of plasma flame in the fluidized bed for controlling the nanowire morphology and yield can be achieved. The plasma flame would provide highly active species such as electrons, ions, neutrals in addition to main hydrocarbon flame sources. The previous experiments show that higher aspect ratio, narrower in diameter and longer in length of nanowires were produced using plasma flame. Therefore, small amount of these active species in combination with hydrocarbon would result in desired aspect ratio, diameter, and length of nanowires.

- The plasma flame should be introduced nearby the bottom inlet at an angle to avoid the heating to quartz. A high flow should be used for the plasma to avoid the backstreams to applicator. Oxygen plasma is highly recommended to increase the active oxygen species necessary for promoting nanowire growth and 
increasing yield.

- The fluidized bed reactor can be extended to other low melting point metals such as tin, aluminum, indium, and titania. The results can be either nanowires or nanoparticles but both will be useful for different applications.

- Experiments with mix-precursors should be a good try for support-free nanowire growth using solvo-plasma techniques in the fluidized bed reactor. For example, mixture of tin oxide particles and potassium oxides can be fed into fluidized bed reactor to form stannate nanowires. In some cases, mixed metal oxide particles can be produced for many exciting applications.

- In-situ TEM experiment for the growth of metal oxide nanowires using their corresponding oxide particles could give more valuable mechanistic insights of nanowire formation from particles via solvo plasma. Fundamental studies of this scheme would provide valuable information for designing nanomaterials of many other material systems using solvo-plasma technique. 


\section{REFERENCES}

1. Iijima, S., Helical microtubules of graphitic carbon. Nature 1991, 354 (6348), 5658.

2. Jr., J. F. S., The National Nanotechnology Initiative: Overview, Reauthorization, and Appropriations Issues. 2014, 71.

3. Taberna, P. L.; Mitra, S.; Poizot, P.; Simon, P.; Tarascon, J. M., High rate capabilities Fe3O4-based $\mathrm{Cu}$ nano-architectured electrodes for lithium-ion battery applications. Nat Mater 2006, 5 (7), 567-573.

4. Appell, D., Nanotechnology: Wired for success. Nature 2002, 419 (6907), 553555.

5. (a) Kolmakov, A.; Potluri, S.; Barinov, A.; Menteş, T. O.; Gregoratti, L.; Niño, M. A.; Locatelli, A.; Kiskinova, M., Spectromicroscopy for Addressing the Surface and Electron Transport Properties of Individual 1-D Nanostructures and Their Networks. ACS Nano 2008, 2 (10), 1993-2000; (b) Meduri, P.; Pendyala, C.; Kumar, V.; Sumanasekera, G. U.; Sunkara, M. K., Hybrid Tin Oxide Nanowires as Stable and High Capacity Anodes for Li-Ion Batteries. Nano Letters 2009, 9 (2), 612-616.

6. Behl, M.; Yeom, J.; Lineberry, Q.; Jain, P. K.; Shannon, M. A., A regenerable oxide-based H2S adsorbent with nanofibrous morphology. Nat Nano 2012, 7 (12), 810815. 
7. Gubbala, S.; Chakrapani, V.; Kumar, V.; Sunkara, M. K., Band-Edge Engineered Hybrid Structures for Dye-Sensitized Solar Cells Based on SnO2 Nanowires. Advanced Functional Materials 2008, 18 (16), 2411-2418.

8. Peng, Z.; Ping, Z.; Ming, H.; Shuang-Yun, M.; Wen-Jun, Y., Synthesis and roomtemperature NO 2 gas sensing properties of a WO 3 nanowires/porous silicon hybrid structure. Chinese Physics B 2014, 23 (5), 058103.

9. Vivekchand, S. R. C.; Kam, K. C.; Gundiah, G.; Govindaraj, A.; Cheetham, A. K.; Rao, C. N. R., Electrical properties of inorganic nanowire-polymer composites. Journal of Materials Chemistry 2005, 15 (46), 4922-4927.

10. Sunkara, M. K.; Pendyala, C.; Cummins, D.; Meduri, P.; Jasinski, J.; Kumar, V.; Russell, H. B.; Clark, E. L.; Kim, J. H., Inorganic nanowires: a perspective about their role in energy conversion and storage applications. Journal of Physics D: Applied Physics 2011, 44 (17), 174032.

11. Christopher, P.; Linic, S., Engineering selectivity in heterogeneous catalysis: Ag nanowires as selective ethylene epoxidation catalysts. Journal of the American Chemical Society 2008, 130 (34), 11264-11265.

12. Petzold, F. G.; Jasinski, J.; Clark, E. L.; Kim, J. H.; Absher, J.; Toufar, H.; Sunkara, M. K., Nickel supported on zinc oxide nanowires as advanced hydrodesulfurization catalysts. Catalysis Today 2012, 198 (1), 219-227.

13. Gupta, M.; He, J.; Nguyen, T.; Petzold, F.; Fonseca, D.; Jasinski, J. B.; Sunkara, M. K., "Nanowire catalysts for ultra-deep hydro-desulfurization and aromatic hydrogenation". Applied Catalysis B: Environmental 2016, 180, 246-254. 
14. G.L. Henriksen, K. A., J. Liu and P.A. Nelson, Materials cost evaluation report for high power li-ion HEV batteries. 2002.

15. (a) Kumar, V.; Kim, J. H.; Jasinski, J. B.; Clark, E. L.; Sunkara, M. K., AlkaliAssisted, Atmospheric Plasma Production of Titania Nanowire Powders and Arrays. Crystal Growth \& Design 2011, 11 (7), 2913-2919; (b) Kumar, V.; Kim, J. H.; Pendyala, C.; Chernomordik, B.; Sunkara, M. K., Gas-Phase, Bulk Production of Metal Oxide Nanowires and Nanoparticles Using a Microwave Plasma Jet Reactor. Journal of Physical Chemistry C 2008, 112 (46), 17750-17754.

16. Kumar, V.; Kim, J. H.; Pendyala, C.; Chernomordik, B.; Sunkara, M. K., GasPhase, Bulk Production of Metal Oxide Nanowires and Nanoparticles Using a Microwave Plasma Jet Reactor. The Journal of Physical Chemistry C 2008, 112 (46), 17750-17754.

17. Pratsinis, S. E., Flame aerosol synthesis of ceramic powders. Progress in Energy and Combustion Science 1998, 24 (3), 197-219.

18. Wegner, K.; Schimmöller, B.; Thiebaut, B.; Fernandez, C.; Rao, T. N., Pilot Plants for Industrial Nanoparticle Production by Flame Spray Pyrolysis. KONA Powder and Particle Journal 2011, 29, 251-265.

19. Wagner, R. S.; Ellis, W. C., VAPOR-LIQUID-SOLID MECHANISM OF SINGLE CRYSTAL GROWTH. Applied Physics Letters 1964, 4 (5), 89-90.

20. Choi, H.-J., Vapor-Liquid-Solid Growth of Semiconductor Nanowires. In Semiconductor Nanostructures for Optoelectronic Devices: Processing, Characterization and Applications, Yi, G.-C., Ed. Springer Berlin Heidelberg: Berlin, Heidelberg, 2012; pp 1-36. 
21. Terasako, T.; Hambali, N. A.; Jayah, N. A.; Wakisaka, T.; Hashim, A. M.; Yagi, M., Shape controlled growth of $\mathrm{ZnO}$ nanorods and fabrication of $\mathrm{ZnO} / \mathrm{CuO}$ heterojunctions by chemical bath deposition using zinc nitrate hexahydrate and copper (III) nitrate trihydrate. Thin Solid Films 2015, 596, 201-208.

22. Cheng, J. J.; Nicaise, S. M.; Berggren, K. K.; Gradečak, S., Dimensional Tailoring of Hydrothermally Grown Zinc Oxide Nanowire Arrays. Nano Letters 2016, 16 (1), 753-759.

23. Guo, J.; Zhang, J.; Zhu, M.; Ju, D.; Xu, H.; Cao, B., High-performance gas sensor based on $\mathrm{ZnO}$ nanowires functionalized by $\mathrm{Au}$ nanoparticles. Sensors and Actuators B: Chemical 2014, 199, 339-345.

24. Strelchuk, V. V.; Nikolenko, A. S.; Kolomys, O. F.; Rarata, S. V.; Avramenko, K. A.; Lytvyn, P. M.; Tronc, P.; Chey, C. O.; Nur, O.; Willander, M., Optical and structural properties of $\mathrm{Mn}$-doped $\mathrm{ZnO}$ nanorods grown by aqueous chemical growth for spintronic applications. Thin Solid Films 2016, 601, 22-27.

25. Pal, U.; Pal, M.; Zeferino, R. S., Gram-scale synthesis of highly crystalline, 0-D and 1-D SnO2 nanostructures through surfactant-free hydrothermal process. Journal of Nanoparticle Research 2012, 14 (7), 1-10.

26. Wang, Z.; Hu, M.; Wei, Y.; Liu, J.; Qin, Y., Low-temperature NO2-sensing properties and morphology-controllable solvothermal synthesis of tungsten oxide nanosheets/nanorods. Applied Surface Science 2016, 362, 525-531.

27. Wu, W.-Q.; Lei, B.-X.; Rao, H.-S.; Xu, Y.-F.; Wang, Y.-F.; Su, C.-Y.; Kuang, D.B., Hydrothermal Fabrication of Hierarchically Anatase TiO2 Nanowire arrays on FTO Glass for Dye-sensitized Solar Cells. Scientific Reports 2013, 3, 1352. 
28. Cheng, G.; Yu, L.; Lan, B.; Sun, M.; Lin, T.; Fu, Z.; Su, X.; Qiu, M.; Guo, C.; $\mathrm{Xu}, \mathrm{B}$., Controlled synthesis of $\alpha-\mathrm{MnO} 2$ nanowires and their catalytic performance for toluene combustion. Materials Research Bulletin 2016, 75, 17-24.

29. Dou, Z.; Cao, C.; Chen, Y.; Song, W., Fabrication of porous Co3O4 nanowires with high $\mathrm{CO}$ sensing performance at a low operating temperature. Chemical Communications 2014, 50 (94), 14889-14891.

30. Liu, L.; Hong, K.; Hu, T.; Xu, M., Synthesis of aligned copper oxide nanorod arrays by a seed mediated hydrothermal method. Journal of Alloys and Compounds 2012, $511(1), 195-197$.

31. Navarro, J. R. G.; Mayence, A.; Andrade, J.; Lerouge, F.; Chaput, F.; Oleynikov, P.; Bergström, L.; Parola, S.; Pawlicka, A., WO3 Nanorods Created by Self-Assembly of Highly Crystalline Nanowires under Hydrothermal Conditions. Langmuir 2014, 30 (34), 10487-10492.

32. Zheng, M. J.; Zhang, L. D.; Li, G. H.; Shen, W. Z., Fabrication and optical properties of large-scale uniform zinc oxide nanowire arrays by one-step electrochemical deposition technique. Chemical Physics Letters 2002, 363 (1-2), 123-128.

33. Lai, M.; Gonzalez Martinez, J. A.; Gratzel, M.; Riley, D. J., Preparation of tin dioxide nanotubes via electrosynthesis in a template. Journal of Materials Chemistry 2006, 16 (27), 2843-2845.

34. Wu, G. S.; Xie, T.; Yuan, X. Y.; Li, Y.; Yang, L.; Xiao, Y. H.; Zhang, L. D., Controlled synthesis of $\mathrm{ZnO}$ nanowires or nanotubes via sol-gel template process. Solid State Communications 2005, 134 (7), 485-489. 
35. (a) Hsieh, C.-T.; Yang, S.-Y.; Gu, J.-L.; Jiang, Y.-R., Influence of growth parameters on texture of $\mathrm{ZnO}$ nanorods by using electrochemical deposition at low temperatures. Solid State Ionics 2012, 209-210, 43-50; (b) Tamvakos, D.; Lepadatu, S.; Antohe, V.-A.; Tamvakos, A.; Weaver, P. M.; Piraux, L.; Cain, M. G.; Pullini, D., Piezoelectric properties of template-free electrochemically grown $\mathrm{ZnO}$ nanorod arrays. Applied Surface Science 2015, 356, 1214-1220.

36. Katwal, G.; Paulose, M.; Rusakova, I. A.; Martinez, J. E.; Varghese, O. K., Rapid Growth of Zinc Oxide Nanotube-Nanowire Hybrid Architectures and Their Use in Breast Cancer-Related Volatile Organics Detection. Nano Letters 2016, 16 (5), 3014-3021.

37. Cho, S.; Jung, S.-H.; Lee, K.-H., Morphology-Controlled Growth of $\mathrm{ZnO}$ Nanostructures Using Microwave Irradiation: from Basic to Complex Structures. The Journal of Physical Chemistry C 2008, 112 (33), 12769-12776.

38. Hamedani, N. F.; Mahjoub, A. R.; Khodadadi, A. A.; Mortazavi, Y., Microwave assisted fast synthesis of various $\mathrm{ZnO}$ morphologies for selective detection of $\mathrm{CO}, \mathrm{CH} 4$ and ethanol. Sensors and Actuators B: Chemical 2011, 156 (2), 737-742.

39. Husnu Emrah, U.; Pritesh, H.; Nalin, R.; Sharvari, D.; William, I. M.; Gehan, A. J. A., Rapid synthesis of aligned zinc oxide nanowires. Nanotechnology 2008, 19 (25), 255608.

40. Krishna, M.; Komarneni, S., Conventional- vs microwave-hydrothermal synthesis of tin oxide, $\mathrm{SnO} 2$ nanoparticles. Ceramics International 2009, 35 (8), 3375-3379.

41. Wang, H. E.; Xi, L. J.; Ma, R. G.; Lu, Z. G.; Chung, C. Y.; Bello, I.; Zapien, J. A., Microwave-assisted hydrothermal synthesis of porous $\mathrm{SnO} 2$ nanotubes and their lithium ion storage properties. Journal of Solid State Chemistry 2012, 190, 104-110. 
42. Sharma, S.; Sunkara, M. K., Direct Synthesis of Gallium Oxide Tubes, Nanowires, and Nanopaintbrushes. Journal of the American Chemical Society 2002, 124 (41), 12288-12293.

43. Sunkara, M. K.; Sharma, S.; Miranda, R.; Lian, G.; Dickey, E. C., Bulk synthesis of silicon nanowires using a low-temperature vapor-liquid-solid method. Applied Physics Letters 2001, 79 (10), 1546-1548.

44. Roso, S.; Güell, F.; Martínez-Alanis, P. R.; Urakawa, A.; Llobet, E., Synthesis of $\mathrm{ZnO}$ nanowires and impacts of their orientation and defects on their gas sensing properties. Sensors and Actuators B: Chemical 2016, 230, 109-114.

45. Yuan, L.; Wang, C.; Cai, R.; Wang, Y.; Zhou, G., Temperature-dependent growth mechanism and microstructure of $\mathrm{ZnO}$ nanostructures grown from the thermal oxidation of zinc. Journal of Crystal Growth 2014, 390, 101-108.

46. Fung, M. K.; Wong, K. K.; Chen, X. Y.; Chan, Y. F.; Ng, A. M. C.; Djurišić, A. B.; Chan, W. K., Indium oxide, tin oxide and indium tin oxide nanostructure growth by vapor deposition. Current Applied Physics 2012, 12 (3), 697-706.

47. Gubbala, S.; Russell, H. B.; Shah, H.; Deb, B.; Jasinski, J.; Rypkema, H.; Sunkara, M. K., Surface properties of SnO2nanowires for enhanced performance with dye-sensitized solar cells. Energy \& Environmental Science 2009, 2 (12), 1302-1309.

48. Thangala, J.; Vaddiraju, S.; Bogale, R.; Thurman, R.; Powers, T.; Deb, B.; Sunkara, M. K., Large-Scale, Hot-Filament-Assisted Synthesis of Tungsten Oxide and Related Transition Metal Oxide Nanowires. Small 2007, 3 (5), 890-896. 
49. Vaddiraju, S.; Chandrasekaran, H.; Sunkara, M. K., Vapor Phase Synthesis of Tungsten Nanowires. Journal of the American Chemical Society 2003, 125 (36), 1079210793.

50. Sunkara, S.; Vendra, V. K.; Jasinski, J. B.; Deutsch, T.; Andriotis, A. N.; Rajan, K.; Menon, M.; Sunkara, M., New Visible Light Absorbing Materials for Solar Fuels, Ga(Sbx)N1-x. Advanced Materials 2014, 26 (18), 2878-2882.

51. Dikovska, A. O.; Atanasova, G. B.; Avdeev, G. V.; Nedyalkov, N. N., Synthesis and characterization of $\mathrm{ZnO}$ nanostructures on noble-metal coated substrates. Applied Surface Science 2016, 374, 65-70.

52. Morales, A. M.; Lieber, C. M., A Laser Ablation Method for the Synthesis of Crystalline Semiconductor Nanowires. Science 1998, 279 (5348), 208-211.

53. Masaya, S.; Anthony, B. M., Thermal plasmas for nanofabrication. Journal of Physics D: Applied Physics 2011, 44 (17), 174025.

54. Cvelbar, U., Towards large-scale plasma-assisted synthesis of nanowires. Journal of Physics D: Applied Physics 2011, 44 (17), 174014.

55. Liu, X.; Wu, X.; Cao, H.; Chang, R. P. H., Growth mechanism and properties of $\mathrm{ZnO}$ nanorods synthesized by plasma-enhanced chemical vapor deposition. Journal of Applied Physics 2004, 95 (6), 3141-3147.

56. Maria, L. C.; Jasinski, J. B.; Sunkara, M. K., Low temperature synthesis of silicon nanowire arrays. Materials Research Express 2014, 1 (4), 045006.

57. (a) Ostrikov, K.; Levchenko, I.; Cvelbar, U.; Sunkara, M.; Mozetic, M., From nucleation to nanowires: a single-step process in reactive plasmas. Nanoscale 2010, 2 (10), 2012-2027; (b) Cvelbar, U.; Chen, Z.; Sunkara, M. K.; Mozetič, M., Spontaneous 
Growth of Superstructure $\alpha-\mathrm{Fe} 2 \mathrm{O} 3$ Nanowire and Nanobelt Arrays in Reactive Oxygen Plasma. Small 2008, 4 (10), 1610-1614.

58. Ko, T. S.; Yang, S.; Hsu, H. C.; Chu, C. P.; Lin, H. F.; Liao, S. C.; Lu, T. C.; Kuo, H. C.; Hsieh, W. F.; Wang, S. C., ZnO nanopowders fabricated by de thermal plasma synthesis. Materials Science and Engineering: B 2006, 134 (1), 54-58.

59. Peng, H.; Fangli, Y.; Liuyang, B.; Jinlin, L.; Yunfa, C., Plasma Synthesis of Large Quantities of Zinc Oxide Nanorods. The Journal of Physical Chemistry C 2007, 111 (1), 194-200.

60. Wooldridge, M. S.; Danczyk, S. A.; Wu, J., Demonstration of gas-phase combustion synthesis of nanosized particles using a hybrid burner. Nanostructured Materials 1999, 11 (7), 955-964.

61. Strobel, R.; Alfons, A.; Pratsinis, S. E., Aerosol flame synthesis of catalysts. Advanced Powder Technology 2006, 17 (5), 457-480.

62. Koirala, R.; Pratsinis, S. E.; Baiker, A., Synthesis of catalytic materials in flames: opportunities and challenges. Chemical Society Reviews 2016, 45 (11), 3053-3068.

63. Mädler, L., Liquid-fed Aerosol Reactors for One-step Synthesis of Nanostructured Particles. KONA Powder and Particle Journal 2004, 22, 107-120.

64. Hembram, K.; Sivaprakasam, D.; Rao, T. N.; Wegner, K., Large-scale manufacture of $\mathrm{ZnO}$ nanorods by flame spray pyrolysis. Journal of Nanoparticle Research 2013, 15 (2), 1-11.

65. Tani, T.; Mädler, L.; Pratsinis, S. E., Homogeneous ZnO Nanoparticles by Flame Spray Pyrolysis. Journal of Nanoparticle Research 2002, 4 (4), 337-343. 
66. Sahm, T.; Mädler, L.; Gurlo, A.; Barsan, N.; Pratsinis, S. E.; Weimar, U., Flame spray synthesis of tin dioxide nanoparticles for gas sensing. Sensors and Actuators B: Chemical 2004, $98(2-3), 148-153$.

67. Mädler, L.; Kammler, H. K.; Mueller, R.; Pratsinis, S. E., Controlled synthesis of nanostructured particles by flame spray pyrolysis. Journal of Aerosol Science 2002, 33 (2), 369-389.

68. Gröhn, A. J.; Pratsinis, S. E.; Sánchez-Ferrer, A.; Mezzenga, R.; Wegner, K., Scale-up of Nanoparticle Synthesis by Flame Spray Pyrolysis: The High-Temperature Particle Residence Time. Industrial \& Engineering Chemistry Research 2014, 53 (26), 10734-10742.

69. Height, M. J.; Howard, J. B.; Tester, J. W.; Vander Sande, J. B., Flame synthesis of single-walled carbon nanotubes. Carbon 2004, 42 (11), 2295-2307.

70. Height, M. J.; Mädler, L.; Pratsinis, S. E.; Krumeich, F., Nanorods of ZnO Made by Flame Spray Pyrolysis. Chemistry of Materials 2006, 18 (2), 572-578.

71. Vishwanath Gandikota, Y. X., Flame aerosol synthesis of freestanding ZnO nanorods. Advances in Nanoparticles 2014, 3, 9.

72. Kumar, V., Novel scalable, atmospheric plasma based manufacturing methods for metal oxide nanowires. PhD Dissertation, University of Louisville 2011.

73. Foggiato, J., Chemical Vapor Deposition of Silicon Dioxide Films, Handbook of Thin Film Deposition. 2001.

74. Guo, J.; Fan, X.; Richard, D.; Xue, S.; Jerzy, J.; Maher, B., Development of Nanopowder Synthesis Using Induction Plasma. Plasma Science and Technology 2010, $12(2), 188$. 
75. Eric, W. P.; Edward, M. L.; Kasey, J. R.; Venkatesh, N., Growth of ZnO nanowires catalyzed by size-dependent melting of Au nanoparticles. Nanotechnology 2009, 20 (40), 405603.

76. See, C. H.; Harris, A. T., A Review of Carbon Nanotube Synthesis via FluidizedBed Chemical Vapor Deposition. Industrial \& Engineering Chemistry Research 2007, 46 (4), 997-1012.

77. Sunkara, M. K.; Nguyen, T. Q.; Guhy, L. H.; Paxton, W. F., Flame Based Fluidized Bed Reactor for Nanomaterial Production. U.S. provisional patent application no 623754142016 .

78. Peters, M. S.; Timmerhaus, K. D., Plant Design and Economics for Chemical Engineers. 4th ed.; 1991; p 910.

79. Stanislaus, A.; Marafi, A.; Rana, M. S., Recent advances in the science and technology of ultra low sulfur diesel (ULSD) production. Catal. Today 2010, 153 (1-2), $1-68$.

80. Fan, L.-S.; Zhu, C.; Fan, L.-S.; Zhu., C., Size and Properties of Particles Principles of Gas-Solid Flows. Cambridge University Press: 1998.

81. Brown, H. S. F. a. L. F. In Reactor, ACS Symposium Series, 1981; p 31.

82. Yamabi, S.; Imai, H., Growth conditions for wurtzite zinc oxide films in aqueous solutions. Journal of Materials Chemistry 2002, 12 (12), 3773-3778.

83. NIST Chemistry WebBook.

84. (a) Wegner, K.; Pratsinis, S. E., Scale-up of nanoparticle synthesis in diffusion flame reactors. Chemical Engineering Science 2003, 58 (20), 4581-4589; (b) Wegner, K.; 
Pratsinis, S. E., Gas-phase synthesis of nanoparticles: scale-up and design of flame reactors. Powder Technology 2005, 150 (2), 117-122.

85. Srivastava, V. C., An evaluation of desulfurization technologies for sulfur removal from liquid fuels. Rsc Advances 2012, 2 (3), 759-783.

86. Alsolami, B.; Carneiro, J. T.; Moulijn, J. A.; Makkee, M., On-site low-pressure diesel HDS for fuel cell applications: Deepening the sulfur content to $<=1 \mathrm{ppm}$. Fuel 2011, 90 (10), 3021-3027.

87. Agency, E. P., Control of Air Pollution From Motor Vehicles: Tier 3 Motor Vehicle

Emission and Fuel Standards; Proposed Rule. Federal Register 2013, 78 (98).

88. Inc., W. R., Refinery Catalysts Market Shares, Strategy, and Forecasts, Worldwide,

2012 to 2018.2012.

89. Song, C. S., An overview of new approaches to deep desulfurization for ultraclean gasoline, diesel fuel and jet fuel. Catal. Today 2003, 86 (1-4), 211-263.

90. (a) Ho, T. C., Deep HDS of diesel fuel: chemistry and catalysis. Catal. Today 2004, 98 (1-2), 3-18; (b) Yumoto, M.; Usui, K.; Watanabe, K.; Idei, K.; Yamazaki, H., Development of a cosmo deep HDS catalyst for diesel fuel. Catal. Today 1997, 35 (1-2), 45-50; (c) Ali, S. A.; Ahmed, S.; Ahmed, K. W.; Al-Saleh, M. A., Simultaneous hydrodesulfurization of dibenzothiophene and substituted dibenzothiophenes over phosphorus modified CoMo/A12O3 catalysts. Fuel Process. Technol. 2012, 98, 39-44; (d) Gao, Q.; Ofosu, T. N. K.; Ma, S. G.; Komvokis, V. G.; Williams, C. T.; Segawa, K., Catalyst development for ultra-deep hydrodesulfurization (HDS) of dibenzothiophenes. I: 
Effects of Ni promotion in molybdenum-based catalysts. Catal. Today 2011, 164 (1), $538-543$.

91. (a) Pacheco, M. E.; Salim, V. M. M.; Pinto, J. C., Accelerated Deactivation of Hydrotreating Catalysts by Coke Deposition. Industrial \& Engineering Chemistry Research 2011, 50 (10), 5975-5981; (b) Furimsky, E.; Massoth, F. E., Deactivation of hydroprocessing catalysts. Catal. Today 1999, 52 (4), 381-495.

92. Bartholomew, C. H., Mechanisms of catalyst deactivation. Applied Catalysis aGeneral 2001, 212 (1-2), 17-60.

93. (a) Babich, I. V.; Moulijn, J. A., Science and technology of novel processes for deep desulfurization of oil refinery streams: A review. Fuel 2003, 82 (6), 607-631; (b) Tawara, K.; Nishimura, T.; Iwanami, H.; Nishimoto, T.; Hasuike, T., New hydrodesulfurization catalyst for petroleum-fed fuel cell vehicles and cogenerations. Industrial \& Engineering Chemistry Research 2001, 40 (10), 2367-2370; (c) Zhang, J.; Liu, Y.; Tian, S.; Chai, Y.; Liu, C., Reactive adsorption of thiophene on $\mathrm{Ni} / \mathrm{ZnO}$ adsorbent: Effect of $\mathrm{ZnO}$ textural structure on the desulfurization activity. Journal of Natural Gas Chemistry 2010, 19 (3), 327-332; (d) Petzold, F. G.; Jasinski, J.; Clark, E. L.; Kim, J. H.; Absher, J.; Toufar, H.; Sunkara, M. K., Nickel supported on zinc oxide nanowires as advanced hydrodesulfurization catalysts. Catal. Today 2012, 198 (1), 219227; (e) Sharma, M.; Vyas, R. K.; Singh, K., A review on reactive adsorption for potential environmental applications. Adsorpt.-J. Int. Adsorpt. Soc. 2013, 19 (1), 161188.

94. (a) Da Costa-Serra, J. F.; Guil-Lopez, R.; Chica, A., Co/ZnO and Ni/ZnO catalysts for hydrogen production by bioethanol steam reforming. Influence of $\mathrm{ZnO}$ 
support morphology on the catalytic properties of $\mathrm{Co}$ and $\mathrm{Ni}$ active phases. Int. J. Hydrog. Energy 2010, 35 (13), 6709-6716; (b) Behl, M.; Yeom, J.; Lineberry, Q.; Jain, P. K.; Shannon, M. A., A regenerable oxide-based H2S adsorbent with nanofibrous morphology. Nat. Nanotechnol. 2012, 7 (12), 810-815.

95. Zhang, Y.; Yang, Y.; Han, H.; Yang, M.; Wang, L.; Zhang, Y.; Jiang, Z.; Li, C., Ultra-deep desulfurization via reactive adsorption on $\mathrm{Ni} / \mathrm{ZnO}$ : The effect of $\mathrm{ZnO}$ particle size on the adsorption performance. Applied Catalysis, B: Environmental 2012, 119-120, 13-19.

96. Xiong, Q.; Chen, G.; Acord, J.; Liu, X.; Zengel, J.; Gutierrez, H.; Redwing, J.; Lew Yan Voon, L.; Lassen, B.; Eklund, P., Optical properties of rectangular crosssectional ZnS nanowires. Nano letters 2004, 4 (9), 1663-1668.

97. Zhao, C.; Chen, A.; Ji, X.; Zhu, Y.; Gui, X.; Huang, F.; Tang, Z., Growth of Vertically aligned $\mathrm{ZnO}$ nanowire arrays on $\mathrm{ZnO}$ single crystals. Materials Letters 2015.

98. Fujikawa, T., Development of new CoMoHDS catalyst for ultra-low sulfur diesel fuel production. J. Jpn. Pet. Inst 2007, 50 (5), 249-261.

99. Hui, H.; Tan, O. K.; Lee, Y. C.; Tse, M. S.; Guo, J.; White, T., Effects of plasma treatment on the growth of $\mathrm{SnO} 2$ nanorods from $\mathrm{SnO} 2$ thin films. Nanotechnology 2006, $17(3), 743$.

100. Chandra, D.; Mukherjee, N.; Mondal, A.; Bhaumik, A., Design and Synthesis of Nanostructured Porous SnO2 with High Surface Areas and Their Optical and Dielectric Properties. The Journal of Physical Chemistry C 2008, 112 (23), 8668-8674. 
101. Dai, Z. R.; Gole, J. L.; Stout, J. D.; Wang, Z. L., Tin Oxide Nanowires, Nanoribbons, and Nanotubes. The Journal of Physical Chemistry B 2002, 106 (6), 12741279.

102. Liu, Z.; Zhang, D.; Han, S.; Li, C.; Tang, T.; Jin, W.; Liu, X.; Lei, B.; Zhou, C., Laser Ablation Synthesis and Electron Transport Studies of Tin Oxide Nanowires. Advanced Materials 2003, 15 (20), 1754-1757.

103. Zhang, H.; Tan, Z.; Xu, P.; Oh, K.; Wu, R.; Shi, W.; Jiao, Z., Preparation of $\mathrm{SnO}<\mathrm{sub}>2</$ sub $>$ Nanowires by Solvent-Free Method Using Mesoporous Silica Template and Their Gas Sensitive Properties. Journal of Nanoscience and Nanotechnology 2011, 11 (12), 11114-11118.

104. Wang, Y.; Jiang, X.; Xia, Y., A Solution-Phase, Precursor Route to Polycrystalline SnO2 Nanowires That Can Be Used for Gas Sensing under Ambient Conditions. Journal of the American Chemical Society 2003, 125 (52), 16176-16177.

105. Zhang, D.-F.; Sun, L.-D.; Xu, G.; Yan, C.-H., Size-controllable one-dimensinal SnO2 nanocrystals: synthesis, growth mechanism, and gas sensing property. Physical Chemistry Chemical Physics 2006, 8 (42), 4874-4880.

106. (a) Cheng, B.; Russell, J. M.; Shi; Zhang, L.; Samulski, E. T., Large-Scale, Solution-Phase Growth of Single-Crystalline SnO2 Nanorods. Journal of the American Chemical Society 2004, 126 (19), 5972-5973; (b) Jian, J. K.; Chen, X. L.; Wang, W. J.; Dai, L.; Xu, Y. P., Growth and morphologies of large-scale $\mathrm{SnO} 2$ nanowires, nanobelts and nanodendrites. Appl Phys A 2003, 76 (2), 291-294.

107. (a) Cabanas, A.; Darr, J. A.; Lester, E.; Poliakoff, M., A continuous and clean one-step synthesis of nano-particulate $\mathrm{CeZrO}$ solid solutions in near-critical water. 
Chemical Communications 2000, (11), 901-902; (b) Chung, C.-C.; Chung, T.-W.; Yang, T. C. K., Rapid Synthesis of Titania Nanowires by Microwave-Assisted Hydrothermal Treatments. Industrial \& Engineering Chemistry Research 2008, 47 (7), 2301-2307.

108. Zhou, J. X.; Zhang, M. S.; Hong, J. M.; Yin, Z., Raman spectroscopic and photoluminescence study of single-crystalline $\mathrm{SnO} 2$ nanowires. Solid State Communications 2006, 138 (5), 242-246.

109. Wang, Y.; Lee, J. Y.; Deivaraj, T. C., Controlled Synthesis of V-shaped SnO2 Nanorods. The Journal of Physical Chemistry B 2004, 108 (36), 13589-13593.

110. Beltrán, A.; Andrés, J.; Longo, E.; Leite, E. R., Thermodynamic argument about SnO2 nanoribbon growth. Applied Physics Letters 2003, 83 (4), 635-637.

111. Ostrikov, K.; Neyts, E. C.; Meyyappan, M., Plasma nanoscience: from nanosolids in plasmas to nano-plasmas in solids. Advances in Physics 2013, 62 (2), 113-224.

112. Qin, L.; Xu, J.; Dong, X.; Pan, Q.; Cheng, Z.; Xiang, Q.; Li, F., The template-free synthesis of square-shaped $\mathrm{SnO} 2$ nanowires: the temperature effect and acetone gas sensors. Nanotechnology 2008, 19 (18), 185705.

113. (a) Davide, M.; Sankaran, M., Microplasmas for nanomaterials synthesis. Journal of Physics D: Applied Physics 2010, 43 (32), 323001; (b) Mozetič, M.; Cvelbar, U.; Sunkara, M. K.; Vaddiraju, S., A Method for the Rapid Synthesis of Large Quantities of Metal Oxide Nanowires at Low Temperatures. Advanced Materials 2005, 17 (17), 21382142.

114. Martinez, C. J.; Hockey, B.; Montgomery, C. B.; Semancik, S., Porous Tin Oxide Nanostructured Microspheres for Sensor Applications. Langmuir 2005, 21 (17), 79377944. 
115. (a) Derrien, G.; Hassoun, J.; Panero, S.; Scrosati, B., Nanostructured Sn-C Composite as an Advanced Anode Material in High-Performance Lithium-Ion Batteries. Advanced Materials 2007, 19 (17), 2336-2340; (b) Winter, M.; Besenhard, J. O., Electrochemical lithiation of tin and tin-based intermetallics and composites. Electrochimica Acta 1999, 45 (1-2), 31-50.

116. Wachtler, M.; Winter, M.; Besenhard, J. O., Anodic materials for rechargeable Libatteries. Journal of Power Sources 2002, 105 (2), 151-160.

117. Mei, L.; Li, C.; Qu, B.; Zhang, M.; Xu, C.; Lei, D.; Chen, Y.; Xu, Z.; Chen, L.; Li, Q.; Wang, T., Small quantities of cobalt deposited on tin oxide as anode material to improve performance of lithium-ion batteries. Nanoscale 2012, 4 (18), 5731-5737.

118. Nguyen, T. Q.; Thapa, A. K.; Vendra, V. K.; Jasinski, J. B.; Sumanasekera, G. U.; Sunkara, M. K., High rate capacity retention of binder-free, tin oxide nanowire arrays using thin titania and alumina coatings. RSC Advances 2014, 4 (7), 3312-3317.

119. Montenegro, D. N.; Hortelano, V.; Martínez, O.; Martínez-Tomas, M. C.; Sallet, V.; Muñoz-Sanjosé, V.; Jiménez, J., Non-radiative recombination centres in catalyst-free $\mathrm{ZnO}$ nanorods grown by atmospheric-metal organic chemical vapour deposition. Journal of Physics D: Applied Physics 2013, 46 (23), 235302.

120. Boukamp, B. A.; Lesh, G. C.; Huggins, R. A., All-Solid Lithium Electrodes with Mixed-Conductor Matrix. Journal of The Electrochemical Society 1981, 128 (4), 725729.

121. Li, N.; Martin, C. R.; Scrosati, B., A High-Rate, High-Capacity, Nanostructured Tin Oxide Electrode. Electrochemical and Solid-State Letters 2000, 3 (7), 316-318. 
122. Bridel, J.-S.; Grugeon, S.; Laruelle, S.; Hassoun, J.; Reale, P.; Scrosati, B.; Tarascon, J.-M., Decomposition of ethylene carbonate on electrodeposited metal thin film anode. Journal of Power Sources 2010, 195 (7), 2036-2043.

123. Ding, X.-L.; Sun, Q.; Lu, F.; Fu, Z.-W., Nanocomposite SnO2-Se thin film as anode material for lithium-ion batteries. Journal of Power Sources 2012, 216 (0), 117123.

124. Yu, Y.; Chen, C.-H.; Shi, Y., A Tin-Based Amorphous Oxide Composite with a Porous, Spherical, Multideck-Cage Morphology as a Highly Reversible Anode Material for Lithium-Ion Batteries. Advanced Materials 2009, 21 (35), n/a-n/a.

125. Xia, W.; Wang, Y.; Luo, Y.; Li, J.; Fang, Y.; Gu, L.; Peng, J.; Sha, J., Facile approach to synthesize $\mathrm{SnO} 2$ nanoparticles@carbon nanofibers as anode materials for lithium-ion battery. Journal of Power Sources 2012, 217 (0), 351-357.

126. Park, M.-S.; Wang, G.-X.; Kang, Y.-M.; Wexler, D.; Dou, S.-X.; Liu, H.-K., Preparation and Electrochemical Properties of $\mathrm{SnO} 2$ Nanowires for Application in Lithium-Ion Batteries. Angewandte Chemie 2007, 119 (5), 764-767.

127. Kim, D.-W.; Hwang, I.-S.; Kwon, S. J.; Kang, H.-Y.; Park, K.-S.; Choi, Y.-J.; Choi, K.-J.; Park, J.-G., Highly Conductive Coaxial SnO2-In2O3 Heterostructured Nanowires for Li Ion Battery Electrodes. Nano Letters 2007, 7 (10), 3041-3045.

128. Ko, Y.-D.; Kang, J.-G.; Park, J.-G.; Lee, S.; Kim, D.-W., Self-supported SnO 2 nanowire electrodes for high-power lithium-ion batteries. Nanotechnology 2009, 20 (45), 455701.

129. Zhang, L. Q.; Liu, X. H.; Liu, Y.; Huang, S.; Zhu, T.; Gui, L.; Mao, S. X.; Ye, Z. Z.; Wang, C. M.; Sullivan, J. P.; Huang, J. Y., Controlling the Lithiation-Induced Strain 
and Charging Rate in Nanowire Electrodes by Coating. ACS Nano 2011, 5 (6), 48004809.

130. Ji, G.; Ma, Y.; Ding, B.; Lee, J. Y., Improving the Performance of High Capacity Li-Ion Anode Materials by Lithium Titanate Surface Coating. Chemistry of Materials 2012, 24 (17), 3329-3334.

131. Park, H.; Song, T.; Han, H.; Devadoss, A.; Yuh, J.; Choi, C.; Paik, U., SnO2 encapsulated $\mathrm{TiO} 2$ hollow nanofibers as anode material for lithium ion batteries. Electrochemistry Communications 2012, 22 (0), 81-84.

132. Wu, X.; Zhang, S.; Wang, L.; Du, Z.; Fang, H.; Ling, Y.; Huang, Z., Coaxial SnO2@TiO2 nanotube hybrids: from robust assembly strategies to potential application in Li+ storage. Journal of Materials Chemistry 2012, 22 (22), 11151-11158.

133. Jung, Y. S.; Cavanagh, A. S.; Riley, L. A.; Kang, S.-H.; Dillon, A. C.; Groner, M. D.; George, S. M.; Lee, S.-H., Ultrathin Direct Atomic Layer Deposition on Composite Electrodes for Highly Durable and Safe Li-Ion Batteries. Advanced Materials 2010, 22 (19), 2172-2176.

134. Meduri, P.; Clark, E.; Dayalan, E.; Sumanasekera, G. U.; Sunkara, M. K., Kinetically limited de-lithiation behavior of nanoscale tin-covered tin oxide nanowires. Energy \& Environmental Science 2011, 4 (5), 1695-1699.

135. Demir-Cakan, R.; Hu, Y.-S.; Antonietti, M.; Maier, J.; Titirici, M.-M., Facile One-Pot Synthesis of Mesoporous SnO2 Microspheres via Nanoparticles Assembly and Lithium Storage Properties. Chemistry of Materials 2008, 20 (4), 1227-1229. 
136. Aurbach, D.; Nimberger, A.; Markovsky, B.; Levi, E.; Sominski, E.; Gedanken, A., Nanoparticles of $\mathrm{SnO}$ Produced by Sonochemistry as Anode Materials for Rechargeable Lithium Batteries. Chemistry of Materials 2002, 14 (10), 4155-4163.

137. Li, N.; Martin, C. R., A High-Rate, High-Capacity, Nanostructured Sn-Based Anode Prepared Using Sol-Gel Template Synthesis. Journal of The Electrochemical Society 2001, 148 (2), A164-A170.

138. Courtney, I. A.; Dahn, J. R., Electrochemical and In Situ X-Ray Diffraction Studies of the Reaction of Lithium with Tin Oxide Composites. Journal of The Electrochemical Society 1997, 144 (6), 2045-2052.

139. (a) Yildirim, H.; Greeley, J.; Sankaranarayanan, S. K. R. S., Effect of Concentration on the Energetics and Dynamics of Li Ion Transport in Anatase and Amorphous TiO2. The Journal of Physical Chemistry C 2011, 115 (31), 15661-15673; (b) Fang, H.-T.; Liu, M.; Wang, D.-W.; Sun, T.; Guan, D.-S.; Li, F.; Zhou, J.; Sham, T.K.; Cheng, H.-M., Comparison of the rate capability of nanostructured amorphous and anatase TiO 2 for lithium insertion using anodic TiO 2 nanotube arrays. Nanotechnology 2009, 20 (22), 225701.

140. Glass, A. M.; Nassau, K., Lithium ion conduction in rapidly quenched Li[sub 2]O-Al[sub 2] [sub 3], Li[sub 2]O-Ga[sub 2] [sub 3], and Li[sub 2]O-Bi[sub 2]O[sub 3] glasses. Journal of Applied Physics 1980, 51 (7), 3756-3761.

141. Liu, Y.; Hudak, N. S.; Huber, D. L.; Limmer, S. J.; Sullivan, J. P.; Huang, J. Y., In Situ Transmission Electron Microscopy Observation of Pulverization of Aluminum Nanowires and Evolution of the Thin Surface Al2O3 Layers during LithiationDelithiation Cycles. Nano Letters 2011, 11 (10), 4188-4194. 
142. Cao, F.-F.; Deng, J.-W.; Xin, S.; Ji, H.-X.; Schmidt, O. G.; Wan, L.-J.; Guo, Y.G., Cu-Si Nanocable Arrays as High-Rate Anode Materials for Lithium-Ion Batteries. Advanced Materials 2011, 23 (38), 4415-4420.

143. Nishikiori, H.; Setiawan, R. A.; Miyamoto, K.; Sukmono, G.; Uesugi, Y.; Teshima, K.; Fujii, T., Photoinduced electron transport in dye-containing titania gel films. RSC Advances 2012, 2 (10), 4258-4267.

144. Cheng, H.-M.; Wang, F.-M.; Chu, J. P.; Santhanam, R.; Rick, J.; Lo, S.-C., Enhanced Cycleabity in Lithium Ion Batteries: Resulting from Atomic Layer Depostion of $\mathrm{A} 12 \mathrm{O} 3$ or $\mathrm{TiO} 2$ on LiCoO2 Electrodes. The Journal of Physical Chemistry C 2012, $116(14), 7629-7637$.

145. Shokuhfar, T.; Arumugam, G. K.; Heiden, P. A.; Yassar, R. S.; Friedrich, C., Direct Compressive Measurements of Individual Titanium Dioxide Nanotubes. ACS Nano 2009, 3 (10), 3098-3102.

146. Reich, S.; Thomsen, C.; Ordejón, P., Elastic properties of carbon nanotubes under hydrostatic pressure. Physical Review B 2002, 65 (15), 153407.

147. Huang, J. Y.; Zhong, L.; Wang, C. M.; Sullivan, J. P.; Xu, W.; Zhang, L. Q.; Mao, S. X.; Hudak, N. S.; Liu, X. H.; Subramanian, A.; Fan, H.; Qi, L.; Kushima, A.; Li, J., In Situ Observation of the Electrochemical Lithiation of a Single SnO2 Nanowire Electrode. Science 2010, 330 (6010), 1515-1520.

148. Wang, C.-M.; Xu, W.; Liu, J.; Zhang, J.-G.; Saraf, L. V.; Arey, B. W.; Choi, D.; Yang, Z.-G.; Xiao, J.; Thevuthasan, S.; Baer, D. R., In Situ Transmission Electron Microscopy Observation of Microstructure and Phase Evolution in a SnO2 Nanowire during Lithium Intercalation. Nano Letters 2011, 11 (5), 1874-1880. 
149. Zhang, L. Q.; Liu, X. H.; Perng, Y.-C.; Cho, J.; Chang, J. P.; Mao, S. X.; Ye, Z. Z.; Huang, J. Y., Direct observation of Sn crystal growth during the lithiation and delithiation processes of SnO2 nanowires. Micron 2012, 43 (11), 1127-1133.

150. Tu, K. N., Irreversible processes of spontaneous whisker growth in bimetallic $\mathrm{Cu}-$ Sn thin-film reactions. Physical Review B 1994, 49 (3), 2030-2034.

151. Fan, H. J.; Gösele, U.; Zacharias, M., Formation of Nanotubes and Hollow Nanoparticles Based on Kirkendall and Diffusion Processes: A Review. Small 2007, 3 (10), 1660-1671. 


\title{
CURRICULUM VITAE
}

\author{
TU NGUYEN
}

Department of Chemical Engineering and Conn Center for Renewable Energy Research University of Louisville, KY 40292

Phone: (502) 526-2607, Email: tutu.nwin@gmail.com

\section{EDUCATION}

Ph.D., Chemical Engineering (GPA: 3.970/4.0)

August 2016

University of Louisville (UofL)

Louisville, KY

- Thesis: Scalable production and applications of metal oxide nanowires

M.S., Chemical Engineering

Kongju National University (KNU)

- Thesis: Vapor-phase synthesis of alumina nanoparticles through thermal decomposition of aluminum tri-isopropoxide

B.E., Chemical Engineering (Honor Program)

Hanoi University Science and Technology (HUST)

- Thesis: Wet Chemical Synthesis of Alkali Silicates
July 2007

Hanoi, Vietnam

\section{WORK EXPERIENCE}

Process Engineer

Advanced Energy Materials, LLC
September 2016 - present

Louisville, KY

- Designed, assembled, and optimized a new fluidized bed reactor based hydrocarbon flame for bulk production of zinc oxide nanowires

- Scaled-up the production of nanowires to yield tons-quantities

Graduate Intern

October 2014 - August 2016

Advanced Energy Materials, LLC

Louisville, KY

- Designed, assembled, and optimized a new fluidized bed reactor based on atmospheric microwave plasma flame and hydrocarbon flame for bulk production of zinc oxide nanowires

- Scaled-up the production of nanowires to yield kilogram-quantities

Research Assistant/Teaching Assistant

Department of Chemical Engineering, UofL
August 2011 - September 2014 Louisville, KY

- 4+ years hands-on experience in assembly, troubleshooting and optimization of plasma reactors 
- Developed new plasma technique called "Solvo-Plasma" to produce bulk quantities of tin oxide nanowires, potassium stannate nanowires

- Synthesized tin oxide nanowires using chemical vapor deposition and developed a surface modification procedure using atomic layer deposition for achieving high capacity retention of tin oxide nanowires in lithium ion batteries

- Performed process modification and a reactor design modifications of atmospheric microwave plasma reactor that resulted high yields of zinc oxide nanowires

- Developed standard operating procedures for custom-made atmospheric microwave plasma reactor

- Served as a teaching assistant for CHE620 (Transport Phenomena), CHE694 (Materials Characterization)

\section{Research Assistant}

Department of Mechanical Engineering, FSU

August 2010 - April 2011

Tallahassee, FL

- Localized synthesis of silicon and germanium nanowires on resistivity heated MEMS devices using chemical vapor deposition

- Developed a model for temperature distribution surrounding a MEMS microbridge using COMSOL

\section{Research Assistant}

Department of Chemical Engineering, KNU

2008 - June 2010

Cheonan, South Korea

- Synthesized alumina and silica nanoparticles using aluminum trichloride or aluminum tri-isopropoxide and silicon tetrachloride in gas phase, respectively

\section{PATENTS}

1) Sunkara M.K., Nguyen T. Q., Guhy L.H., Paxton W. F., Flame Based Fluidized Bed Reactor for Nanomaterial Production. (U.S. Provisional Application no 62/375,414)

\section{PUBLICATIONS}

12) Nguyen T. Q., Guhy L.H., Paxton W.F., Jasinki J.B., and Sunkara M.K., Flame based production of zinc oxide nanorods (manuscript in preparation 2016)

11) Kumar B., Atla V., Brian J. P., Kumari S., Nguyen T. Q., Sunkara M.K., Spurgeon J.M., Reduced $\mathrm{SnO}_{2}$ porous nanowires with high-density grain boundaries as catalysts for efficient electrochemical $\mathrm{CO}_{2}$-to-HCOOH (submitted 2016)

10) Nguyen T.Q.*, Kumar V.*, Clark E.L., Jasinksi J.B., Sunkara M. K., A New Solvoplasma Oxidation Method for Scalable Manufacturing of Metal Oxide Nanowires (submitted 2016) *contributed equally

9) Nambo A., He J., Nguyen T. Q., Druffel T., and Sunkara M.K., Ultrafast Carbon Dioxide Sorption Kinetics Using Lithium Silicate Nanowires (submitted 2016)

8) Nguyen, T. Q.; Atla, V.; Vendra, V. K.; Thapa, A. K.; Jasinski, J. B.; Druffel, T. L.; Sunkara, M. K. Scalable solvo-plasma production of porous tin oxide nanowires. Chemical Engineering Science 2016, 154, 20-26.

7) Gupta, M.; He, J.; Nguyen, T. Q.; Petzold, F.; Fonseca, D.; Jasinski, J. B.; Sunkara, M. K. "Nanowire catalysts for ultra-deep hydro-desulfurization and aromatic hydrogenation". Applied Catalysis B: Environmental 2016, 180, 246-254. 
6) Martinez-Garcia, A.; Thapa, A. K.; Dharmadasa, R.; Nguyen, T. Q.; Jasinski, J.; Druffel, T. L.; Sunkara, M. K. High rate and durable, binder free anode based on silicon loaded $\mathrm{MoO}_{3}$ nanoplatelets. Scientific Reports 2015, 5, 10530.

5) Vendra, V. K.; Nguyen, T. Q.; Thapa, A. K.; Jasinski, J. B.; Sunkara, M. K. Scalable synthesis and surface stabilization of $\mathrm{Li}_{2} \mathrm{MnO}_{3} \mathrm{NWs}$ as high rate cathode materials for Li-ion batteries. RSC Advances 2015, 5, 36906-36912.

4) Nguyen, T. Q.; Thapa, A. K.; Vendra, V. K.; Jasinski, J. B.; Sumanasekera, G. U.; Sunkara, M. K. High rate capacity retention of binder-free, tin oxide nanowire arrays using thin titania and alumina coatings. RSC Advances 2014, 4, 3312-3317.

3) Vendra, V. K.; Nguyen, T. Q.; Druffel, T.; Jasinski, J. B.; Amos, D. A.; Sunkara, M. K. Nanowire architectures for iodide free dye-sensitized solar cells. Journal of Materials Chemistry A 2014, 2, 3543-3550.

2) Jayasinghe, R.; Thapa, A. K.; Dharmasena, R. R.; Nguyen, T. Q.; Pradhan, B. K.; Paudel, H. S.; Jasinski, J. B.; Sherehiy, A.; Yoshio, M.; Sumanasekera, G. U. Optimization of MultiWalled Carbon Nanotube based CFx electrodes for improved primary and secondary battery performances. Journal of Power Sources 2014, 253, 404-411.

1) Nguyen, T. Q.; Park, K. Y.; Jung, K. Y.; Cho, S. B. Vapor-phase synthesis of a solid precursor for $\alpha$-alumina through a catalytic decomposition of aluminum triisopropoxide. Materials Research Bulletin 2011, 46, 2199-2203.

\section{PRESENTATIONS}

10) T. Q. Nguyen, V. K. Vendra, J. B. Jasinski, and M. K. Sunkara. Scalable Production of Nanowire Based Materials Using a Solvo-Plasma Technique. RE3 Workshop, UofL, March 22-24, 2015. Louisville, KY

9) T. Q. Nguyen, A. K. Thapa, V. K. Vendra, J. B. Jasinski, and M. K. Sunkara. Tin Oxide Nanowires as High Energy Density Anode for Lithium-ion Batteries. 225rd Annual ECS meeting, May 11-15, 2014, Orlando, FL

8) T. Q. Nguyen, A. K. Thapa, V. K. Vendra, J. B. Jasinski, G. U. Sumanasekera and M. K. Sunkara. Tin Oxide Nanowires as High Energy Density Anode for Lithium-ion Batteries. ECI Conference June 23-26, 2013. Newport Beach, CA

7) T. Q. Nguyen, A. K. Thapa, V. K. Vendra, J. B. Jasinski, G. U. Sumanasekera and M. K. Sunkara. Scalable Synthesis of Tin Oxide Nanowires for Lithium-ion Battery Anodes. 23rd North American Catalysis Society Meeting, June 2-7, 2013. Louisville, KY

6) T. Q. Nguyen, and M. K. Sunkara. Scalable Production of Nanowire Arrays and Powders Using Plasmas. 223rd Annual ECS meeting, May 12-16, 2013, Toronto, ON

5) T. Q. Nguyen, A. K. Thapa, V. K. Vendra, J. B. Jasinski, G. U. Sumanasekera and M. K. Sunkara. Enhanced Electrochemical Performance of Tin Oxide Nanowire Anodes using Atomic Layer Deposition of Titania or Alumina. E-expo, UofL, March 24-26, 2013. Louisville, KY

4) T. Q. Nguyen, A. K. Thapa, V. K. Vendra, J. B. Jasinski, G. U. Sumanasekera and M. K. Sunkara. Tin-based Oxide Nanowires as Anodes for Lithium-ion Batteries", 5th Annual Nanotechnology and Nanomedicine Symposium, Sept. 21-22, 2012. Sullivan University, Louisville, KY 
3) T. Q. Nguyen, H. K. Park and K. Y. Park. Vapor-phase synthesis of alumina particles through thermal decomposition of aluminum tri-isopropoxide. Korean Institute of Chemical Engineers (KIChE), Fall Meeting Oct. 21-23, 2009, Seoul, Korea

2) T. Q. Nguyen, Y. S. Yoo and K. Y. Park. Preparation of spherical alumina by thermal decomposition of aluminum tri-isopropoxide vapor with hydrogen chloride as catalyst. Proceedings The 9th Korean Conference on Aerosol and Particle Technology, July 02-04, 2009. Youngpyong, Korea

1) T. Q. Nguyen, S. Y. Kim and K. Y. Park. Synthesis of silica particles by vapor-phase hydrolysis of $\mathrm{SiCl}_{4}$. Korean Institute of Chemical Engineers (KIChE), Fall Meeting Oct. 2224, 2008. Busan, Korea

\section{TECHNICAL SKILLS}

- Reactor: Atmospheric Microwave and RF Plasma, Chemical Vapor Deposition, Atomic Layer Deposition, Fluidized Bed Reactor

- Characterization: Scanning Electron Microscopy, Transmission Electron Microscopy, X-ray Diffraction, Energy-dispersive X-ray spectroscopy, Cyclic Voltametry, Raman, Fourier Transform Infrared Spectroscopy, Thermogravimetric Analysis, Gas Chromatography

- Other: Digital Micrograph, Solidworks, MS Offices, Origin, Illustrator, Photoshop

\section{AWARDS}

- 2016 Graduate Dean's citation for Outstanding PhD Dissertation

- Travel grant, Engineering Conferences International: Massive Energy Storage for the Broader Use of Renewable Energy Sources, June 2013

- Graduate Fellowship, The University of Louisville, 2011-2013

- Two-time Bronze Medal, The National Olympiad in Applied Chemistry for Undergraduate Students, Vietnamese Ministry of Education and Training and Vietnamese Chemical Society, 2005 and 2006

- $3^{\text {rd }}$ Prize, The National Chemistry Olympiad for Excellent Students of Grade 12, 2001

- Two-time $1^{\text {st }}$ Prize, Annual Chemistry Olympiad, State of Bac Ninh, 2001 and 2002

\section{MEMBERSHIPS}

- Electrochemical Society

- Korean Institute of Chemical Engineer 